MORPHOLOGY OF

WATER-BLOWN FLEXIBLE POLYURETHANE FOAMS,

by

James Paul Armistead,

Thesis submitted to the Faculty of the

Virginia Polytechnic Institute and State University

in partial fulfillment of the requirements for the degree of

MASTER OF SCIENCE

in

Chemical Engineering

APPROVED:

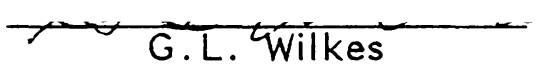

TF. Sebba W/J. Glassen

November, 1985

Blacksburg, Virginia 


\section{MORPHOLOGY OF \\ WATER-BLOWN FLEXIBLE POLYURETHANE FOAMS \\ by}

James Paul Armistead

Committee Chairman: Garth L. Wilkes

Chemical Engineering

(ABSTRACT)

A series of four water-blown flexible polyurethane foams was produced in which the water content was varied from 2 to $5 \mathrm{pph}$ at a constant isocyanate index of 110. A portion of each foam was thermally compression molded into a plaque. The morphology of the foams and plaques was investigated using DMS, DSC, FTIR, TEM, SEM, swelling, WAXS, and SAXS. A high degree of phase separation occurs in these foams and the degree of phase separation is independent of water (hard segment) content. In the foam with the lowest water content the morphology is similar to that of typical segmented urethane elastomers. Small hard segment domains are present with a correlation distance of roughly 7.0 nanometers. When the water content is increased a binodal distribution of hard segments appears. There are the small hard segment domains typical of segmented urethane elastomers as well as large hard segment aggregates greater than 100 nanometers in diameter. The large domains are thought to be aggregates of polyurea that precipitated during the manufacture of the foam. The foam making process successfully incorporated the trifunctional polyols into a network indicating a high degree of polymerization for the hydroxyl-isocyanate 
reaction. Unreacted isocyanate is present in the foams a month after curing. It is believed to be trapped in the large urea aggregates. WAXS patterns of the foams suggest hard segment ordering that may be of a paracrystalline nature but certainly lacking in true crystallinity. 


\section{ACKNOWLEDGEMENTS}

I would like to thank my major professor $\mathrm{Dr}$. Wilkes for his patience, concern, and support, without which I probably would not have remained in Blacksburg to complete this work.

I would also like to thank Dr. Glasser and Dr. Sebba for the interest they showed in my work and the time and effort they spent reviewing my thesis.

I would like to thank Rick Allen and Dr. Lin for their assistance in using the ORNL facilities and Dinesh Tyagi and Brandt Carter for many helpful discussions.

Finally, I would like to thank all of the group members for their everyday greetings, smiles, and conversion. In particular I would like to thank Dinesh and Ruby, Brandt, Marty and Martha, and Bruce. 


\section{TABLE OF CONTENTS}

CHAPTER ONE: INTRODUCTION AND LITERATURE REVIEW

1.1 Introduction

1.2 Chemistry

1.3 Formulation Components

1.4 Foam Manufacture 22

1.5 Recent Work 25

1.6 Summary 31

CHAPTER TWO: SMALL ANGLE SCATTERING THEORY

2.1 Introduction 33

2.2 SAXS Theory 39

2.2.1 Scattering Amplitude 40

2.2.2 Particle Size 46

2.2.3 Babinet Principle 48

2.2.4 Correlation Function 49

2.2.5 Porod's Law 51

2.2.6 Degree of Phase Separation 54

2.3 Summary $\quad 57$

CHAPTER THREE: EXPERIMENTAL

3.1 Foam Preparation 59

3.2 Methods of Analysis 61

CHAPTER FOUR: RESULTS AND DISCUSSION

4.1 Morphological Considerations 73

4.2 Macroscopic Structure $\quad 76$

4.3 Mechanical Properties $\quad 80$

4.4 Thermal Characterization 84

4.5 FTIR 90

4.6 TEM 92

4.7 SEM 95

4.8 Swelling and Extraction 98

4.9 WAXS 100

4.10 SAXS 102

4.11 Summary 125

4.12 Conclusions and Recommendations 130

REFERENCES

TABLES 140

FIGURES $\quad 152$

APPENDICES 207

$\begin{array}{ll}\text { VITA } & 236\end{array}$ 
preface

Flexible polyurethane foams have been produced on a commercial scale in this country for over twentyfive years yet the operation is still, in many ways, an art. One reason for this is that in the production of polyurethane foams, the blowing and gelling reactions occur simultaneously. Any change in chemistry or processing conditions alters both the cell structure and the morphology of the material comprising the cell structure. As a result, there is no way to evaluate the material independent of cell structure or cell structure independent of material. Changes in chemical and processing variables have been extensively studied as to their influence on bulk properties of the foam and polyurethane foams with a wide range of physical properties can be produced $(1-5)$.

This 'technological' approach to the development of polyurethane foams has been very successful and presently, the yearly production of polyurethane foams far outweighs other types of foam plastics. One must think, however, that a more fundamental understanding of the foaming kinetics and the structure/property relationships of the resultant foam would be an aid to the development of new and better foams. With this in mind, a project was undertaken with the Dow Chemical Company. The workers at Dow prepared a basic series of slabstock foams varying the water content or the tin catalyst concentration while maintaining a constant isocyanate index. A section 
of each foam was thermally compression molded into a solid plaque. Analyses were performed on both the foams and the corresponding plaques. Dow workers used FTIR spectroscopy to study the composition of the foams, DMS to study the elastic properties, DSC to look for evidence of phase separation, and TEM to access the size and distribution of phase separated regions. The major thrust of the work in this laboratory was characterization of the phase separated domains with small angle $x$-ray scattering (SAXS). WAXS, SEM, and swelling were also used to study the foams. This thesis is a summary of the overall investigation with emphasis on the authors work. 


\section{CHAPTER ONE: INTRODUCTION AND LITERATURE REVIEW}

\subsection{INTRODUCTION}

The term 'polyurethane' is used to describe a large class of polymers that contain recurring urethane (-NH-CO-O-) linkages. In most commercially important solid or foam polyurethanes, the urethane groups are generated to link oligomers together and are not the predominate groups in the polymer backbone. The properties of the material are often more heavily influenced by the types of oligomer than the presence of urethane groups. Through the use of different oligomers, polyurethanes are produced with a broad range of physical properties. Polyurethanes can be manufactured as adhesives, surface coatings, elastomers, rigid foams, or flexible foams. Flexible polyurethane foams are the focus of this paper.

Present-day flexible polyurethane foams are the benefactors of over thirty years of production experience and technical innovation. A brief look at the history behind polyurethanes and foam production will serve as an introduction to this field.

Synthesis of isocyanates and urethanes was first reported by Wohler in 1828. In 1884, Hentschel developed phosgenation of primary amines as a practical method of synthesizing isocyanates. Not much 
attention was given to these discoveries until the 1930's, when duPont obtained patents for nylon and began commercial production. The similarity between the hydrogen-bonding amide linkage in nylon (-CO$\mathrm{NH}-)$ and a urethane linkage was noticed by Otto Bayer in Germany and duPont in the United States.

In 1939 and 1940, duPont received a series of patents concerning the reactions of polyisocyanates with glycols, polyamides, polyesters, and polyethers. They claimed the products were useful as plastics, films, and fibers but, at the time, did not pursue large-scale commercial production.

Bayer and other workers in Germany obtained a series of German patents for their work with polyurethanes dating from as early as 1937. The early work was concerned with producing fibers and plastics. These products could not compete with nylon but were commercially marketed in Germany. Attention was also given to using polyisocyanates in surface coatings and elastomers. In 1947, Bayer disclosed the discovery of the first rigid polyurethane foam. The first flexible polyurethane foam was reported by Hochlen in 1952 . It was made by reacting hydroxyl-terminated polyesters with diisocyanates in the presence of water. By 1955, polyester-based flexible foams were produced on a commercial scale in both Germany and the United States.

The first flexible polyether-based polyurethane foams were developed in the United States in 1957. The foams were produced in a 
two-step process in which the polyether polyols were capped by reaction with excess diisocyanate in the first step and then reacted with water and diisocyanate in the second step. The introduction of polyether polyols was important because they could be produced for less than half the cost of polyester polyols. In 1958 and 1959, silicone surfactants and tin catalysts were developed that allowed the production of polyurethane foams in a one-step process. This again decreased the price of the flexible foam and opened up the large furniture and mattress markets.

The yearly production of polyurethane foams has grown steadily since 1960. One reason for this is the versatility of polyurethane chemistry and technology. Polyurethane foam formulations can be varied to produce foams with a wide range of physical properties from very soft, low modulus foams weighing less than a pound per cubic foot to high modulus microcellular foams weighing sixty pounds per cubic foot. Polyurethane foams also have the advantage that they can cure at room temperature and that all formulation components are liquids at room temperature. This allows them to be used in many 'in situ' applications such as polymerization of carpet backing right on the carpet.

Some of the more recent advances in polyurethane technology are reaction-injection molding $(\mathrm{R} I M)$ and the development of high-resilience (HR) foams. In the RIM process, liquid streams of the formulation components are pumped stoichiometrically together and sprayed into a 
mold. The polymerization process results in a polymeric material in no need of further processing. Some RIM products are solid or microcellular automobile bumpers, foamed automobile dash panels with an integral skin, and molded foams for high quality automobile seating. HR foams are low density foams in which the modulus is increased by modifying the polyol or adding crosslinkers to the foam formulation. The lower density represents a materials savings to the manufacturer. They are made both as slabstock and molded foams. Other areas of research are aimed at improving catalysts, surfactants, polyols, fillers, and other formulation components.

More fundamental studies of polyurethane foams are focused mainly on the kinetics of the foaming process and how that affects both the macroscopic and microscopic morphologies of the foam. Another area of work concerns separating the physical properties into a part derived from the bulk modulus of the material and a part due to the cell structure. A more thorough review of research efforts is presented later.

\subsection{CHEMISTRY OF WATER-BLOWN POLYURETHANE FOAMS}

There are three primary reactions in the manufacture of flexible water-blown polyurethane foams. The first is the reaction of an isocyanate group with an hydroxyl group to yield a urethane (Fig. 1.1a). In the foam forming process, this reaction is responsible for 
joining oligomeric polyols. These polyols are usually diols or triols with equivalent weights of from 500 to 2000. When the polyols or isocyanates have a functionality of greater than two, conversion of this reaction results in the formation of a gel.

The second reaction is between an isocyanate group and a primary amine (Fig. 1.1b). The product is a substituted urea. In an uncatalyzed system, this reaction is about a thousand times faster than the hydroxyl-isocyanate reaction. The importance of this reaction is coupled with the third primary reaction.

The third reaction is between an isocyanate group and water (Fig. 1.1c). The product is an unstable carbamic acid which decomposes into a primary amine and carbon dioxide. The resultant primary amine, as stated above, can react with another isocyanate group to form a substituted urea. Together these reactions provide the principal source of gas for the blowing of the foam and also chain extend the polymeric sequence of hydrogen-bonding urea groups.

All of these reactions are exothermic. The reaction of an isocyanate group with a secondary hydroxyl liberates about 24 kcal per mole. The reaction of two isocyanate groups with one part water liberates about $45 \mathrm{kcal}$ per mole of water reacted. These heats of reaction are based on phenyl isocyanate and will change with isocyanate structure and the active hydrogen of the donating material. Commercial water-blown foams are formulated with from 2 to 5 parts by weight water per hundred parts polyol (pph). Based on a polyol 
equivalent weight of 1000 and complete reaction of isocyanate with water and polyol active hydrogen, urea formation (blowing and chain extension reactions) results in 68 percent of the heat liberated for a 2 pph water formulation and 84 percent for a 5 pph water formulation. The total heat liberated is responsible for a 75 to $150 \mathrm{C}$ increase in the temperature of the foam-mix during rise and curing.

In the production of polyurethane foams, there are two important secondary reactions which involve the products of the above reactions. The active hydrogen on a urethane linkage can react with an isocyanate group to form an allophanate (Fig. 1.2a). The active hydrogens on a urea group can react with an isocyanate group to form a biuret (Fig. 1.2b). These reactions result in branch points from the polymer 'main chain' and thus introduce crosslinks into the system. Biuret formation is of more importance in flexible foams because the urea hydrogens are more reactive with isocyanate groups than the active hydrogens on urethane linkages. Biuret formation is also catalyzed by a tertiary amine which is widely used as a blowing reaction catalyst in flexible foam manufacture. Temperatures of greater than $120 \mathrm{C}$ are necessary for significant allophanate formation in uncatalyzed systems and the catalysts used in foam formulations do not generally promote this reaction.

\subsection{FORMULATION COMPONENTS}


FOR MANUFACTURE OF WATER-BLOWN POLYURETHANE FOAMS

There are five basic ingredients in a formulation for a flexible polyurethane foam. They are the polyol, isocyanate, water and auxilliary blowing agents, catalysts, and surfactant. Other ingredients such as pigments, flame retardants and stabilizers are added for particular applications. The most common materials used in the manufacture of water-blown flexible slabstock foams for each formulation component and their importance in processing and final product properties are discussed in detail below.

\subsubsection{Polyol}

Nearly all of the flexible polyurethane foams produced are based on either polyether or polyester polyols. Polyether polyols are much cheaper than polyester polyols and are more resistant to hydrolysis. Polyester polyols are more resistant to oxidation and yield foams with higher compressive strength, stiffness, and tensile strength (2).

\section{Polyethers}

The polyethers that are most widely used are those derived from the cyclic oxides of propylene and ethylene. These polyethers yield foams with the best performance to cost ratio. Polyethylene oxide (PEO) polyols are hydrophilic and have poor hydrolytic stability. Use of PEO polyols alone is limited. Polypropylene oxide (PPO) is hydrophobic and has good hydrolytic stability. Most polyether polyols 
used in the production of flexible foams are either PPO polyols or a copolymer of PPO and PEO. The polyols are usually diols or triols with equivalent weights from 500 to 2000 .

Polypropylene oxide and polyethylene oxide are produced through a base catalyzed ring-opening polymerization in which the initiator is usually glycerine for a triol or dipropylene glycol for a diol. PPO obtained in this manner is atactic. With PPO, the ring opening can occur at either the 1 or 2 positions. Attack favors the first carbon by about ten to one. The result is a backbone with about ten percent of the repeat units joining head-to-head, and a chain with about ten percent of the end groups primary hydroxyls and ninety percent secondary hydroxyls. Polymerization of PPO is also subject to a side reaction producing unsaturated allyl end groups. This side reaction increases with polymerization temperature and the probability of it occuring increases with degree of polymerization. The unsaturated end groups are unreactive with isocyanates, so as the desired molecular weight of the PPO polyol increases, the functionality of the polyol will decrease.

The polymerization of ethylene oxide is not subject to these problems. There is not a side reaction which significantly reduces the functionality of the resultant polymer and all of the end groups are primary hydroxyls. The manufacture of these polyols is discussed in more detail elsewhere $(1,2,4)$. 
The most commonly used polyether polyols are copolymers of PO and about fifteen or less percent EO. EO is incorporated into the chain either randomly or more commonly as blocks capping the PO polyols. When added randomly the EO increases the solubility of water in the polyol and increases the primary hydroxyl content when, by chance, an EO unit terminates the polyol. Capping PO polyols with EO blocks also increases the solubility of water in the polyol and produces polyols with higher primary hydroxyl content. The repeat units for these polyethers are shown in Figure 1.3.

\section{Polyesters}

Some of the differences between foams made with polyesters and polyethers are due to the presence of the ester carbonyl groups. The ester carbonyl has increased polarity as compared to the ether oxygen. This allows it to form hydrogen bonds with urea and urethane linkages and results in a foam with greater compressive strength, stiffness, and tensile strength. The ester linkage can resist oxidation and solvents better than the ether oxygen. Also, in the synthesis of polyester polyols there is no loss in functionality as in PPO polyols. This again adds to the strength of the polyester foams relative to polyether foams. The polyester carbonyl is, however, susceptable to hydrolysis and foam properties will decline rapidly in humid environments. The polyester polyols are much more expensive than polyether polyols and as a result, polyester-based flexible foams make up less than ten percent of the market. 
Lightly branched poly(oxydiethylene adipates) are the most widely used polyester polyols in flexible foam manufacture (Fig. 1.4). They are produced by the direct condensation of diethylene glycol with adipic acid and a branching agent. For improved cold weather performance, the glass transition is lowered by replacing diethylene glycol with a mixture of diols or by incorporating segments of polycaprolactones into the polyester chains.

\subsubsection{Isocyanates}

The isocyanates used in the production of flexible foams are 2,4and 2,6-toluene diisocyanate (TDI), 4,4-diphenylmethane diisocyanate (MDI), and polymeric MDI. The molecular structures of these isocyanates are shown in Figure 1.5. In addition to the pure isocyanates, a wide range of mixtures and derivatives are used.

In the early years of the flexible foam industry, TDI was used almost exclusively. TDI is still the most widely used isocyanate though polymeric $M D I$ and mixtures of TDI and MDI are becoming competitive in some areas such as self-skinning foams, high quality cushioning foams, and high resilience foams. MDI is the preferred isocyanate in the production of rigid and semi-rigid foams and in the production of microcellular elastomers in RIM processes.

Usually a five to ten percent excess of the stoichiometrically required amount of isocyanate is added to the foam formulation. This is expressed in terms of an isocyanate index where an index of 105 
means 5 percent over stoichiometry. Adding excess isocyanate increases both the stiffness of the foam and its compression set.

\section{$T D I$}

TDI is manufactured from toluene by nitration with mixed nitric and sulfuric acids, catalytic reduction with hydrogen to the diamine, followed by phosgenation and distillation. With removal of the 2,3and 3,4-isomers a product is obtained that is roughly 80 percent 2,4and 20 percent 2,6-isomer. By nitrating toluene to the mononitrotoluene stage, separating the 2-nitrotoluene and 4-nitrotoluene by distillation, and then continuing the nitration, phosgenation, and distillation process on 2-nitrotoluene, an isomeric mixture is obtained that is roughly 65 percent 2,4-isomer and 35 percent 2,6-isomer. A more detailed description of the process is presented elsewhere (7). Most TDI sold today is the 80:20 mixture of isomers. The 65:35 mix yields a higher load bearing (HLB) foam and is sometimes used as an admixture to the 80:20 isomer mix. The increased concentration of 2,6-isomer results in a harder foam because the reacted 2,6-isomers are more sterically hindered than reacted 2,4-isomers (6).

TDI is a colorless liquid at room temperature and has a boiling point of $120 \mathrm{C}$. It is a hazardous chemical and requires special handling considerations. It is fairly volatile and at low concentrations in air causes eye and respiratory irritations. The isocyanate groups are highly reactive with active hydrogens such as those in water and 
hydroxyl groups. Due to steric hindrance, isocyanate groups para to the methyl group are ten times more reactive than isocyanate groups ortho to the methyl group at $25 \mathrm{C}$. With increasing temperatures, the effect of steric hindrance is reduced. At temperatures of $100 \mathrm{C}$, steric hindrance is no longer a factor (9).

\section{Urethane Modified TDI}

In an uncatalyzed system, the blowing reaction (isocyanate-water) is faster than the gelling reaction (isocyanate-hydroxyl). Chain extension and blowing precede the large increase in viscosity that occurs when the polyols are joined and a rising foam will collapse before it can support itself. Before catalysts were developed that could adequately balance these reactions, polyurethane foams were made in a two-step process. In the first step, the polyol is capped by reacting it with excess diisocyanate. The capped polyol is referred to as a prepolymer. In the second step, the prepolymer is reacted with water and isocyanate to form the foam. Today, polymers similar to these prepolymers are used as adhesives and surface coatings and in the formulation of cold-cure molded foams. In cold-cure foams, some of the polyol is prereacted with isocyanate to aid in curing and to match viscosity and improve miscibility of the formulation components .

\section{Allophanate Modified TDI}

The functionality of TDI can be increased by allophanate 
branching. One way in which this is accomplished is by reaction with ethylene glycol. At low temperatures, isocyanate groups will react with the hydroxyls of ethylene glycol. When this diisocyanate is heated in the presence of TDI, an allophanate is formed (Fig. 1.6). Catalysts and alkylating agents are available that allow this reaction to be carried out in a reasonable amount of time and hinder formation of isocyanurates. Allophanate TDI is used in conjunction with TDI 80:20 in both slabstock and molded high resilience foam.

\section{Isocyanurate Modified TDI}

TDI, in the presence of basic catalysts, can trimerize to form isocyanurates (Fig. 1.7). Formation of isocyanurates is not reversible and isocyanurates show little degradation below $270 \mathrm{C}$. Addition of this trimer to TDI $80: 20$ is one of the more common ways of increasing isocyanate functionality. Isocyanurates can also form during the production of flexible foams when basic catalysts are used.

\section{$M D I$}

Pure MDI is obtained by distillation from a diisocyanate-rich grade of polymeric MDI. It is a solid with a melting point of $37 \mathrm{C}$ and has a tendency to dimerize at room temperature. MDI has a much lower vapor pressure than TDI and as a result is less of an eye and respiratory irritant. The sterically free isocyanate groups are too reactive for use in standard foam formulations and are too expensive to compete with TDI. 
Pure MDI is highly symmetric and when chain-extended with low molecular weight diols forms well-ordered hard segments that can crystallize. The main use of pure MDI in urethane foams is in the production of microcellular elastomers.

\section{Polymeric MDI}

Polymeric MDI is the undistilled mixture of 4,4-diphenylmethane diisocyanate, the 2,4-isomer, and a distribution of tri-, tetra-, penta-, and isocyanates of slightly higher functionality. The manufacture of polymeric MDI is reviewed in detail elsewhere (7). The process starts with the reaction of aniline and formaldehyde to primary and secondary amines and water. The product is heated with excess aniline to yield polyamines. These polyamines are then separated from the water and excess aniline and phosgenated in the presence of hydrogen chloride to yield the mixture called polymeric MDI .

The mixture is characterized by viscosity and isocyanate content. High viscosity grades of polymeric MDI may be around $2000 \mathrm{mPA}$ and have an average effective functionality of 3.0. These grades are used in rigid foam processes. Grades with a viscosity around $200 \mathrm{mPa}$ and an average effective functionality of 2.7 are used to make non-waterblown self-skinning flexible and semi-rigid foams and are used in mixtures with TDI for cold-cure and high resilience foams.

\subsubsection{Blowing Agents}


The reaction of water with two isocyanate groups results in the offgasing of carbon dioxide and chain extension of the hard segment. Increasing the water content in a foam formulation while maintaining isocyanate stoichiometry requirements increases the amount of carbon dioxide given off and increases both the weight percent hard segment and the ratio of polyurea to polyurethane in the hard segment. With more carbon dioxide released, the foam is more highly blown and, as a result, is less dense. Both the increasing hard segment content and the increased hydrogen bonding due to urea linkages act to stiffen the foam. In the range of standard commercial foams (up to 5 or 6 parts of water per hundred parts polyol) the lower density and increasing hard segment compensate for each other. As a result the hardness of water-blown foams changes very little with water content. Above this water level, foam properties decline due to an exceedingly high exotherm, the presence of too much hard segment, and a foam structure of too low density.

Low boiling inert liquids are used as auxiliary blowing agents to obtain softer foams at lower densities. The boiling of these liquids also removes some of the heat released by the exothermic reactions. The most common blowing agent is the fluorocarbon, trichloromonofluoromethane. Environmental groups have expressed concern for the depletion of ozone by fluorocarbons but restrictions have not been placed on their use in flexible foam production. Should industry be forced to find an alternative blowing agent, methylene chloride is the most likely alternative. It has been used as an 
auxiliary blowing agent alone, and with fluorocarbon agents for years. The disadvantages with methylene chloride are its lower volatility, higher toxicity, and higher solubility in the foam. When dissolved in the foam methylene chloride leaves an unpleasant odor and discolors the foam. Methylene chloride is cheaper than fluorocarbon blowing agents and some of the problems associated with its use can be reduced by using delayed action catalysts.

\subsubsection{Catalysts}

The job of the catalyst system is to balance the isocyanate-water (blowing) and isocyanate-hydroxyl (gelling) reactions to ensure the production of a stable foam in the desired processing time. If the blowing reaction is over catalyzed, the foam will collapse before it can gel. If gellation occurs too quickly, the cells will not open completely and the foam will shrink. From a processing and final product viewpoint, a catalyst system should allow sufficient induction time between the mixing of ingredients and foaming, allow adjustment of this induction time, the rise time, and the curing time, make possible adjustments in the final foam properties, have no disadvantages such as toxicity or odor, or cause discoloration or increased degradation of the foam.

The most common catalyst system used in slabstock flexible foam production is a combination of a tertiary amine and a tin compound. Tin compounds are more powerful catalysts for both the blowing and gelling reactions than the tertiary amines and it was their introduction 
in 1959 that made the one-shot production process possible. At low temperatures, tin catalysts favor the gelling reaction over the blowing reaction and as a result are termed 'gelling catalysts'. At temperatures above $70 \mathrm{C}$, the selectivity between the two reactions is small (1). The first tin catalysts used were dibutyltindi(2-ethylhexoate) and dibutyltindilaureate (DBTDL). These were found to catalyze the oxidation of polyether foams at high temperatures and were replaced by stannous octoate. Stannous octoate is the most widely used tin catalyst today. DBTDL is used in low concentration with antioxidants present. The mechanism for the activation by tin catalysts is not known. For the isocyanate it is believed that the metal acts as an electron acceptor and a complex is formed through the isocyanate oxygen.

Tertiary amines also catalyze both the blowing and gelling reactions, but favor the blowing reaction because they form more stable hydrogen bonds with water. In the absence of acidic retarders, tertiary amines also catalyze chain branching through biuret formation. Some of the more common amine catalysts are shown in Figure 1.8. They are ordered from top to bottom in terms of increasing selectivity towards the blowing reaction. The activity of a tertiary amine increases with basicity and decreasing steric hindrance. The accepted mechanism is through formation of a complex in which the tertiary nitrogen donates electrons to the carbonyl carbon of the isocyanate group. The combination of a tin compound and a tertiary amine acts synergistically. A discussion of this phenomena is given in 
reference 8 .

The combination of tin and tertiary amine is the most widely used catalyst system in flexible foam manufacture but the allocation of tin compounds as gelling catalysts and tertiary amines as blowing catalysts is a misnomer. This is well illustrated by Kollmeier et al (8) and is presented in Figure 1.9. In this figure, profiles of foam rise versus reaction time are depicted for formulations with no catalysts, with only $0.2 \mathrm{pph}$ amine catalyst, with only $0.2 \mathrm{pph}$ tin catalyst, and with both tin and amine catalysts. Without catalysis, the foam rises but does not gel enough and collapses. With only the amine catalyst, the foam rises more quickly and to a greater height but again does not gel enough and hence collapses. When just the tin catalyst is used the foam rises faster than with the amine catalyst and to a greater height. This foam gels too much while rising, cell opening is not complete, and shrinkage or 'pruning' occurs while curing. The tin catalyst is therefore a strong catalyst for both the gelling and blowing reactions. When used by itself, the tin catalyst causes too much gelation relative to blowing. When both catalysts are used a stable open-celled foam is produced in even a shorter rise time. It is the opinion of Kollmeier et al that the reactivity of the formulation be specified by the tin catalyst and that fine tuning is best accomplished with the amine catalyst. Since different amines accelerate the blowing and gelling reactions in certain proportions, the foam process and physical properties, like hardness and porosity, are controlled by choice of amine. 
Most manufacturers do use the amine catalyst more as the tuning catalyst or at least as the catalyst of lower concentration as observed in published formulations $(6,1)$. There are several reasons for doing this. Amine catalysts have a lower reactivity and, as a result, finer adjustments can be made by adjusting amine content rather than tin catalyst level. Also, there is a much wider variety of amine catalysts on the market and varying amine catalysts rather than tin catalysts leaves a manufacturer more options.

The rapid growth in the manufacture of high resilience and cold cure foams has caused a growth in the catalyst market. As a result of the growing market, new catalyst development is increasing. Some areas of development include delayed action catalysts and catalysts that are bound to the foam to reduce their volatility. Delayed action catalysts improve the processability of molded foams. Catalysts of high volatility may leave the foam before it has cured. Catalysts of low volatility may slowly leave the foam causing an odor problem.

\subsubsection{Surfactants}

Surfactants are an essential part of the foam formulation because they help stabilize the rising foam and help control cell size and uniformity. The exact role a surfactant plays in the foam making process and the mechanisms by which it performs its duties are the subject of considerable controversy and will be discused in more detail when manufacturing techniques are described. A traditional view of the stabilization mechanisms present in foam formation is presented 
below.

The first step in the manufacture of polyurethane foams is the mixing of the different components of the formulation. Obtaining a homogeneous foam-mix is essential to the formation of a foam with uniform properties. One job of the surfactant is to aid in emulsifying the formulation ingredients. During agitation and spraying, air becomes dispersed in the foam-mix. These air bubbles are the nucleation centers for the carbon dioxide released in the foaming process. Surfactants influence the fineness and regularity of a foam by stabilizing this dispersion. During the rising of the foam, the surfactant stabilizes the growing bubbles by two mechanisms. The surfactant slows drainage of the cell membranes into the plateau borders by building up surface viscosity and gives elasticity to the cell membranes by a Gibbs-Marangoni effect. By this principle, a local stretching of a film or membrane will result in increased surface area. The increased surface area will be accompanied by a decrease in the surface excess concentration of surfactant and thus a higher surface tension. This local higher surface tension will stabilize the film or membrane until surfactant molecules can diffuse to the area of increased surface tension. While surfactant molecules are diffusing to the stretched area, the film has a chance to develop a more uniform and stable thickness. Finally, surfactants are believed to play a role in the timing and degree of cell opening.

Despite the controversy over the role of surfactants in foam 
making, manufacturers are able to make very successful surfactants and tailor them for use in particular foam systems. These modern-day surfactants are polysiloxane-polyoxyalkene block and graft copolymers. Two basic structures are used. One structure is comb-like with a linear polydimethylsiloxane backbone and polyether grafts. The other structure resembles a three-armed star with polydimethylsiloxane blocks comprising the inner portion of the arms and polyether blocks attached to the ends of these blocks. The bonding of the polyether blocks to the siloxane blocks can be through either a $\mathrm{Si}-\mathrm{C}$ bond or a Si-O-C linkage. The Si-O-C linked copolymers are stable in neutral or basic environments but are hydrolyzed in the presence of Lewis acids such as tin catalysts or mineral acids. The Si-C linked copolymers are chemically stable in both basic and slighlty acidic environments. Use of 'hydrolyzable' silicone surfactants used to be widespread in flexible foam manufacture but now mostly 'nonhydrolyzable' surfactants are used due to less storage problems and better reproducibility in manufacture.

The surfactants are tailored for specific applications by adjusting the lengths of the siloxane and ether blocks, the number of grafts on comb structures, the ratio of PPO to PEO in the polyether blocks, and by changing the end groups on the polyether blocks. In general, the ratio of siloxane to ether controls the activity of the surfactant and the ratio of PEO to PPO controls the solubility of the surfactant in the foam-mix . 
The composition, molecular weight, and topology of surfactants change dramatically with manufacturer and intended use. Rough size estimates are silicone blocks of 1000 to 3000 molecular weight and representing 15 to 40 percent of total weight. In graft copolymers there are usually only 3 to $5 \mathrm{grafts}$ so that these surfactants resemble to some extent the star copolymers. The polyether grafts contain inner PEO blocks and PPO end blocks. The PPO block comprises from 50 to 70 percent of the polyether blocks $(1,5,20,21)$.

\subsection{FOAM MANUFACTURE}

In the previous sections, typical formulation components for a flexible slabstock foam were presented. Slabstock foam is made in the form of a large bun or slab in either a continuous or batch operation. Modern technology allows the production of slabs eight to ten feet wide and five or so feet high with uniform properties throughout and very little waste. Furniture cushions, mattresses, and other products are carved from these slabs. In the paragraphs that follow the manufacture of slabstock flexible foam is presented in terms of the sequence of reactions that take place and the resultant morphology. More details about the machinery used to make these foams are available in references 1,4 , and 5 .

The formulation ingredients-polyol, isocyanate, surfactant, water and blowing agent, catalysts, and additives are stored under a dry air 
or nitrogen atmosphere and at a certain temperature and pressure to control the volume of dissolved gas. These streams are all liquids at room temperature and are pumped in the correct proportions into a mixing head. The low pressure behind the rotating agitator blade causes nucleation by expelling the dissolved gas. The extent of nucleation is controlled by the pressure in the mixing head, the rate of agitation, and the amount of dissolved gas. Air is often added to one of the formulation components or in the mixing head to increase the level of nucleation. The surfactant stabilizes this dispersion of air in the foam-mix and in this way controls cell size and uniformity.

The importance of agitation rate and air loading on foam properties has not been extensively reported in the literature. This is probably because these are processing variables rather than formulation variables and are influenced more by the types of processing equipment than by changes in the chemical compostion of the foam-mix. In one study, Baumhakel found that at the proper agitation rates a foam with fine, uniform, completely open cells was formed (10). At lower agitation rates, the cells were larger, partially closed, and had thicker struts. At higher agitation rates a foam with a fine, uniform, open cell structure was again produced but some of the struts were torn and macroscopic splits were formed.

The stabilized air dispersion in foam-mix is then sprayed on to a moving conveyor or into the bottom of some type of walled support. After a few seconds the foam-mix will turn opaque and start to fizz. 
This induction time between mixing and the first signs of foaming is called the 'cream time.' The length of the cream time is an important parameter in foam processing especially in the production of molded foams and can be controlled by catalyst selection. Generally, longer cream times allow more of the dispersed air bubbles or nucleation centers to coalesce resulting in foams with larger cells.

As the water and isocyanate react, carbon dioxide is given off. The pressure in the nucleation bubbles increases and the foam-mix expands. When the volume of the air bubbles becomes large compared to the liquid, the contact area between the adjacent bubbles becomes a membrane. The line of contact between three membranes forms a rib. These ribs form polyhedrons. In foams with uniform cell structure dodecahedrons are usually formed with pentagonal windows. As more carbon dioxide is given off, the bubbles continue to grow and the membranes thin and flow into the ribs. This process continues until the cells rupture. Cell rupture is believed not to be controlled by membrane thinning, but by some undefined chemical or morphological changes in the rising foam. This is discussed in detail later.

The liquid foam-mix takes from 60 to 180 seconds after initial mixing to expand and reach cell rupture. During this time it undergoes a 75 to $150 \mathrm{C}$ exotherm depending on the water content and isocyanate index of the formulation.

At cell rupture, up to thirty percent of the original isocyanate is unreacted and the foam is low strength. The foam matrix is very 
viscous and diffusion controls the rate at which the unreacted isocyanate reacts with polyols, water, and urea and urethane hydrogens. These reactions increase the temperature of the selfinsulating foam block to its maximum temperature thirty to sixty minutes after rise. The block remains near this temperature for two to eight hours after foam rise depending on block size, storage conditions, and excess isocyanate concentration. Large blocks of foam require about 48 hours to cure under ambient conditions. Smaller blocks of foam, which do not reach and maintain the same interior temperatures, must often cure for seven days before properties stabilize.

\subsection{RECENT WORK}

The high rates of chemical reactions, large exotherms, high rates of volume and viscosity changes, and the heterogenity of the reacting mixtures have limited the research on polyurethane foams. Polyurethane foams are a large commercial product and most of the published research is aimed at selling new and improved commercially available formulation components rather than explaining why these new components work. Within the last ten years, some researchers have taken a more fundamental approach to foam research. Some of the work pertinent to the authors thesis is summarized below. 
The properties of foams depend both on the macroscopic cell structure of the foam and on the microscopic morphology of the ribs or struts. The morphology of the ribs is believed to be phase separated as in thermoplastic urethane elastomers. Thermoplastic urethane elastomers, like polyurethane foams, are comprised of atactic polyether or polyester oligomers joined by reaction with chain-extended diisocyanates. The diisocyanate is MDI and it is often chain-extended with butanediol. The chain-extended MDI segments are incompatible with the polyester or polyether oligomers and tend to aggregate and form domains. The chain-extended MDI segments can also crystallize. When the system contains more than about sixty percent polyester or polyether (soft segment), these flexible chains form a continuous matrix. The chain-extended MDI segments (hard segments) form domains dispersed in the soft segment matrix. These domains are partially crystalline and are well below their glass transition at service temperatures. The soft segment has a low glass transition temperature and is highly flexible at service temperatures. The result of this morphology is a material that shows elastomeric properties without being chemically crosslinked. The ends of the soft segment chains are physically crosslinked by the formation of the hard segment domains.

Phase-separation in foams is restricted compared to typical MDIbutane diol elastomers by the use of tri- or higher functional polyols or isocyanates, by possible formation of biurets and allophanates to disrupt hard segment packing, and by use of a mixture of TDI isomers that will not allow hard segment crystallization. The phase separation 
issue is further clouded by the fact that chain extension and gelling occur simultaneously in the foam making process. The focus of much recent work is the sequence of reactions during the manufacture of conventional slabstock foam. A relative measure of the chain extension reaction rate to those for gelling, and allophanate and biuret formation may be useful in evalutating the extent of phase separation in foams.

The sequence of reactions was studied by a number of investigators using FTIR (11-15). All investigators observed a growing urea carbonyl absorption at 1715 wavenumbers early in the reaction. When about half of the rise height or $80 \mathrm{C}$ was reached, this peak shifted to 1640 wavenumbers and continued to grow. Model studies on diphenyl urea indicated that in good solvents the urea carbonyl absorption was observed at 1715 wavenumbers and in poor solvents it was observed at 1640 wavenumbers (11). Therefore, in the formation of polyurethane foams a point is reached at which the polyurea is no longer soluble in the foam-mix and precipates out.

Rossmy and coworkers (15) hypothesized that the more reactive para isocyanate groups react first and that when these groups have all reacted, the ortho groups must react leading to larger products which precipitate out. Their statement is pure speculation, but it may be sound. The formation of polyurea is a step-growth process. In all step-growth polymerizations a point is reached where monomer concentration is depleted and oligomers must react with one another. At this point, the molecular weight increases exponentially with 
further reaction. The solubility of polymers generally decreases with increasing molecular weight. Therefore urea precipitation could be triggered by an increase in polyurea molecular weight.

FTIR investigations revealed other important facts about the sequence of reactions. Hauptman and Zarkov showed that the waterTDI reaction is the limiting factor in the blowing and chain extension reaction sequence and not the decomposition of carbamic acid or the amine-TDI reaction $(11,13)$. Hauptman also reported no traces of allophanate or biuret formation before the foam-mix reached $80 \mathrm{C}$. Bailey and Critchfield noted that the relative absorbance of urea increased quickly within the first five minutes after mixing (remember only a two to three minute rise time) and then remained constant (12). The relative absorbance of urethane increased more slowly over the first five or ten minutes but then continued to increase at a slow and steady rate for at least thirty more minutes. Though the measurements were not quantitative, it can be said that urea formation took place more quickly and essentially stopped and that significant urethane formation took place for at least forty minutes after mixing. Rossmy and coworkers confirmed the unimportance of urethane formation in the early part of foam formation by synthesizing foams with non reactive polyols and by analyzing the unreacted components sampled and quenched at various times during the reaction (15). Plots of foam temperature versus foam height for the foams made with reactive and unreactive polyols were nearly identical indicating that the heat generated by urethane formation early in the reaction is 
negligible. The ratio of isocyanate group to water consumption was $2: 1$ in the early part of foam formation as required by the blowing and chain extension reaction sequence, also indicating that urethane formation is not significant in the early stages of the reaction.

Van Gheluwe and LeRoux studied the sequence of reactions in polyurethane foams by measuring the temperature of the foam versus time and the change in temperature or first derivative with time (16). In every foam formulation studied, two maximums were observed in the temperature-time derivative plots. By modifying the reactivity of the polyols and changing catalyst concentrations they were able to assign the early peak to a maximum rate of the urea reaction and the latter peak to a maximum rate of the urethane reaction. This again shows the predominance of urea formation in the early stages of foam formation.

Rowton developed a simple, qualitative technique for measuring the rigidity of a rising foam (17). The test is called a BB-drop test and consists of dropping BB's into the rising foam at different time intervals from a fixed height above the rising foam surface. When the foam has cured it is cut open and the height of the $B B$ above the bottom is an indicator of the integrity of the foam when the $B B$ was dropped. Bailey and Critchfield used this technique in conjunction with their FTIR work (12). The BB's sank through the rising foam until the precipitation of urea occured. Then the dropped BB's were supported by the foam indicating that the integrity of the foam was 
sharply increased by urea precipitation.

Rossmy and coworkers investigated the urea precipitation phenomenon more carefully $(14,15,18-20)$. By adding a defoaming agent to a foam formulation they showed that urea precipitation turned a clear foam-mix opaque. No analysis of the precipitate was reported. The temperature of urea precipitation increased with water content, increasing TDI-index, and increasing 2,4-isomer content. These changes all increase the reactivity of the foam-mix and this could explain the higher urea precipitation temperatures. They also noted that cell rupture always occured just after urea precipitation. In light of the above observations they hypothesized that precipitation of polyurea destabilizes the foam-mix and leads to cell opening. The surfactant aids in stabilizing the foam by incorporating the urea precipitate into the foam matrix. This adds integrity to the foam. The performance of the surfactant then depends on the molecular weight of the polyether segment and in particular the ratio of hydrophilic, polyurea-liking PEO block to the hydrophobic PPO block.

Studies of the sequence of reactions reveal that some sort of urea precipitation occurs during foam manufacture resulting in a two phase system. In spite of this not much can be found in the literature on the morphology of the cell struts or membrane-like windows of these foams. Wilkes studied the morphology of a series of conventional and high resilience foams with SAXS and DSC (39). The conventional foams did not show a Bragg peak (evidence of phase separation) until 
the polyol was replaced with a polyol containing twenty weight percent polyurea grafts. The $3500 \mathrm{MW}$, trifunctional, secondary hydroxyl terminated polyols used in the conventional foams were replaced with a $6000 \mathrm{MW}$, trifunctional, primary hydroxyl terminated polyol in the high resilient foam. Also a lower water level and weaker surfactant were used. These foams showed a distinctive Bragg peak corresponding to spacings in the range of 5 to 20 nanometers. DSC showed that PEO crystallization was not present and it was concluded that the Bragg peaks were the result of phase separation. Qualitatively it could be stated that the higher polyol MW and the weaker surfactant in the high resilient foams did more to allow phase separation to occur in this system than the presence of primary hydroxyls did to prevent it.

\subsection{SUMMARY}

A formulation for a conventional water-blown flexible polyurethane foam consists of five basic ingredients. Polyols are usually di- or trifunctional with equivalent weights of 500 to 2000 . They are usually PPO or PPO capped with PEO. The isocyanate source is most often an $80: 20$ mixture of the 2,4- and 2,6-isomers of toluene diisocyanate. For most formulations isocyanate indices of from 105 to 110 are used. The foam is blown by reaction of water and an isocyanate group giving off carbon dioxide. Flexible foam formulations can vary from 2 to 6 parts by weight water per hundred parts polyol. The catalyst system 
consists of both a tin-based gelling catalyst and a tertiary amine-based blowing catalyst. The surfactant is a low molecular weight block or graft copolymer with a siloxane block and PPO and PEO blocks or grafts.

Foam manufacture takes place in less than five minutes though foam properties will not stabilize until after two or more days of curing. The foam forming process involves competing blowing and gelling reactions, and the stabilization of a rising foam through large viscosity changes and a 75 to $150 \mathrm{C}$ exotherm. The foam-mix is not homogeneous and the precipitation of urea may somehow lead to cell rupture. Evidence of urea precipitation also indicates that some type of phase separated morphology exists in the foam. The existence of phase separated domains has been observed in some high resilience foams using SAXS. To the author's knowledge, no thorough investigation into the morphology of polyurethane foams is in the literature. 


\section{CHAPTER TWO: SMALL ANGLE SCATTERING THEORY}

\subsection{INTRODUCTION TO SAXS}

Polyurethane foams are segmented copolymers comprised of polyether and polyurea blocks or segments. The incompatibility of the ether and urea blocks would drive a system of these segmented molecules to phase separation and the formation of discrete polyurea and polyether domains. Many polyurethane foams, however, are made with trifunctional polyethers which form a network on reaching a high degree of polymerization. Molecules incorporated into a network cannot diffuse as easily to form phase separated domains. The technique of small angle $x$-ray scattering (SAXS) can often be used to detect the presence of phase separation in segmented copolymers and to characterize many of the morphological features of this domain texture.

A short overview of small angle $x$-ray scattering theory is presented in this chapter. SAXS comprises a large portion of the author's research work and an introduction is necessary for interpretation of the data. In this first section, the origin of SAXS is discussed in terms of the basic interactions between $x$-rays and matter, and Bragg's law. In the second section, the theory behind the SAXS analyses used to characterize the polyurethane foams and 
plaques is presented. This review has been principally derived from references 32 through 38 .

\subsubsection{Interactions of X-rays with Matter}

$X$-rays are electromagnetic waves with wavelengths shorter than ultraviolet radiation and longer than gamma radiation. These wavelengths range from 0.01 nanometers to 10 nanometers. When they are passed through matter they interact with the atoms in ways which bring out the dual particle-wave nature of electromagnetic radiation. $X$-ray photons collide with inner shell electrons and expel them from the atom. This raises the energy level of the atom. An electron drops from an outer shell to fill the inner shell vacancy. As the electron falls to the lower energy shell, radiation is released. This radiation is characteristic of the shell-to-shell transition and can be used to identify the atom. The expelled electron is called a photo electron and the characteristic radiation is called fluorescent radiation. This interaction is not important in $x$-ray scattering except that it dissipates energy from the incident beam.

X-ray photons can also collide with loosely bound electrons. As a result of the collision, the incident beam is deflected or scattered and loses some of its energy to the electron. Because the $x$-ray loses energy its wavelength increases and the phase relationship with the incident beam is lost. Therefore this scattering cannot result in diffraction. This type of scattering is called Compton or incoherent scattering. It is unimportant in small angle scattering and can be 
compensated for in wide angle scattering.

Finally, $x$-rays induce motion in the electrons they traverse. Acceleration and deceleration of charges generate electromagnetic radiation. Therefore, electrons in a specimen will accelerate with the varying electric field of the propagating electromagnetic $x$-ray wave and become emitters of $x$-rays of the same wavelength and with a phase relationship to the incident beam. This type of scattering is called coherent or Thomson scattering and is the basis for $x$-ray diffraction .

When $x$-rays pass through matter their energy is dissipated by all three of the above mechanisms: expulsion of electrons; incoherent scattering; and coherent scattering. The energy or intensity of the $x$-rays which pass through the sample is the transmitted intensity. Energy lost when passing through the sample is said to be absorbed by the sample. The fractional decrease of the intensity of an $x$-ray beam as it passes through matter is proportional to the thickness of the matter.

$$
\frac{d I}{I}=-\mu d t
$$

In the above expression, 1 is the intensity, $t$ is the thickness of the matter, and $\mu$ is the linear absorption coefficient. It is dependent on the composition of the absorbing material, its density, and the wavelength of the $x$-ray beam. Integration of the above expression removes the derivative and results in a more common expression for 
defining the linear absorption coefficient.

$$
I(t)=I(0) \exp (-\mu t)
$$

Linear absorption coefficients are tabulated for the elements at the most commonly used $x$-ray wavelengths. For compounds the absorption coefficient is calculated on a weight average basis from the coefficients for the elements.

In SAXS the incident beam is alligned perpendicular to a planar (film) sample and the scattering is measured at small angles from the transmitted beam. When thin samples are used the volume of electrons that sees and scatters the $x$-ray beam is small and the measured scattering is of low intensity. When thicker samples are used, the scattering from the initially encountered volume of electrons is absorbed by the remaining thickness of the sample. Combination of these two effects results in an optimal sample thickness for SAXS measurements of $1 / \mu$. For metals or other materials with high atomic numbers (higher electron density) the optimal thickness is about a tenth of a millimeter. Polymeric materials comprised of low atomic number elements such as hydrogen, carbon, nitrogen, and oxygen have optimal thicknesses of from 0.5 to 2.0 millimeters.

\subsubsection{Diffraction and Bragg's Law}

In an x-ray experiment, the sample can be thought of as an ordered or disordered assembly of atoms in which every electron is emitting $x$-rays in all directions and in phase with the incident beam 
(coherent scattering). In the forward direction, the scattered intensity would be the sum of the scattering from all of the electrons. As one moves to angles other than zero degrees, the pathlength of an $x$-ray through the sample, to a scattering electron, and out to a detector, changes with the location of the electron in the material (Fig. 2.1). The differences in pathlengths translate to phase shifts in the scattered $x$-ray waves relative to one another. For scattering centers separated by a distance $d$, the phase shift at a scattering angle $\theta$ is given by Bragg's law.

$$
n \lambda=2 d \sin \theta
$$

The phase shift is expressed by $n \lambda$, where $\lambda$ is the $x$-ray wavelength and $n$ is the number of wavelengths in the phase shift. At angles where $n$ assumes an integer value the phase shift is an even number of wavelengths and completely constructive interference occurs. At other angles, the phase shift is not an even number of wavelengths and varying degrees of constructive and destructive interference occur.

Bragg's law was originally derived for wide angle diffraction by crystals. These experiments are run in the reflection geometry, and the variable $d$ is the interplanar spacing (Fig. 2.2). Because of the long range ordering in crystals, the sum of the scattering from all the atoms in the lattice is completely destructive except for when Bragg's law is satisfied. A wide angle scan of a crystal will therefore show essentially no scattering except for peaks where Bragg's law is 
satisfied. (In SAXS experiments the transmission geometry is used and the measured angle is actually twice the Bragg angle (Fig. 2.3).)

\subsubsection{Origin of Small Angle Scattering}

In a single crystal, the unit cell configuration is repeated over and over again in all directions. This long range order leads to complete destructive interference except where Bragg's law is satified. Bragg's law interferences are the result of the reoccurances of fixed distances between scattering centers and arise from interplanar distances in crystals. A wide angle $x$-ray diffraction profile for a single crystal shows essentially no scattering except for Bragg's law peaks (Fig. 2.4a).

In a liquid or amorphous solid both intramolecular and intermolecular spacings can lead to diffraction. The distances between the atoms of a molecule are not fixed but vibrate about a mean value. The distances between molecules are not fixed either but vary with the balance of the repulsive and the attractive forces between them. These are reflected in a wide angle diffraction pattern by broadened or diffuse interference peaks (Fig. 2.4b). For many amorphous systems more than one diffuse peak is observed.

In monatomic gases, there is no repetitive interatomic distance to give rise to an interference peak, nor is there a narrow distribution of interatomic distances to yield a diffuse peak. The lack of longrange order minimizes destructive interference. The result is a 
scattering profile that represents the sum of the scattering by individual atoms (Fig. 2.4c). This is called particle scattering.

Intensity profiles in small angle $x$-ray scattering, (SAXS), contain contributions from both interference and particle scattering. These contributions arise from inhomogeneities on the 2 to 100 nanometer level. Such inhomogeneities could exist in colloidal dispersions, as phase separated domains in block or segmented copolymers, as crystalline regions in semicrystalline polymers, or as voids in a solid. In a homogeneous system, there is destructive interference and no scattering is present in the small angle region. In a very dilute dispersion of one solid in another, scattering from the particles is important and there is no correlation between the widely separated particles. When the concentration of the dispersed phase is increased, both particle and interference scattering are present.

The SAXS technique can distinguish features of roughly 2 to 100 nanometer level. Particles of these dimensions are generally not all of the same size and shape and interparticle distances are bound to vary. The particle and interference scattering, in addition to being superimposed, become more diffuse in nature. Information is obtained from the diffuse scattering profile by comparison with theoretical expressions for scattering from model systems. Some methods of analysis are discussed in the next section. 
The coherent scattering from a single electron is given by the Thomson equation

$$
I(k)=I_{0} e_{e}\left(\frac{1}{a^{2}}\right)\left(\frac{1+\cos ^{2} \theta}{2}\right)
$$

where $l_{0}$ is the incident intensity, $a$ is the sample-to-detector distance, and $i_{e}$ is a constant called the Thomson scattering factor and involves the mass, charge, and energy aspects of the scattering from a single electron. The term containing the cosine function is the polarization factor and is approximately equal to one for SAXS. In the theory that follows the right hand side of the Thomson equation is set equal to one in order to simplify the equations. When the relationship between the measured intensity and the number of scattering electrons is needed, the electron scattering can again be calculated. Intensity measurements adjusted for electron scattering are called absolute intensity measurements.

\subsubsection{Relationship Between Sample and Scattering Amplitude}

Every electron in the sample that is irradiated by the incident beam emits coherent $x$-rays. The amplitude is the same for each electron and is, for convenience, set equal to one. At any angle the amplitude of the scattered wave is the sum of the amplitudes from all the scattering electrons. The amplitude of the scattered wave changes due to the amount of constructive and destructive interference at 
different scattering angles.

The amplitude of a scattered wave can be expressed in complex form by

$$
A=\sum_{n=1}^{n} \exp (-i \phi n)
$$

where $\phi$ is the phase difference between waves. The phase difference can be expressed in terms of the path difference by

$$
\phi=\frac{2 \pi}{\lambda} \delta
$$

where $\lambda$ is the $x$-ray wavelength and $\delta$ is the path difference. In Figure 2.5, two scattering centers separated by a vector $r$ are scattering incident radiation Ao along $A s$. The lines $\mathrm{Oa}$ and $\mathrm{Ob}$ are perpendicular to the propagation direction of the incident and scattered waves. The path difference between waves scattered by centers $O$ and $X$ is

$$
\delta=a x+b x
$$

This path difference can also be calculated by extending the incident and scattered wave paths and projecting the vector $r$ on to them

$$
\begin{aligned}
\delta & =0 d+0 c \\
\delta & =\bar{r} \cdot \bar{A}_{0}+\bar{r} \cdot\left(-\bar{A}_{S}\right) \\
\delta & =-\bar{r} \cdot\left(\bar{A}_{S}-\bar{A}_{0}\right)
\end{aligned}
$$


The phase difference can now be expressed by

$$
\Phi=\frac{2 \pi}{\lambda}\left(\bar{A}_{S}-\bar{A}_{0}\right) \cdot \bar{r}
$$

When the phase difference is a multiple of $2 \pi$, Bragg's law is satisfied.

$$
\begin{gathered}
\Phi=n 2 \pi=\frac{2 \pi}{\lambda}\left(\bar{A}_{s}-\bar{A}_{0}\right) \cdot \bar{r} \\
n=\frac{1}{\lambda}\left(\bar{A}_{S}-\bar{A}_{0}\right) \cdot \bar{r}
\end{gathered}
$$

In Bragg's law (eqn. 2.3), let $d=r$. Hence

$$
n=\frac{1}{\lambda}(2 \sin \theta) \bar{r}
$$

Combination of equations (10) and (11) results in

$$
\left(\bar{A}_{S}-\bar{A}_{0}\right)=2 \sin \theta
$$

The phase difference can be rewritten as

$$
\phi=\frac{2 \pi}{\lambda}(2 \sin \theta) \cdot \bar{r}
$$

The term $4 \pi \sin \theta / \lambda$ is referred to as the scattering vector and is denoted by $k$. Through Bragg's law, the scattering vector is reciprocally related to $d$, the spacing between the scattering centers.

$$
\kappa=\frac{4 \pi}{\lambda} \sin \theta=\frac{2 \pi}{d}
$$

The scattering amplitude in any direction for $\mathrm{N}$ electrons can now be expressed by

$$
A=\sum_{n=1}^{n} \exp \left(-i k \bar{r}_{n}\right)
$$


The vector $r$ relates the position of scattering centers and has the units of nanometers. The vector $k$ is derived from scattered $x$-ray pathlength differences and has the units of inverse nanometers. The two vectors are reciprocally related. Since $r$ relates to the positions of the scattering centers it is said to reside in physical-space or realspace. The scattering vector $k$ then lies in reciprocal-space or Fourier-space. A material that is described in real-space by a set of vectors $r$, diffracts in a manner described by the set of vectors $k$ in reciprocal-space.

In equation (2.15), the scattering amplitude is the sum of the phase factors for the $N$ scattering electrons. If $N$ is large, the discrete vectors $r$ that describe the positions of the scattering electrons are equivalent to some continuous electron density distribution $\rho(r)$. If the form of this function is known, the product of this function and the exponential phase factors for all $r$ can be integrated without being evaluated first. The summation in equation (2.15) becomes

$$
A(\kappa)=\delta_{V_{r}} \rho(\bar{r}) \exp (-i \kappa \bar{r}) d V_{r}
$$

Mathematically, the scattering amplitude is the Fourier transform of the electron density distribution. Taking the inverse Fourier transform yields the electron density distribution in terms of the integrated scattering profile.

$$
\rho(\bar{r})=\left(\frac{1}{2 \pi}\right)^{3} \int_{V_{K}} A(\kappa) \exp (i \kappa \bar{r}) \quad d V_{K}
$$


By integrating the scattering profile, the structure or electron density distribution of the scattering material can be obtained. The electron density distribution is not, however, uniquely defined by this integral. More subtle methods are necessary to obtain information about the electron density distribution.

Experimentally, the scattering intensity is measured not the amplitude. The intensity is the square of the amplitude and is equal to the product of the amplitude and its complex conjugate.

$$
I(\kappa)=A A^{*}=\int_{V_{1}} \int_{V_{2}} \rho\left(\bar{r}_{1}\right) \rho\left(\bar{r}_{2}\right) \exp \left(-i \kappa\left(\bar{r}_{1}-\bar{r}_{2}\right)\right) d V_{1} d V_{2}
$$

This integration can be performed in two steps. The first is the selfconvolution or autocorrelation of the electron density function.

$$
\begin{aligned}
\left\langle\rho(\bar{r})^{2}\right\rangle & =\int_{V} \rho\left(\bar{r}_{1}\right) \rho\left(\bar{r}_{2}\right) d V_{1} \\
\bar{r} & =\bar{r}_{1}-\bar{r}_{2}
\end{aligned}
$$

Substitution of equation (2.19) into equation (2.18) yields an intensity expression that is again a Fourier transform.

$$
I(\kappa)=\int_{V}<p(r)^{2}>\exp (-i k \bar{r}) d V
$$

The structure of the scattering material as defined by $\left\langle_{p}(r)^{2}\right\rangle$ can be obtained from the inverse Fourier transform of $I(k)$.

$$
<\rho(\bar{r})^{2}>\left(\frac{1}{2 \pi}\right)^{3} \quad \int I(k) \exp (i k \bar{r}) d k
$$


SAXS analyses can be simplified when the scattering material is statistically isotropic and when no long range order exists. For a fixed angle from the transmitted beam a statistically isotropic material will scatter at an equal intensity at any angle around the transmitted beam. In other words, the scattering will be in cones of equal intensity about the transmitted beam. A two phase system will scatter isotropically when the dispersed phase is randomly or symmetrically oriented with respect to the axis of the incident beam. For example, a dispersion of rods will scatter isotropically if the rods are either randomly oriented throughout the sample or if the axes of the rods are alligned parallel to the beam axis. When this restriction is valid it follows that the auto-correlated electron density function as well as the resultant scattering depend only on the magnitude of $r$. This is not true for the electron density function in real space. Based on this, Debye showed that the phase factor could be replaced by its average over all $r(23)$.

$$
\exp (-i \kappa \bar{r})=\frac{\sin k r}{k r}
$$

No long range order means that there is no correlation between two points that are widely separated. At large values of $r$ these electron densities should become independent and can be replaced by a mean value $\dot{\rho}$. At smaller values of $r$ the electron densities are still given by $p$. Structure is only defined by the electron density function in the region of small $r$ where $\rho$ deviates from $\bar{p}$. This is also the only region from which diffraction can originate. Therefore it is the 
fluctuations in electron density that are important rather than the electron density itself. The auto-correlation of the electron density function is then redefined as:

$$
\left\langle\rho(r)^{2}\right\rangle=\left\langle(\rho(r)-\bar{p})^{2}\right\rangle
$$

When a system scatters isotropically and when there is no long range order the simplifications expressed in equations (2.22) and (2.23) reduce the intensity expression to:

$$
I(\kappa)=\int<\rho^{2}(r)>4 \pi r^{2} \frac{\sin k r}{k r} d r
$$

\subsubsection{Particle Size}

One of the first applications of SAXS theory was the evaluation of particle size. Qualitatively, with a small particle of radius $d$, there will be alot of scattering centers (electrons) in the particle that are slightly less than $d$ apart. These electrons will diffract at an angle 20 where the $x$-ray path lengths differ by one wavelength (Fig. 2.6). Given a larger particle with a radius of $2 d$, there are likely to be a large number of scattering centers just less than $2 d$ apart. The angle at which first order diffraction occurs in this bigger particle is less than in the smaller particle. The preceding example demonstrates that for larger particles the scattering intensity will be shifted towards smaller scattering angles. Of course, from any size particle there will be interference from a distribution of interatomic distances and a diffuse scattering curve will result. The shape of this curve will 
change with particle size.

A single isolated particle with spherical symmetry will obey the restrictions leading to equation (2.24). The electron density outside the particle is taken as zero leading to:

$$
\left\langle\rho^{2}\right\rangle=\left\langle(\rho-0)^{2}\right\rangle=\rho^{2}
$$

Guinier replaced $r$, which defined the location between two scattering centers, with the radius of gyration (22). The radius of gyration, $\mathrm{Rg}$, is the root mean square distance of the scattering elements in the particle from the center of electron density and is a measure of particle size. For a spherical particle $\mathrm{Rg}$ is roughly $0.77 \mathrm{r}$ where $r$ is the radius of the sphere. Expanding the phase factor in a power series, truncating the higher order terms and substituting the result in equation (2.24) yield

$$
\begin{aligned}
I(k) & =(\rho V)^{2} \exp \left(-\frac{1}{3} \kappa^{2} R_{g}{ }^{2}\right) \\
\ln [I(\kappa)] & =2 \ln [\rho V]-\frac{1}{3} k^{2} R_{g}^{2}
\end{aligned}
$$

This is the well known Guinier approximation which is utilized by plotting the natural logarithm of intensity versus the scattering vector squared. The radius of gyration is obtained from the limiting slope as the scattering vector approaches zero. The Guinier approximation was derived above for a single particle but is valid for a dilute monodisperse system (sum of scattering from single particles). The higher order terms in the power series expansion cannot be neglected 
when the particles are anisotropic. Any deviation from spherical shape at constant $\mathrm{Rg}$ will involve larger distances and increase the higher order terms. As a result, estimates of $\mathrm{Rg}$ will be too large. Polydispersity has the same effect.

\subsubsection{Babinet Principle}

The Guinier approximation is not restricted to systems of isolated particles. As discussed before, electron density differences result in diffraction. A system with particles of electron density $\rho(1)$, dispersed in a matrix of electron density $\rho(2)$, can be thought of as a system of isolated particles of electron density $p(1)-p(2)$. In a two phase system, it is the difference in the electron densities that is important not their finite values. The scattered intensity is proportional to the square of the deviation from the average electron density. In a two phase system with sharp phase boundaries a mean square electron density variance can be computed from equation (2.23)

$$
\begin{gathered}
\left\langle\rho(r)^{2}\right\rangle=\left\langle(\rho(r)-\bar{p})^{2}\right\rangle \\
\bar{\rho}=\phi(1) \rho(1)+\phi(2) \rho(2) \\
\overline{\Delta O}^{2}=\phi(1) \phi(2)[p(1)-p(2)]^{2}
\end{gathered}
$$

where $\phi(1)$ and $\phi(2)$ are volume fractions. The mean square electron density variance is proportional to the electron density difference 'squared' indicating that the scattering contrast is independent of which phase is of higher electron density. This corresponds to the 
Babinet principle of reciprocity characteristic of many optical techniques. Equation (27) also predicts that for a given two phase system, the contrast is greatest when the volume fractions for the two phases are equal to 0.5 .

\subsubsection{Correlation Function}

In a spherically symmetric system, the electron density function can be pictured as changing only with the length of the vector between scattering centers where $r$ is a vector extending radially outward from a center of spherical symmetry. The correlation function is defined as the average mean square electron density variance for all points located a distance $r$ apart normalized by the mean square electron density variance over all interactions.

$$
\gamma\left(\bar{r}_{1}-\bar{r}_{2}\right)=\gamma(\bar{r})=\frac{\left\langle\Delta \rho\left(r_{1}\right) \Delta \rho\left(r_{2}\right)\right\rangle}{\overline{\Delta \rho}}-\frac{2}{2}
$$

The electron density function in equation (24) can be rewritten as the product of a constant mean square electron density variance and the correlation function which contains the spacial dependence of the electron density in the sample.

$$
I(\kappa)=\overline{\Delta 0}^{2} V \int_{0}^{\infty} r(r) r^{2} 4 \pi \frac{\sin k r}{k r} d r
$$

The correlation function is obtained by taking the inverse Fourier transform of equation (2.29).

$$
\gamma(r)=\frac{1}{2 \pi^{2} \sqrt{\Delta 0^{2}}} \int_{0}^{\infty} I(\kappa) \kappa^{2} \frac{\sin \kappa r}{\kappa r} d k
$$


The value of a correlation function is related to the probability that a rod of length $r$ has both ends in an area of equal electron density. The limiting values are one when $r=0$ and zero as $r$ approaches infinity. A maximum in the correlation function corresponds to some type of periodicity in the sample. The first maximum in the three-dimensional correlation function described above should correspond to the interdomain spacing obtained through Bragg's law.

When $r$ is set equal to zero, equation (2.30) reduces to

$$
Q=2 \pi^{2} \sqrt{\Delta \rho}{ }^{2}=\int_{0}^{\infty} I(\kappa) \kappa^{2} d k
$$

This integral is called the 'invariant' because it is not sensitive to the features of the structure or the changes in shape of the scattering profile due to these features. It is directly related to the mean square electron density variance of the sample and is important in many SAXS analyses.

The mathematics of the three-dimensional correlation function can be explained by considering a rod of length $r$ centered on one scattering center. At length $r$ the rod is rotated in all directions until the path traced by the unfixed end of the rod forms the surface of a sphere. At every position on this sphere the local electron density is accessed. The probability that both the scattering center and the end on the surface of the sphere are in regions of the same local electron density is calculated. This is done for all lengths of $r$ 
and for all scattering centers to yield the three-dimensional correlation function expressed by equation (30).

Analogously, a ray can extend in one direction from a scattering center and the electron density can be measured along the length of this ray. More rays can be drawn from the same point until electron density measurements along rays extending in all directions have been averaged. This is repeated for every scattering center. The result is a one-dimensional correlation function which is expressed mathematically by

$$
\gamma(x)=\frac{\int_{0}^{\infty} k^{2} I(k) \cos k x d k}{\int_{0}^{\infty} k^{2} I(k) d k}
$$

where the denominator is just $Q$, the invariant.

The two calculated correlation functions are usually similar. The position of the first maximum in the 1-D correlation function is usually shifted to slightly smaller distances. This is a result of the weighting differences involved in measuring electron densities along spherical surfaces versus that along a rod. One-dimensional correlational functions are used when the morphology consists of alternating layers of material as in a system with isotropically oriented bundles of stacked lamallae. In this system, the position of the first maximum would be a measure of the interlamellar spacing.

\subsubsection{Porod's Law}

In 1951, Porod derived an expression that indicates that internal 
surfaces or areas of sharp change in electron density are responsible for the final slope in a small angle intensity profile. For an ideal two phase system with sharp phase boundaries the intensity in the limit of high scattering vector is given by

$$
\lim _{k \rightarrow \infty} I_{p}(k)=\frac{k_{p}}{k^{4}} \text { or } \lim _{k \rightarrow \infty} I_{p}(k) k^{4}=k_{p}
$$

where $K p$ is a constant related to the surface to volume ratio of the phases (24-26).

In real systems the product $I(\kappa) \kappa^{4}$ may not reach a constant value. When the product $I(k) \kappa^{4}$ increases with $k$, the material shows positive deviations from Porod's law. These result from thermodynamic-scale density fluctuations (background scattering) and from the mixing or entrapment of units of one phase in the other phase often called 'phase mixing'. Negative deviations result from the presence of diffuse phase boundaries. Ruland proposed a modification to Porod's law which provides a means to characterize the diffuse boundary (27).

$$
\lim _{\kappa \rightarrow \infty} I_{\text {obs }}(\kappa)=I_{p}(\kappa) H^{2}(\kappa)+I_{B}
$$

In equation (2.34) the observed intensity is equal to the sum of the background intensity and the product of an ideal Porod's law intensity and a damping function, $H(k)$. There is no way at present to measure the intensity contribution due to phase mixing and this must be assumed negligible. The validity of this assumption for urethane systems has been discussed by Tyagi (28). 
Background intensity is the result of the atomic-scale electron density fluctuations that are of statistical thermodynamic origin. It arises because molecules are not stationary, but vibrate. Structural analysis from SAXS theory is based on fixed scattering centers and does not include background scattering. Various theoretical treatments to estimate background scattering have appeared and are reviewed by Koberstein (29). None of the treatments are fundamentally sound from first principles but all result in similar estimates of the background intensity. The background intensity increases only slightly with angle and can be considered constant in the small angle region. Subtraction of background does not change peak positions or slopes but is important for analyses that involve integration of the scattering profile and analysis of the tail region. In the tail region, background scattering is more intense than interference scattering and proper estimation of background is crucial for determination of interfacial thicknesses using Porod's law.

Removal of background intensity from the observed intensity results in the corrected intensity.

$$
I(K)=I_{C}(K)=I_{\text {obs }}(K)-I_{B}
$$

Equation (2.34) can be simplified to

$$
\lim _{k \rightarrow \infty} I(k)=I_{p}(k) H^{2}(k)=\frac{k p}{k^{4}} H^{2}(k)
$$

In order to determine a diffuse boundary thickness, the form of the electron density gradient across the diffuse boundary must be 
assumed. Expressions for both linear and sigmoidal gradients have been developed and are summarized by Koberstein (29). Through statistical thermodynamic arguments Helfand and Telgami have justified the existence of diffuse boundaries between phases in heterogeneous blends and segmented copolymers and have shown that the gradient in these diffuse boundaries should be sigmoidal in form $(40,41)$. For the nonequilibrium case, Koberstein has also noted that a system that undergoes Fickian diffusion will have a sigmoidal gradient (29). Therefore, the sigmoidal gradient model is believed to be more realistic. The smoothing function for the sigmoidal gradient model is Gaussian and is given by:

$$
H(\kappa)=\exp \left(-\frac{1}{2} \sigma^{2} \kappa^{2}\right)
$$

The value of $\sigma$ is related to the standard deviation of the Gaussian smoothing function and is a measure of the diffuse boundary thickness (Fig. 2.7). The diffuse boundary thickness assuming a linear gradient between phases would equal o 12 . Determination of the diffuse boundary width is of interest because of how it can influence mechanical properties (42).

\subsubsection{Degree of Phase Separation}

The electron density function was defined in equation (23) as the convoluted square of the difference between the local and mean electron densities.

$$
\left\langle\rho(r)^{2}\right\rangle=\left\langle(\rho(r)-\bar{\rho})^{2}\right\rangle
$$


It provides the contrast for $x$-ray diffraction. Conceptually, one can walk through a material along a given ray and continually measure the local electron density. In an ideal two phase system with sharp phase boundaries one may observe an electron density profile as depicted in Figure 2.8a. The difference between the measured electron density along the ray (solid line) and the mean electron density is a measure of scattering contrast as suggested by equation (23). Analogously, the area enclosed by the electron density profile and the mean value can be taken as a measure of contrast. Theoretically, the mean square variance in electron density for this system is given by:

$$
\overline{\Delta \rho}^{2}=\phi(1) \phi(2)[\rho(1)-\rho(2)]^{2}
$$

In real two phase systems, boundaries may not be sharp, the phases may not be pure (phase mixing), and thermal density fluctuations (background intensity) are present. These affect the electron density profile as depicted in Figure 2.8b. The background intensity is responsible for small fluctuations all along the electron density profile. The presence of diffuse boundaries is denoted by the nonvertical change in electron density between phases. The presence of phase mixing is denoted by decreased maximums and increased minimums in the electron density profile as the pure phases are contaminated with the other phase. Diffuse boundaries and phase mixing reduce the area enclosed between the measured electron density profile and the mean electron density and thus lower the scattering 
contrast. By subtracting the background intensity an electron density is obtained that reflects only the diffuse boundaries and phase mixing (Fig. 2.8c). Experimentally, the contrast factor (mean square electron density variance) for this electron density profile can be calculated from the invariant (equation 31 ).

$$
\overline{\Delta p}^{\prime}=\frac{1}{2 \pi^{2} i_{e}} \int_{0}^{\infty} I(k) k^{2} d k
$$

In the Porod's law analysis, the scattering contribution due to diffuse boundaries is evaluated. If this contribution is taken into account, an electron density profile that reflects only phase mixing is obtained (Fig. 2.8d). The mean square electron density variance for this electron density profile can be calculated from

$$
\overline{\Delta \varphi}^{2}{ }^{\prime \prime}=\frac{1}{2 \pi^{2} i_{e}} \int_{0}^{\infty} \frac{I(\kappa) \kappa^{2}}{H^{2}(\kappa)} d k
$$

where $H(k)$ is the smoothing function from the Porod's law analysis.

The mean square electron density variance for an ideal two phase system with sharp phase boundaries can be calculated from equation 2.27 using the electron densities of the pure phases and volume fractions of the phases. Experimentally, electron density variances can be determined for real two phase systems where phase mixing and diffuse boundaries possibly occur (equation 2.38) and for a real system in which the diffuse boundary contribution has been removed (equation 2.39). As seen in Figure 2.8 the contrast and therefore the mean square electron density variance is decreased by phase mixing and diffuse boundaries. This decrease in contrast can be used as a 
qualitative measure of the state and degree of phase separation.

The overall degree of phase separation is reflected in the ratio of the variance determined from the data to the calculated electron density variance for an ideal two phase system.

$$
\overline{\Delta \rho}^{2} / \overline{\Delta \rho}^{2}
$$

This ratio should in theory reach a value of one with complete phase separation (no boundary or phase mixing). The presence of diffuse boundary mixing, irrespective of phase mixing, is given by

$$
\overline{\Delta \rho}^{2 \prime \prime} / \overline{\Delta \rho}^{2}{ }^{\prime}-1
$$

involving the two experimentally determined electron density variances. This expression is zero for a system with sharp boundaries and increases with increasing boundary thickness. The presence of phase mixing, irrespective of the diffuseness of the boundaries, is given by

$$
\overline{\Delta \rho}^{2} / \overline{\Delta \rho}^{2 \prime}-1
$$

which again is zero when there is no phase mixing an increases as the extent of this type of mixing increases. As stated above, information obtained from these expressions is qualitative but can be valuable in accessing the degree and state of phase separation.

\subsection{SUMMARY}


A typical small angle scattering profile for a phased separated segmented urethane is shown in Figure 2.9. The intensity below the dotted line is background intensity and is subtracted from the data. The diffuse peak is interpreted in terms of Bragg's law to yield an average center-to-center spacing between domains. The initial portion of the scattering curve is evaluated in terms of the Guinier approximation. The slope of a plot of $\ln [I(k)]$ versus $k$ is related to the size of the scattering particles. The final portion of the scattering curve is evaluated in terms of Porod's law which results in a measure of the diffuseness of the phase boundaries. Integration of the scattering profile yields an interdomain spacing via the correlation function approach and mean square electron density variances. From the electron density variances various statements can be made as to the degree of phase separation, the amount of phase mixing, and the amount of boundary mixing. 


\section{CHAPTER THREE: EXPERIMENTAL}

\subsection{FOAM PREPARATION}

The conventional slabstock foams used in this investigation were produced in a homemade, discontinuous box foam machine. The box foam machine consists of a mixing chamber and a pasteboard box. The mixing chamber is a 16 inch diameter carbon steel baffled tank. It is equipped with an 8 inch inclined plane agitator that is driven by a 7.5 horsepower motor. The pasteboard box is a three foot cube with an open top face and is lined for free rise production.

The foams were prepared by adding the polyol, water and blowing agent, amine catalyst, and surfactant to the mixing chamber and mixing for 30 seconds at 900 revolutions per minute. The tin catalyst was then added and the mixture was mixed for an additional 30 seconds at the same speed. Agitation was stopped and the mixing chamber was degassed for 45 seconds. Isocyanate was then added and the contents were mixed for 5 seconds at $1200 \mathrm{rpm}$. The mixture was poured into the lined box and allowed to react. This procedure was used to produce all of the foams investigated.

After a foam had cured, the pasteboard box was torn away and the foam was trimmed. Four inches were taken off the top of each box 
foam and discarded, two inches were taken off the bottom and three inches off each side. Part of the remaining block was compression molded into a solid plaque by pressing at 250 tons of pressure and 204 $C$ for 10 minutes. The rest was cut into smaller pieces and subjected to various methods of analysis.

The formulation components used to produce these foams are summarized in Table 3.1. All components are commercial products and are routinely used in the manufacture of conventional slabstock foams. A series of six foams was produced using these components. In the first four foams the water level was varied from 2 to 5 parts by weight per hundred parts polyol ( $p p h$ ) while all other variables were held constant. In the fifth and sixth foams, the amount of tin catalyst was varied. In all formulations, the isocyanate index was 110. The formulations are summarized in Table 3.2. This series of foams has been given the label 31135-33-X. In this thesis, the 31135-33-X foams and plaques are often referred to as just foam $X$ or plaque $X$ where $X$ can be related to the formulations in Table 3.2.

Several other series of foams were made to study the affects of changes in other formulation variables. These foams are not the main thrust of the author's work but are included here for completeness. The affects of adding a blowing agent are studied in series 31857-37-X. Polyols of different molecular weights are used in the series $31857-40-X$ foams and in series $31857-45-X$ the TDI index is varied. These foams were made with the same formulation components 
as the $31135-33-X$ series only different components were varied. The formulations are summarized in Table 3.3.

\subsection{Methods of Analysis}

The changes in formulation variables resulted in noticable changes in the cell structure of the foams. These changes were documented using scanning electron microscopy. The mechanical properties of the foams were evaluated and contrasted with the properties of the compression molded plaques. The morphology of the foams and plaques was investigated using dynamic mechanical analysis, DSC, FTIR, TEM, WAXS, and SAXS. The details of these methods of analysis are presented below.

\section{2 .1 SEM}

Scanning electron microscopy was used to study the differences in cell structure between the various foams. Slices of foam 1 to 2 millimeters thick were cut from the blocks of foam along planes parallel and perpendicular to the blow direction. The foam slices were affixed to aluminum stubs by applying silver paint to the surface of the stub and gently pressing the foam slice in it. Care was taken to avoid contact between the silver paint and the upper surface of the foam. Approximately 200 angstoms of $\mathrm{Au} / \mathrm{Pt}$ was applied to the foams using a SPI Model 13131 sputter. Micrographs were taken on an ISI Super III- 
A scanning electron microscope operated in the secondary electron mode at a $15 \mathrm{kV}$ accelerating voltage.

\subsubsection{Mechanical Testing of Foams}

The foam samples used for mechanical property measurements were taken from four inch thick slices of foam cut from the middle of the slabs. In all testing, properties were evaluated along the blow or rise direction. Testing procedures specified in the ASTM standard D-3574-81 were used. These procedures are reviewed below as an aid to the reader in interpreting the results.

According to this standard, all foams tested should be at least seven days old. Prior to the tests the foams should be conditioned undeflected and undistorted for 12 hours at the temperature and humidity of the test. The recommended atmospheric conditions are a temperature of $23+2 \mathrm{C}$ and relative humidity of $50+5$ percent. Finally, before testing all foams should be compressed twice to between 75 and 80 percent of their thickness original thickness.

The density of a foam is determined by weighing a known volume of foam. The volume of foam should be not less than $1000 \mathrm{~mm}^{3}$ and should be from a portion free of defects and voids.

The tensile strength and percent elongation are measured on an Instron-type tensile tester using a dogbone-shaped foam sample. The foam sample is cut with a die to avoid nicks or ragged edges. The dimensions of the die and the rate of elongation are specified in the 
ASTM standard. The tensile strength is defined as the breaking force divided by the original cross-sectional area. Percent elongation is defined as the elongation at break minus the original length, normalized by dividing this sum by the original length and multiplying by one hundred.

Tear resistance is measured on an Instron-type tensile tester using a long foam block with a deep slit cut in the center of one end. The portions of the sample separated by the slit are mounted into the upper and lower jaws of the Instron such that when these jaws are moved apart the slit is pulled apart and the foam begins to tear at the base of the slit. The dimensions of the sample and the rate of elongation are specified in the ASTM standard. The tear strength is defined as the maximum force measured while the tear is propagating divided by the width of the sample.

The resilience of a foam is measured by dropping a steel ball on to the surface of a foam and measuring the rebound height. Resilience is defined as the rebound height divided by the drop height. The drop height, foam thickness, and size and weight of the steel ball are specified in the ASTM standard.

ASTM also specifies a test for measuring the compression set in polyurethane foams. In this test the preconditioning of the foams as described earlier is crucial. For the $90 \%$ CT test, the foam is compressed to $90^{\circ}$ of its original thickness and then placed in an oven at $70 \mathrm{C}$ and $5 \%$ relative humidity for 22 hours. At this time the foam 
is removed from the oven, released from the compressed state, and allowed 30 minutes to recover before the thickness is measured. The compression set is defined as the original thickness minus the final thickness, normalized by dividing the sum by the original thickness and multiplying by one hundred.

The most popular method of measuring compressive strength is the indentation force deflection test. In this test a square foam slice is compressed by a circular indentor that is smaller in diameter than the length of the square. This test mimmicks the demands placed on a foam in the mattress and upholstery industries more closely than a compression test in which the top plate covers the entire surface of the foam. In the test, the sample is compressed twice to between 75 and 80 percent of its original thickness and allowed five minutes to recover. Then the indentor is lowered at a fixed rate until the indention is $25 \%$ of the original foam thickness. The load is recorded after 1 minute. The indentor is lowered further until the indention is $65^{\circ}$ of the original foam thickness and the load is again recorded after 1 minute. On releasing the indentor, the load is again measured after pausing for 1 minute at $25^{\circ}$ indentation. These values are the $25 \%$ and $65 \%$ ILD values and the Return 25\% ILD value.

It is common practice in the foam industry to ratio the $65 \%$ ILD to the 25\% ILD and call this the modulus of the foam. For upholstery uses, the higher the modulus, the better. High modulus foams are soft to small deformations (low 25\% ILD) and firm to large deformations 
(high 65\% ILD).

It is also common to ratio the return $25 \%$ ILD value to the $25^{\circ}$ ILD value and call this the foam hysteresis. This can be confusing because when studying elastomers hysteresis is defined as the difference between the work in the loading and unloading cycles. The higher the hysteresis, the poorer the performance of the elastomer. As defined above, foam hysteresis is a measure of foam recovery after deflection and the higher the value the better the performance of the foam.

Dividing the $25 \%$ ILD by the foam density yields the 'guide factor'. The higher the guide factor the more cost efficient the foam because it has higher performance per pound. Of course, as defined above the guide factor does not incorporate many of the different aspects of foam performance.

The air flow test measures the ease with which air can pass through a cellular structure. The air flow through foams is influenced by many factors including cell size, distribution of cell sizes, and the extent of cell opening. In the ASTM test the air flow value represents the volume of air per second at standard temperature and pressure required to maintain a constant pressure differential of 125 $\mathrm{Pa}$ across a flexible foam specimen 50 by 50 by $25 \mathrm{~mm}$.

\subsubsection{Mechanical Testing of Plaques}


Instantaneous permanent set and hysteresis were measured for the six compression molded plaques from series 31135-33-X. An Instron Model 1122 tensile tester was used for the measurements. Dogbone samples were cut using a die. The samples were exposed to a 25 percent strain, unstrained to a point of zero stress, and then exposed to a higher strain of 50 percent. This cycle was repeated with higher incremental strains until the sample broke. The crosshead speed was $10 \mathrm{~mm} /$ minute in both directions. Instantaneous permanent set was calculated from the sample length at zero stress. Hysteresis was calculated from the difference between the areas under the loading and unloading stress-strain curves normalized by the area under the loading curve. Due to limited materials, the data presented later is based on the experimental results using a single sample of each material. The tests were repeated and comparable results were found for every plaque.

\subsubsection{Dynamic Mechanical Spectroscopy}

Dynamic mechanical spectroscopy was used to locate the soft segment glass transition, observe modulus-temperature behavior, and to look for the presence of other thermal transitions. Experiments for both the foams and plaques were performed using either a Rheometrics Model 605 Mechanical Spectrometer or a Rheometrics Model 7700 Dynamic Spectrometer. Foams were tested in a parallel plate mode using cylindrical samples. The sample was subjected to an oscillatory shear deformation at a frequency of 1 Hertz and at a strain of 
approximately 1 percent. The temperature range was from $-160 \mathrm{C}$ to 300 C. Data was collected at five degree intervals. Temperatures were maintained for two minutes before readings were taken.

The foam samples were cut with a high speed cork borer such that the cylinder axes were alligned with the blow direction of the foam. The foam cylinders were $25 \mathrm{~mm}$ in diameter and $25 \mathrm{~mm}$ in height. The foam cylinders were mounted on parallel plates with General Electric Silicone High Temperature Gasket Sealant. The sealant was allowed to dry for thirty minutes with the sample mounted in the instrument and slightly compressed.

\subsubsection{Differential Scanning Calorimetry}

DSC was performed on the series $31135-33-X$ foams to determine the soft segment glass transition temperatures, to note the temperature stability of the foams and to allow comparison of any other thermal transitions with dynamic mechanical data. The experiments were performed on a Mettler Model TA 3000. Samples were annealed in the DSC at $120 \mathrm{C}$ for 5 minutes to drive off moisture and then scanned from $-120 \mathrm{C}$ to $250 \mathrm{C}$ at the rate of $20 \mathrm{C}$ per minute.

\section{2 .6 FTIR}

FTIR was used to investigate the changes in concentration of the various chemical groups in the foams and plaques that are brought about by changes in formulation variables or the compression molding process. Infrared spectra were collected on a Digilab FTS-10 Fourier 
transform infrared spectrophotometer equipped with a germanium coated cesium iodide beamsplitter and a triglycine sulfate (TGS) detector. The spectra were collected using a Harrick TMP-220 internal reflection unit. A KRS-5 prism was used and the angle of incidence between the infrared radiation and the prism was 50 degrees. The spectra were collected at 8 wavenumbers resolution and 2048 scans were collected for signal averaging.

The foam samples for the ATR experiment were cut from a large piece of foam and were compressed against the KRS-5 prism using the standard clamps supplied with the ATR accessory.

Microtomed samples of the compression molded plaques were prepared using a Sorvall MT2-B Porter-Blum ultramicrotome fitted with a diamond knife. Cuts were made along the thickness of the filmshaped compressed plaques.

In addtion to all the series $31135-33-X$ foams and plaques, FTIR was used to study humid aging and to study the extract from a swollen foam. In the humid aging study, a sample of foam 4 was placed in an autoclave at 13 pounds of pressure, $121 \mathrm{C}$, and $100 \%$ relative humidity for 4.75 hours. In the extraction study, foams were extracted with DMF at room temperature overnight. The extracted DMF solution was filtered from the foams and then evaporated leaving just the extract.

\subsubsection{Transmission Electron Microscopy}


The foams and plaques were observed under a transmission electron microscope in hopes of observing a phase separated domain structure. A Joel $100 \mathrm{C}$ Analytical TEM was used operated at an accelerating voltag of $80 \mathrm{kV}$.

The foam samples were prepared by fitting small rectangles of foam into Beem capsules and imbedding the capsule with Dow epoxy resin DER 331 using a vacuum pump to remove entrapped air. The capsules were cured at $60 \mathrm{C}$ for eight hours. The cured foam and epoxy capsules and the compression molded plaques were sliced into sections approximately 120 nanometers thick using a Sorvall MT-2 ultramicrotome at -80 C. Sections were placed on 300 mesh copper grids for the TEM analysis.

\subsubsection{WAXS}

Wide angle $x$-ray patterns were obtained for the foams and plaques to note the presence of any sharp rings due to crystalline or paracrystalline ordering and to note changes in structure due to the compression molding process. The WAXS patterns were taken in a standard Statton camera under vacuum and at a sample-to-film distance of $8 \mathrm{~cm}$. The $x$-ray source was a Philips table-top $x$-ray generator PW 1720 equipped with a fine focus tube. The radiation was nickelfiltered $\mathrm{CuK}_{\alpha}$ with a wavelength of 0.15418 nanometers. The plaques were approximately $1 \mathrm{~mm}$ thick. Foam samples were cut approximately $10 \mathrm{~mm}$ thick and compressed to less than $2 \mathrm{~mm}$ for the WAXS runs. Exposure times were 10 to 20 hours depending on sample thickness, 
foam density, and hard segment content.

\subsubsection{Cold Fracture Studies}

A common characterization technique for filled elastomers, semicrystalline polymers, and thermoplastic elastomers is to cold fracture the material and observe the fracture surface under a microscope. The filler particles, crystalline regions, and hard segments will fracture differently than the matrix material often enabling one to observe the sizes of the particles and their distribution in the matrix. This technique was applied to the series 31135-33-X plaques. The plaques were immersed in liquid nitrogen (bp -196 C) for five minutes and then fractured. These samples were prepared for the SEM by mounting them on aluminum stubs that were designed for mounting fracture surfaces and applying 15 nanometers of $\mathrm{Au} / \mathrm{Pt}$ with a SPI Model 13131 sputter. The Au/Pt was applied 5 nanometers at a time to avoid overheating the surface.

The technique of ion milling was used in an attempt to enhance the surface features of the cold fracture surfaces. Due to a lack of success this work is not discussed in detail. A summary of this work is presented in appendix A1.

\subsubsection{Swelling}

Swelling and extraction studies were used to measure the degree of gel formation (sol fraction) and the molecular weight between crosslinks. The affinity of different solvents for the polyurethane 
foams was determined by measuring the increase in length of a rod of foam after swelling in the solvent for 5 minutes. Longer swelling times were not necessary because the porous nature of the foam and thinness of the struts allowed equilibrium conditions to be reached almost immediately. All studies were at room temperature.

The sol fraction of the 31135-33 foams in two of the above solvents, DMF and THF, were determined and the sol fraction of the corresponding plaques were determined in DMF. Samples were immersed in the solvent for 100 hours before drying under a vacuum and measuring sol fraction. The sol fractions of the foams after 1 hour of exposure to solvent was already equal to the 100 hour value.

Equilibrium swelling data was obtained for the plaques of series 31135-33 in DMF.

\subsubsection{SAXS}

A major portion of the author's work utilizes the technique of small angle $x$-ray scattering. SAXS can be used to detect the presence of a phase separated morphology and to characterize the various features of the domain texture. The theory that serves as the basis for the analysis of the SAXS results was presented in Chapter 2. Data collection is discussed below.

Data were taken for the $31135-33-X$ series of foams and plaques and for the foams of the blowing agent, polyol molecular weight, and TDI index studies. Most of the data was obtained on the ORNL 
10-Meter SAXS camera. This instrument features a rotating anode $x$ ray source, crystal monochromatization of the incident beam, pinhole collimation, and a two-dimensional position-sensitive detector (43). A copper anode was used and the incident beam was monochromized to CuKa radiation. Details about data collection with the ORNL 10-Meter SAXS camera are given in reference 44 .

Complimentary slit-smeared data was obtained on a standard Kratky camera (45). The Kratky camera was equipped with a step scanning device interfaced with a computer. The $x$-ray source was Siemens AGF Cu 4ST tube powered by a General Electric XRD-6 generator. Again, the radiation was CuKa and a nickel filter was used to monochromize the incident beam. Data collection on a Kratky camera is discussed in more detail by Tyagi (28).

Samples were prepared as in the WAXS experiments. The compression molded plaques were used 'as molded' at thickness of about $1 \mathrm{~mm}$. Foam samples approximately $10 \mathrm{~mm}$ thick were compressed along the blow direction to about $6 \mathrm{~mm}$ for these experiments. 


\section{CHAPTER FOUR: RESULTS AND DISCUSSION}

\subsection{Morphological Considerations}

In the manufacture of a polyurethane foam, diisocyanates react with the active hydrogens of water and hydroxyl-terminated polyethers. The isocyanate-water reaction blows the foam and chain extends the hard segment. The isocyanate-hydroxyl reaction joins the trifunctional polyols and gels the foam. Catalysts are used to balance the instability of a rising foam (blowing reaction) with increased viscosity (gelling reaction) to produce a stable foam. One question that remains unanswered is how does the balance of reactions that is necessary in the formative stages of the foam effect the morphology of the final product? In particular, how does it effect chain extension, network formation, and phase separation?

In order to discuss the effect of the foam forming reactions on morphology, an expected or ideal morphology is needed. Based on the stoichiometry of the formulation ingredients for series $31135-33-X$ the following morphology is developed.

The $3000 \mathrm{MW}$ PPO polyol is assumed to have on average three equal length arms. The PPO repeat unit is shown in Figure 4.1a. Arms from two different polyols are joined by a chain-extended hard 
segment region. The repeat unit for the hard segment is shown in Figure 4.1b. Assuming complete reaction of the polyols and water with TDI and assuming the ten percent excess TDI added to the formulations is not involved, average degrees of chain extension were calculated. For foam 1, (2 pph water) the average chain between polyol junction points consists of two PPO arms 17.2 repeat units long joined by a hard segment consisting of 3.2 aromatic rings. For foam 4, (5 pph water) the soft segment arms are the same length and the hard segment consists of 6.5 aromatic rings. The hard segment to soft segment linkages are urethane linkages and the linkages between aromatic rings are disubstituted ureas. The structure of these chains is shown in Figure 4.2.

There are two soft segment arms between each hard segment or roughly 34 repeat units. If the soft segment arms were in the fully extended planar zig-zag conformation, they would be roughly 12 nanometers long ( 0.36 nanometers per repeat unit). Using chain statistics and the freely rotating chain model (49), the root mean square end-to-end distane of these arms in the material is roughly 3 nanometers. In the foams, the chains are probably extended above the statistical value by the presence of phase separated domains.

Hard segment dimensions for this hypothetical foam morphology are difficult to define because of the asymmetry of the polyurea repeat unit. The repeat unit can be traversed in as little as six bonds. Based on tabulated bond lengths and assuming every bond angle is 109 
degrees a rough measure of a fully extended polyurea repeat unit is 0.7 nanometers. This is about twice the length of the three backbone atom polyether repeat unit. Based on this, the average fully-extended hard segment lengths will vary from 2.2 nanometers for foam 0.1 to 4.5 nanometers for foam 4 . These hypothetical average dimensions for foams 1 through 6 are summarized in Table 4.1. In Table 4.2, the weight and volume fractions of hard segment for this series of foams and plaques are presented.

The soft segment is comprised of atactic PPO and cannot crystallize. Pure PPO has a glass transition of -75 C (46). If complete phase separation occured, the soft segment would be the continuous phase comprising roughly from 72 to 83 volume percent for this series of foams.

The hard segment is derived from the reaction of water with an 80:20 mixture of 2,4- and 2,6-TDI. It is assumed to be amorphous because of the asymmetry of the 2,4-TDI. The glass transition was reported by Tyagi to be above $200 \mathrm{C}$, because it was not observed before the onset of degradation in an urethane elastomer with similar hard segment and a PTMO soft segment (28). Paik Sung and coworkers reported that a polyurea of 2,4-TDI extended with ethylenediamine had a glass transition temperature of 203 C (47). They also reported that 2,6-TDI extended with butanediol could crystallize (48). With an 80:20 mixture of isomers and such short sequence lengths, hard segment crystallization is not anticipated but, 
as in MDI-based urethanes, the presence of hydrogen bonding may lead to some form of ordered packing (64). The hard segment content varies from 17 to 28 volume percent in the $31135-33-X$ series foams and plaques.

\subsection{Macroscopic Structure}

The cell structure of the foams was examined using scanning electron microscopy. Low and high magnification micrographs were taken of slices of foam cut parallel and perpendicular to the blow direction. These are shown for foams 1 through 6 of series $31135-33-X$ in Figures 4.3 through 4.8.

The micrographs of foam 1 (Fig. 4.3) reveal a distinct cell structure. Looking down the blow axis the cells appear spherical with diameters of roughly $1.2 \mathrm{~mm}$. Looking perpendicular to the blow axis the cells are ellipsoidal with the major axis of the ellipsoid alligned with the blow axis. The major and minor axes of these ellipsiods average roughly 1.5 and 1.2 millimeters, respectively. The sizes are fairly uniform with a standard deviation in sizes of less than twenty percent.

The cells are full of windows with diameters roughly one fourth the cell diameter. About $25^{\circ}$ of the windows are not broken and another 25\% are broken but still predominately intact. In an ideal formulation, 
the cell windows rupture and recede into the struts providing sturdier struts and improving air flow. Most of the windows are pentagonal.

The cell struts are very thick and this makes possible the observation of the distinct cells that existed as bubbles during foam rise. Windows were formed by the intersection of two expanding gas bubbles. The material that filled the gaps between three or more gas bubbles formed struts. In foam 1, both three and four sided struts are present.

In foams 1 through 4 the water level was systematically increased while the tin and amine catalyst levels were held constant. Addition of water will proportionally increase the amount of carbon dioxide released (blowing), increase the blowing reaction rate relative to the gelling reaction rate, and increase the temperature that the interior of the rising foam reaches.

Comparison of Figures 4.3 through 4.6 reveals that as the water level is increased the cells become slightly smaller and are proportionally more elongated in the blow direction. The cell struts become much thinner and the foam becomes more open (less unruptured windows present). The cell windows remain mostly pentagonal and the number of four sided struts decreases leaving predominately three sided struts.

The slight reduction in cell sizes occured when the volume of carbon dioxide released (water content) was increased by roughly 50 , 
100, and 150 percent. This indicates that more nucleation occured and that nucleation was roughly proportional to isocyanate or water content. In foam production it is believed that the nucleation centers are small gas pockets formed when dissolved gas is forced out of solution during the agitation process (1). These foams were made by degassing the mixed formulation components, adding the required amount of isocyanate, and mixing for 5 seconds. Therefore nucleation would be proportional to both the dissolved gas in the isocyanate and the amount of isocyanate that can react with water during the 5 second mixing period. When the water and isocyanate levels are higher, kinetically more carbon dioxide will be produced per part water based on an increased number of collisions. The result would be a higher nucleation rate and smaller cell sizes. The above nucleation mechanism is pure speculation but does agree with the manufacturing process and account for the decreasing cell size with increased water content.

The increased elongation of the cells with increasing water content is a result of the increased amount of blowing and the constrained sides of the box foam machine. (It would be interesting to see how the elongation of the cells corresponded with the rise heights of the foams. Maybe the volume of formulation components could be changed so that this series of foams could be reproduced with the same rise height. In this way another variable-asymmetry of the cells-could be studied.) The thinner struts arise from the higher nucleation density and the larger released gas volume. The improved cell opening can be 
attributed to many factors including the higher ratio of blowing to gelling reactions and the higher temperatures in the rising foam.

Foams 1 and 6 contain the same water content and differ only in tin catalyst concentration. The tin catalyst (gelling catalyst) concentration was decreased in foam 6 to produce an optimal foam. The cell structures in foams 1 and 6 (Figs. 4.3,4.8) are very similar. The decrease in gelling catalyst did not change the size or elongation of the cells significantly, but did improve the cell opening.

The tin catalyst concentration in foam 3 was increased slightly to yield an optimum foam, foam 5 . No significant differences in cell size, elongation, or degree of cell opening is evident in the micrographs of these foams (Figs. 4.5,4.7).

The scanning electron microscope was used to study the foams in the blowing agent, polyol MW, and TDI-index series. These micrographs are not shown here. In the blowing agent study foams were formulated with and without a blowing agent such that their bulk densities would be similar. The foam manufactured with the blowing agent is more open and has slightly smaller cells with thinner struts.

The polyol MW study consisted of foams made with 2000 and 3000 MW PPO triols and the same water content. The $2000 \mathrm{MW}$ polyol foam has larger, less elongated cells with thicker struts.

The foams in the TDI-index study were formulated at 4 pph water and with indices of 95, 105, 115, and 125. At low isocyanate index 
the foam has, for the most part, fully opened but many windows are still present. As the TDI-index increases, the foams become less and less open. Foam 31857-45-3 appears to have collapsed.

The foams in these supplemental studies were formulated closely to the foams of the main series, 31135-33. However, foams in these supplemental series that should be very similar in appearance to foams in the main series appear different. In particular, foam 31857-37-1 should resemble foam 31135-33-4 but has larger and less elongated cells. Foam $31857-40-2$ is much less open than foams $31135-33-3$ and 33-5, though this could result from different tin catalyst levels. Finally, foams $31857-45-2$ and $45-3$ should resemble foams $31135-33-3$ and 33-5 but again they have larger cell sizes and are less open. Mechanical property measurements also showed these differences.

The cell structures within each series are consistent and reflect the changes resulting from formulation changes. The similarity between the foams in pairs 1,6 and 3,5 illustrate this. Comparisons between the different series are not valid possibly due to changes in atmospheric conditions during manufacture of the different series or the use of raw materials from different lots.

\subsection{Mechanical Properties}

In general, raising the water level in a foam formulation results in 
a lower density foam with a more open cell structure and finer struts. The increased water level also increases the hard segment content in the foam. The hard segment is believed to form segregated domains that act both as filler and crosslinks $(1,2)$. In a solid urethane elastomer the presence of phase separated domains increases the modulus and ultimate strength as well as the hysteresis. The increase in hysteresis is thought to arise from deformation of the hard segment (50) .

The mechanical properties for the series $31135-33$ foams are summarized in Table 4.3. The changes in properties with increasing water content agree with those generally observed for water-blown flexible foams $(1,4,5,6)$.

The tensile strength, percent elongation, and tear resistance improve with increasing hard segment content. The resilience, compression set, and hysteresis worsen with increasing hard segment content. The 25\% ILD values decrease with hard segment content. When these values are normalized by foam density to yield guide factors, the guide factors increase with hard segment content. The air flow increases by a factor of three from the most dense foam to the least dense foam. Finally, the foam modulus, defined as the ratio of the $65^{\circ}$ ILD to the $25 \%$ ILD, remains fairly constant throughout the series of foams considering the very large changes in foam density.

The mechanical properties for the blowing agent, polyol MW, and TDI-index studies are presented in Table 4.4. The two foams in the 
blowing agent study have roughly the same density. This allows a comparison of foams with different hard segment contents and similar cell structures. From the SEM micrographs the cell structure appears very similar in the two foams. The foam manufactured with freon as a blowing agent is more open and has slightly smaller cells with thinner struts. The difference in openess is reinforced by a high air flow measurement.

The tensile strength and tear resistance are higher in the foam with higher hard segment content. The percent elongation is about the same for both foams indicating that cell structure may be more important than material strength in this test. Resilience and hysteresis improve as the hard segment content is lowered. Finally, use of the blowing agent significantly decreased both the 25\% ILD and the $65^{\circ}$ ILD.

In the polyol study, the polyol MW was varied while the water level was held constant. The lower MW polyol had a lower equivalent weight and therefore introduced more reactive hydroxyls into the foam formulation. This would decrease the hard segment length and could reduce the amount of phase separation.

The tensile strength, 25\% ILD, and $65^{\circ}$ ILD are all significantly higher in the foam with the higher MW polyol possibly indicating a higher degree of phase separation.

In the manufacture of slabstock foam, the TDI-index is often 
raised to increase the stiffness of the foam. Increasing the stiffness in this manner often results in poorer properties such as tensile strength, resilience, and hysteresis (6). These trends were observed in the TDI-index study. The values reported for foam 31135-45-3 reflect the partial collapse of the foam during production.

The compression molded plaques for series $31135-33$ were subjected to testing involving cycles of increasing strain from which hysteresis and instantaneous permanent set measurements were made. Hysteresis, in this test, is defined as the difference between the work in the loading and unloading cycles divided by the work on loading and multiplied by one hundred. In urethane elastomers, a hysteresis of fifty percent is not unusual. It is believed to result from disruption of the hard segments domains. It has also been related to the degree of interconnection between hard domains (60). Polyol molecular weight, hard segment molecular weight, and hard segment content strongly influence hysteresis behavior.

The hysteresis behavior for the $31135-33$ series of plaques is shown in Figures 4.9 and 4.10. All samples reach a level of constant hysteresis within three cycles ( $70^{\circ}$ elongation). The hysteresis increases very systematically with increasing hard segment and is typical of conventional urethane elastomers.

Instantaneous permanent set, as defined by this test, is a measure of how much deformation remains in a material that has had only the time required by the unloading cycle to recover. In the plaques, 
relaxations in the soft segment should occur quickly due to its low glass transition. Therefore, instantaneous permanent set is again a measure of hard segment deformation. The results are presented in Figures 4.11 and 4.12. The expected behavior is observed.

The instantaneous permanent set is shown in Figure 4.13 for a urethane-urea elastomer with a PTMO soft segment and TDI-based hard segment at a hard segment content of 31 weight percent (28). The hard segment repeat unit is the same as that in the foam but was synthesized using a novel technique based on the degradation of tertiary alcohols. At low elongations the urethane urea elastomer behaves very much like the compression molded plaques. The notable difference is that the compression molded plaques fail at strains of less than 150 percent whereas the urethane urea elastomer does not fail until exposed to a strain of 500 percent. One explanation for this is that PTMO can crystallize and strain-harden the elastomer whereas the PPO soft segment of the plaques cannot crystallize. A more probable explanation is the presence of allophanate and biuret crosslinks in the plaques which can lower the molecular weight between crosslinks and thus reduce the elongation at break. This could be clarified by comparing the moduli of the plaques and elastomer. Degradation as a result of compression molding could also decrease ultimate properties.

\subsection{Thermal Characterization}


The temperature behavior of series $31135-33$ foams and plaques was investigated using dynamic mechanical spectroscopy (DMS) and differential scanning calorimetry (DSC). The results of the DMS study were summarized by Spell et al (81) and are presented in appendix A2. Very briefly, the DMS spectra for the foams and plaques are similar to those for relatively soft ideal elastomers (Fig. 4.14). The storage modulus curves each reveal a sharp transition from the glassy state to the rubbery state followed by a long, flat rubbery plateau that extends above $200 \mathrm{C}$. The loss modulus curves in three of the six foams reveal a small and unexpected transition around $100 \mathrm{C}$. When the spectrum is rerun immediately after passing through the transition and without exposing the sample to the atmosphere, the transition does not occur and the modulus is slightly higher. The modulus also increases after thermal treatment in a nitrogen atmosphere. The loss transition and modulus change may be the result of the loss of adsorbed moisture. If this is confirmed it may be related to humid-aged compression set, which is a major problem in the flexible foam industry.

Comparison of the DMS spectra of the foams and plaques shows that the compression molding process slightly decreases the soft segment $\mathrm{Tg}$, further flattens the rubbery plateau, and extends the plateau to a higher temperature. This is evidence that the phase separation is enhanced or hard segment ordering is improved by the compression molding process. 
Increasing the water content does not affect the soft segment $\mathrm{Tg}$, increases the high temperature stability, and increases the modulus as evidenced in spectra normalized to the glassy state $\left(2 * 10^{10}\right.$ dynes $/ \mathrm{cm}^{2}$ ). The results are summarized in Table 4.5. The lack of change in soft segment $\mathrm{Tg}$ is evidence that a similar degree of phase separation exists in all samples.

Optimization of the tin catalyst levels increases the room temperature storage modulus, but no other significant changes were noted.

To the author's knowledge, there are no other DMS studies of flexible polyurethane foams in the literature. This technique, however, is commonly used to characterize urethane elastomers with butanediol (BD) extended MDI and TDI hard segments (51-54). In these systems, the soft segment $\mathrm{Tg}$ is observed to decrease with increased soft segment molecular weight and increase with increased hard segment content. The magnitude of these changes are brought out in the data of Seefried and coworkers $(52,53)$. In a polycaprolactone/MDI-BD system a change in soft segment MW from 530 to 2100 decreased the $\mathrm{Tg}$ by $70 \mathrm{C}$. Increasing the weight fraction of hard segment from $42 \%$ to $65 \%$ raised the $\mathrm{Tg}$ by $40 \mathrm{C}$.

A plot of this data is shown in Figure 4.15. Increasing the hard segment content not only raises the $\mathrm{Tg}$, it also reduces the sharpness of the transition and increases the modulus in the rubbery plateau. The rubbery plateaus in Figure 4.15 are typical of segmented urethane 
elastomers with MDI-BD hard segments. The plateaus have negative slopes and the modulus drops off sharply at from $80 \mathrm{C}$ to $150 \mathrm{C}$ depending on hard segment content. In comparison, the foams have superior elastomeric properties as evidenced by sharp glass transitions, rubbery modulii that are independent of temperature (flat rubbery plateau), and have thermal stability to well above $200 \mathrm{C}$.

In DSC studies, Paik Sung and coworkers observed the soft segment Tg's of elastomers with compositions more closely resembling polyurethane foams (47). These elastomers were polyurethane-ureas and consisted of a linear PTMO soft segment and a 2,4-TDIethylenediamine hard segment. The soft segment Tg's were lower than in PTMO/MDI-BD elastomers (55) and independent of hard segment content for a PTMO MW of 2000 indicating that phase separation improved when urethane linkages were replaced by urea linkages.

Recently, Tyagi used the technique of DMS to characterize MDIand TDI-based polyurethane-ureas (28). These polymers have PTMO soft segments and urethane-urea hard segments analogous to those in water-blown polyurethane foams (i.e. hard segments of just the urea linked aromatic isocyanate structures, no aliphatic character). In the MDI systems with a soft segment based on 2000 MW PTMO diols, the soft segment $\mathrm{Tg}$ is roughly $25 \mathrm{C}$ lower than in analogous $\mathrm{BD}$-extended systems and is independent of hard segment content. With a TDIbased hard segment the $\mathrm{Tg}$ is depressed further. Decreasing the soft segment molecular weight increases the soft segment $\mathrm{Tg}$. The 
influence of hard segment concentration at lower MW has not been determined. The rubbery plateaus for these urethane ureas are flat and extend above $200 \mathrm{C}$. The storage and loss spectra for these urethane ureas are contrasted with a typical urethane elastomer (MDI$B D$ ) in Figure 4.16.

The DMS work of Tyagi and Sung shows that urea linkages lead to a higher degree of phase separation and promote higher temperature stability than urethane linkages. In elastomers with urea linked hard segments, the soft segment $\mathrm{Tg}$ is independent of hard segment content.

The DMS spectra for the foams and the TDI-based polyurethaneurea are very similar. This indicates that despite the possibilities for a much more complex morphology in the foams their behavior is governed by the same forces that determine the DMS behavior in a system of linear segmented molecules synthesized under more controlled conditions .

Complimentary DSC data was collected for the foams of series 31135-33. The data shows glass transition values that are slightly lower than those from the DMS spectra and decrease slightly with increasing water content. On the first temperature scan of each foam a broad, low energy endotherm occurs over a temperature range of from $20 \mathrm{C}$ to $120 \mathrm{C}$, peaking about $80 \mathrm{C}$. If the samples are immediately rerun or if fresh samples are annealed at $120 \mathrm{C}$ for 5 minutes and then run, the endotherm is not observed. This 
endotherm is thought to be caused by the release of adsorbed moisture and to correspond with the energy loss transitions observed in the DMS study. No other peaks or transitions are observed before the onset of degradation. As in the DMS study, the onset of degradation begins at higher temperatures when the hard segment content is higher.

The DSC data supports the findings of the DMS study but in light of work by other researchers it cannot be definitely concluded that the transition observed at $80 \mathrm{C}$ on first scans is due to adsorbed moisture. In untreated samples of PTMO/MDI-BD elastomers, Cooper and coworkers $(55,56)$ observed three endotherms associated with the hard segment occuring at temperatures of 60-80 C, 120-190 C, and above $200 \mathrm{C}$. These were associated with the disruption of domains with limited short-range order, the disruption of domains with improved short-range order, and the melting of domains with microcrystalline ordering, respectively. Cooper and coworkers were able to show that by annealing these elastomers the hard segment ordering could be improved as evidenced by the shifting of these endotherms to higher temperatures.

The fact that the 60-80 C endotherm can be shifted up to the 120-190 endotherm (and not disappear above $100 \mathrm{C}$ ) indicates that in these MDI-BD systems this endotherm is not due to adsorbed moisture. In elastomers with 2,4-TDI-ethylenediamine hard segments an $80 \mathrm{C}$ endotherm has been observed (47). It, like in the foams only 
appeared on the first scan. More recently, Cooper and coworkers (57) showed the affect of room temperature aging of the annealed samples on the three hard segment endotherms in PTMO/MDI-BD elastomers. Samples in which the DSC analysis was run immediately after annealing did not show an endotherm in the 60-80 degree range. Upon aging the endotherm reappeared. In these unaged samples the soft segment $\mathrm{Tg}$ was higher than in the control sample indicating that annealing had decreased the degree of phase separation. A decrease in phase separation will increase the modulus of a thermoplastic elastomer. This could account for the increase in modulus observed on the DMS spectra of the foams that were rerun after passing through the 60-80 C transition.

\subsection{FTIR Study}

IR spectroscopy was used to characterize the foams and plaques of series 31135-33. The preliminary findings of this study were summarized by Harthcock and are presented in appendix A3. The IR spectra show the expected changes in chemical composition with increasing water content. The spectra also reveal the presence of unreacted isocyanate in the foams. The amount of unreacted isocyanate increases with water content. The compression molding process decreases the amount of unreacted isocyanate. Changes in the tin catalyst level result in only minor changes in the IR spectra and 
do not alter the relative amounts of urethane and urea linkages.

IR spectroscopy has been used to study the degree of phase separation in polyurethane-urea elastomers $(59,61-63)$. In these systems the urea carbonyls were completely hydrogen bonded indicating a high degree of phase separation. The free and hydrogen bonded urethane carbonyl absorptions were used to characterize the mixing at domain interfaces. Studies of model systems were required to sort out the peaks in the carbonyl absorption region. In these studies, model urea compounds had complete hydrogen bonding of all the urea carbonyl and $\mathrm{N}-\mathrm{H}$ groups. Sung concluded that a single carbonyl must hydrogen bond with two $\mathrm{N}-\mathrm{H}$ groups (59). This interpretation agrees with the non-planar three dimensional hydrogen bonding configuration proposed by Bonart based on $x$-ray investigations (64).

Peak assignments in the carbonyl region are summarized in Table 2 of appendix A3. The peaks for hydrogen bonded urethane carbonyls and free urea carbonyls overlap. Knutson and Lyman (61) concluded that there was no free urea carbonyl absorption in their systems based on the absence of either shifting or broadening of the $\mathrm{N}-\mathrm{H}$ stretch at 3320 wavenumbers relative to peaks in model systems. This allowed interpretation of the carbonyl region in terms of three absorptions: hydrogen bonded urea; hydrogen bonded urethane; and free urethane.

If the urea phase is relatively pure in the foams and plaques the changes observed in the IR spectra can be interpreted as changes in 
the relative amounts of free and hydrogen bonded urethane carbonyls. Based on this, as the water content is increased, more urethane carbonyls are hydrogen bonded. This could be the result of smaller interfacial regions or larger domains. Assuming constant urea purity, the compression molding process decreases the amount of hydrogen bonded carbonyls. This could result from deformation of the hard segments during compression. Again, this interpretation is dependent on the lack of an urea $\mathrm{N}-\mathrm{H}$ shift in the foams relative to pure urea.

IR spectroscopy was also used to study humid-aging and solvent extraction. The humid-aged foam sample has a lower unreacted isocyanate content than the control foam. This indicates that the high temperature and pressure used to age the sample increased the diffusion rate of moisture through the polymer such that isocyanate that had not reacted for a month at room temperature reacted within five hours.

The purpose of the extraction experiment was to learn which species were not fully incorporated into the foam network. Under mild extraction conditions, where degradation should not have been a problem, an extract was collected and analyzed. The extract was predominately urea. Methyl groups, possibly from the surfactant, were also present. The results of the extraction study are discussed in more detail later. 
Transmission electron micrographs for foams 1 through 4 are shown in Figures $4.17 \mathrm{a}-\mathrm{d}$. The micrograph for foam 1, the foam with the lowest water content, has a diffuse grainy texture on the 100 nanometer level (Fig. 4.17a). This diffuse grainy texture is not visible in the adjacent epoxy matrix indicating that it is real. The weak contrast, different levels of contrast, and variation in grain sizes indicate that the contrast probably originates from features much smaller than the 120 nanometer thickness of the specimen. This observation agrees with the fact that the compression molded plaque of this foam is transparent. Again, these diffuse grains vary in size and contrast, and most importantly are randomly dispersed.

The micrograph for foam 2 is shown in Figure $4.17 \mathrm{~b}$. The increase in hard segment content resulted in improved contrast and the appearance of larger hard segment aggregations. In the micrograph there are solid white areas that presumably result from regions of nearly pure soft segment, light grey areas, and darker grey blotches. The light grey areas result from exposure of the transmitted beam to an electron density between that of the white and dark grey regions. This electron density could result from either a mixed morphology or transmittance of the beam through relatively pure regions of both the hard and soft phases. (Some of the grey bands result from variations in sample thickness.) The darker grey blotches are the regions with the highest hard segment density. The hard segment dense regions 
are roughly 100 nanometers in diameter and are randomly dispersed (meaning some are isolated and some are agglomerated into 300 plus nanometer groups). The hard segment dense regions are surrounded by lighter grey regions giving the impression the dispersion of hard segments is semicontinuous.

The micrographs for foams 3 and 4 are shown in Figure 4.17, parts $c$ and $d$. The size of the hard segment dense regions increases with water content. For foam 4 these regions are roughly 300 nanometers in diameter. This agrees with the observation that opacity increases with water content in the corresponding plaques. The contrast improves a little as the hard segment content is increased possibly due to improved phase separation. The presence of random agglomerations and the semicontinuous nature of the hard segments are similar to that observed in foam 2 .

Micrographs for compression molded plaques 3 and 4 are not shown here but were very similar to those of foams 3 and 4 , respectively.

There is little published literature on TEM studies of polyurethane foams or polyurethane-urea elastomers. TEM hase been used by a number of authors to study the morphology of polyurethane elastomers with crystallizable MDI-BD hard segments (65-69). Depending on type of soft segment, hard segment content, film preparation technique, and thermal history the morphology of these systems varied from a highly phase separated system with 10 nanometer wide semicontinuous domains (69) to system with discrete spherulites that were easily 
visible under an optical microscope (65) to a system with with a semicontinuous fibrillar network of fibrills 60 nanometers wide (65). Wilkes and Chang propsosed a spherulitic model for an urethane-urea with an 'amorphous' hard segment based on rheo-optical studies (70). Kollmeier et al $(14,15)$ studied the sequence of reactions in foam making with IR spectroscopy. When the reacting foam-mix reached about $90 \mathrm{C}$, the urea carbonyl absorption shifted and the clear foammix turned opaque. Through IR studies on model compounds it was concluded that the shift in carbonyl absorption was the result of the precipitation of a macroscopic urea phase.

Based on the above investigations, the formation of some type of macrostructure in the foams cannot be taken as a surprise. This subject is discussed further after the presentation of wide and small angle $x$-ray diffraction data.

\subsection{SEM}

Scanning electron microscopy provided additional evidence of a two phase morphology with randomly dispersed hard segment agglomerations 1000 to 300 nanometers in size. The SEM micrographs of the cold fracture surfaces of plaques 1 through 4 are shown in Figures 4.18 and 4.21. The fracture surface of plaque 1 is smooth agreeing with the TEM micrograph of foam 1 which depicts no observable microstructure on the 100 nanometer level. The SEM 
micrographs for plaques 2,3 , and 4 show increasing surface texture with increasing hard segment content. The texture of plaque 2 resembles that of a fine grade of sandpaper and in plaques 3 and 4 the grain becomes much coarser. In plaques 3 and 4 discrete 100-300 nanometer protuberences are visible. The random distribution of the protuberences and at times the random continuity between them are very much like that observed through TEM.

The technique of ion milling was used in an attempt to enhance the surface texture of the cold fracture surfaces. Success was marginal and details of the study are in appendix $A 1$. Plaques 5 and 6 were used for this study. Plaques 5 and 6 have the same water content as plaques 3 and 1 , respectively. In plaque 5 the ion milling process appeared to at first enhance the surface contrast and then, on further milling, to change the features of the surface (Fig. 4.22). This behavior was attributed to at first the slow wearing away of the hard segment relative to soft segment enhancing the surface features. Then, as the hard segment groupings became more exposed to the ion beam they began to wear away and change the features of the surface.

Plaque 6 , had the low hard segment content of plaque 1 , and had a smooth cold fracture surface. When this surface was ion milled it 'melted' into a smooth brainlike texture with features 200 nanometers wide (Fig. 4.23). The smoothness of the brainy texture was attributed to the absence of large hard domain regions beneath the surface and the diffusion of the flexible polyether chain segments to 
the surface because they have a lower surface free energy than the hard segments. The 'melting' was thought to result from the lack of hard segment domains on the surface acting as temperature-stable filler. Whether or not the size and continuity of the brainlike texture is a result of the underlying hard segment distribution is not known.

SEM micrographs of untreated foam strut surfaces for foams 1 and 4 are shown in Figure 4.24. Surprisingly, the surfaces of the foams are very similar to those of the ion milled fracture surfaces. Evidently, the ion milling process increases the surface temperature to the extent that a very high degree of chain mobility is possible. As the surface recovers from ion milling it forms the surface texture of minimum energy or in this case, the texture that the surface of a foam would assume as it cures.

A more thorough investigation of the ion milling process is necessary before any definite conclusions can be made about the mechanisms involved. However, it does appear that in phase separated systems consisting of a continuous phase of very low $\mathrm{Tg}$, the ion milling process can increase the mobility of the soft segment to such an extent that a new surface is formed.

Lower magnification micrographs of a foam strut are shown in Figure 4.25. These micrographs reveal a higher level of texture (1 micron) oriented transverse to the axis of the strut. The roughness of the texture again provides evidence of a two phase morphology with some type of macrodispersed hard segment. 


\subsection{Swelling and Extraction}

The swelling of foams 5 and 6 by a number of different solvents is shown in Figure 4.26. In this figure, the increase in length of a rod of foam due to swelling by various solvents is presented and the solvents are listed by solubility parameter. Three solvents are significantly better than all the rest: THF; dimethyl acetamide; and dimethyl formamide. The percent elongation of foam 6 in THF is greater than the percent elongation of foam 5 in THF indicating that it is a better solvent for the polyol phase. Dimethyl acetamide and dimethyl foramide (DMF) are better solvents for the hard segment phase. THF is a cyclic ether and would be expected to be more compatible with the polyether polyols. The amide solvents are highly polar and have a carbonyl oxygen that can hydrogen bond with the polyurea hydrogens. These solvents are expected to be better hard phase solvents. When the aliphatic nature of the amide solvents is increased, their affinity for the foams decreases as evidenced in the data for $n$-dibutlyamide. The solubility parameter is not an effective method of predicting the affinity of a solvent for the foams as evidenced by the data for ethyl acetate and acetonitrile. Ethyl acetate has the same solubility parameter as THF but is a much poorer solvent. Acetonitrile is a poor solvent with a solubility parameter between that of two good solvents. An attempt was made to interpret 
the data in terms of separate contributions to the solubility due to dispersion forces, polar forces, and hydrogen bonding potential using values proposed by Hansen (46). Unfortunately, no correlation could be determined.

Extraction studies were carried out in THF and DMF and are summarized in Table 4.6. The sol fractions for THF extraction are much lower than those for DMF extraction indicating that much of the extract originates in the hard segments. This agrees with the conclusion from the $I R$ analysis of the extract that a large portion of the extract is urea and the observation that the sol fraction increases with increasing hard segment content. The low sol fractions observed in these experiments are evidence that the foams are comprised of a well-formed network.

Equilibrium swelling measurements of the plaques in DMF are summarized in Table 4.7. The plaques swelled isotropically and at equilibrium dimensions were enlarged by roughly fifty percent. The fact that the foams did not recover their previous cell structure is evidence that fusion took place during the compression molding process. The fully swollen weight increased slightly with hard segment content and time to reach equilibrium was inversely related to hard segment content. DMF has a higher affinity for hard segments and therefore it is surprising that the plaques with a higher hard segment absorbed DMF more slowly. Apparently the tighter packing and lower chain mobility in the hard segment outweigh the effect of 
more favorable interactions.

\subsection{WAXS}

Wide angle diffraction patterns for foams 1 through 4 are shown in Figure 4.27. All of the foams have a diffuse halo and a diffraction peak corresponding to 0.45 nanometers. Foams 2 through 4 have a second weaker peak at 0.59 nanometers. The peaks sharpen with increased hard segment content. A diffractometer scan of one of the compression molded plaques indicates that theses peaks are discrete peaks representing a certain degree of order and not just maxima of the amorphous halos (Fig. 4.28).

WAXS patterns for foams 5 and 6 are not shown but resembled foams 3 and 1 respectively. The WAXS patterns for the compression molded plaques resembled those of the respective foams. A brief annealing study was carried out in a attempt to sharpen the diffraction peaks. Foam 4 was annealed for 16 hours at $200 \mathrm{C}$. The WAXS pattern did not reveal significant changes.

WAXS patterns for plaque 4 before and after swelling are shown in Figure 4.29. The plaque that was exposed to the solvent does not have the 0.59 nanometer maximum and the peak at 0.45 nanometers is more diffuse than it was before swelling. The untreated plaque showed a diffuse halo centered about 1.6 nanometers. This had not 
been observed in any foams or plaques before. This is probably because they had shorter exposure times.

Several workers have used wide angle $x$-ray diffraction to study polyurethane-ureas with noncrystalline hard segments $(47,48,70)$. Both Wilkes (70) and Sung (47) showed that the hard segment was responsible for an amorphous halo with a maximum in the 0.4 to 0.5 nanometer range. A TDI-80 based water extended polyurea synthesized in this laboratory (28) had a broad amorphous halo in the 0.4 to 0.6 nanometer range and a narrow diffuse halo centered about 1.6 nanometers.

Based on the above studies, the amorphous halo in all the foams and plaques can be attributed to short range ordering in the hard segment. The 0.45 and 0.59 nanometer peaks are taken as an indicator of increased order of the hard segments. The origin of these peaks is unknown but is probably a result of noncrystalline ordering of the hard segments through hydrogen bonding. Similar peaks are observed in MDI-BD urethanes and are attributed to paracrystalline order (64), where paracrystalline ordering is not a minimum free energy state but results from the formation of a stable hydrogen bonding network.

The WAXS patterns did not change noticably as a result of compression molding or tin catalyst level indicating that these changes do not have a major effect on hard segment ordering. The swelling in DMF and subsequent drying of plaque 4 resulted in a decrease in hard 
segment order. One explanation for this is that the extraction process removed an unbound crystalline polyurea phase. A more likely explanation is that the presence of the solvent disrupted the hydrogen bonding network that was formed during foam rise and cure. The diffuse ring observed at 1.6 nanometers in untreated plaque 4 probably arises from the hard segment. A diffuse ring was observed for the polyurea at the same spacing. Spacings of this magnitude have been observed in MDI-BD urethanes and were attributed to the length of hard segment repeat unit.

\subsection{SAXS Analysis}

SAXS data for this foam project can be divided into four groups. The first three consist of data collected on the ORNL 10-meter SAXS instrument which features pinhole collimation and a two dimensional position-sensitive detector (43). The fourth group of data consists of complimentary measurements collected on a standard Kratky instrument $(28,33-35)$.

The first group of data was collected on a series of foams not discussed in this thesis. This data shows that foams alligned with the blow direction parallel to the incident beam scatter isotropically. Foams alligned with the blow direction perpendicular to the incident beam scatter anisotropically such that increased low angle scatter is observed along the blow direction. This could result form the 
presence of particles elongated in the blow direction or some other type of unaxial orientation along the blow axis. Based on these observations, care was taken to align the foams with the blow direction parallel to the incident beam in future SAXS experiments.

Data for the series $31135-33$ foams does not show this anisotropy. This could be the result of the deformation of the foam when it was compressed to increase the density of the foam in the $x$-ray beam. No anisotropy is observed for these foams in WAXS patterns when compressed samples are placed with the blow direction either parallel or perpendicular to the beam.

The second group of data consists of measurements on the ORNL 10-meter SAXS instrument for all six foams and plaques of series 31135-33. The incident intensity was determined by the method of foils and data was collected for a Lupolen standard. Lupolen is a specially annealed polyethylene material that is stable under extended exposure to $x$-rays and is used as a calibration standard $(74,75)$. The data was collected at a two meter sample-to-detector distance (SDD). On the ORNL 10-meter SAXS instrument, the two dimensional position-sensitive detector is of fixed size and is centered about the transmitted beam (zero scattering angle). As a result, the SDD determines the angular region over which data is collected. At long SDD's data is collected over a small angular region and at small SDD's data is collected over large angular regions. Since the detector is of fixed size, the resolution of the instrument is necessarily decreased as 
the angular region is increased.

The third group of data was collected at SDD's of 1 and 3 meters on the ORNL 10-meter SAXS instrument. Data was collected for foams and plaques 1 through 4 and for the foams of the blowing agent, polyol MW, and TDI-index studies. A Lupolen standard was run at both 1 and 3 meters for absolute intensity calibration. The 1 meter data for foams 1 through 4 was necessary to extend the angular region of the previously collected 2 meter data. Samples 5 and 6 were not included because analysis of the 2 meter data revealed that they are very similar to samples 3 and 1 respectively. Both 1 and 3 meter data were collected for the other foams in an effort to obtain the best possible combination of resolution and angular range.

For the fourth group of data a standard Kratky camera was used to obtain complimentary slit-smeared measurements for the foams and plaques of samples 1 and 4 .

\subsubsection{Treatment of Data}

The ORNL 10-meter SAXS instrument is interfaced with a computer and is very much a 'user oriented' instrument. Computer programs control data collection and treatment of the data and can be used to perform some basic SAXS analyses. In this investigation, the program CORRUN was used to correct the raw scattering data for parasitic scattering, electrical contributions to the scatter, and detector sensitivity. The output of the program is the radial average 
of the data collected on the two dimensional detector divided by the sample thickness $t$ and transmission $T$, and normalized to 1000 monitor counts (44).

$$
I_{\text {corrun }}=\frac{I_{\text {obs }} 1000}{\text { SMON Tt }}
$$

Further treatment of this data is discussed in terms of the 1 and 2 meter data collected for foams and plaques 1 through 4 . The 1 and 3 meter data taken for the supplemental foam series were treated in the same way. The slit-smeared data collected on the standard Kratky camera were analyzed using a computer program provided by Vonk (71). The analysis of the slit-smeared data is analogous to that of the pinhole data and is not discussed in detail below. For some of the analyses, the slit-smeared data had to be desmeared or the theory had to be modified to account for the slit-collimation.

\section{Absolute Intensity}

The observed intensity was scaled to an absolute intensity according to

$$
I(\kappa)=\frac{I_{o b s}(k) S D D^{2}}{I_{0} t T i_{e} a}
$$

where $l$ is the incident intensity, $i_{e}$ is the Thomson scattering factor, and $a$ is the area of the beam. When the scattering intensity is expressed in terms of the output from CORRUN, the above equation becomes 


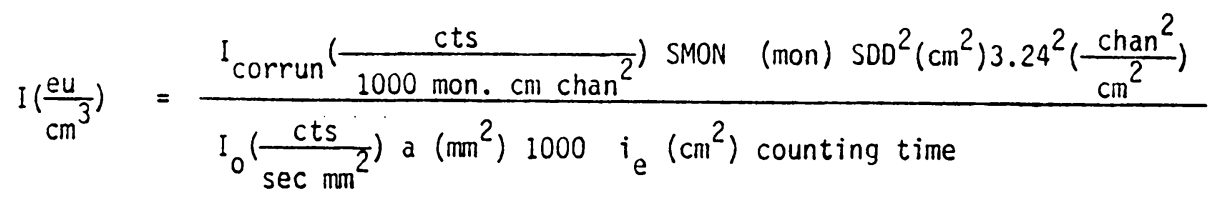

As stated earlier, the incident intensity was measured by the method of foils (72). In this experiment attenuating filters are placed in the beam path and the incident intensity is found by use of equation 2.2

$$
\begin{aligned}
& I(t) \equiv I_{0} \exp (-\mu t) \\
& \ln [I(t)]=\ln \left[I_{0}\right]-\mu t
\end{aligned}
$$

The incident intensity is the ordinate intercept in a plot of $\ln [1(t)]$ versus thickness.

The incident intensity could not be measured directly because it is too powerful for the detector. In the 2 meter geometry, the beam stop was removed from the face of the detector, foils were placed in the beam, and the intensity was measured for sixty seconds. The measured intensity was corrected for dark current IDC and the dead time $\tau$ of the detector electronics by

$$
I=\frac{I_{\text {mea }}-I_{D C}}{1-\tau\left(I_{\text {mea }}-I_{D C}\right)}
$$

where $\tau=7 \times 10^{-6}$ seconds (73). Measurements were made with from 5 to 12 nickel foils 1 micron thick. The incident intensity was determined to be $4.954 \times 10^{5} \mathrm{cts} /\left(\mathrm{sec} \mathrm{mm}^{2}\right)$.

\section{Combining Data Sets}


The preferred method of combining data sets is to adjust the intensity levels of each data set to absolute intensity. Absolute intensity adjustments can be based on either the actual measurement of the incident intensity or the use of a calibrated sample. The incident intensity was measured only for the 2 meter geometry and Lupolen standards were run for both geometries.

As mentioned before, Lupolen is a specially annealed polyethylene material that is stable under extended exposure to $x$-rays and is used as a calibration standard. The scattering profile for Lupolen exhibits a region where intensity drops off linearly with scattering angle. The center of this linear region corresponds to a d-spacing of 15 nanometers. A calibration constant based on this d-spacing is defined by

$$
K_{c}=I_{0} / I_{\text {obs }}(d=15 \text { nanometers })
$$

The absolute intensity is found by replacing 1 in equation 4.3 by the product $K_{c}{ }_{\text {obs }}(d=15)$.

From the 2 meter Lupolen data a value of $K_{c}$ was calculated and used to scale the 1 meter data to the level of the 2 meter data. This procedure worked fairly well for the plaques but did not work well for the foams. One explanation for this is that the measured intensity (from CORRUN) is normalized by dividing by the sample thickness. For the foam samples, the actual thickness of solid material that sees the $x$-ray beam is estimated from the thickness and density of the 
foam and the density of the corresponding plaque. In these experiments, foams approximately one centimeter thick were irradiated by a beam collimated to one square millimeter. The path of the beam was such that it traversed only about fifteen foam cells. This number of cells is not statistically great enough to insure that a representative proportion of windows, voids, and struts was seen by the beam. In other words, the actual amount of material in the $x$-ray beam is unknown.

To avoid this problem, the 1 meter data were scaled to the level of the 2 meter data by ratioing the data in the regions where the curves overlapped (specifically the Porod's law region from $k=1.0$ to 2.0 nanometers ${ }^{-1}$ ). This procedure was also applied to the plaques. The ratios or scale factors are shown in Table 4.8. All scale factors are based on at least twelve data points and have standard deviations of less than five percent. The low standard deviations and the closeness of the scale factors to the intensity ratio for the 1 and 2 meter Lupolen data at $d=15$ nanometers are taken as evidence of the validity of this method. The variance in the foam scale factors is taken as evidence that a problem exists in accounting for the amount of material in the beam.

Removal of Background Intensity

Background intensity, as discussed in chapter two, arises from the thermal motion of the scattering molecules. SAXS theory is based on scattering from fixed centers and therefore the background 
contribution must be removed before it can be applied. Methods of estimating the background intensity have been reviewed by Koberstein (29). Most of these methods rely on acquisition of data into the wide angle region. The 1 meter data from ORNL extends nearly to the onset of wide angle scattering but not far enough to use these techniques. The 2 meter data has a smaller angular region and does not extend close to the onset of wide angle scattering.

Ruland (76) and Bonart and Muller (30) have used an extension of Porod's law to estimate background intensity.

$$
\lim _{\kappa \rightarrow \infty} I_{\text {obs }}(\kappa) \kappa^{4}=K_{p} H^{2}(k)+I_{B} k^{4}
$$

In this method, data must extend beyond the angular region where the Porod's law damping function $H(k)$ becomes zero but not as far as the onset of wide angle scattering. The background intensity is a constant independent of scattering angle and is estimated from the slope of a plot of $I(k) k^{4}$ versus $k^{4}$. This method of estimation results in a slightly higher estimate of background intensity than the methods which require wide angle data. As a result, estimates of the diffuse boundary thickness will be slightly high.

The method of Ruland and Bonart was used to estimate the background intensity for the 1 meter data and the combined 1 and 2 meter data sets. The background intensity was removed from the observed intensity to yield the corrected intensity according to

$$
I(k)=I_{\text {correct }}(k)=I_{\text {obs }}(k)-I_{B}
$$


Figure 4.30 shows the angular regions encompassed by the 1 and 2 meter data. In Figure 4.31, this data is plotted to estimate the background intensity. From these plots it is evident that the extended angular region of the 1 meter data is required for a proper estimate of background intensity.

\section{Further Data Treatment}

The process of taking the inverse Fourier transform of scattering data requires integration over all $k$ where $k$ goes from zero to infinity. This requires estimation of a zero angle intensity and the acquisition of data to a high enough angle where the small angle intensity has dropped to zero. Extrapolation to zero scattering angle was accomplished through the Guinier analysis where the term $(\rho V)^{2}$ of equation 2.26 is equal to $1(0)$.

$$
I(\kappa)=(\rho v)^{2} \exp \left(-1 / 3 \quad \kappa^{2} R^{2}\right)
$$

Beyond the Porod's law region, the scattering profile was set equal to the values estimated by the Porod's law analysis.

$$
I(k)=\frac{k_{p}}{k^{4}} \exp \left(-\sigma^{2} \kappa^{4}\right)
$$

This assured a gradual damping of the scattered intensity with increasing scattering angle and is commonly done because of the poor counting statistics in the low scattering tail region. 
Finally, the process of taking the inverse Fourier transform of scattering data is also very sensitive to the point-to-point changes in slope due to the scatter of $x$-ray data. A cubic spline technique was used to smooth the data.

\subsubsection{Analysis of the Data}

Figures 4.32 and 4.33 show the scattering profiles for plaques 1 through 4 and foams 1 through 4 respectively. These curves are comprised of combined 1 and 2 meter data. They show correct relative intensity, and are the result of the previously described data treatment (background subtraction, zero angle extrapolation, tailfitting, smoothing). Several comments can be made about the foams and plaques just from the shapes of the scattering profiles. First, all of the scattering curves have a shoulder and the shoulder appears in approximately the same position for all the foams or plaques of the series. Each of these shoulders can be thought of as an interference peak that was not completely resolved due to occuring at too low of an angle and being too diffuse. The 2 to 100 nanometer morphological features that give rise to SAXS cannot be expected to be of the same size, shape, or separated by the same distances. As a result, sharp diffraction peaks are not seen in SAXS. By applying Bragg's law to the shoulders in Figures 4.32 and 4.33 an estimate of the center-tocenter distance between scattering particles is obtained. This distance for the plaques is approximately 10 nanometers and increases slightly with increasing water content. The d-spacing for the foams is 
roughly ten percent smaller at 9 nanometers and again increases with water content. These results are summarized in Table 4.9.

A second comment on the scattering profiles in Figures 4.32 and 4.33 is that the measured intensity increases with increasing water content. This is expected given that the contrast factor for small angle scattering from an ideal two phase system with sharp phase boundaries is given by

$$
\overline{\Delta \rho}^{2}=\phi(1) \Phi(2)[\rho(1)-\rho(2)]^{2}
$$

In the above expression, the contrast factor is called the mean-square electron density variance, $\rho(1)$ and $\rho(2)$ are the electron densities of phases 1 and 2, and $\phi(1)$ and $\phi(2)$ are volume fractions. For a given system $\rho(1)$ and $\rho(2)$ are constant and the volume fractions determine the amount of contrast. Since $\phi(1)$ and $\phi(2)$ must sum to one, the contrast reaches a maximum for a fifty-fifty mixture of phases and decreases as the volume fractions become less even. Also note that the contrast is independent of which phase has the higher electron density. In Figure 4.32, the intensity increases as hard segment content of the plaques increases. In Figure 4.33, the expected behavior is observed except foam 3 intensity is greater than foam 4 .

One explanation for the transposition of foams 3 and 4 is that the measured intensity is improperly normalized by dividing by the thickness (corrected with bulk density) of the foam. This was mentioned above as the probable cause of problems in scaling the 1 
and 2 meter data. The $x$-ray beam does not traverse a statistically great enough number of cells to insure that a representative proportion of windows, voids, and struts is always seen by the beam. As a result, there is a degree of uncertainty in knowing how much material is exposed to the $x$-ray beam.

A final observation about the general shape of the scattering curves regards the difference in low angle scattering between foams and plaques. The plaques show a zero angle intensity that is roughly two to three times the shoulder intensity. The foams show a zero angle intensity that is roughly eight to fifteen times the shoulder intensity. This increased low angle intensity is believed to arise from both surface reflections and Bragg's law interferences due to the presence of voids (cell structure) and not the morphology within the struts of the foam. It adversely affects the SAXS analyses associated with curve-fitting this portion of the scattering profile or integrating the scattering profile. The extra low angle scattering should not affect the position of interference peaks or any analyses concerned with the tail region of the scattering profile.

\section{Correlation Function}

The correlation functions give the probability that a rod of length $R$ will have both ends in regions of the same electron density. They are calculated by integrating the scattering profile for contributions due to scattering centers a distance $R$ apart and then dividing by the integrated intensity for all interactions as discussed in chapter two 
(eqns. 2.30,2.32). Because the correlation functions are ratios of integrals, the influence of the increased low angle intensity in the foam data may be normalized out. If the increased low angle scatter does influence the correlation function it would shift the order to larger $d$-spacings (lower angle, larger spacing). The first maximum in a 3-dimensional correlation function should correspond to the interparticle spacing given by Bragg's law. The first maximum in a 1-dimensional correlation function gives the spacing between scattering centers with a lamellar morphology.

Both correlation functions were calculated for the foams and plaques. The results are listed along with the Bragg's law spacings in Table 4.9. The positions of the first maxima in the 3-dimensional correlational functions are less than the Bragg's law spacing for both the foams and plaques. This is generally observed $(28,78)$ and may arise from the the relative weighting of different sized or shaped particles by the two methods. The first maximum of the 3-dimensional correlation function is thought to be the better estimate of center-tocenter particle spacing.

The correlation functions for plaque 3 are shown in Figure 4.34. The 3-D correlation function shows a weak maximum at 9.5 nanometers. The breadth of this peak suggests a distribution of interdomain spacings. The 1-D correlation function has a much sharper maximum located at 7.3 nanometers. Neither correlation function shows repeated maxima indicating a lack of long range order. 
The correlation distances for the foams are smaller than for the plaques indicating that the increased low angle scatter did not appreciably affect the calculations. The $3-D$ and 1-D correlation functions for foam 3 are shown in Figure 4.35. The 3-D correlation function has a maximum at 7.7 nanometers. This lower maximum may be the result of the correlation function not dropping below zero before the maximum. Comparison of the 3-D correlation functions for the foam and plaques suggests a more continuous distribution of hard segments in the foams. The 1-D correlation function has a maximum at 6.9 nanometers. As with the plaques, there is little periodicity in the correlation functions suggesting a lack of long range order.

The correlation functions for the other foams and plaques resemble Figures 4.34 and 4.35 closely. The correlation distances generally increase slightly with hard segment content suggesting the presence of slightly larger domains. The smaller correlation distances of the foams as compared to the plaques could be the result of the orientation of the foam in the beam. The anisotropic scattering data discussed earlier suggests that the domains may be elongated in the blow direction and thus would appear smaller when observed looking down the blow axis. In the plaques these domains would be randomly oriented and on the average appear larger. The larger correlation distances in the plaques could also result from improved phase separation and the formation of larger domains during the compression molding process. 
In TDI-80 based urethane or urethane-urea elastomers $d$-spacings twenty to fifty percent higher were observed $(36,78)$. These systems were extended by ethylene glycol or ethylene diamine and were made with linear polyols. The shorter, stiffer hard segments in the foams and the use of trifunctional polyols both would act to hinder the formation of large domains as compared to the elastomers. The smaller $d$-spacings in the foams are, on this basis, reasonable.

\section{Guinier Analysis}

The size of the scattering particles was estimated using the Guinier analysis. In this treatment, the radius of gyration of the scattering particles is proportional to the slope of a plot of $\ln [1]$ versus $k^{2}$. The steeper the negative slope, the larger the scattering particles. A Guinier plot for plaque 2 is shown in Figure 4.36.

The Guinier analysis, as discussed in chapter two, is closely obeyed over a large angular region for a dilute system of monodisperse spheres. In polydisperse systems and systems with anisotropic scattering particles, a Guinier plot will become nonlinear with increasing scattering angle. The limiting slope, however, as $k$ approaches zero is still related to the mean square radius of gyration. The approximations made in deriving Guinier's law are valid when the limiting slope is determined in a region such that the product $k * R g$ is less than $1.3(77)$.

The results of the Guinier analysis are summarized in Table 4.9. 
The plaques all have radii of gyration of about 4.5 nanometers. If the particles were monodisperse spheres they would have a diameter of roughly 12 nanometers. At first glance, this does not agree with the interdomain spacings of roughly 9 nanometers. However, polydispersity and anisotropy lead to high measurements of radii of gyration and anisotropic particles such as rods have much larger radii of gyration per unit volume than spheres.

Physically, a 0.8 inch diameter sphere in a 1.0 inch cube occupies roughly twentyfive volume percent. A system consisting of twentyfive volume percent of 0.8 inch diameter equally dispersed spheres would have a Bragg spacing of only 1.0 inch. Therefore, the radii of gyration and Bragg spacings of the plaques are not out of line considering the polydispersity and anisotropy expected in the hard phase.

The radii of gyration for the foams again do not vary with hard segment content and are all roughly 8.4 nanometers. These values were obtained just outside the range of validity for the Guinier approximation. This is not important because the magnitude of these radii of gyration are thought to reflect the increase in low angle scatter observed in the foams rather that the morphology of the solid portions of the foam.

Diffuse Boundary Thickness

According to Porod's law, the scattered intensity for a two phase 
system with sharp phase boundaries in the limit of high scattering angle (tail region) will obey:

$$
\lim _{k \rightarrow \infty} I_{p}(k)=\frac{k_{p}}{k^{4}}
$$

where $k$ is the scattering angle and $K p$ is a constant related to the area of interface per unit volume. In the limit of high scattering angle, the product on the left hand side of the above equation should be constant. When this product increases with increasing scattering angle, the material is said to show positive deviations from Porod's law. Positive deviations can result from thermal fluctuations (background intensity) and from the isolation of a unit of one phase in the wrong phase (phase mixing). Negative deviations result from a diffuse boundary between phases.

To characterize the diffuse boundary, the scattering contribution from background intensity must be subtracted and the scattering from phase mixing must be assumed negligible. This assumption may not be valid as suggested by analysis of a series of foams of constant water content and increasing TDI-index (Fig. 4.37). As the TDI-index increased larger positive deviations from Porod's law are observed indicating increased phase mixing. At constant index levels, as in the 31135-33-X series, the degree of phase mixing is not known but can be assumed constant and relative values of the diffuse boundary thickness can be calculated.

Diffuse boundary thicknesses were calculated assuming a sigmoidal 
gradient for the domain interface. The observed scattering data was fit to the product of a smoothing function and ideal Porod's law scatter. Sigma is the interfacial parameter that comes out of this treatment. Sigma is the half width of the diffuse boundary where the smoothing function has a value of 0.606 (Fig. 2.7). The length of the diffuse boundary assuming a linear gradient is roughly 3.5 times sigma.

The results of the Porod's law analysis are summarized in Table 4.10. The values calculated from the combined 1-meter and 2-meter data are more accurate. For the foam series, foam 1 shows a sigma value of 0.23 nanometers and the sigma values increase with hard segment content up to 0.53 nanometers for foam 4 . The reverse trend might be expected based on the formation of larger, better-ordered domains with increasing hard segment content. The observed trend indicates that the lengths of the chain-extended hard segments might become more polydisperse with increasing water content and that improved ordering may not be important with such an assymetric hard segment. The plaques have slightly larger diffuse boundaries and show the same trend with increasing hard segment content. The slightly larger boundary thicknesses in the plaques point again toward larger domains in the plaques. These sigma values are of the same size as values determined for TDI-based urethane and urethane-urea elastomers $(36,78,79)$.

Degree of Phase Separation 
The mean square electron density variance is the contrast factor for SAXS. For a completely phase separated two phase system with sharp boundaries the electron density variance is given by

$$
\overline{\Delta p}^{2}=\phi(1) \phi(2)[o(1)-\rho(2)]^{2}
$$

Theoretically, this electron density variance can be calculated from the pure hard and soft segment electron densities and the volume fractions of these phases.

In real systems, the contrast factor can be diminished by the presence of diffuse phase boundaries and incomplete phase separation (phase mixing). Experimentally, the contrast factor is determined by

$$
\overline{\Delta \rho}^{2}=\frac{1}{2 \pi^{2} V} \int_{0}^{\infty} I(\kappa) \kappa^{2} d \kappa
$$

In the Porod's law analysis, the contribution of the diffuse phase boundaries to the small angle scattering is determined. If this contribution is taken into account an electron density variance that reflects only phase separation can be determined by

$$
\overline{\Delta p} 2^{\prime \prime}=\frac{1}{2 \pi^{2} V} \quad \int_{0}^{\infty} \frac{I(k) k^{2}}{H^{2}(k)} d k
$$

As discussed in chapter two, ratios of these electron density variances can give a qualitative estimate as to the degree and state of phase separation .

$\overline{\Delta \rho^{2}} / \overline{\Delta \rho^{2}} \quad$ degree of phase separation

${\overline{\Delta \rho^{2}}}^{\prime \prime} /{\overline{\Delta \rho^{2}}}^{\prime}-1$ pressence of diffuse boundary 
$\overline{\Delta \rho^{2}} /{\overline{\Delta \rho^{2}}}^{\prime \prime}-1$ presence of phase mixing

In theory, the above ratios should be zero for no phase separation, sharp boundaries, or no phase mixing and increase with increasing phase separation, boundary diffuseness, or phase mixing. A value of one for the ratio in equation 2.40 would be the maximum representing a state of complete phase separation.

The theoretical electron density variance for a completely phase separated system is calculated from volume fractions and the electron densities of the pure phase. Volume fractions are estimated from the chemical composition of the material and assumed pure phase densities. Electron densities are calculated from pure phase densities, the MW's of the repeat units, and the number of electrons in the repeat units (28). The problem with these calculations is estimation of the pure phase densities. Since the electron density variance is proportional to the square of the difference in electron densities, proper estimation of pure phase densities is very important.

The soft phase density was assumed to be equal to the $3000 \mathrm{MW}$ polyol density of $1.016 \mathrm{~g} / \mathrm{cc}$. This results in an electron density of 0.561 mole electrons/cc.

The hard phase density was much more difficult to approximate. Based on the known weight percent hard segment, the measured density of the plaques, and the 'assumed' soft segment density the 
density of the hard segment was determined to be $1.22 \mathrm{~g} / \mathrm{cc}$. Use of this hard segment density results in very low electron density variances as compared to Koberstein (36) and Bonart (80). The plaques show traces of the cell structure that existed before compression molding. These voids lower the plaque density and therefore lower the calculated hard segment density. Bonart calculated hard segment densities as high as $1.44 \mathrm{~g} / \mathrm{cc}$ for an MDIhydrazine system. In lieu of a better estimate for hard segment density a value of $1.50 \mathrm{~g} / \mathrm{cc}$ was assumed. The calculated electron density is 0.791 mole electrons/cc and the theoretical variances are on the order of 0.0060 (mole electrons $/ \mathrm{cc})^{2}$. These are close to those reported by the above authors. Because of the assumptions used to calculate the theoretical variances, ratios involving this term can only be interpreted on a relative basis.

In Table 4.12 the theoretical electron density variances, the experimentally determined electron density variances, and the ratios describing phase separation are summarized. For the plaques, the degree of phase separation is independent of hard segment content which agrees with the DMS soft segment $\mathrm{Tg}$ measurements. The degree of phase separation ratios are only relative, but the low soft segment $\mathrm{Tg}$ values from DMS suggest a high level of phase separation. The phase mixing ratios suggest that phase mixing decreases with hard segment content. This may be related to the higher softening temperatures observed in DMS with higher hard segment content. Finally, interface mixing increases with hard segment content. This is 
somewhat surprising because phase separation improves with increasing hard segment content, but could result from the greater dispersity in hard segment lengths expected at higher hard segment contents.

The experimentally determined electron density variances for the foams are 35 to 100 percent greater than those of the corresponding plaques. This probably arises from the increased low angle scatter in the foams. One explanation for the increased low angle scatter is the macroscopic cell structure of the foams. But, if the foam was scattering as a two phase system of solid and voids, much higher electron densities would be expected. Another possibility is the formation of smaller voids in the solid portions of the foam. These voids could occur during curing as unreacted isocyanate reacts with moisture to produce carbon dioxide. Foams with more TDI would be expected to have more voids. This agrees with the observation that the difference between foam and plaque variances increases with hard segment content. The presence of voids has not, however, been noted in SEM and TEM investigations.

The increased low angle scatter may also be the result of surface reflections from the strut and burst membrane surfaces alligned parallel to the beam. The foams with higher hard segment content have a finer cell structure. This would result in more surfaces for reflection agreeing with the increasing difference between foam and plaque variances with increasing hard segment. 
Data was collected for foams and plaques 1 and 4 on a standard Kratky camera and analyzed using a program provided by Vonk (71). The Kratky data, in contrast to the pinhole data, show much less defined shoulders and more of a linear decrease in intensity with scattering angle (Fig. 4.38). Most of this is a consequence of the slit collimation which smears the features of small angle scattering profiles as compared to pinhole collimation. The Kratky camera data does show a small peak in the 3 to 4 nanometer region which could be a lower order reflection originating from higher $d$-spacings. This peak did not exist in the pinhole data and verification of its existence was not possible due to an inability to obtain more ORNL beam time. This peak falls in the Porod's law region which makes background estimation difficult and makes the determination of a diffuse boundary thickness impossible. The detailed analysis of this data is not presented here in view of the above problems.

Several comparisons of the Kratky and pinhole data are still possible. The Bragg's law d-spacings and correlation distances are slightly smaller in the Kratky data but are still of the same order of magnitude. The electron density variances of the plaques are in good agreement with the pinhole data, but those for the foams are much higher than the pinhole data. This is probably because the slitcollimation exposes $x$-rays to many more surfaces than a pinhole beam even though the areas of the beams are about equal.

The effect of annealing on morphology was studied with a Kratky 
camera equipped with a position-sensitive detector. Small angle scattering measurements were made for plaque 4 before annealing, immediately after annealing for 8 minutes at $200 \mathrm{C}$ and quenching, and 24 hours after annealing. Similar measurements were taken for a sample annealed for 12 hours at $92 \mathrm{C}$ and then quenched. No major changes were observed in the small angle scattering profiles indicating that the thermal treatments did not significantly alter the degree of phase separation or ordering of domains in these systems.

\subsection{Summary}

A series of six foams was analyzed by a number of techniques in an effort to learn more about the morphology of these systems. In foams 1 through 4 the water content was systematically increased from $2 \mathrm{pph}$ in foam 1 to $5 \mathrm{pph}$ in foam 4 . The catalyst and surfactant levels were held constant and the TDI-index was fixed at 110 . In foams 5 and 6 , the tin catalyst levels of foams 3 and 1 were adjusted to yield optimum foams. The adjustments in tin catalyst levels did not change the foams very much and as a result the focus of this work is on the first four foams.

Plaques were made of all the foams by compression molding at 204 C for 10 minutes. The advantage of the plaques is that they allow analysis of the material independent of cell geometry. There is, however, some uncertainty as to how the morphology of the foam 
changes on compression molding.

The SEM investigation revealed that as the water level is increased, the cells become slightly smaller and are more elongated. The cell struts become much thinner and the foams become more open.

The thinning of the cell struts and the increasing openess of the foam are reflected in the density and air flow measurements respectively. Other mechanical property measurements reveal behavior analogous to that of a typical urethane elastomer. As the water level (hard segment content) increases the strength and tear resistance improve and the hysteresis behavior declines. The modulus, defined as the ratio of $65 \%$ ILD to $25 \%$ ILD, decreases only slightly with hard segment content. Because of the large changes in strut thicknesses with increasing water content a large decrease in modulus would be expected. This can be taken as indirect evidence that the material comprising the struts increases in strength with increasing water content.

Dynamic mechanical spectroscopy showed that a high level of phase separation is obtained, that phase separation is independent of hard segment content, and that the hard segment is stable to over $200 \mathrm{C}$. The stability of the hard segment increases slightly with hard segment content suggesting the presence of larger or better ordered domains. A loss transition was observed around $80 \mathrm{C}$ for some of the samples. It only appeared on the first scan and is attributed to the loss of adsorbed moisture. 
DMS spectra for the plaques reveal slightly lower soft segment Tg's and slightly higher hard segment softening temperatures as compared to the foams. This suggests that phase separation and hard segment ordering is improved slightly by the compression molding process.

Differential scanning calorimetry confirmed the independence of soft segment $\mathrm{Tg}$ from hard segment content and the increase in hard segment softening temperatures with increasing hard segment content. A small endotherm was observed at $80 \mathrm{C}$ on the first scan of all of the foams. The endotherm did not appear on the second scan or if the sample was annealed at $120 \mathrm{C}$ before scanning. This endotherm is thought to be the source of the $80 \mathrm{C}$ loss transition and further work is needed to confirm that it is caused by adsorbed moisture.

The FTIR study revealed that there is unreacted isocyanate in the foams a month after they are manufactured. The unreacted isocyanate groups are probably in the stiff, glassy hard segment domains through which diffusion of a reacting species is slow. The unreacted isocyanate concentration decreases with compression molding and with humid-aging. The compression molding process also changes the carbonyl region. This could be a result of changes in the hydrogen bonding structure or the loss of thermally liable crosslinks such as allophanates or biurets.

Thin films approximately 120 nanometers thick were cut from the foams and plaques and observed using TEM. The micrograph of foam 
1 exhibited a grainy texture suggesting that there may be order of a much smaller dimension than the thickness of the specimen. This agrees with the observation that plaque 1 is transparent to light. The TEM micrograph for foam 2 shows hard segment dense regions roughly 100 nanometers in size that are randomly dispersed. There are agglomerations of these 100 nanometer regions and regions of intermediate contrast that tend to suggest a semicontinuous nature to the hard segment. The micrographs for foams 3 and 4 are very similar to the micrographs for foam 2, but show better contrast, the presence of more hard segment, and the presence of larger agglomerations. These TEM micrographs agree with the observation that the opacity of the plaques increases with hard segment. The TEM micrographs for the plaques are very similar to those of the corresponding foams. The sizes of the features observed in TEM correspond well with textures observed on cold fracture surfaces with a scanning electron microscope.

Swelling and extraction experiments showed that the foams are indeed networks. The sol fractions are small and increase with hard segment concentration. The sol fractions are greater than the weight of excess isocyanate and unbound catalysts and surfactants indicating that all molecules capable of being incorporated into the network are not. Analysis of the extract reveals that is is mostly irea.

WAXS patterns for foams 1 through 4 reveal diffuse rings that are thought to represent some type of order in the hard segment. All 
foams show a diffraction maximum at 0.45 nanometers. Foams 2 through 4 show a second, less intense maximum at 0.59 nanometers. The clarity of the peaks increases with hard segment content. These peaks could not be noticably sharpened by annealing. The WAXS pattern for a sample of plaque 4 that was swollen with DMF and then dried lost the 0.59 nanometer maximum and showed a more diffuse 0.45 nanometer maximum. This is attributed to the solvent breaking up a hydrogen bonding network that was formed during swelling.

The SAXS analysis revealed that the foams have interparticle distances of about 7 nanometers. These spacings increase slightly with hard segment content suggesting the presence of slightly larger domains. The foams have diffuse boundary thicknesses that increase with hard segment content and range from 0.23 to 0.53 nanometers. The increase in diffuse boundary thickness with increasing hard segment content is thought to arise from an increasing dispersity in hard segment lengths.

The plaques have significantly larger interparticle distances ( 9 nanometers) and only slightly larger diffuse boundary thicknesses. The difference in interparticle distances can be attributed to either increased domain sizes as a result of compression molding or the loss of uniaxial orientation along the blow direction on compression molding. A more detailed study of the anisotropy of these foams is needed. The degree of phase separation in the plaques is independent of hard segment content agreeing with the DMS data. This may be due to a 
combination of increased phase mixing at lower hard segment contents and more diffused boundaries at higher hard segment contents.

\subsection{Conclusions and Recommendations}

The solid material that comprises these foams is a phase separated system with exceptional elastomeric properties under small deformations. The exceptional elastomeric properties are probably more a result of the extreme incompatibility of the chain extended urea and polyol phases than a result of the unique foaming kinetics. The work of Sung $(47,48)$ and Tyagi $(28)$ with urethane and urethaneurea elastomers is evidence of this. A combination of their work shows the vast improvements in phase separation from systems with urethane extended hard segments to urea extended hard segments to finally urea extended hard segments with no aliphatic character such as in the foams.

The degree of phase separation in these foams is very high and is independent of hard segment content. This conclusion is supported by DMS, DSC, and SAXS data as well as the work of Tyagi (28) on urethane-urea elastomers.

From the SAXS analysis, the center-to-center spacing between hard segment domains is roughly 7 nanometers and increases slightly with hard segment content. The boundaries between phases vary from 
roughly one-third of a hard segment repeat unit in thickness for foam 1 to greater than two-thirds of a hard segment repeat unit for foam 4 . The correlation distances are smaller than in typical urethane elastomers but are expected to be smaller based on the short chain extender and the anisotropy of TDI. The boundary thicknesses are similar to those observed in typical urethane elastomers.

The plaque corresponding to foam 1 is transparent suggesting the absence of structures on the order of the wavelength of light. The TEM micrographs for foam 1 suggest that order exists that is much smaller that the 120 nanometer thickness of the specimen. This data along with the SAXS and DMS studies suggests that foam 1 is a two phase system with isolated hard segment domains of less than 7 nanometers.

In plaques 2, 3, and 4, opacity increases with increasing hard segment content indicating the presence of a macrostructure on the order of the wavelength of light. TEM micrographs also show the presence of large groupings which increase in size and concentration with increasing hard segment content. The TEM micrographs are supported by SEM micrographs of cold fracture surfaces. These groupings are between one and two orders of magnitude larger than the dimensions expressed as the 'expected' morphology.

The large particles are probably urea precipitates as suggested by Kollmeier and coworkers (15). The particles acting as filler account for the systematic changes in the mechanical properties. The particles 
are embedded in an elastomeric matrix that is described by the SAXS results. The similar correlation distances all the foams suggests that hard segment domain sizes do not vary much with hard segment content. Therefore, the increasing hard segment content is reflected in the size and amount of precipitate. If the urea precipitates are significantly large and spherical in shape they would have a constant surface to volume ratio in each foam. As a result, the trend expressed in the diffuse boundary layer analysis would still be correct. The larger diffuse boundaries in the foams with higher water content may represent more polydisperse chain extension.

The compression molding process does not significantly alter the morphology of the foams, but the additional thermal history does result in minor changes. The degree of phase separation and high temperature stability does improve on compression molding and the FTIR study shows changes in the carbonyl region. The correlation distances in the plaques are larger than in the foams. This might be due to the loss of some orientation. Hard segment order, as expressed by WAXS patterns, does not change with compression molding.

Future work should begin with the assumption that there is a binodal distribution of hard segment domains in the foams and plaques. There are large urea precipitates that act as filler and smaller, more conventional domains that act as virtual crosslinks. 
The presence of such a large amount of urea in the precipitated particles suggests that upon formation of a dimer, the urea precipitates. If this is true the average hard segment unit would be only two or three aromatic units long. An analogous foam could be made by using isocyanate-capped polyols and a polyurea filler. Work along these lines was done by Kollmeier et al $(15,18)$. More importantly, if the source of compression set is hydrogen bonding to the polyurea filler other types of filler could be used in its place. Along these same lines, when trifunctional polyols are used what is the role of the virtual crosslinks?

The formation of urea precipitates could be studied by measuring the solubility of polyurea in the foam-mix as a function of degree of chain extension.

With the same series of foams there are still alot of unanswered questions. The loss transition observed in DMS and the endotherm observed in DSC cannot definitely be attributed to adsorbed moisture. A TGA study of these foams could be used to detect a weight loss in this temperature region. Similarly, annealed foams could be aged in the atmosphere and under dessicant and then scanned on the DSC for the presence of this endotherm.

The surfaces of the plaques and foams before and after extraction with DMF could be observed under SEM to provide additional evidence for the existence of the large urea precipitates. 
A detailed study of the anisotropy in the foams should be carried out. The 31135-33 series of foams did not show appreciable anisotropy on the ORNL pinhole SAXS instrument, but this may be the result of compressing the foam before placing it in the beam. Also, the origin of the increased low and scatter in the foams should be investigated.

The hard segment order as described by WAXS should be studied further. The fact that the ordering increases with hard segment content suggests that the ordering is in the urea precipitate phase. The presence of the 1.6 nanometer ring needs to be confirmed. It could be indicative of hard segment sizes. The 1.6 nanometer spacing would suggest a hard segment chain extension of just two units. Also, WAXS patterns could be taken at higher temperatures to investigate the temperature stability of the observed hard segment ordering. 


\section{REFERENCES}

1. G.B. Woods,"Flexible Polyurethane Foams: Chemistry and Technology," Applied Science Publishers, LTD, New Jersey (1982).

2. K.C. Frisch, J.H. Saunders,"Plastic Foams: Part 1," Marcel Decker, Inc., New York (1972).

3. K.C. Frisch, J.H. Saunders,"Plastic Foams: Part 2," Marcel Decker, Inc., New York (1972).

4. B.A. Dombrow, "Polyurethanes," 2nd ed., Reinhold, New York (1965).

5. A.A. Berlin, F.A. Shutov, A.K. Zhitinkina, "Foam Based on Reactive Oligomers," Technomic Pub. Co.,Inc., Connecticut (1982).

6. Dow Chemical Co., "The Flexible Foam Handbook," Dow Chemical U.S.A., Urethanes Dept., Midland, MI.

7. H.J. Twitchett, Chem. Soc. Review, $\underline{3}(\underline{2}), 209$ (1974).

8. G. Burkhart, H.J. Kollmeier, H.H. Schleons, J. Cellular Plastics, 20(1), 37 (1984).

9. C.E. McGinn, R.G. Spaunburgh, Symposium on Isocyanate Polymers, ACS Meeting, Atlantic City, N.J. (1956).

10. R. Baumhakel, J. Cellular Plastics, $\underline{8}(\underline{6}), 304$ (1972).

11. G. Hauptmann, K.H. Dorner, H. Hocker, G. Pfisterer, "Cellular and Non-sellular Polyurethanes," International Conference, Strassbourg, France, Urethane Division, SPI, 635 (1980).

12. F.E. Bailey, Jr., F.E. Critchfield, J. Cellular Plastics, 17( $\underline{6})$, 333 (1981).

13. V.V. Zarkov, L.I. Kopusov, E.A. Petrov, "Cellular and Nonsellular Polyurethanes," International Conference, Strassbourg, France, Urethane Division, SPI, 635 (1980).

14. G.R. Rossmy, H.J. Kollmeier, W. Lidy, H. Schator, M. Wiemann, J. Cellular Plastics, 13(1), 26 (1977).

15. G.R. Rossmy, H.J. Kollmeier, W. Lidy, H. Schator, M. Wiemann, J. Cellular Plastics, 17(ㅌ) , 319 (1981).

16. P. Van Gheluwe, J. LeRoux, J. Appl. Poly. Sci., 28, 2053 (1983). 
17. R.L. Rowton, J. Cellular Plastics, 16, 287 (1980).

18. H.J. Kollmeier, G. Burkhart, J. Kietsch, H. Lammerting, J. Cellular Plastics, 20(6) $) 410$ (1984).

19. G. Rossmy, W. Lidy, H. Schator, M. Wiemann, H.J. Kollmeier, J. Cellular Plastics, 15(ㅁ) , 276 (1979).

20. H.J. Kollmeier, H. Schator, P. Zaeske, SPI 27th Annual Technical/Marketing Conference Proceedings, Bal Harbor, Florida, 251 (1982).

21. B. Kanner, B. Prokai, C.S. Eschbach, G.J. Murphy, J. Cellular Plastics, 15(ㅇ) , 315 (1979).

22. A. Guinier, Ann. Phys., 12, 161 (1939).

23. P. Bebye, A.M. Bueche, Appl. Phys., 20, 518 (1949).

24. G. Porod, Kolloid Z., 124, 83 (1951).

25. G. Porod, Kolloid Z., 125, 51 (1952).

26. G. Porod, Kolloid Z., 125, 108 (1952).

27. W. Ruland, J. Appl. Crystallogr., 4, 70 (1971).

28. D. Tyagi, Ph D Thesis, Virginia Polytechnic Institute and State University, Chemical Engineering Dept. (1985).

29. J.T. Koberstein, B. Morra, R.S. Stein, J. Appl. Crystallogr., 13, 34 (1980).

30. R. Bonart, E.H. Mueller, J. Macromol. Sci. Phys., B10, 177 (1974).

31. R. Bonart, E.H. Mueller, J. Macromol. Sci. Phys., B10, 345 (1974).

32. B.D. Cullity, "Elements of X-ray Diffraction," Addison-Wesley Publishing Co., Inc., Reading, Mass. (1978).

33. L.E. Alexander, "X-ray Diffraction Methods in Polymer Science," R.E. Kreiger Publishing Co., Huntington, New York (1979).

34. M. Kakuda, N. Kasai, "X-ray Diffraction by Polymers," Elsevier Publishing Co., New York (1972).

35. O. Glatter, O Kratky, ed., "Small Angle X-ray Scattering," Academic Press, New York (1982).

36. J.T Koberstein, R.S. Stein, J. Polymer Sci. Phys., 21, 1439 (1983). 
37. J.T Koberstein, R.S. Stein, J. Polymer Sci. Phys., 21, 2181 (1983).

38. J.S. Higgins, R.S. Stein, J. Appl. Crystallog., 11, 346 (1978).

39. G.L. Wilkes, S Abouzahr, D. Radovich, J. Cellular Plastics, $\underline{19}(\underline{4}), 248$ (1983).

40. E. Helfand, Acc. Chem. Res., 8, 295 (1975).

41. E. Helfand, Y. Tagami, Polymer Lett., 9, 741 (1971).

42. S.L. Aggarwal, R.A. Livigni, L.F. Marker, T.J. Dudek, "Block and Graft Copolymers," J.J. Burke, V. Weiss, eds., Syracuse University Press, New York, 157 (1973).

43. R.W. Hendricks, J. Appl. Crystallogr., 11, 15 (1978).

44. NCSASR, "User Notes for the 10-Meter SAXS Instrument," ORNL, Oak Ridge, Tenn. (1983).

45. O. Kratky in "Small Angle X-ray Scattering," M. Glatter, $O$. Kratky, eds., Academic Press, New York (1982).

46. J. Brandrup, E.H. Immergut, eds.,"Polymer Handbook," 2nd ed., John Wiley and Sons, New York (1975).

47. C.S. Paik Sung, C.B. Hu, C.S. Wu, Macromolecules, 13, 111 (1980).

48. N.S. Schneider, C.S. Paik Sung, R.W. Matton, J.L. Illinger, Macromolecules, 8(1), 62 (1975).

49. P.J. Flory, "Principles of Polymer Chemistry," Cornell University Press, Ithaca, New York (1953).

50. G.M. Estes, S.L. Cooper, A.V. Tobolsky, J. Macromol. Sci., C4 (2) $) 313(1970)$.

51. D.S. Huh, S.L. Cooper, Polymer Eng. Sci., 11(ㅁ), 369 (1971).

52. C.G. Seefried, J.V. Koleske, F.E. Critchfield, J. App/. Polymer Sci., 19, 2493 (1975).

53. C.G. Seefried, J.V. Koleske, F.E. Critchfield, J. App/. Polymer Sci., 19, 2503 (1975).

54. C.G. Seefried, J.V. Koleske, F.E. Critchfield, J. App/. Polymer Sci., 19, 3185 (1975).

55. R.W. Seymour, S.L. Cooper, Macromolecules, 6 , 48 (1973). 
56. H.N. Ng, A.E. Allegrezza, R.W. Seymour, S.L. Cooper, Polymer, 14, 255 (1973).

57. T.R. Hesketh, J.W.C. Van Bogart, S.L. Cooper, Polymer Eng. Sci., 20( $\underline{3}), 190$ (1980).

58. N.S. Schneider, C.S. Paik Sung, Polymer Eng. Sci., 17(2), 73 (1977).

59. C.S. Paik Sung, T.W. Smith, N.H. Sung, Macromolecules, 13, 117 (1980).

60. S.L. Cooper, D.S. Huli, W.J. Morris, Ind. Eng. Chem. Prod. Res. Dev., I, 248 (1968).

61. K. Knutson, D.J. Lyman in "Biomaterials: Interfacial Phenomena and Applications," S.L. Cooper, ed., Advances in Chemistry Series 199, ACS, Washington D.C. (1982).

62. C.B. Wang, S.L. Cooper, Macromolecules, 16, 775 (1983).

63. C.S. Paik Sung, N.S. Schneider, Macromolecules, 8, 68 (1975).

64. R. Bonart, L. Morbitzer, E.H. Muller, J. Macromol. Sci. Phys., B9 (3), 447 (1974).

65. N.S. Schneider, C.R. Desper, J.L. Illinger, A.O. King, J. Macromol. Sci. Phys., B11(4), 527 (1975).

66. A.L. Chang, E.L. Thomas in "Multiphase Polymers," S.L. Cooper, ed. Advances in Chemistry Series 176, ACS, Washington, D.C. (1979).

67. I.D. Fridman, E.L. Thomas, Polymer, 21(4), 389 (1980).

68. A.L. Chang, R.M. Briber, E.L. Thomas, R.J. Zdrahala, F.E. Critchfield, Polymer, 23, 1060 (1982).

69. L.A. Koutsky, N.V. Hein, S.L. Cooper, J. Polymer Sci. Lett., B8 $(\underline{5}), 353$ (1970).

70. Y.P. Chang, G.L. Wilkes, J. Polymer Sci.Phys., 13, 455 (1975).

71. C.G. Vonk, J. Appl. Crystallogr., 8, 340 (1975).

72. V. Luzzati, Acta. Cryst., 13, 939 (1960).

73. M.Y. Tang, PhD Thesis, U. of Tennessee (1983).

74. I. Pilz, O. Kratky, J. Colloid and Interface Sci., 24, 211 (1967).

75. I. Pilz, J. Colloid and Interface Sci., 30, 140 (1969). 
76. W. Ruland, J. Appl. Crystallogr., 4, 70 (1971).

77. A. Guinier, G. Fournet, "Small Angle Scattering of $X$-Rays," John Wiley and Sons, Inc., London (1955).

78. G.L. Wilkes, S. Abouzahr, Macromol., 14, 458 (1981).

79. J.T. Koberstiein, R.S. Stein, Polymer Eng. Sci., 24(ㅁ), 293 (1984).

80. R. Bonart, E.H. Muller, J. Macromol. Sci. Phys., B10(2), 345 (1974).

81. R.B. Turner, H.L. Spell, G.L. Wilkes, SPI 28th Annual Technical/Marketing Conference 244 (1984). 
Table 3.1: Description of Formulation Components

Polyol: Voranol 3100, a $3000 \mathrm{MW}$ propylene oxide glycerine initiated polyether polyol

from Dow Chemical (approx. trifunctional)

Isocyanate: $\quad T-80$, an $80: 20$ mixture of the 2,4- and 2,6-isomers of toluene diisocyanate from Dow Chemical

Water/

Blowing Agent

Deionized water, no blowing agent used

Catalysts:

$T-9$, a tin catalyst from $M \& T$ Chemical (stannous octoate)

DABCO 33LV, an amine catalyst from Air Products (33\% DABCO or triethylenediamine in dipropylene glycol)

Surfactant: $\quad$ BF-2370, a silicone surfactant from Goldschmidt 
Table 3.2: Formulations for Series 31135-33-X Foams and Plaques

$\begin{array}{lcccccc}\text { Foam Number X } & 1 & 2 & 3 & 4 & 5 & 6 \\ \begin{array}{l}\text { Voranol 3100 } \\ \text { Water }\end{array} & 100 & 100 & 100 & 100 & 100 & 100 \\ & 2.0 & 3.0 & 4.0 & 5.0 & 4.0 & 2.0 \\ \begin{array}{l}\text { Surfactant } \\ \text { BF-2370 }\end{array} & 1.0 & 1.0 & 1.0 & 1.0 & 1.0 & 1.0 \\ \begin{array}{l}\text { Amine Catalyst } \\ \text { DABCO 33LV }\end{array} & 0.3 & 0.3 & 0.3 & 0.3 & 0.3 & 0.3 \\ \begin{array}{l}\text { Tin Catalyst } \\ \text { T-9 }\end{array} & 0.15 & 0.15 & 0.15 & 0.15 & 0.175 & 0.10 \\ \begin{array}{l}\text { Isocyanate } \\ \text { T-80 }\end{array} & 30.79 & 41.43 & 52.06 & 62.70 & 52.06 & 30.79\end{array}$

\section{Comments :}

1) Formulations base on 100 parts by weight of polyol.

2) In all foams the isocyanate index is 110.

3) In foams 1 through 4 only water and isocyanate content vary at constant isocyanate index.

4) In foams 5 and 6 the foams contain the optimal tin concentration.

5) Plaques are made by compression molding foams at 250 tons of pressure and $204 \mathrm{C}$ for 10 minutes. 
Table 3.3 Formulations for the Blowing Agent, Polyol MW, and TDI Index Studies

Blowing Agent Study: Series 31857-37-X
Foam $X$
pph Water
pph Freon
tin catalyst
1
2
5.0
0.0
0.175
3.0
15.0
0.200

Polyol MW Study: Series 31857-40-X

$\begin{array}{ccl}\text { Foam } X & \text { pph Water } & \text { Polyol } \\ 1 & 4.0 & 2000 \mathrm{MW} \text { trifunctional PPO } \\ 2 & 4.0 & 3000 \mathrm{MW} \text { trifunctional PPO }\end{array}$

TDI Index Study: Series 31857-45-X

$\begin{array}{cccc}\text { Foam } X & \text { pph Water } & \text { TDI Index } & \text { tin catalyst* } \\ 5 & 4.0 & 95 & -- \\ 2 & 4.0 & 105 & -- \\ 3 & 4.0 & 115 & -- \\ 6 & 4.0 & 125 & --\end{array}$

* tin catalyst level was slightly decreased as TDI index was increased 
Table 4.1: Average Chain Dimensions for Series 31135-33 Foams Complete reaction of all water and polyol with TDI is assumed. Excess isocyanate is neglected.

$\begin{array}{ccccc}\text { Foam } & \begin{array}{c}\text { \# units } \\ \text { 2 PPO arms }\end{array} & \begin{array}{c}\text { length } \\ \text { (nanometers) }\end{array} & \begin{array}{c}\text { \# rings } \\ \text { hard segment }\end{array} & \begin{array}{c}\text { length } \\ \text { (nanometers) }\end{array} \\ 1 & 34.4 & 12.0 & 3.2 & 2.2 \\ 2 & 34.4 & 12.0 & 4.3 & 3.0 \\ 3 & 34.4 & 12.0 & 5.4 & 3.8 \\ 4 & 34.4 & 12.0 & 6.5 & 4.5 \\ 5 & 34.4 & 12.0 & 5.4 & 3.8 \\ 6 & 34.4 & 12.0 & 3.2 & 2.2\end{array}$

Table 4.2: Composition of Series 31135-33 Foams Weight and volume percent hard segment were calculated neglecting excess isocyanate.

Foam pph water wto hard seg. vol\% hard seg.

$\begin{array}{llll}1 & 2 & 21.1 & 17.5 \\ 2 & 3 & 25.8 & 21.4 \\ 3 & 4 & 30.1 & 25.0 \\ 4 & 5 & 33.8 & 28.0 \\ 5 & 4 & 30.1 & 25.0 \\ 6 & 2 & 21.1 & 17.5\end{array}$

Comments: 1. Hard segment is considered as everthing between urethane ether oxygens.

2. The excess TDI would increase values by about ten percent.

3. Volume fractions are based on densities of $1.016 \mathrm{~g} / \mathrm{cc}$ for SS and $1.22 \mathrm{~g} / \mathrm{cc}$ for HS. 
Table 4.3 Mechanical Properties of Series 31135-33 Foams

\begin{tabular}{|c|c|c|c|c|c|c|}
\hline Foam & 1 & 2 & 3 & 4 & 5 & 6 \\
\hline pph water & 2 & 3 & 4 & 5 & 4 & 2 \\
\hline pph tin catalyst & 0.15 & 0.15 & 0.15 & 0.15 & 0.175 & 0.10 \\
\hline density (lbs/ft3) & - & 1.92 & 1.43 & 1.24 & 1.47 & 2.85 \\
\hline tensile (psi) & - & 12.7 & 13.9 & 13.9 & 13.7 & 10.4 \\
\hline \% elongation & - & 138 & 159 & 157 & 156 & 112 \\
\hline tear (lb/in) & - & 1.5 & 1.8 & 1.8 & 1.9 & 1.1 \\
\hline resilience $(\stackrel{\circ}{\circ})$ & - & 53 & 49 & 47 & 49 & 58 \\
\hline $90 \% \mathrm{CT}$ (set) & - & 1.8 & 2.6 & 3.4 & 2.1 & 1.5 \\
\hline $25 \%$ ILD & - & 47 & 41 & 25 & 35 & 42 \\
\hline $65^{\circ}$ ILD & - & 81 & 69 & 65 & 57 & 69 \\
\hline $25^{\circ} \mathrm{RTN}$ ILD & - & 33 & 27 & 22 & 27 & 41 \\
\hline hysteresis & - & 70 & 66 & 63 & 64 & 77 \\
\hline modulus & - & 1.72 & 1.68 & 1.63 & 1.64 & 1.89 \\
\hline guide factor & - & 24.5 & 28.7 & 28.3 & 28.5 & 18.6 \\
\hline air flow & - & 3.2 & 4.6 & 5.9 & 4.5 & 1.9 \\
\hline
\end{tabular}


Table 4.4a Mechanical Properties of Series 31857-37 and 40 Blowing Agent (37) and Polyol MW Studies (40)

$\begin{array}{lrrrr}\text { Foam } & 37-1 & 37-2 & 40-1 & 40-2 \\ \text { pph water } & 5.0 & 3.0 & 4.0 & 4.0 \\ \text { pph Freon } & 0.0 & 15.0 & 0.0 & 0.0 \\ \text { polyol MW } & 3000 & 3000 & 2000 & 3000 \\ \text { density (Ibs/ft3) } & 1.29 & 1.28 & 1.52 & 1.46 \\ \text { tensile (psi) } & 11.4 & 7.9 & 9.9 & 12.8 \\ \text { \% elongation } & 111 & 108 & 86 & 75 \\ \text { tear (Ibs/in) } & 1.4 & 0.9 & 1.0 & 1.0 \\ \text { resilience (\%) } & 44 & 54 & 45 & 21 \\ \text { 90\% CT (set) } & -- & -- & -- & -- \\ \text { 25\% ILD } & 39 & 21 & 54 & 68 \\ \text { 65\% ILD } & 62 & 38 & 90 & 120 \\ \text { 25\% RTN ILD } & 24 & 16 & 34 & 43 \\ \text { hysteresis } & 62 & 76 & 63 & 62 \\ \text { modulus } & 1.59 & 1.81 & 1.67 & 1.76 \\ \text { guide factor } & 30.2 & 16.4 & 35.5 & 46.6 \\ \text { air flow } & 2.5 & 3.1 & 2.4 & 2.5\end{array}$


Table 4.4b Mechanical Properties of Series 31857-45 TDI-Index Study

\begin{tabular}{lrrrr} 
Foam & $45-5$ & $45-2$ & $45-3$ & $45-6$ \\
Pph water & 4.0 & 4.0 & 4.0 & 4.0 \\
TDI-index & 95 & 105 & 115 & 125 \\
density (Ibs/ft3) & 1.79 & 1.79 & 2.03 & 1.94 \\
tensile (psi) & 10.9 & 9.4 & 15.3 & 12.4 \\
o elongation & 86 & 59 & 38 & 36 \\
tear (Ibs/in) & 1.0 & 0.9 & 1.3 & 0.6 \\
resilience (\%) & 48 & 45 & 44 & 39 \\
90\% CT (set) & 3.4 & 3.5 & 12.1 & 8.6 \\
25\% ILD & 55 & 57 & 80 & 110 \\
65\% ILD & 93 & 98 & 173 & 264 \\
25\% RTN ILD & 37 & 38 & 57 & 44 \\
hysteresis & 67 & 67 & 71 & 40 \\
modulus & 1.69 & 1.72 & 2.16 & 2.40 \\
guide factor & 30.8 & 31.9 & 39.4 & 56.8 \\
air flow & 3.6 & 3.7 & 2.7 & 0.1 \\
\hline
\end{tabular}


Table 4.5 Thermal Transitions of Series 31135-33 Samples

\begin{tabular}{lrrrr} 
Foam & \multicolumn{2}{c}{$\begin{array}{c}\text { Tg } \\
\text { by DMS }\end{array}$} & $\begin{array}{c}\text { Tg } \\
\text { by DSC }\end{array}$ & $\begin{array}{c}\text { Thard) } \\
\text { by DMS }\end{array}$ \\
1 & & & & \\
2 & -40 & $(-43)$ & -44.3 & 235 \\
3 & -38 & $(-40)$ & -46.4 & 245 \\
4 & -38 & $(-42)$ & -47.3 & 243 \\
4 & -41 & $(-42)$ & -47.9 & 250
\end{tabular}

Comments: 1. Values in parentheses are for plaques.

2. $\mathrm{Tg}$ is based on peak in $\tan \delta$.

3. T(hard) is hard segment softening temperature.

4. All temperatures are degrees $C$. 
Table 4.6: Sol Fractions in DMF and THF

$\begin{array}{lcc}\text { sample } & \text { in DMF }\left(\begin{array}{l}\% \\ \%\end{array}\right) & \text { in THF }(\%) \\ \text { foam } 1 & 2.2 & 0.5 \\ \text { foam } 4 & 6.7 & 0.5 \\ \text { plaque } 1 & 2.2 & -\cdots \\ \text { plaque } 4 & 4.2 & -\cdots\end{array}$

Table 4.7: Equilibrium Swelling Data

$\begin{array}{cccc}\text { plaque } & w t(e q) / w t(0) & I(e q) / I(0) & \begin{array}{c}\text { time to equil } \\ \text { (hrs) }\end{array} \\ 1 & 3.54 & 1.49 & 5 \\ 2 & 3.60 & 1.51 & 6 \\ 3 & 3.73 & 1.51 & 8 \\ 4 & 3.85 & 1.54 & 8\end{array}$


Table 4.8: Scale factors used to scale 1 meter data to the level of the 2 meter data.

$\begin{array}{ccc}\text { sample } & \begin{array}{c}I(2 \mathrm{~m}) / I(1 \mathrm{~m}) \\ \text { foam }\end{array} & \begin{array}{c}I(2 \mathrm{~m}) / I(1 \mathrm{~m}) \\ \text { plaque }\end{array} \\ 1 & 0.52 & 0.41 \\ 2 & 0.38 & 0.39 \\ 3 & 0.66 & 0.36 \\ 4 & 0.40 & 0.34 \\ \text { Lupolen } & 0.46 \text { at } \mathrm{d}=15 \text { nanometers }\end{array}$

Table 4.9: Interparticle Spacing

d Bragg's law

$R$ 3-D Correlation Function, first maximum

$X$ 1-D Correlation Function, first maximum

$\begin{array}{crlll}\text { Foam } & d & R & X \text { nanometers } \\ 1 & 8.9 & 6.9 & 6.6 \\ 2 & 9.4 & 7.3 & 6.7 \\ 3 & 9.4 & 7.7 & 6.9 \\ 4 & 9.6 & 7.2 & 7.1 & \\ & & & & \\ \text { Plaque } & \mathrm{d} & \mathrm{R} & \times \quad \text { nanometers } \\ 1 & 10.0 & 9.0 & 6.9 \\ 2 & 9.9 & 9.1 & 7.0 \\ 3 & 10.2 & 9.5 & 7.3 \\ 4 & 10.6 & 9.6 & 7.4\end{array}$


Table 4.10: Particle Size by Guinier Analysis

Guinier approximation is only valid when when $k * R g$ is less than 1.3.

$\begin{array}{lcc}\text { Foam } & \begin{array}{c}\mathrm{Rg} \\ (\mathrm{nm})\end{array} & k^{*} \mathrm{Rg} \\ 1 & 8.4 & 1.35 \\ 2 & 8.4 & 1.35 \\ 3 & 8.3 & 1.33 \\ 4 & 8.3 & 1.33 \\ \text { Plaque } & & k * \mathrm{Rg} \\ & \mathrm{Rg} & \\ 1 & (\mathrm{~nm}) & \\ 2 & 4.7 & 0.89 \\ 3 & 4.3 & 0.82 \\ 4 & 4.2 & 0.80 \\ & 4.5 & 0.86\end{array}$

Table 4.11: Diffuse Boundary Thickness

By analysis of deviations from Porod's law assuming a sigmoidal gradient. (for a linear gradient $E=\sigma \sqrt{ } 12$ )

\begin{tabular}{|c|c|c|c|}
\hline Foam & $\begin{array}{l}\text { Sigma } \\
(1 \mathrm{~m})\end{array}$ & $\begin{array}{l}\text { Sigma } \\
(1 \& 2 m)\end{array}$ & nanometers \\
\hline 1 & 0.23 & 0.23 & \\
\hline 2 & 0.40 & 0.39 & \\
\hline 3 & 0.51 & 0.44 & \\
\hline 4 & 0.52 & 0.53 & \\
\hline Plaque & $\begin{array}{c}\text { Sigma } \\
(1 \mathrm{~m})\end{array}$ & $\begin{array}{l}\text { Sigma } \\
(1 \& 2 m)\end{array}$ & nanometers \\
\hline 1 & 0.30 & 0.27 & \\
\hline 2 & 0.43 & 0.42 & \\
\hline 3 & 0.53 & 0.49 & \\
\hline 4 & 0.57 & 0.53 & \\
\hline
\end{tabular}


Table 4.12: Evaluation of Degree of Phase Separation

Electron Density Variances (mole electrons $/ \mathrm{cc})^{2}$

$\begin{array}{cccc}\text { Foam } & \Delta \rho^{2} & \Delta \rho^{21} & \Delta \rho^{2 \prime} \\ 1 & 0.0050 & 0.0045 & 0.0050 \\ 2 & 0.0056 & 0.0061 & 0.0080 \\ 3 & 0.0063 & 0.0090 & 0.0122 \\ 4 & 0.0068 & 0.0070 & 0.0104 \\ & & & \\ \text { Plaque } & \Delta \rho^{2} & \Delta \rho^{2} & \Delta \rho^{2 "} \\ & & & \\ 1 & 0.0050 & 0.0033 & 0.0038 \\ 2 & 0.0056 & 0.0038 & 0.0051 \\ 3 & 0.0063 & 0.0042 & 0.0060 \\ 4 & 0.0068 & 0.0047 & 0.0070\end{array}$

Degree of Phase Separation

(based on above electron density variances)

\begin{tabular}{|c|c|c|c|}
\hline Foam & $\begin{array}{l}\text { phase } \\
\text { sep. }\end{array}$ & $\begin{array}{l}\text { phase } \\
\text { mixing }\end{array}$ & $\begin{array}{l}\text { interface } \\
\text { mixing }\end{array}$ \\
\hline 1 & -- & -- & 0.11 \\
\hline 2 & -- & - & 0.30 \\
\hline 3 & -- & -- & 0.35 \\
\hline 4 & -- & -- & 0.50 \\
\hline Plaque & $\begin{array}{l}\text { phase } \\
\text { sep. }\end{array}$ & $\begin{array}{l}\text { phase } \\
\text { mixing }\end{array}$ & $\begin{array}{l}\text { interface } \\
\text { mixing }\end{array}$ \\
\hline 1 & 0.67 & 0.30 & 0.15 \\
\hline 2 & 0.68 & 0.10 & 0.35 \\
\hline 3 & 0.66 & 0.06 & 0.44 \\
\hline 4 & 0.68 & -0.02 & 0.50 \\
\hline
\end{tabular}


a) $\mathrm{R}-\mathrm{NCO}+\mathrm{R}^{\prime}-\mathrm{OH} \longrightarrow \stackrel{\mathrm{H} O}{\mathrm{R}-\dot{N}-\mathrm{C}^{\prime \prime}-\mathrm{O}-\mathrm{R}^{\prime}}$

urethane

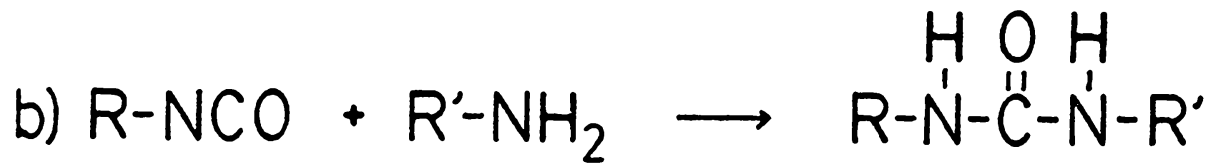

disubstituted urea

c) $\mathrm{R}-\mathrm{NCO}+\mathrm{H}_{2} \mathrm{O}$
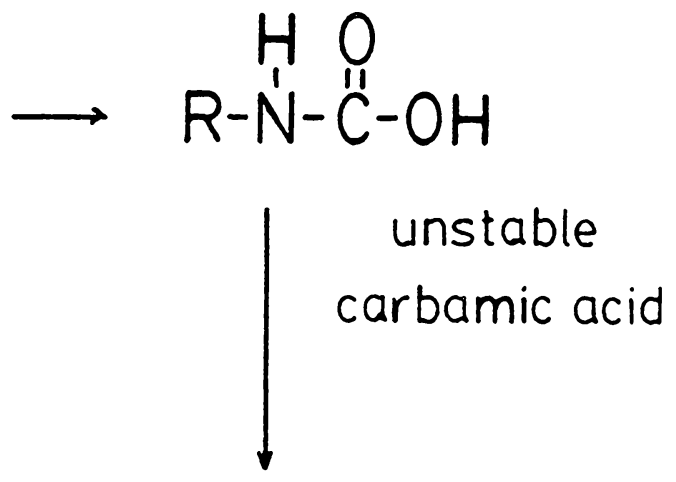

$\mathrm{R}-\mathrm{NH}_{2}+\mathrm{CO}_{2}^{\uparrow}$

Figure 1.1: Primary reactions in flexible foam manufacture. 


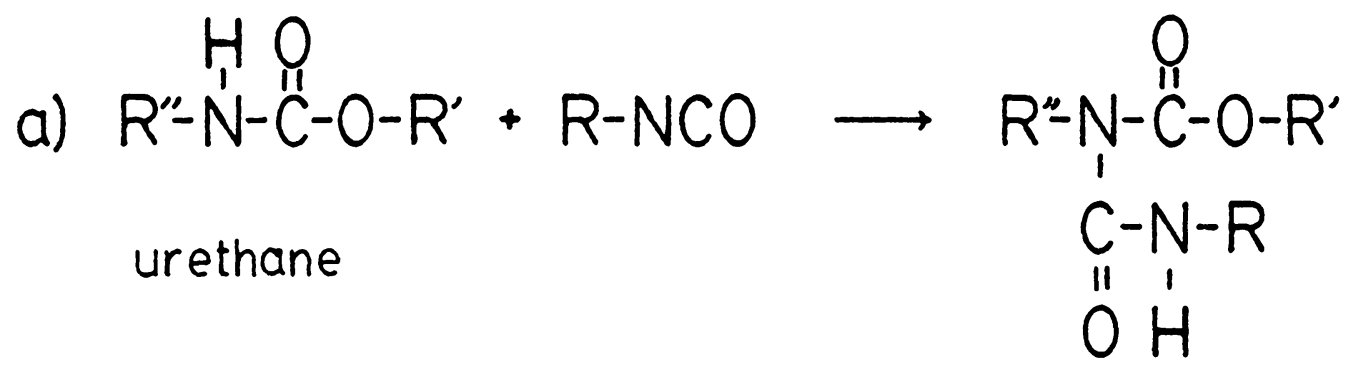

allophanate

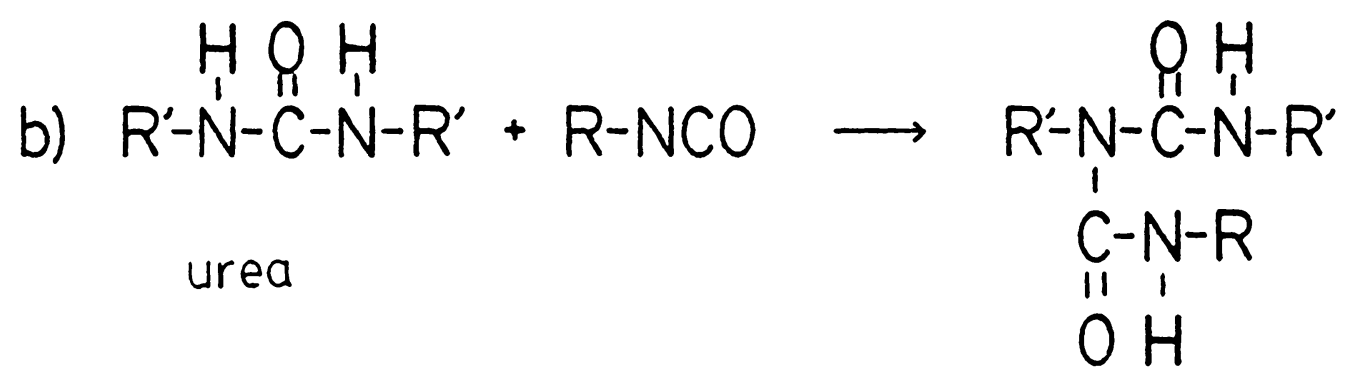

biuret

Figure 1.2: Important secondary reactions in flexible foam manufacture. 
polyethyleneoxide (PEO)

$$
\mathrm{fCH}_{2}-\mathrm{CH}_{2}-\mathrm{Of}_{X}
$$

polypropyleneoxide (PPO)
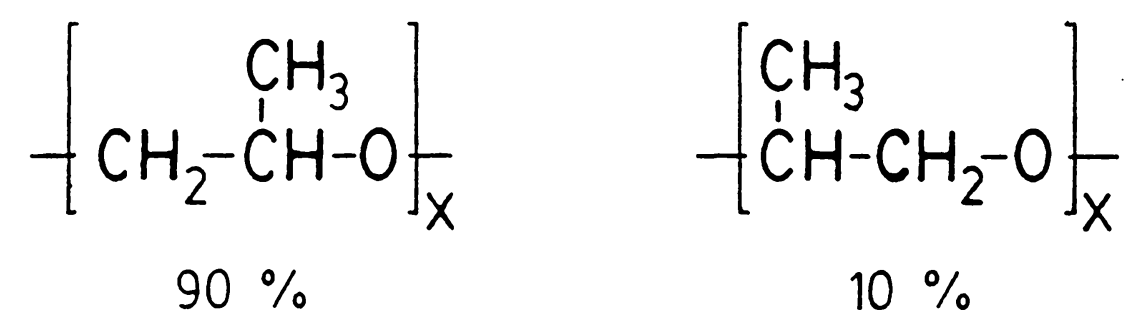

Figure 1.3: Repeat units for most common polyether polyols.

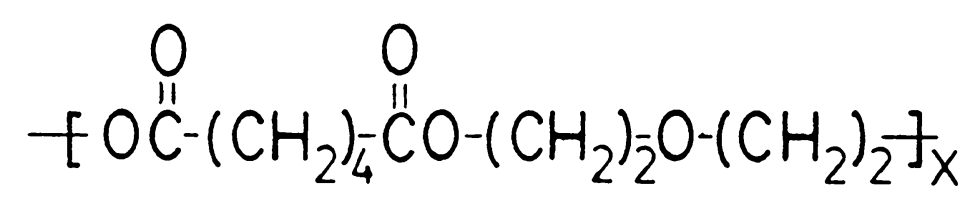

poly(oxydiethylene adipate)

Figure 1.4: Repeat unit for most common polyester polyol. 
toluene diisocyanate<smiles>Cc1ccc(N=O)cc1NC=O</smiles>

2,4<smiles>Cc1c(N=O)cccc1N=O</smiles>

2,6

4,4-diphenylmethane diisocyanate (MDI)<smiles>[C]c1ccc(N=O)cc1</smiles>

polymeric $\mathrm{MDI}$<smiles>O=CNc1ccc(Cc2ccc(N=O)cc2)cc1</smiles><smiles>O=Cc1ccc(Cc2ccccc2N=O)cc1</smiles><smiles>[Y]C([Y])(c1ccc([N+](=O)[O-])cc1)c1cc(CC)ccc1[N+](=O)[O-]</smiles>

Figure 1.5: Structures of isocyanates commonly used in flexible foam manufacture. 

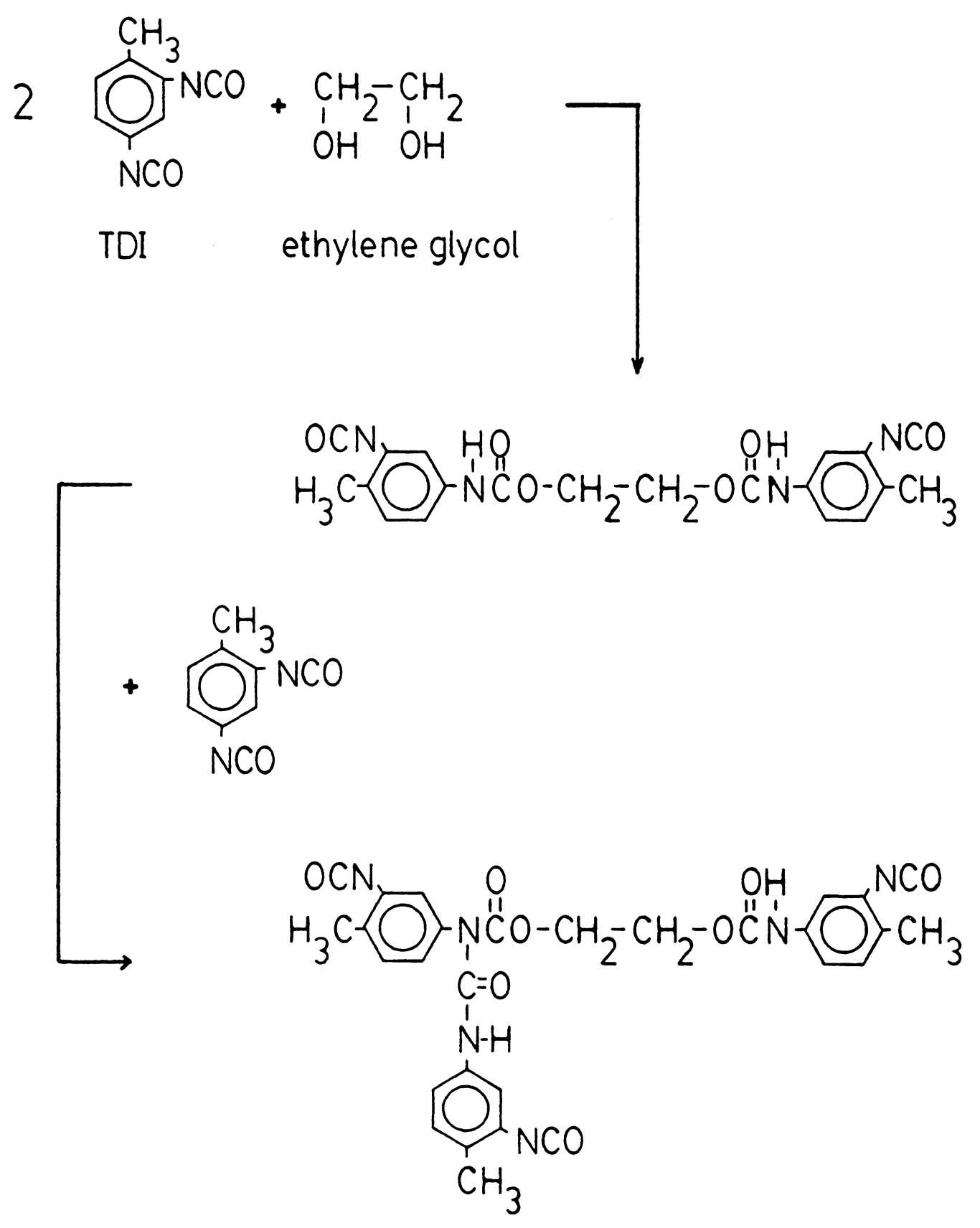

Figure 1.6: Synthesis of allophanate modified TDI. 
$3 \mathrm{R}-\mathrm{NCO} \longrightarrow$

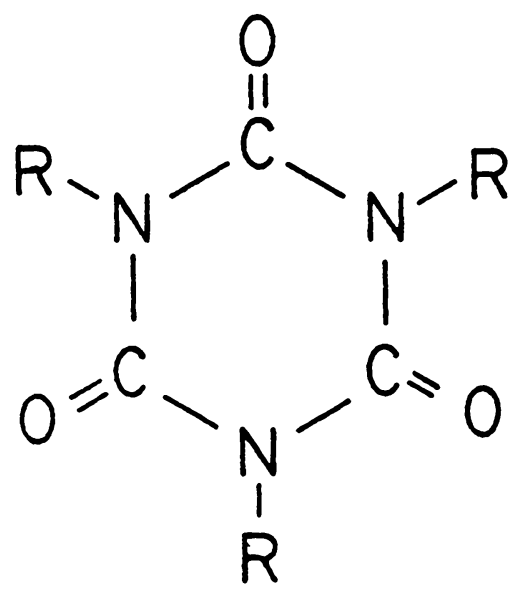

Figure 1.7: Formation of isocyanurates. 
<smiles>C1CN2CCN(C1)C2</smiles>

$\left(\underset{\mathrm{CH}_{3}}{\mathrm{CH}_{3}} \stackrel{\mathrm{CH}_{2} \mathrm{CH}_{2}}{\mathrm{CH}_{3}}\right)_{2} \mathrm{NCH}_{3}$<smiles>CN(C)CCN(C)CCO</smiles>

$\left(\underset{\mathrm{N}_{3}}{\mathrm{NH}_{3}} \mathrm{CH}_{2} \mathrm{CH}_{2}\right)_{2} \mathrm{O}$<smiles>CN(C)CCO</smiles>

Diazabicyclo(2.2.2)octan, or Trlethylenedamine. TEDA

Pentamethyldipropylenetriamine

Trimeinyl-hydroxyeinyl-einylenediamine

BIs /dimethylaminoeinyllether

Dimethylethanolamine. DMEA

Figure 1.8: Tertiary amine used in flexible foam manufacture. They are ordered from top to bottom in terms of increasing selectivity towards the blowing reaction (ref. 8). 


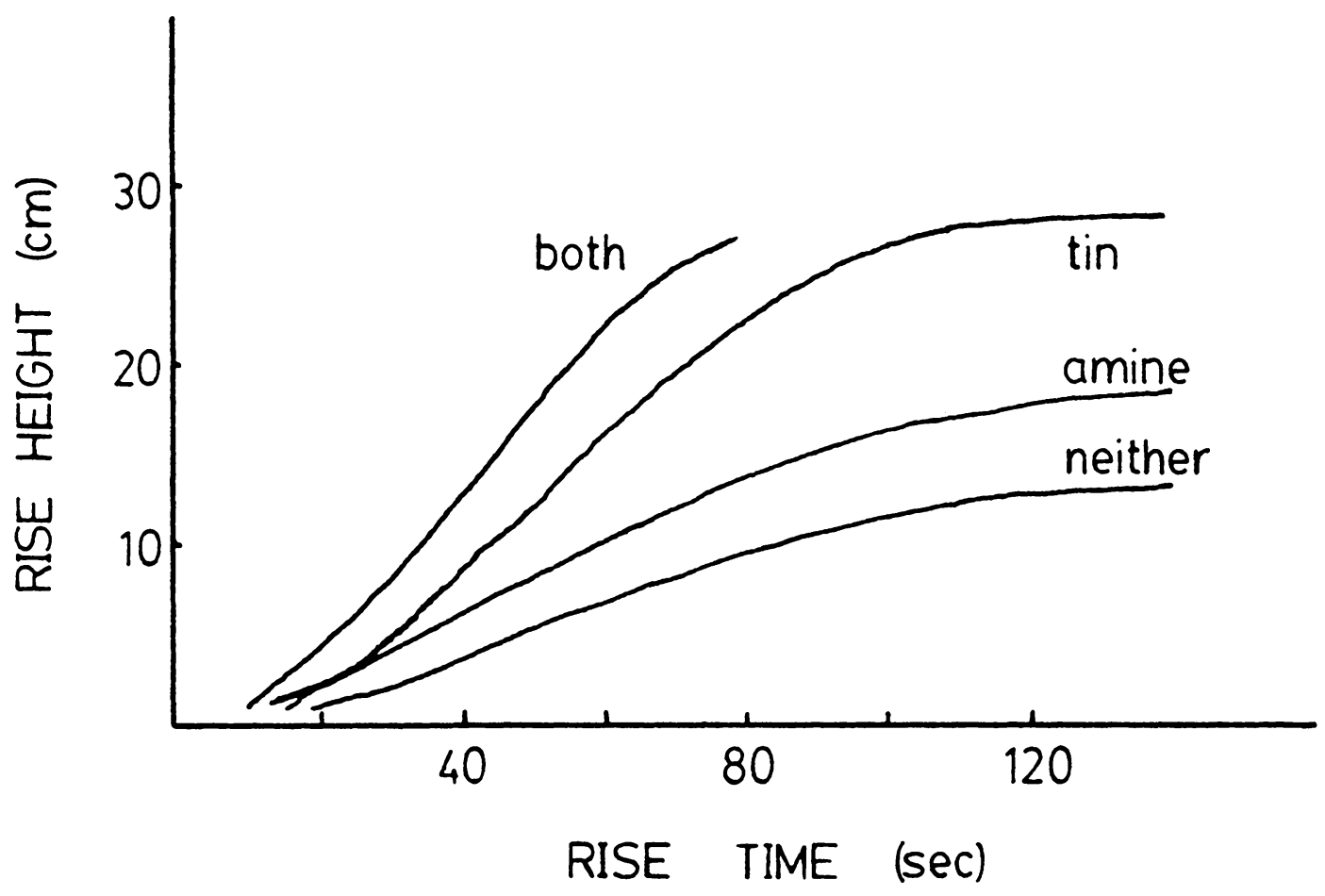

Figure 1.9: Importance of catalyst system in manufacture of a 5 pph water foam, $0.2 \mathrm{pph}$ tin catalyst (stannous octoate), and 0.2 pph amine catalyst (dimethylethanolamine) (ref. 8). 

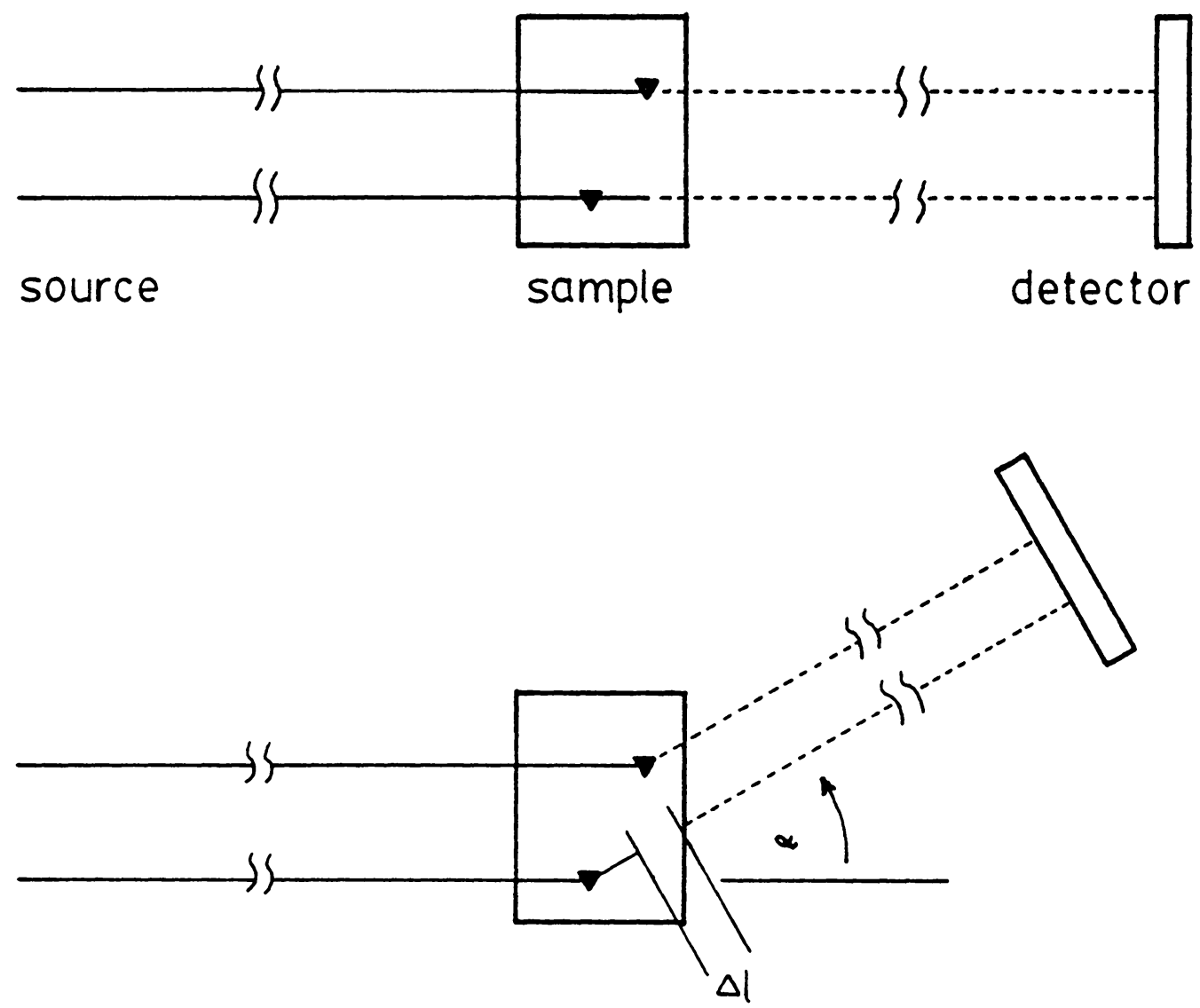

Figure 2.1: The top figure shows that at zero degrees the pathlength of the $x$-ray is the same regardless of the location of the scattering center. When the scattering angle is greater than zero (lower figure) the pathlength is dependent on location of the scattering center. 


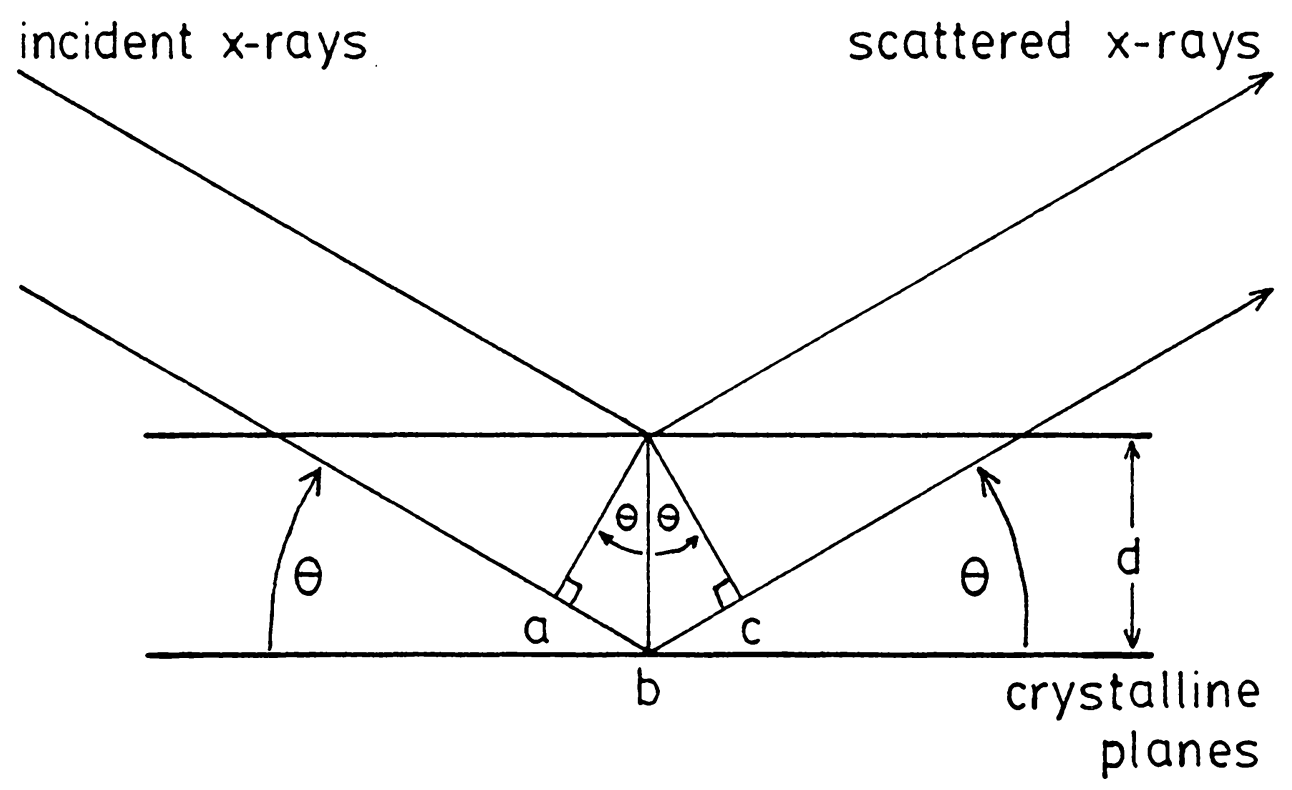

$$
\begin{gathered}
d=\begin{array}{c}
\text { spacing between planes } \\
\delta=\text { path difference } \\
\delta=a b+b c \\
\delta=d \sin \theta+d \sin \theta \\
\delta=2 d \sin \theta
\end{array}
\end{gathered}
$$

constructive interference when $\delta=n \lambda$ $n \lambda=2 d \sin \theta$

Figure 2.2: Derivation of Bragg's law form reflection geometry. 


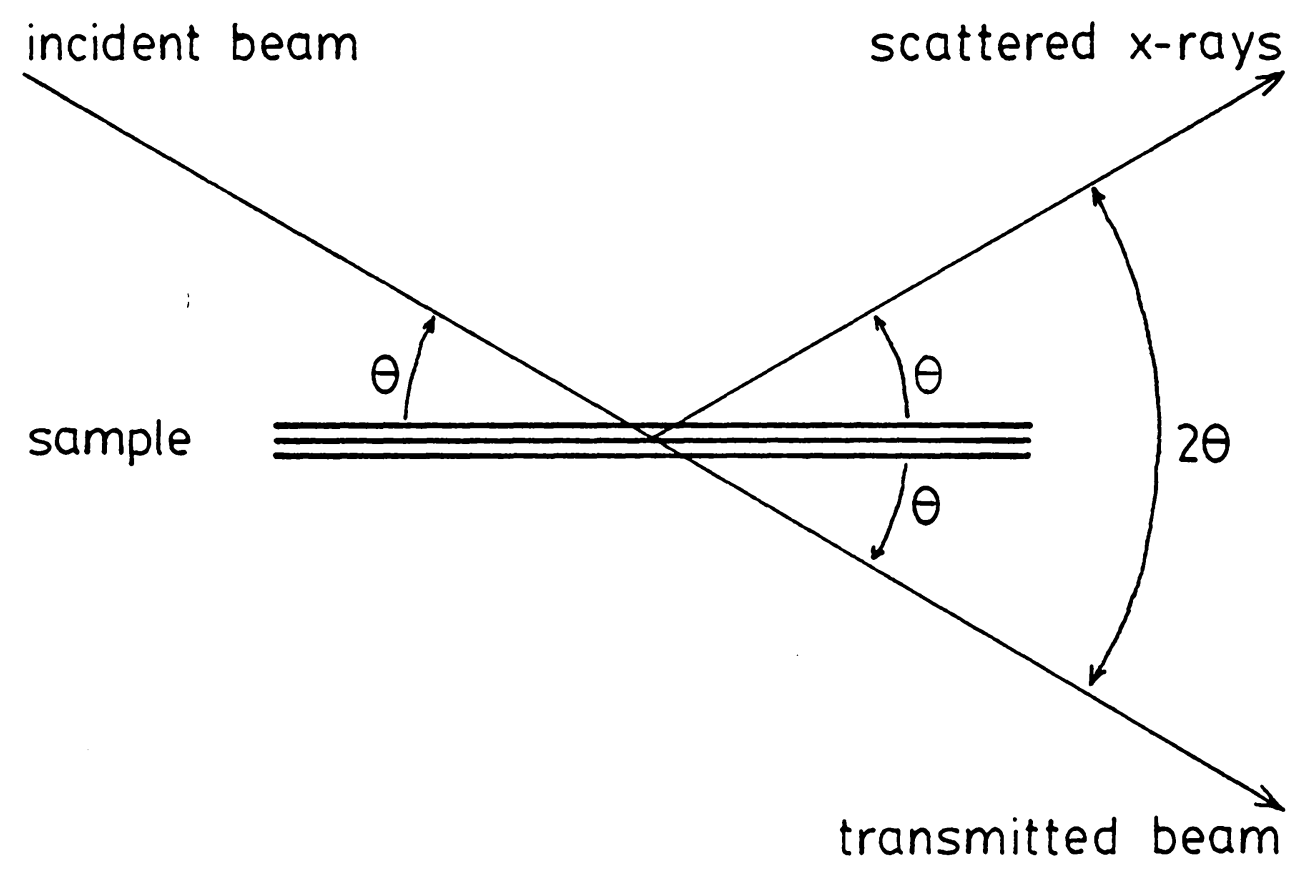

Figure 2.3: Transmission geometry in which measured angle is twice the Bragg angle. 

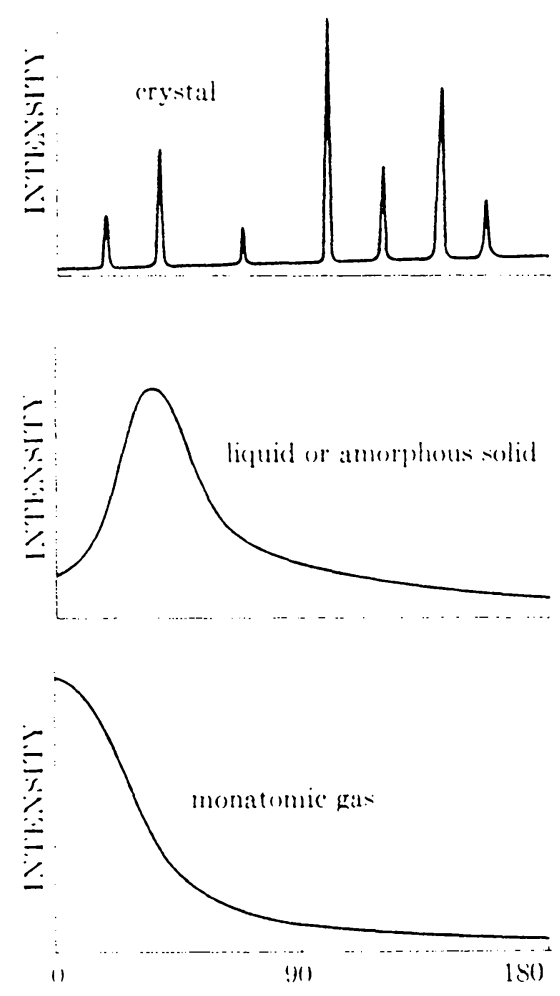

DIFFRA(TION IAT ATTERIN(i)

AN(ill: $2 \theta$ idegrees)

Figure 2.4: Comparative wide angle $x$-ray scattering by crystalline solids, amorphous solids or liquids, and monatomic gases (ref. 32). 


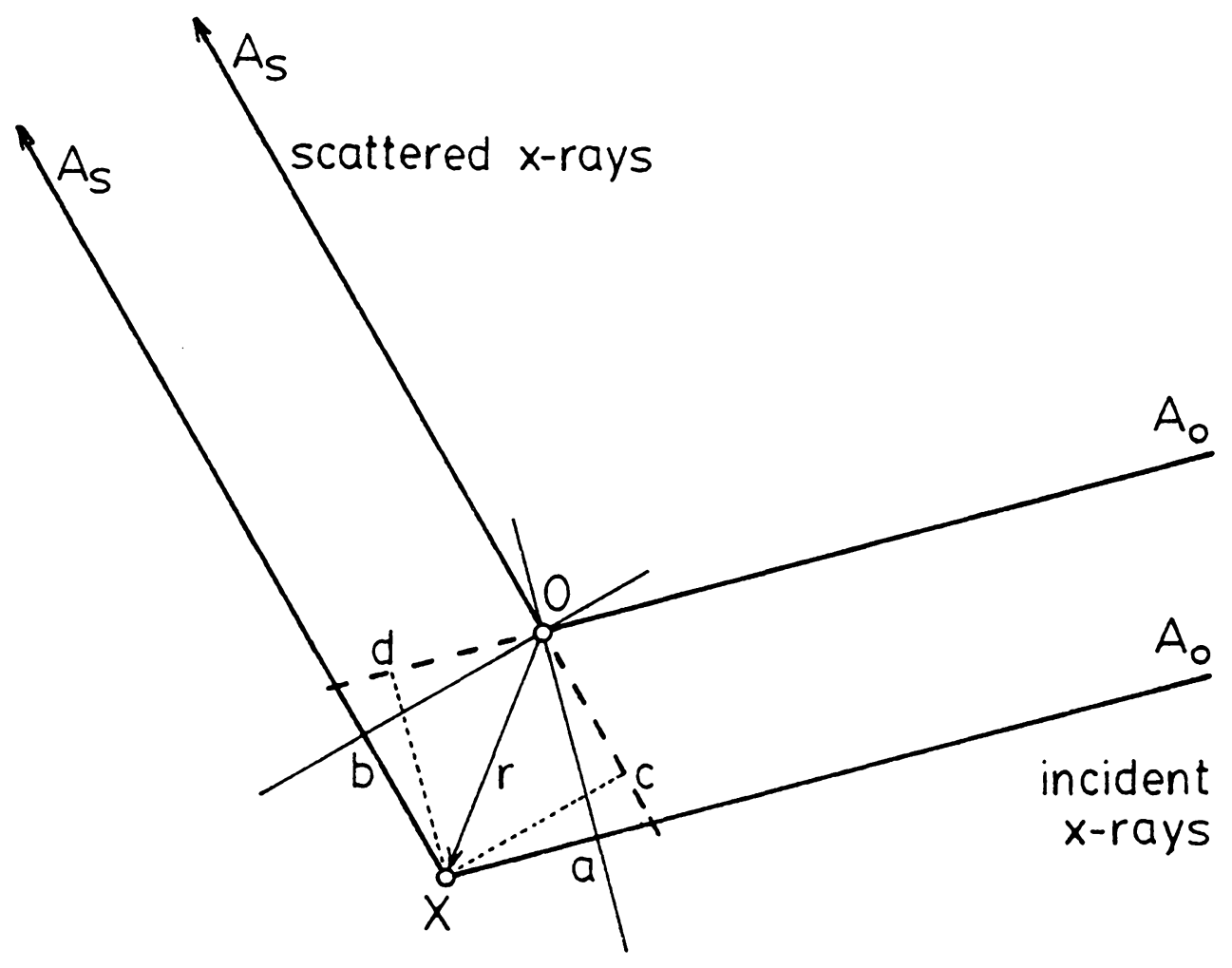

$$
\begin{gathered}
\delta=a X+b X \\
\delta=d O+c O \\
\delta=r \cdot A o-r \cdot(-A s) \\
\delta=-r \bullet(A s-A o)
\end{gathered}
$$

Figure 2.5: Determination of path difference in terms of incident and scattered directions and the vector $r$ between them. 


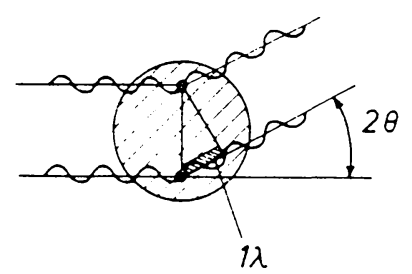

(a)

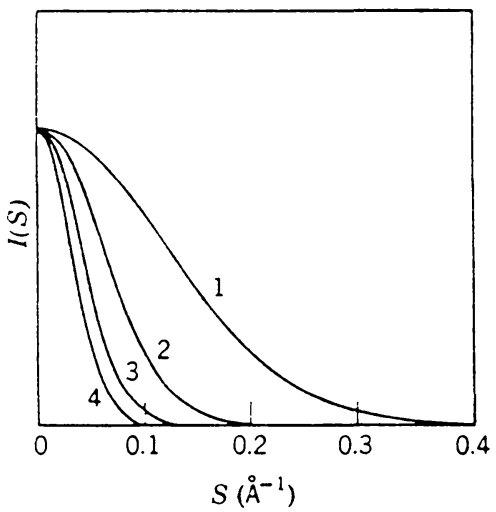

(a)

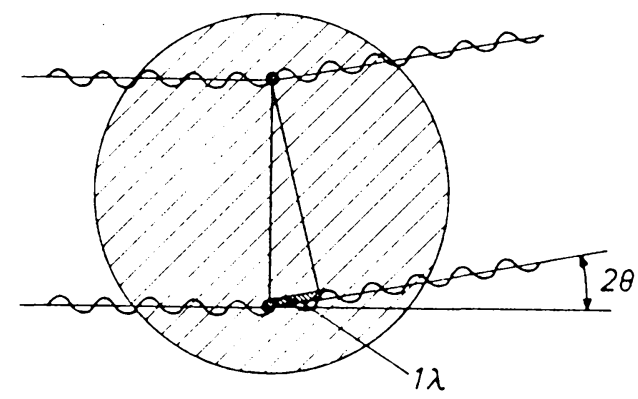

(b)

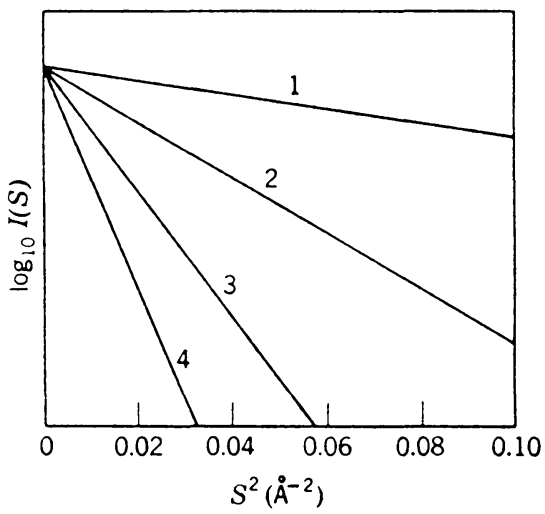

(b)

Figure 2.6: The top figure illustrates that larger particles scatter at lower angles than smaller particles (ref. 35). The lower figure shows small angle curves conforming to the law of Guinier. Particle sizes are in the ratio 1 : $2: 3: 4$ (ref. 33 ). 

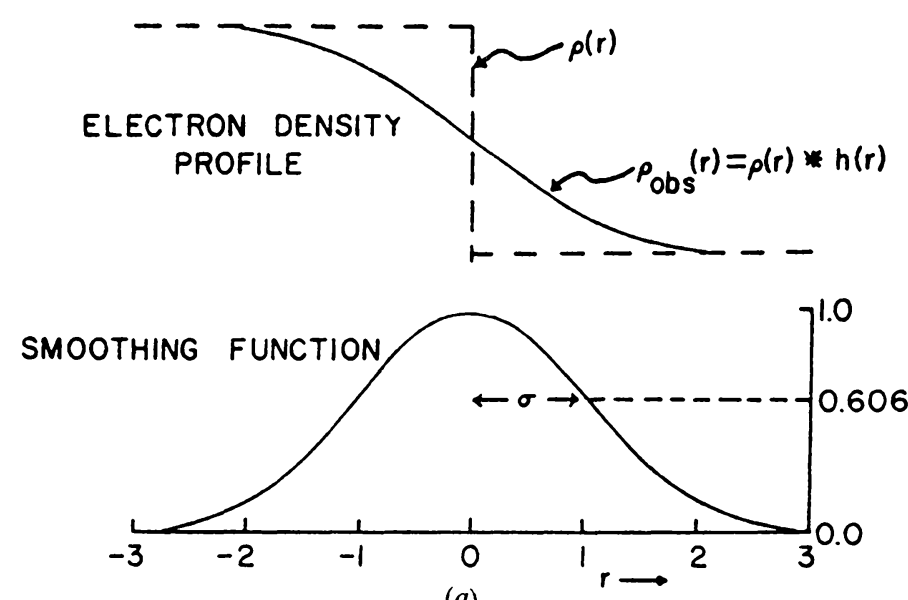

(a)

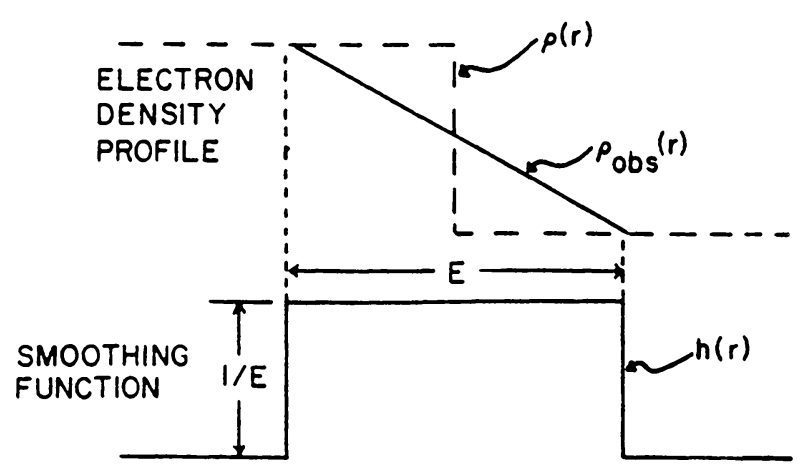

Figure 2.7: Relationship of $\sigma$ to the diffuse boundary thickness and comparison of sigmoidal gradient to linear gradient (ref. 36). 
a.

$\overline{\Delta \rho^{2}}$

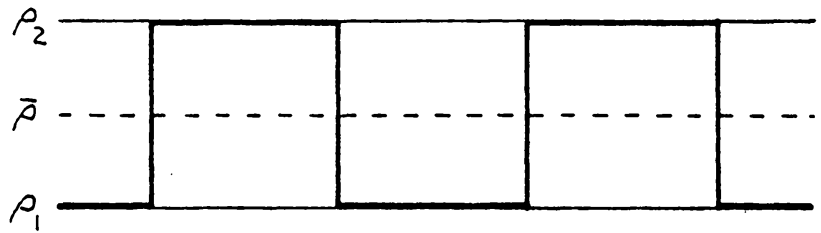

b.

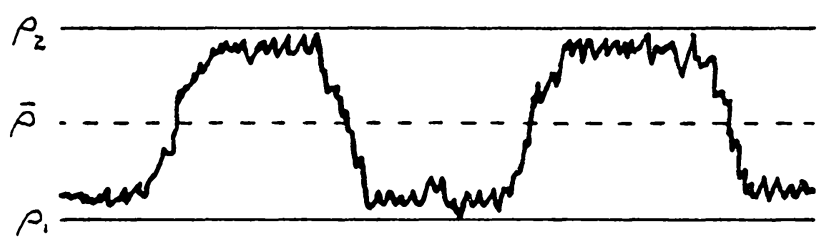

C.
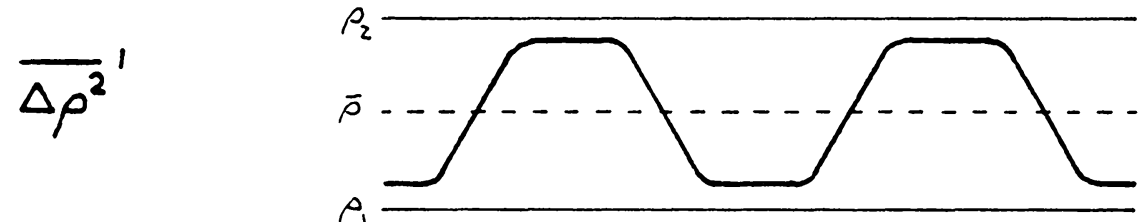

d. $\quad \frac{}{\Delta p^{2}}$ "

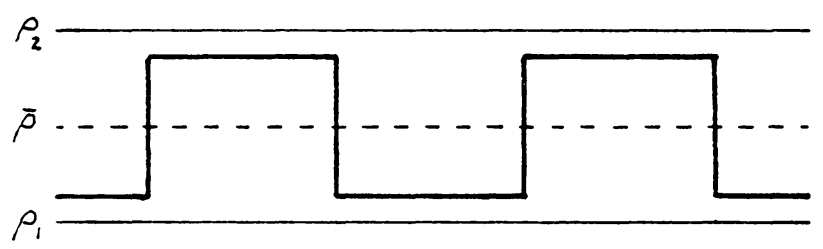

Figure 2.8: Electron density profiles for a) completely phase separated system with sharp phase boundaries b) real system with phase mixing, background, and diffuse boundaries c) corrected for background d) corrected for background and diffuse boundaries. 


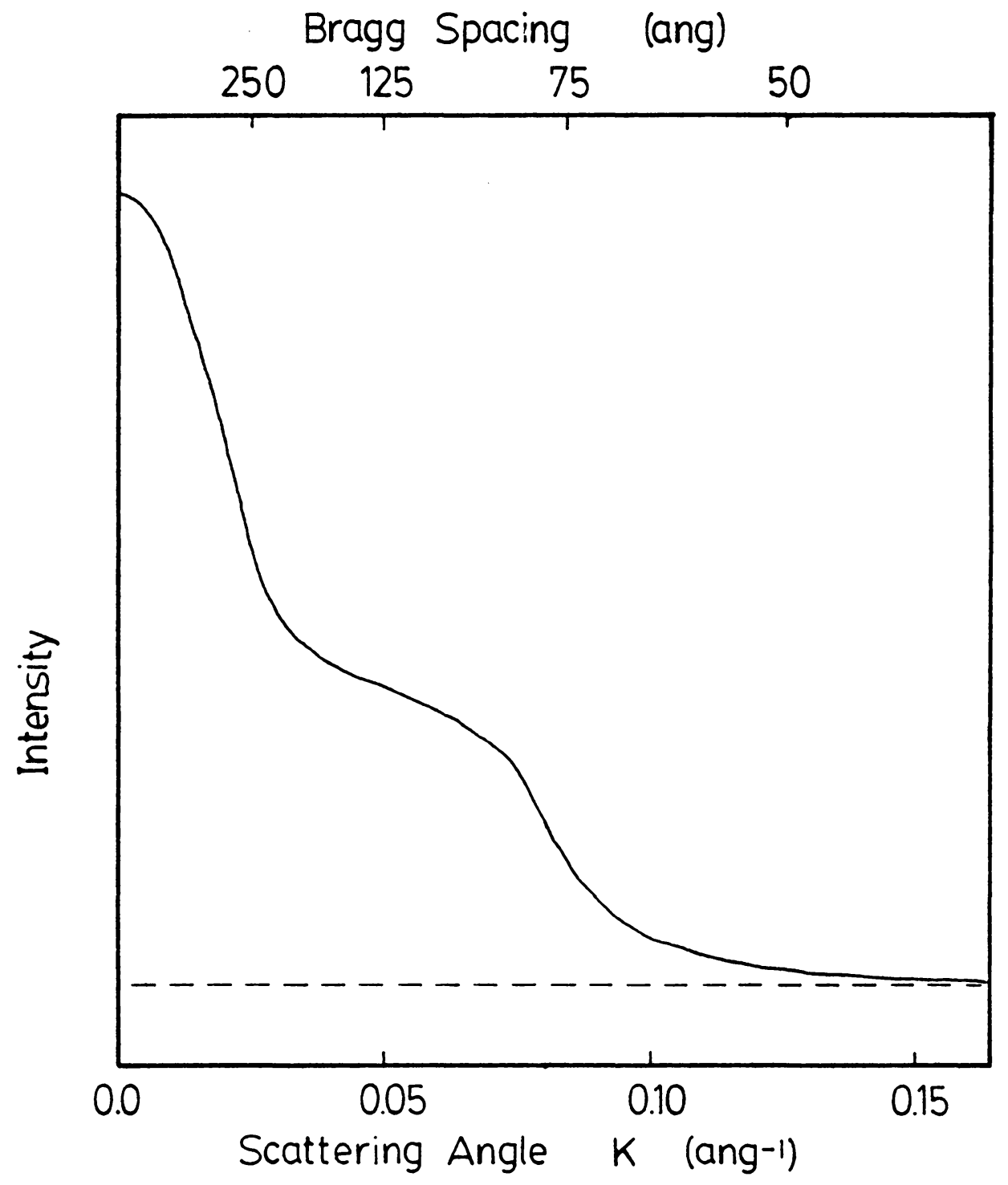

Figure 2.9: Hypothetical scattering profile for a phase separated segmented copolymer. 
a) PPO repeat unit
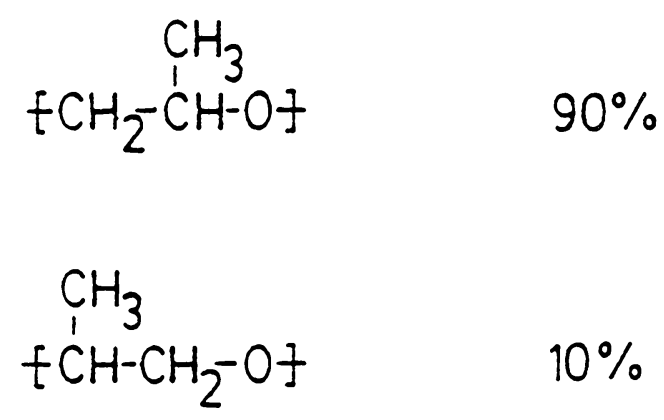

b) Polyurea repeat unit

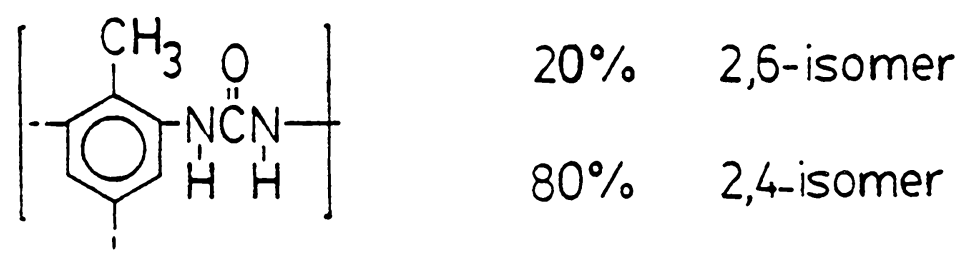

Figure 4.1: Repeat units for PPO soft segment and a polyurea hard segment based on water extended TDI-80. 


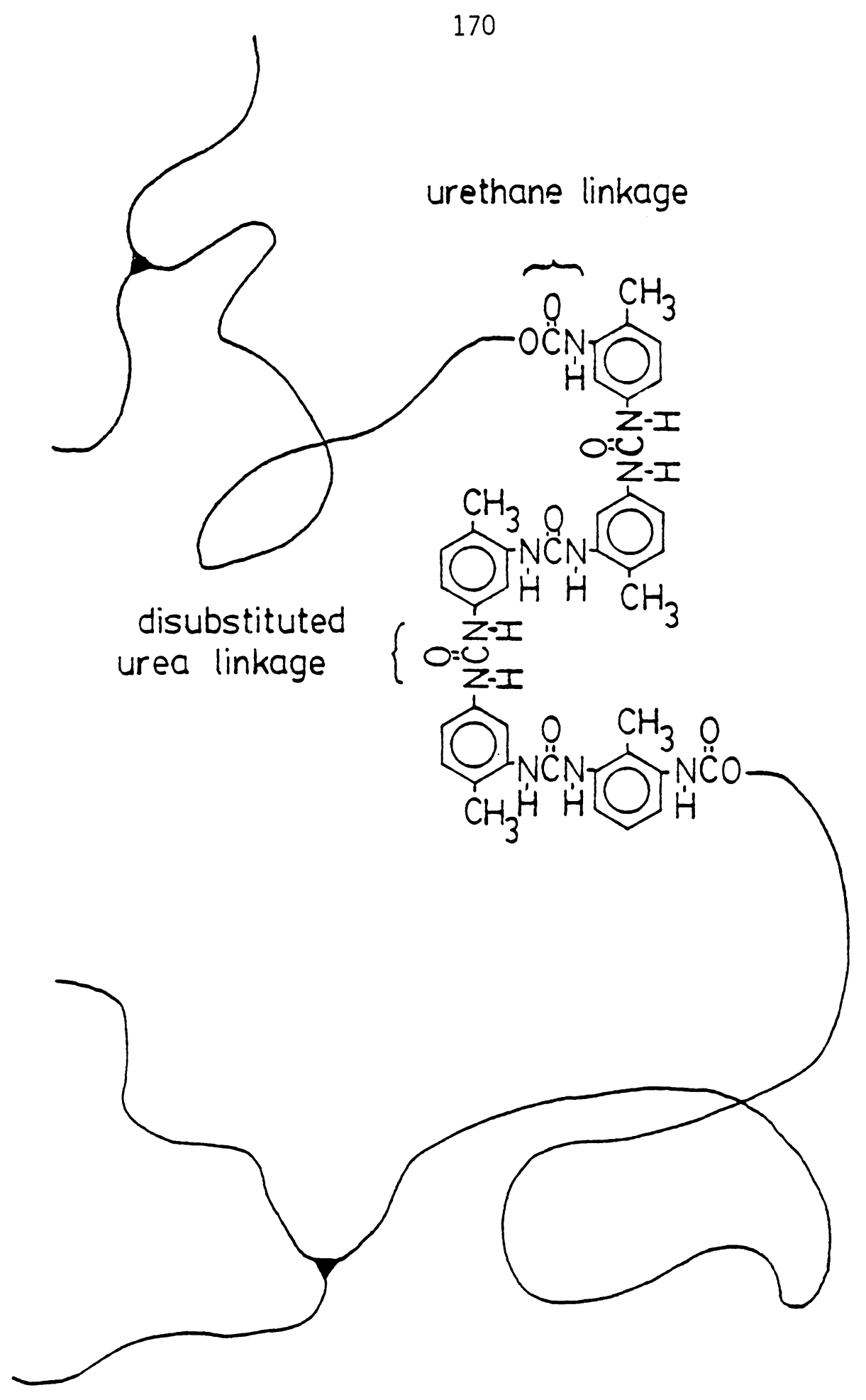

Figure 4.2: Structure of a network chain in a water-blown polyurethane foam. 

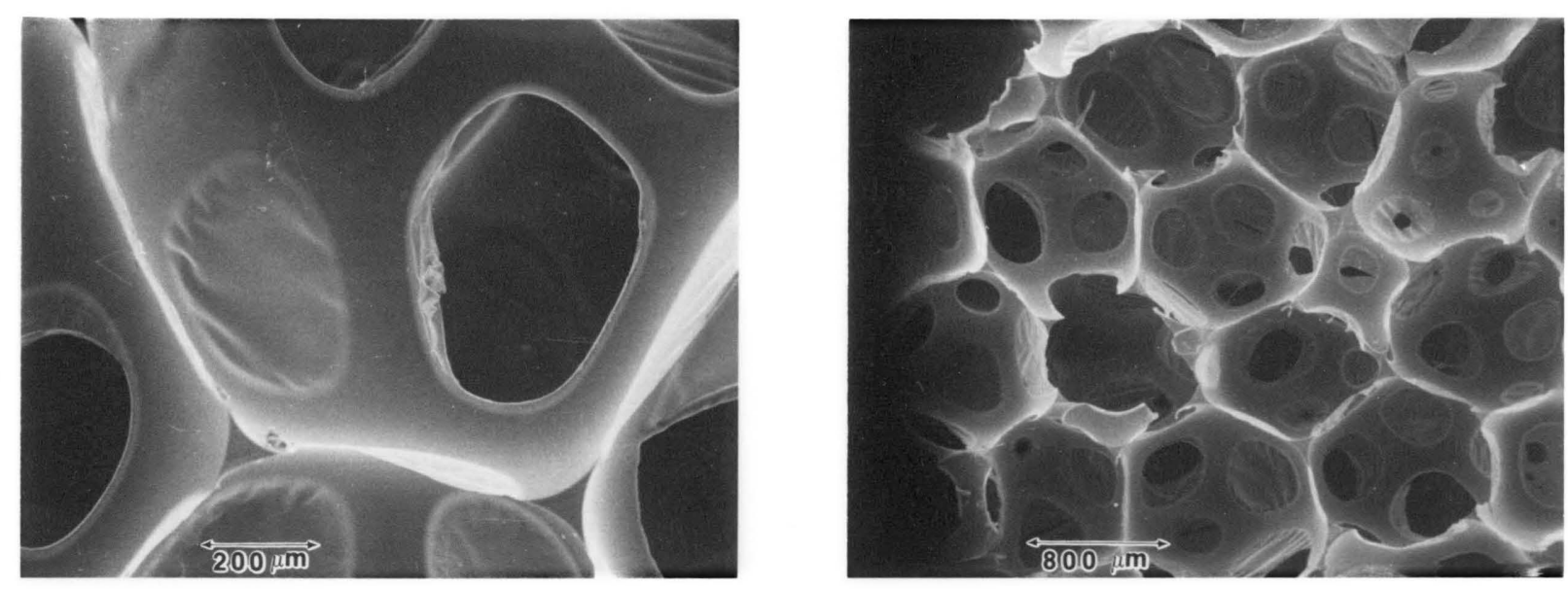

blow direction
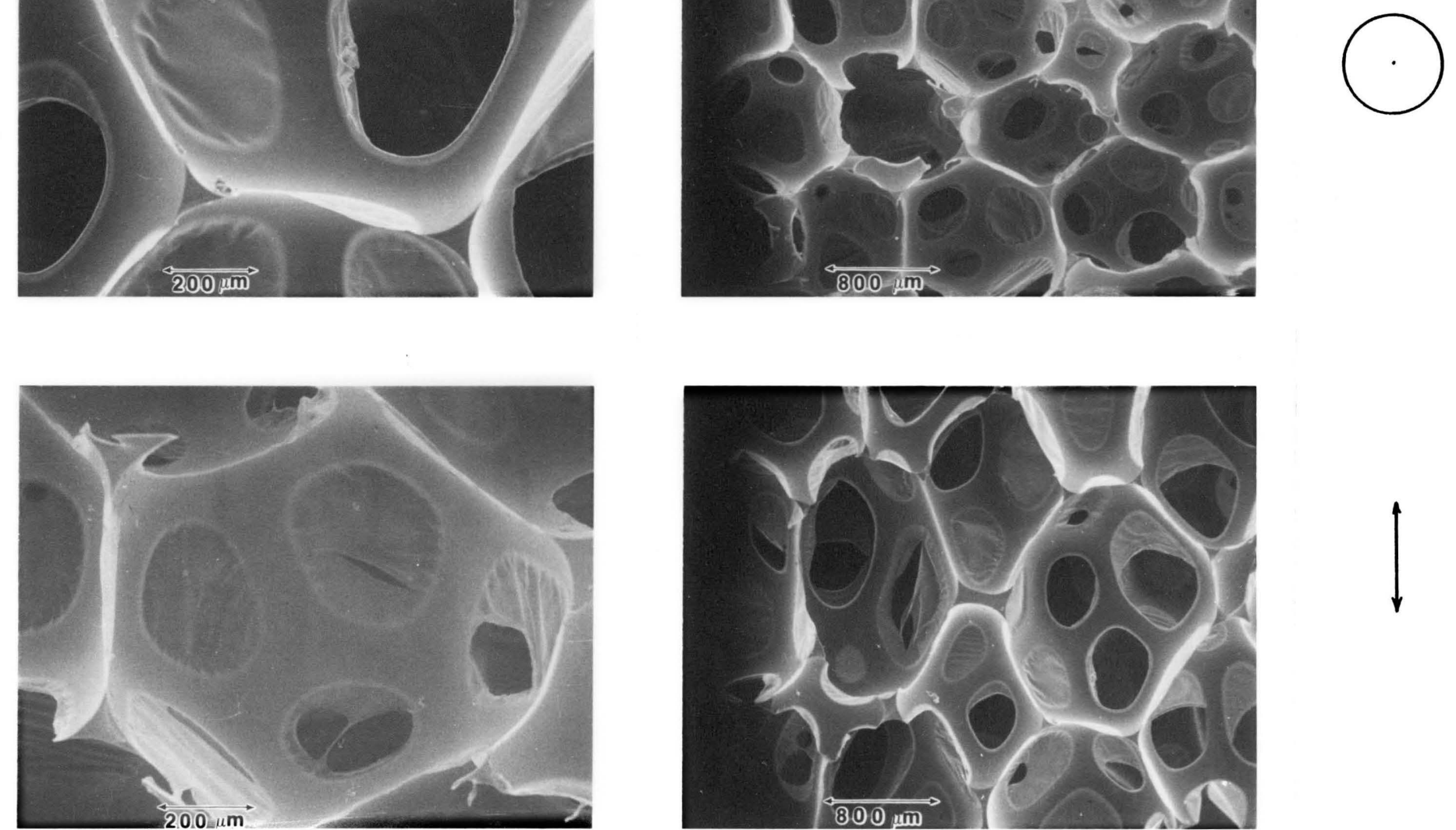

Figure 4.3: $\quad$ SEM micrographs of foam 31135-33-1. 

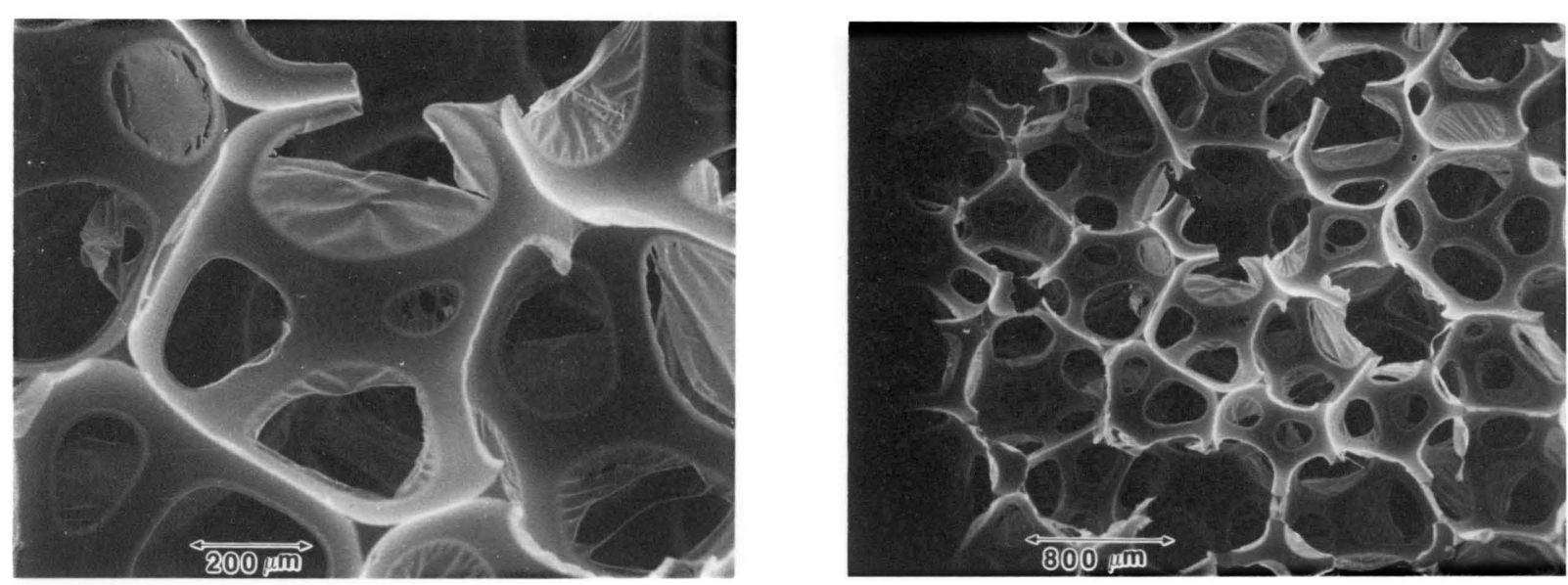

\section{blow direction}
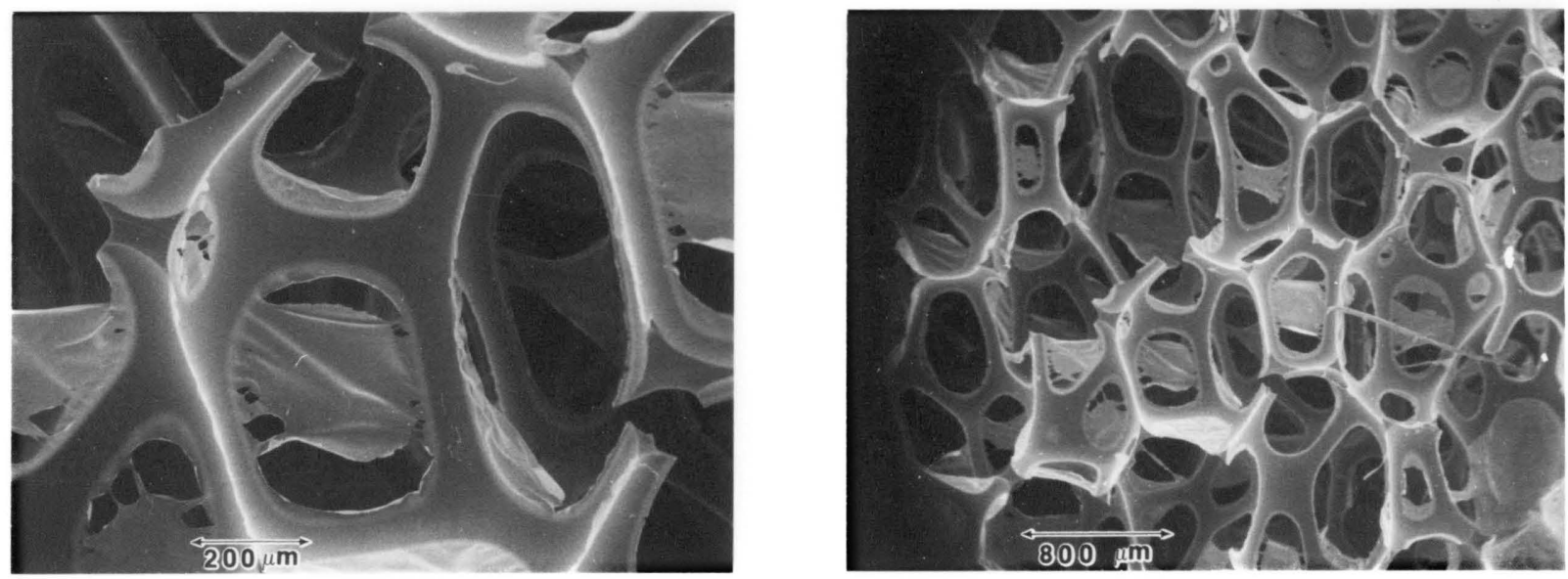

Figure 4.4: SEM micrographs of foam 31135-33-2. 

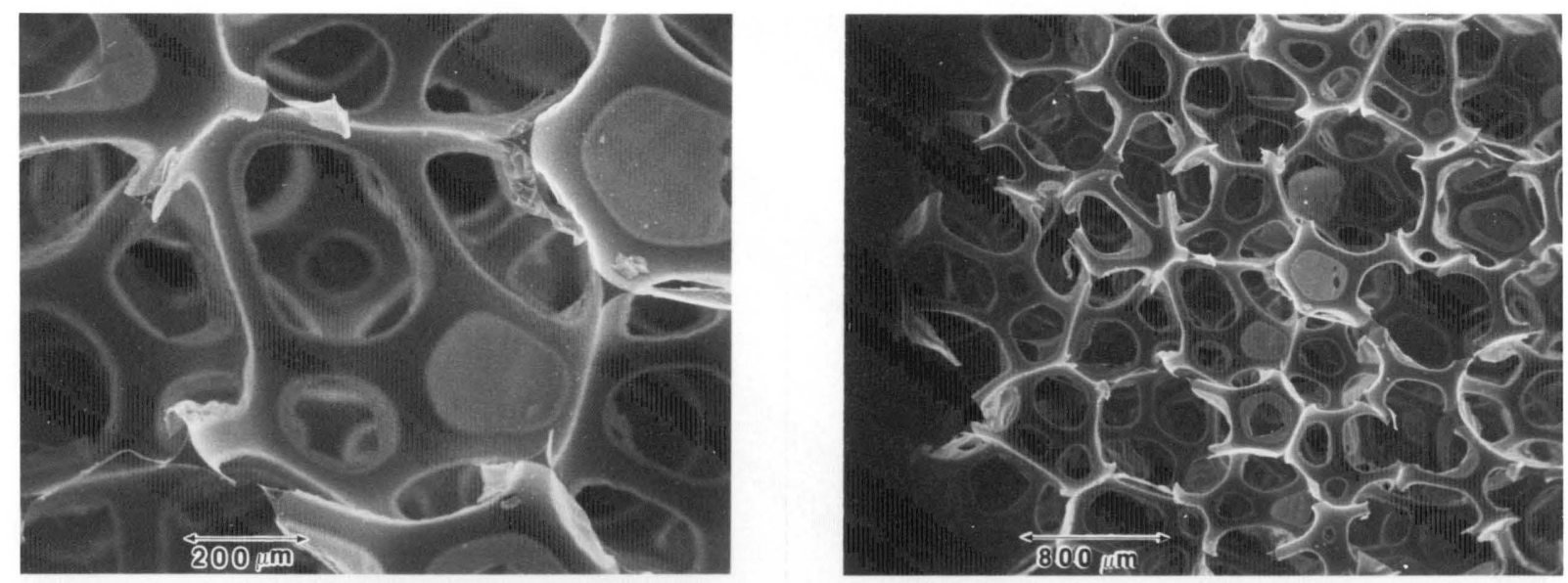

blow

direction
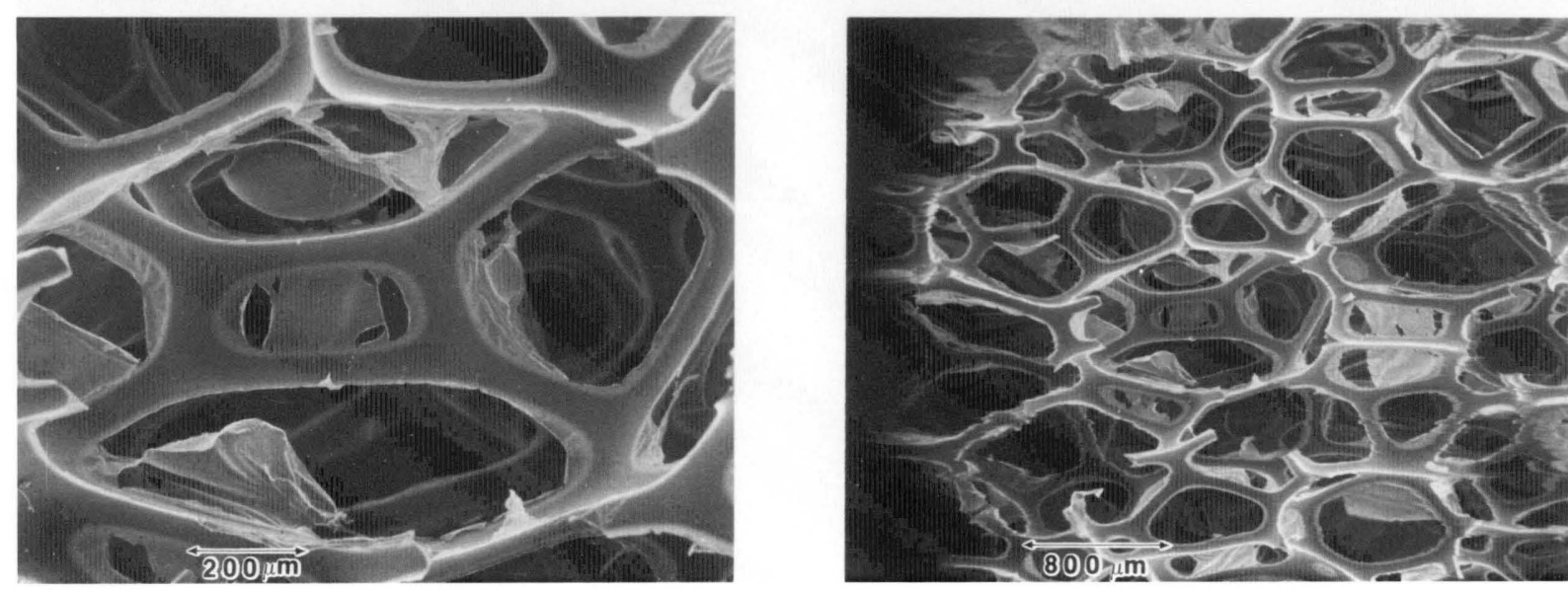

Figure 4.5: $\quad$ SEM micrographs of foam 31135-33-3. 

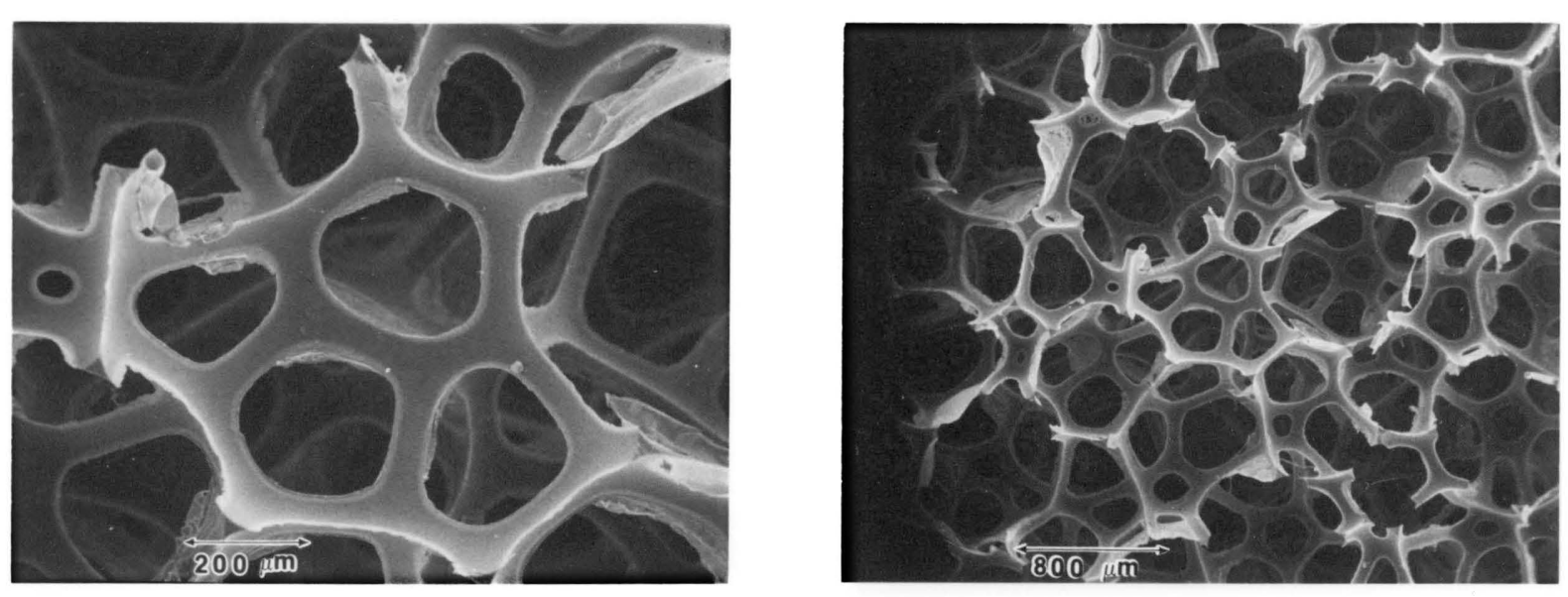

blow direction
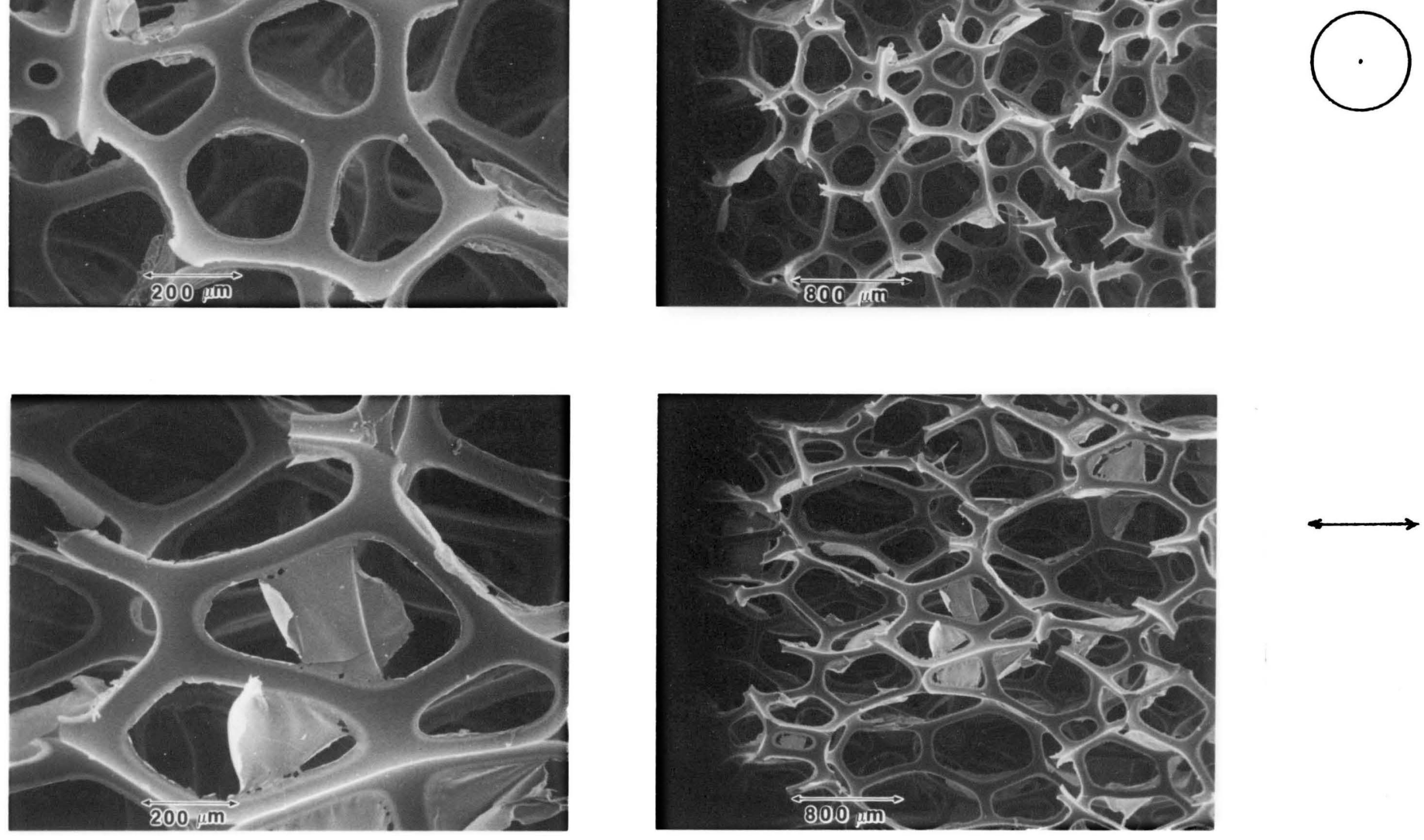

$\stackrel{\Xi}{\triangleright}$

Figure 4.6: SEM micrographs of foam 31135-33-4. 

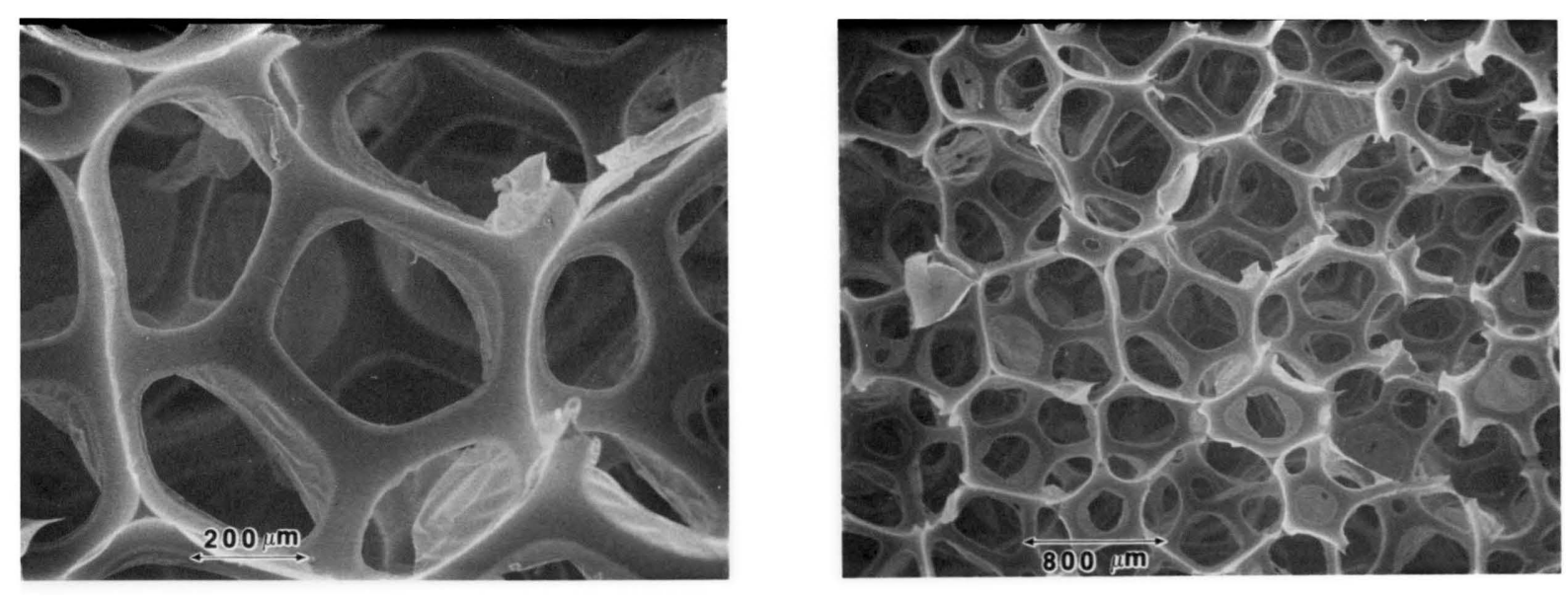

blow direction
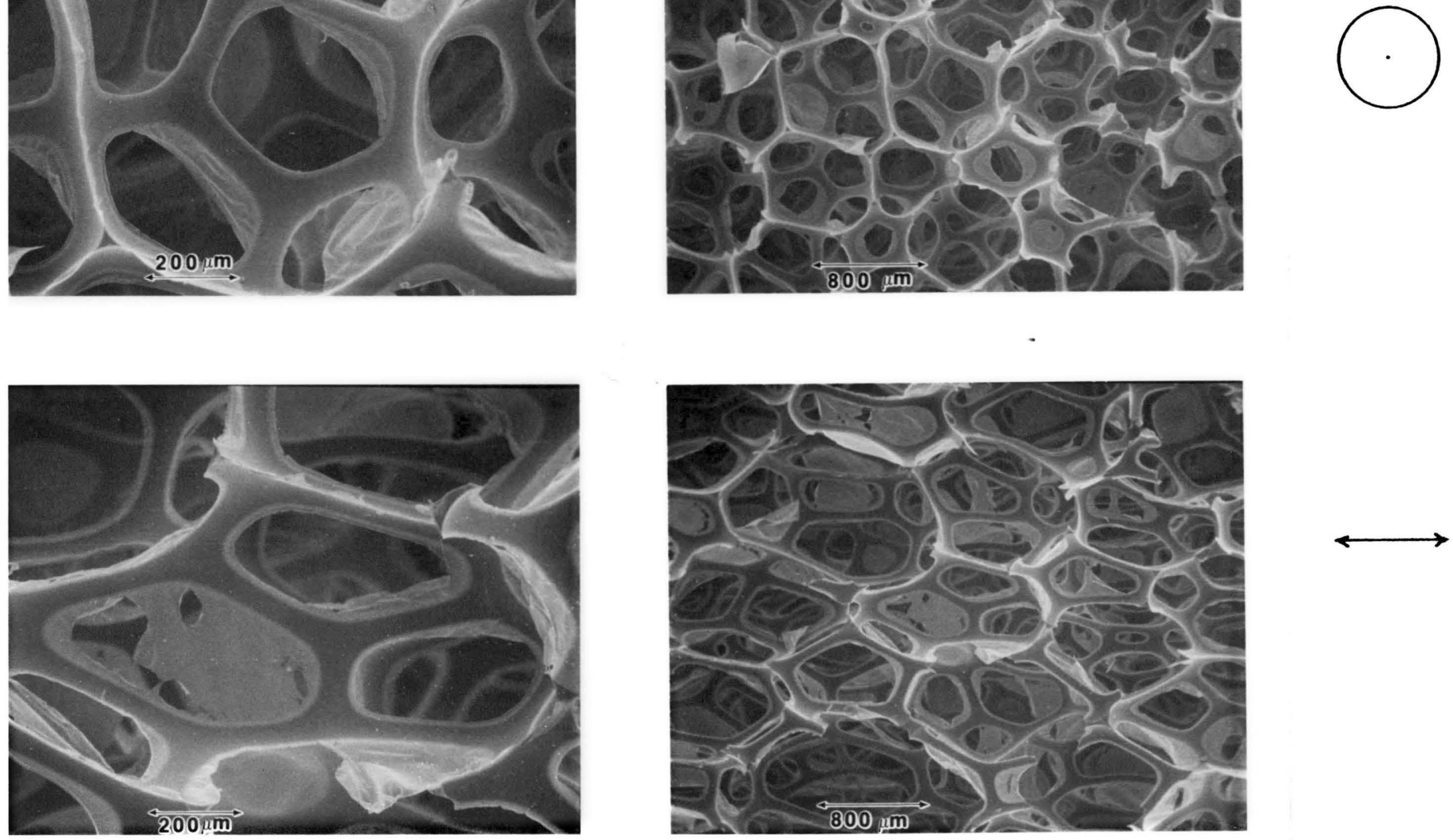

Figure 4.7: SEM micrographs of foam 31135-33-5. 

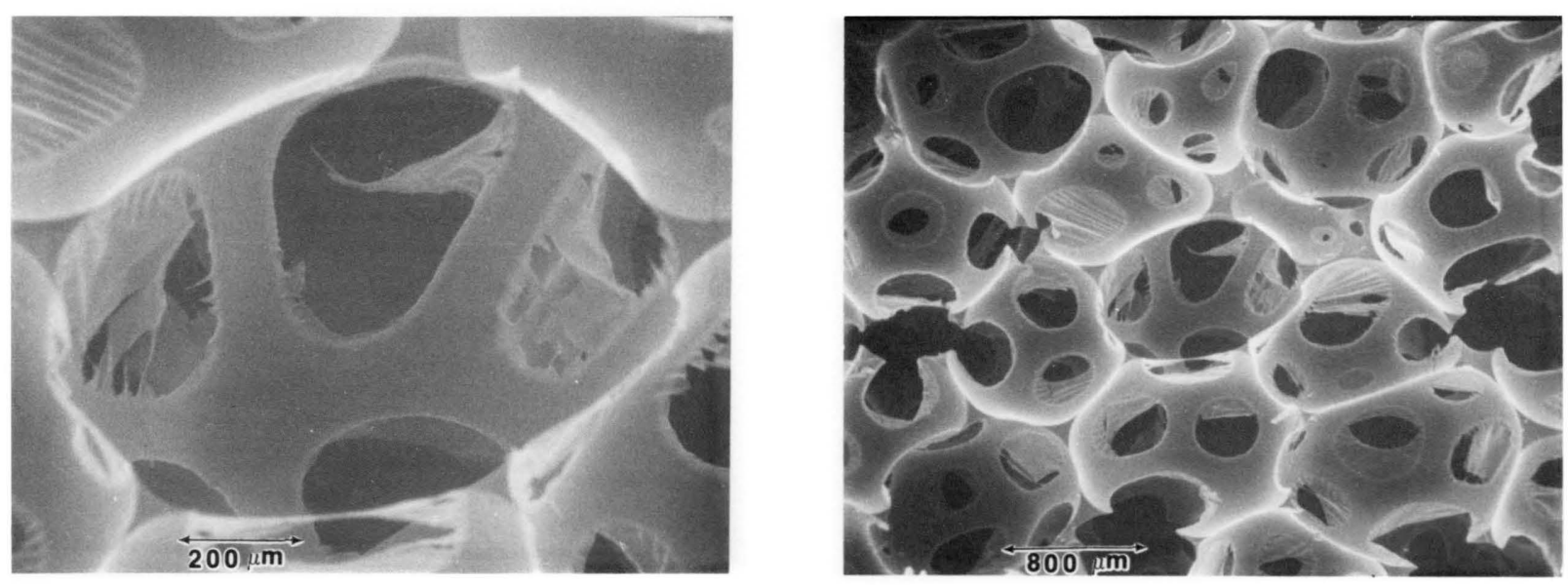

\section{blow \\ direction}
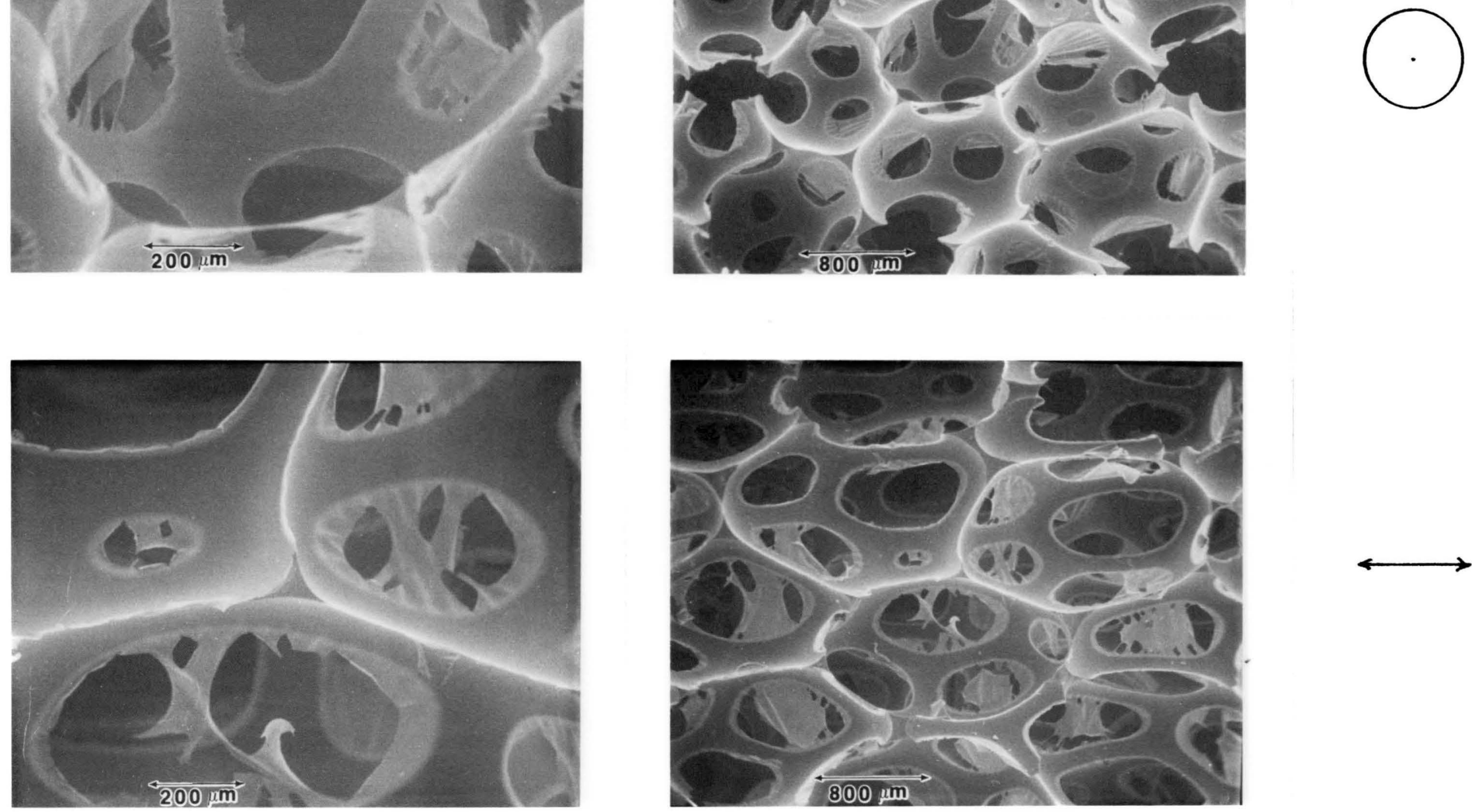

Figure 4.8: SEM micrographs of foam 31135-33-6. 


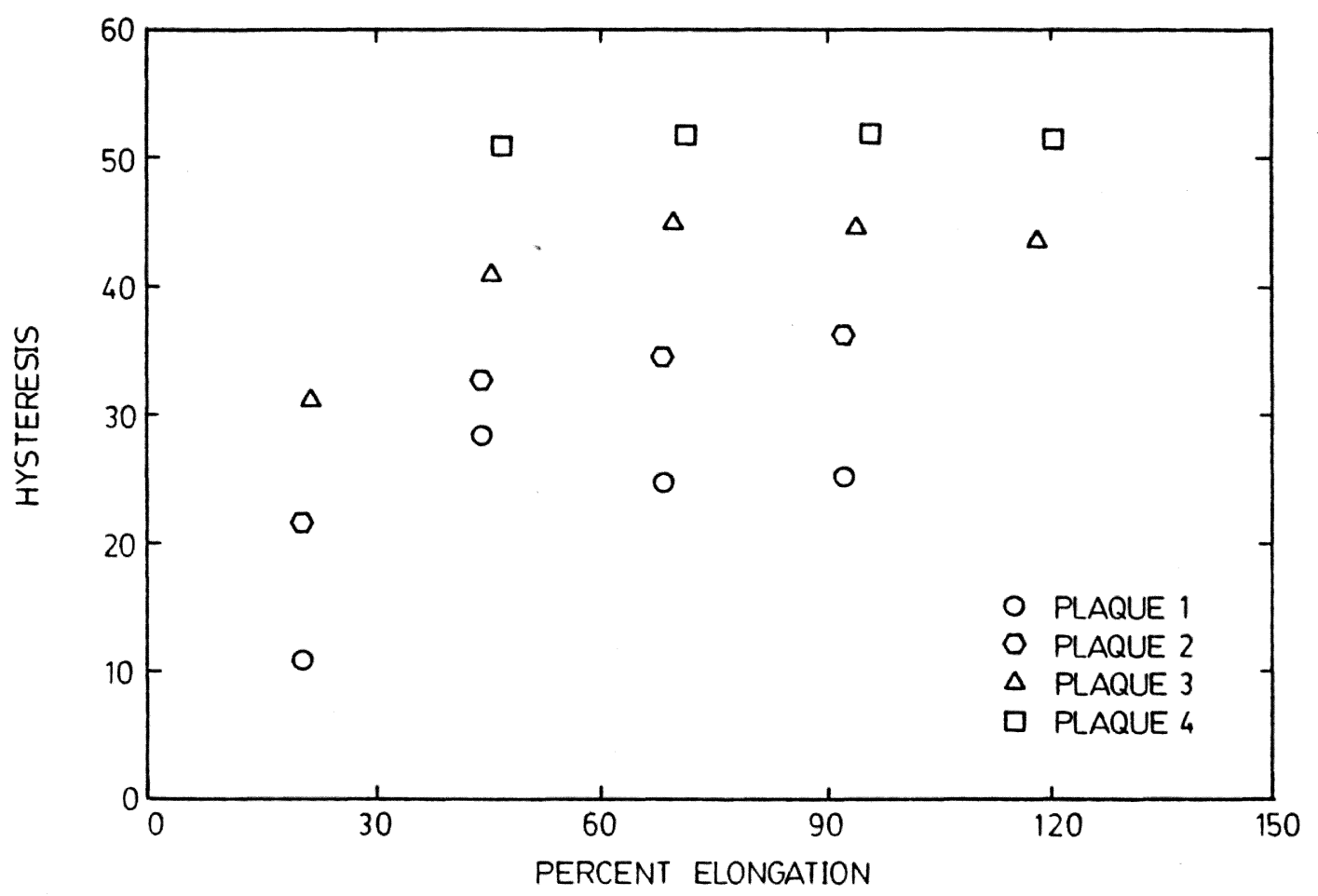

Figure 4.9: Percent hysteresis for plaques 1 through 4. 


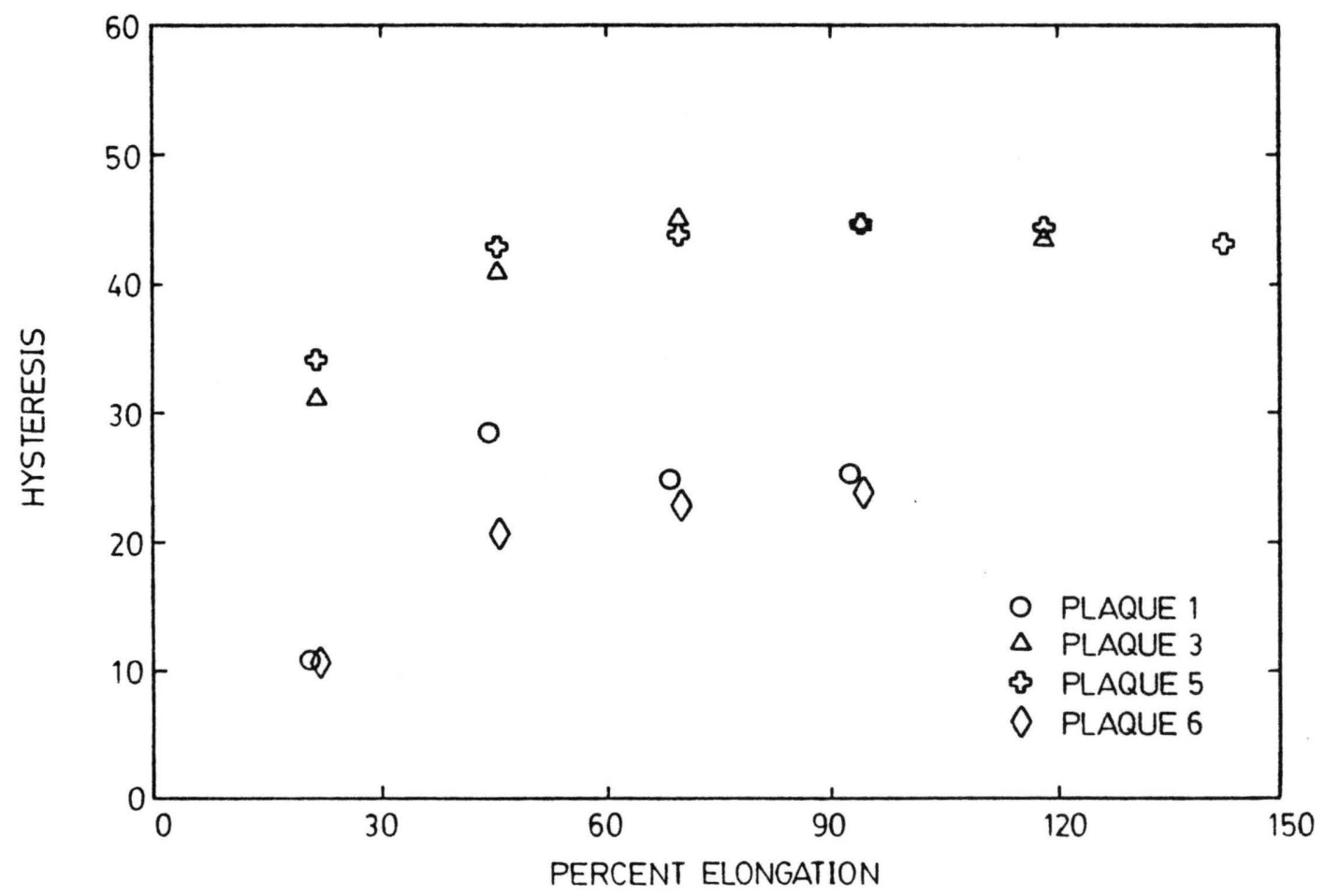

Figure 4.10: Percent hysteresis for plaque pairs 1,6 and 3,5. Each member of a pair was made with the same water content but different tin catalyst levels. 


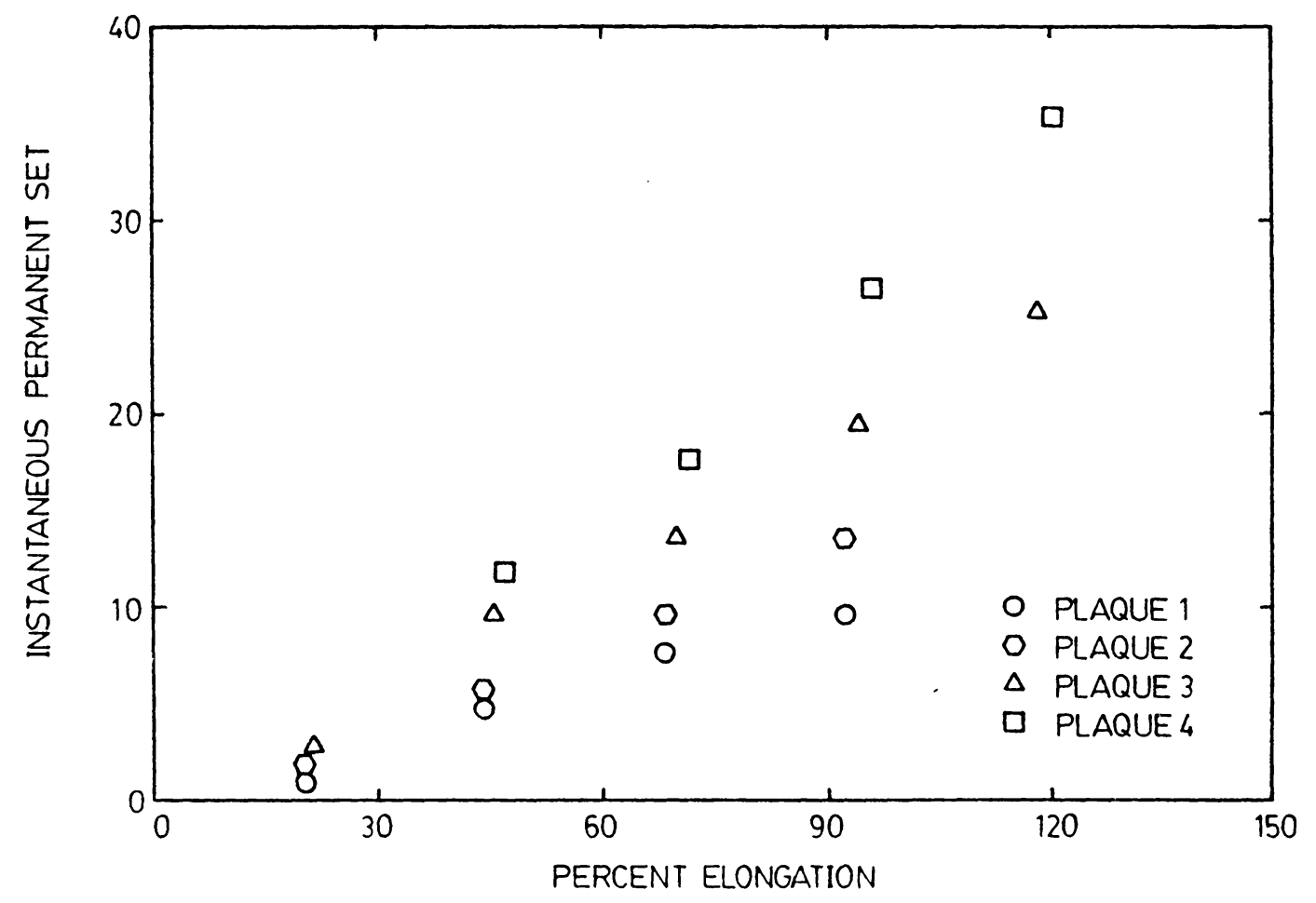

Figure 4.11: Percent "Instantaneous" permanent set for plaques 1 through 4 . 


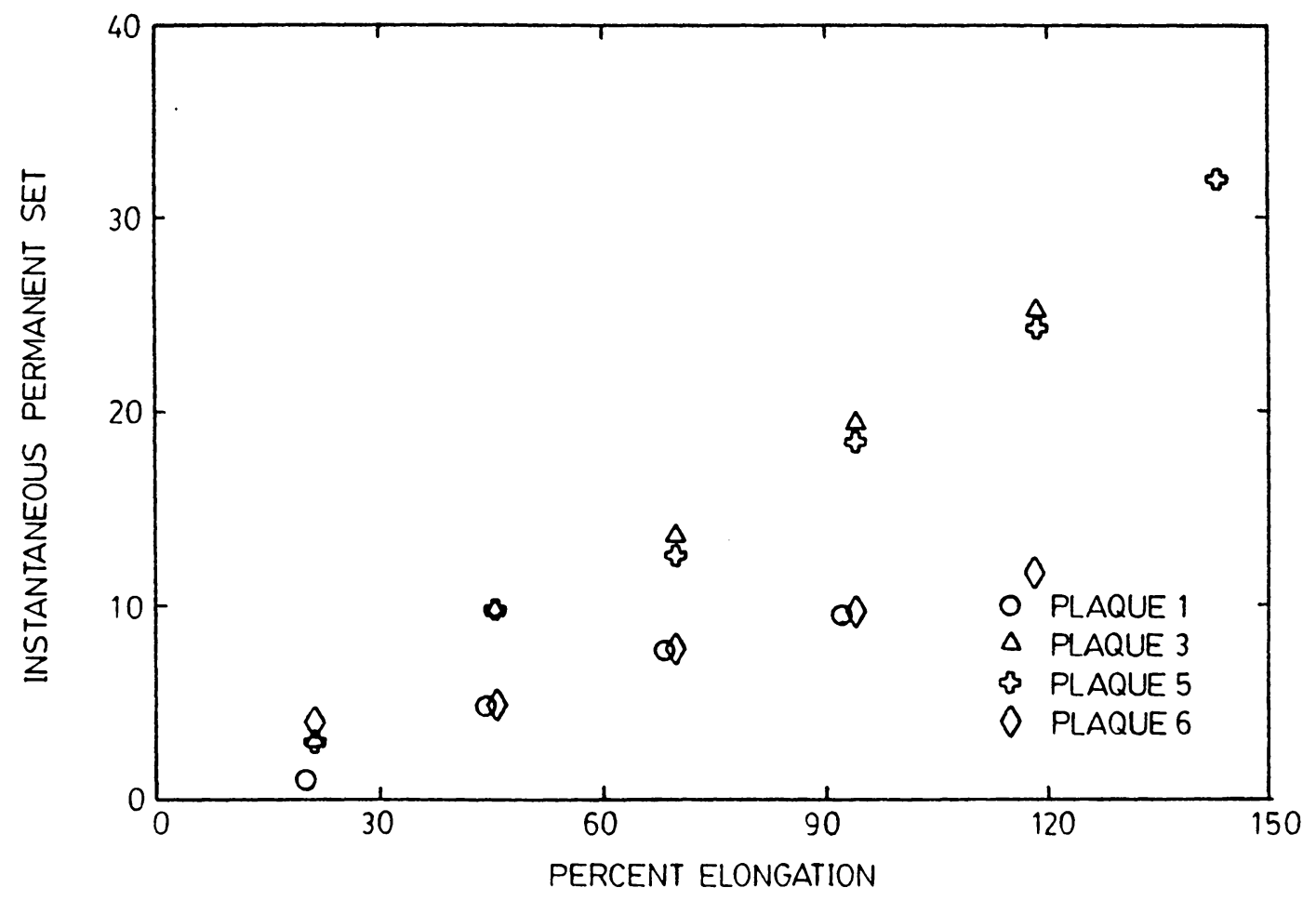

Figure 4.12: Percent "Instantaneous" permanent set for plaque pairs 1,6 and 3,5. Each member of a pair was made at the same water content but with different tin catalyst concentration. 


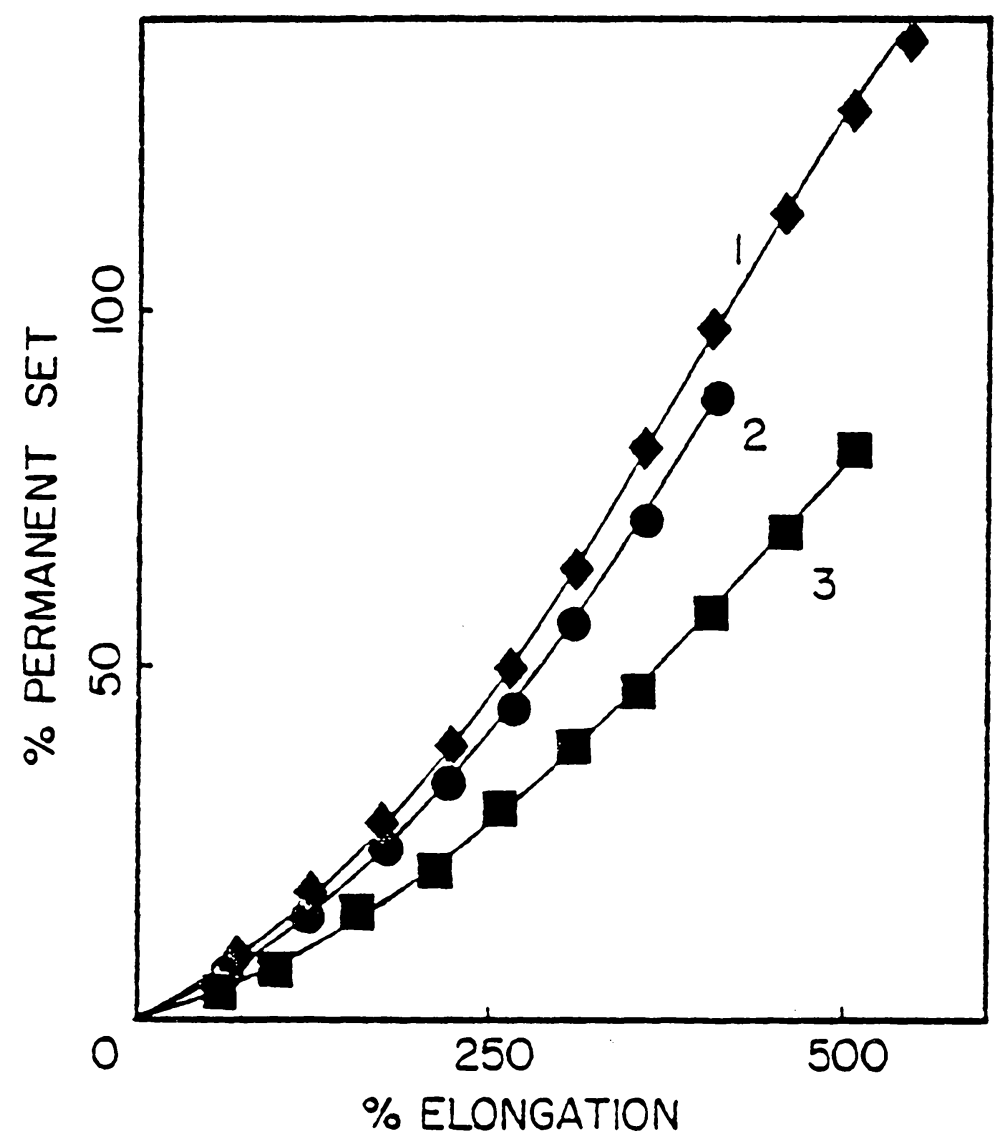

Figure 4.13: Curve 3 is the percent "instantaneous" permanent set for a polyurethane-urea elastomer based on water extended TDI-80 (ref. 28). 

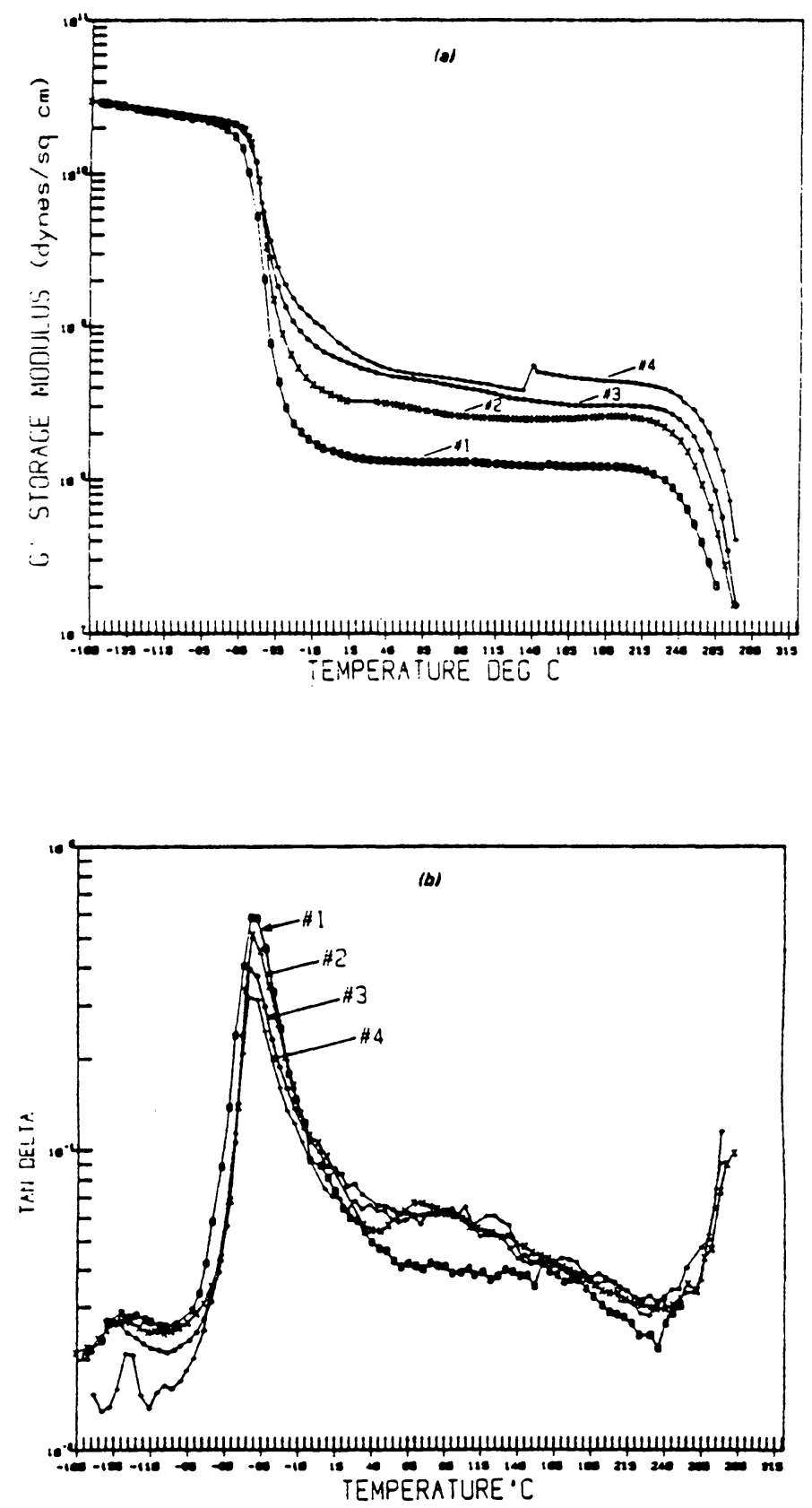

Figure 4.14: Storage modulus and tan delta for foams 1 through 4 . Storage modulii are normalized at $-160 \mathrm{C}$ to the level of a polymeric glass (ref. 81). 


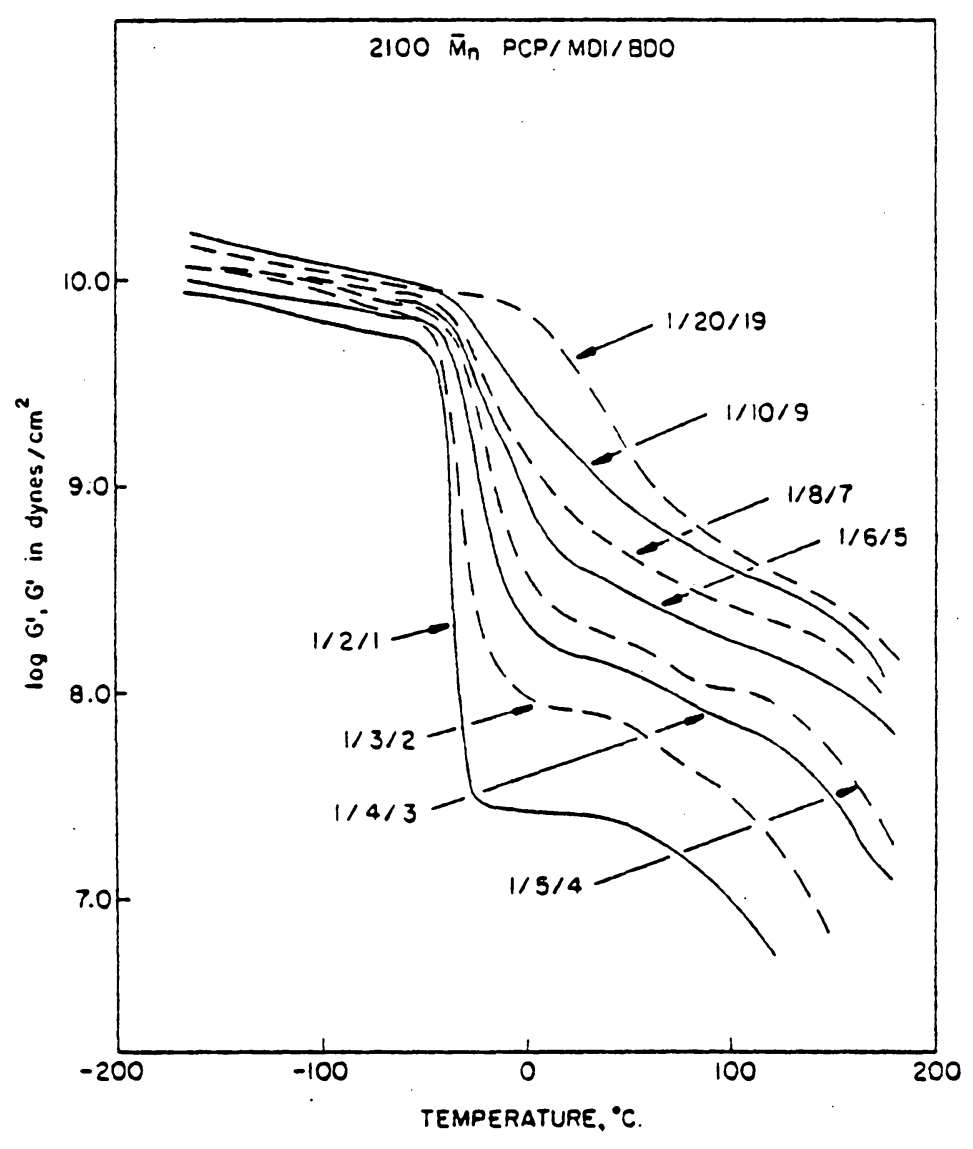

$\begin{array}{lclc}\text { Sample } & \begin{array}{c}\text { MDI-BD } \\ w t \%\end{array} & \text { Sample } & \text { MDI-BD } \\ & 22 & 1 / 6 / 5 & \text { wt } \% \\ 1 / 2 / 1 & 31 & 1 / 8 / 7 & 49 \\ 1 / 3 / 2 & 38 & 1 / 10 / 9 & 56 \\ 1 / 4 / 3 & 43 & 1 / 20 / 19 & 61 \\ 1 / 5 / 4 & & & 76\end{array}$

Figure 4.15: Effect of hard segment content on storage modulus properties of MDI-BD urethane polymers with $2100 \mathrm{MW}$ polycaprolactone diol (ref. 53). 


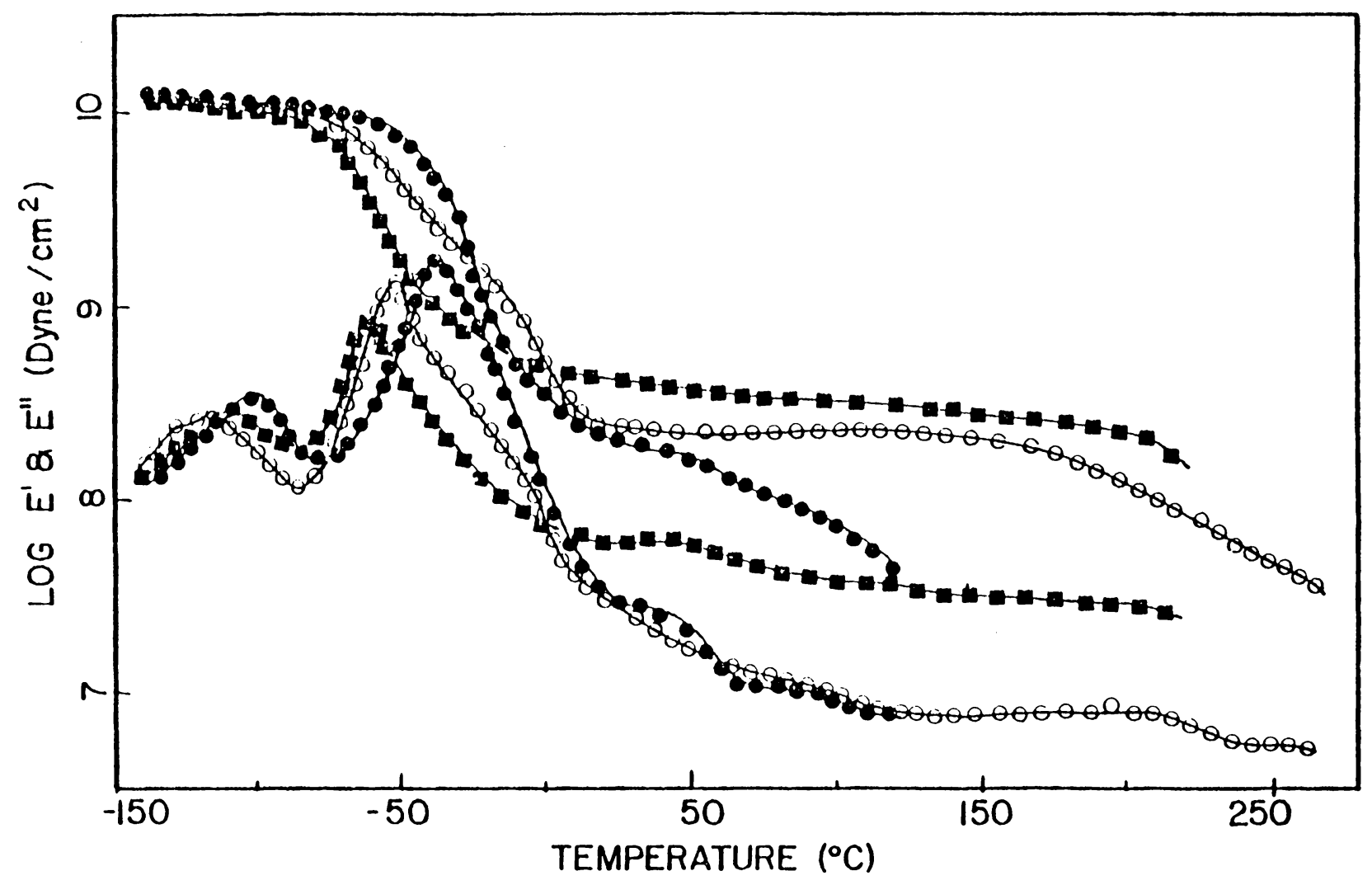

Figure 4.16: The solid squares are the storage and loss moduli of a polyurethane-urea with water extended TDI-80 hard segment (ref 28). 

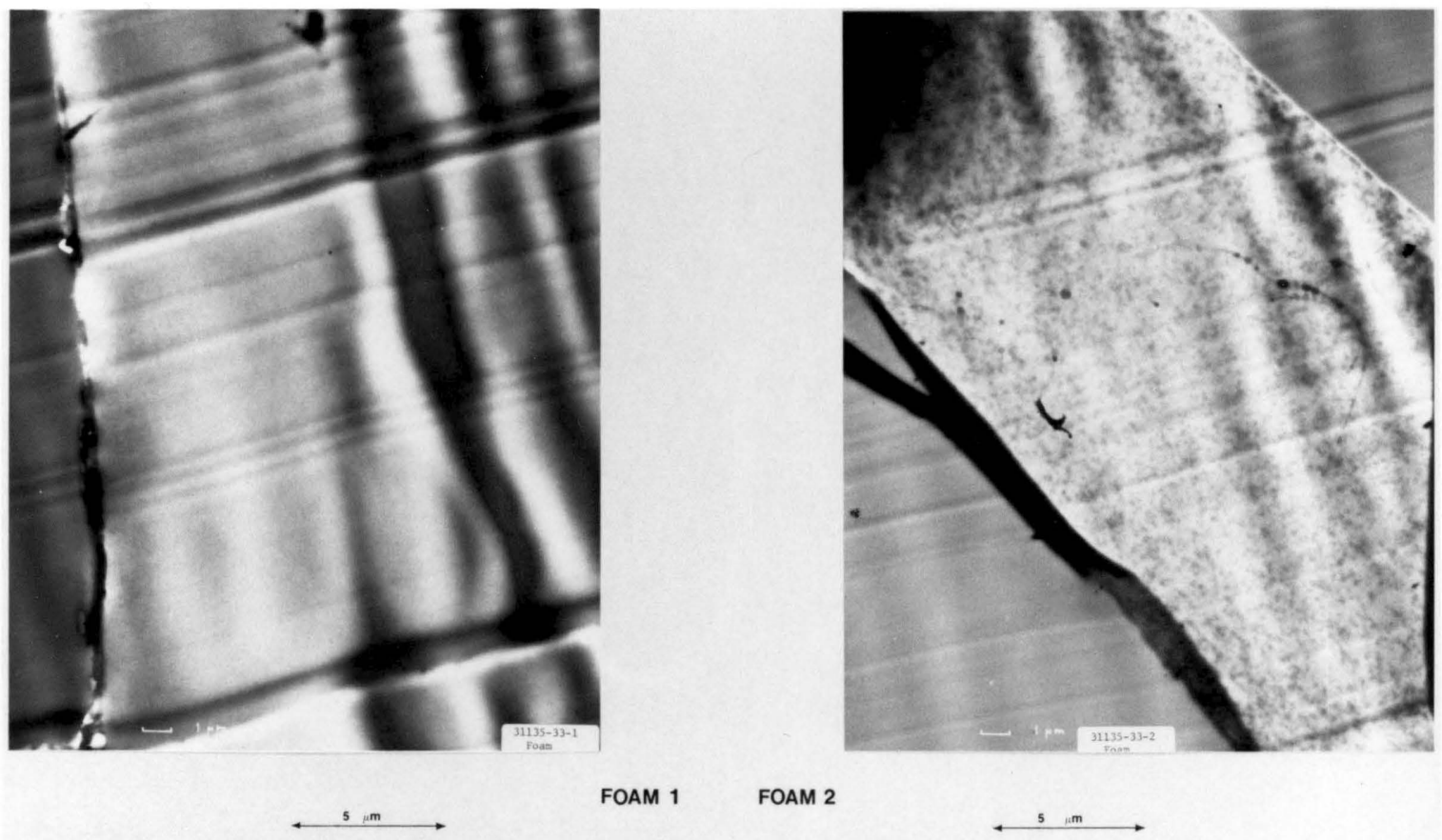

FOAM 1 FOAM 2

FOAM $3 \quad$ FOAM 4
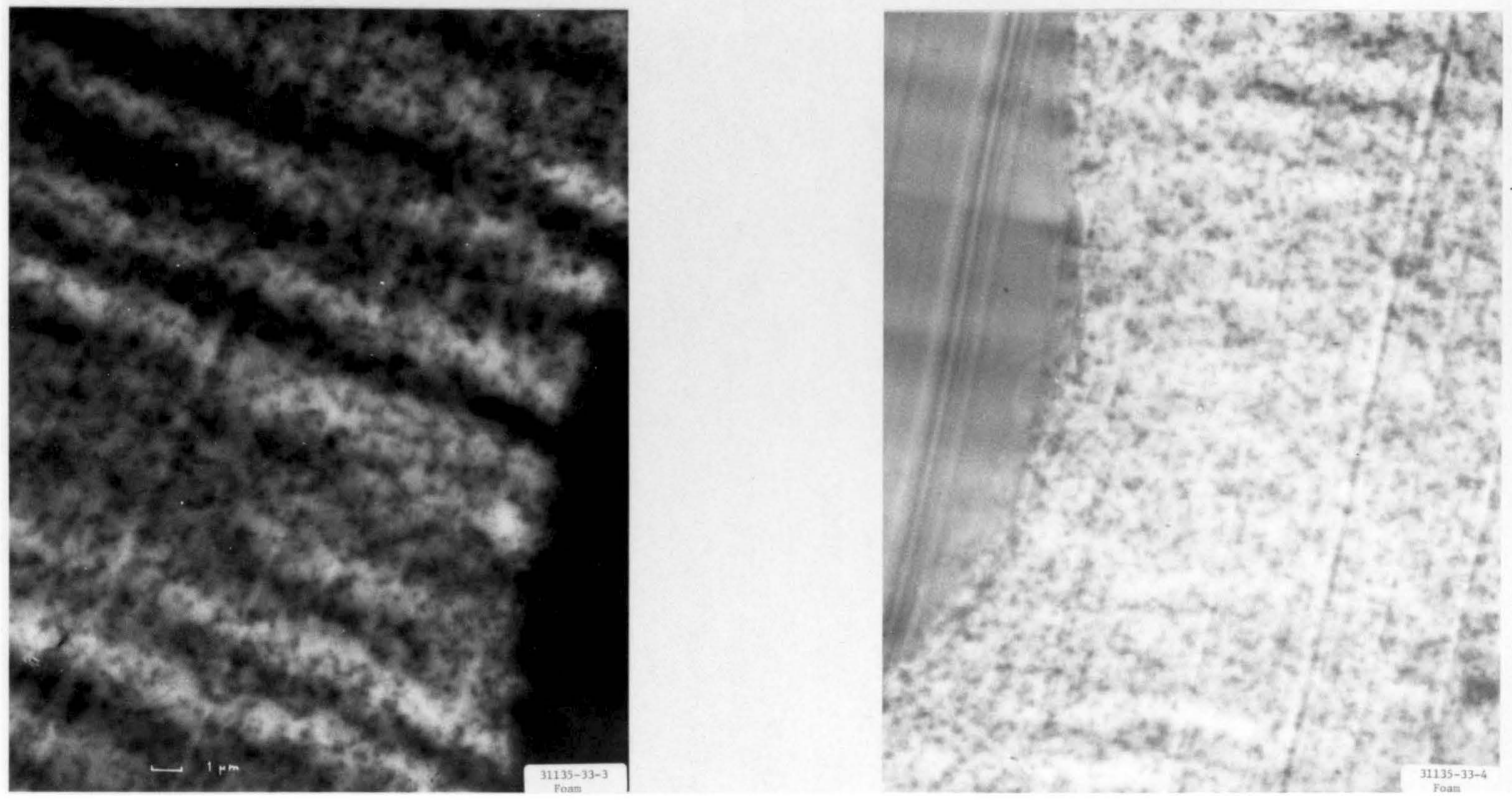

Figure 4.17: TEM micrographs for foams 1 through 4 . 

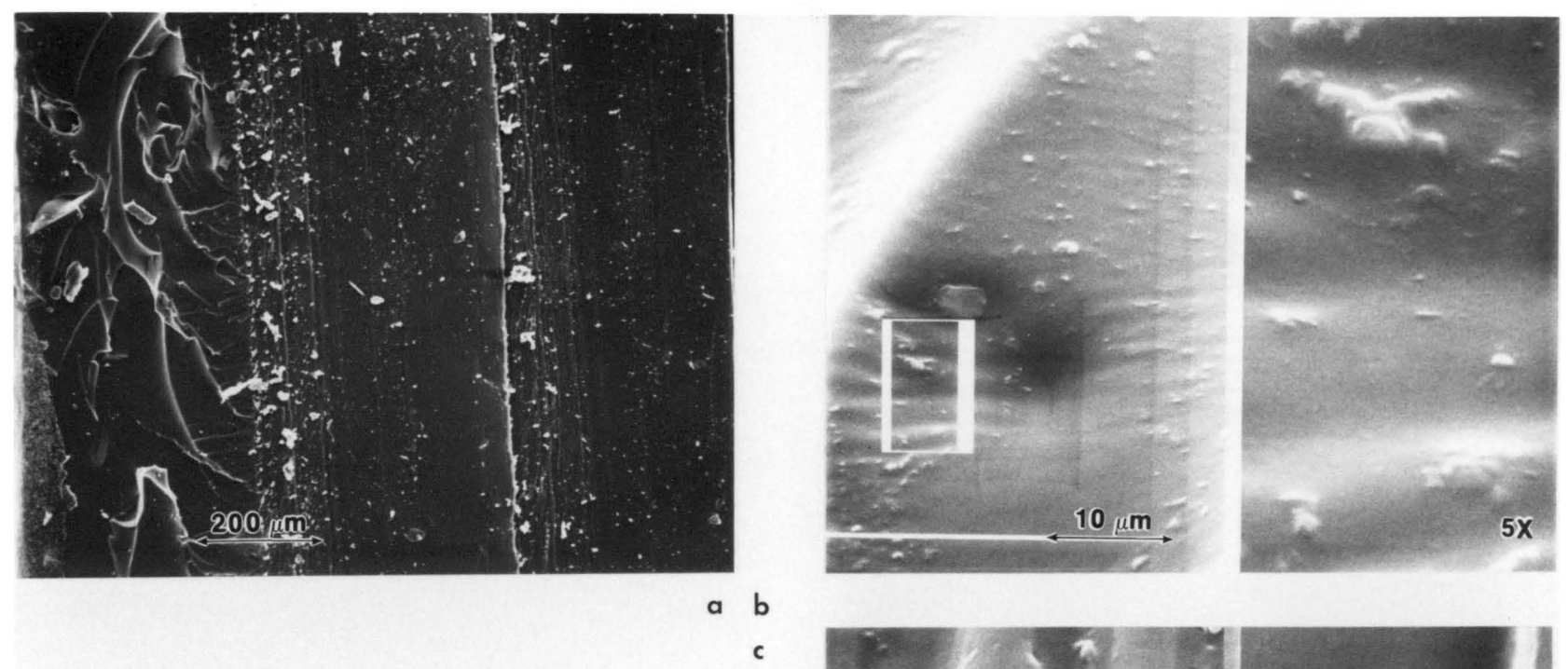

Series 3113533 Plaque 1

(2 pph water, roughly $25 \mathrm{wt}$. \% hard segment)

a) cross-section of fracture surface showing change in texture across the thickness of the plaque due to fracture mechanism

b,c) split-screen close-ups showing texture of surface, right half is $5 \mathrm{X}$ of boxed area

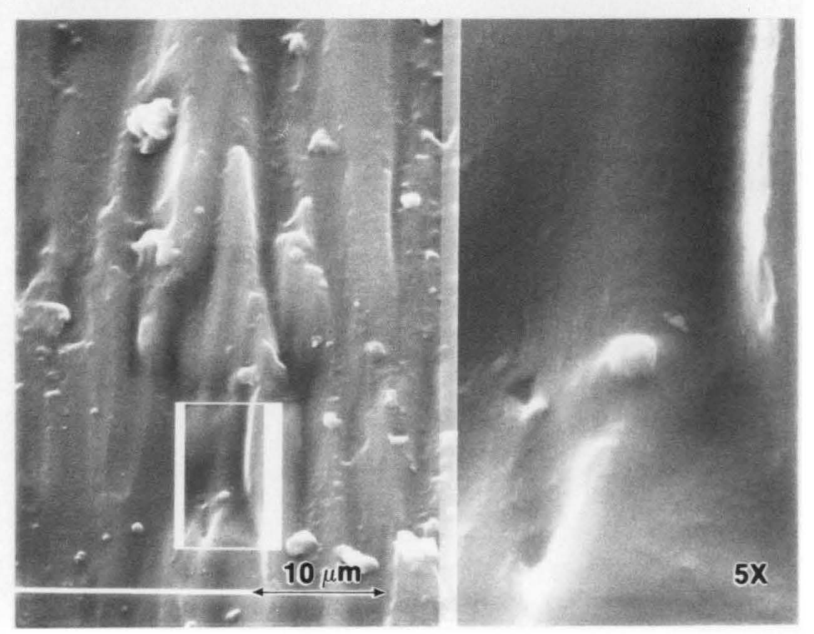

Figure 4.18: SEM micrographs of cold fracture surfaces for plaque 1. 

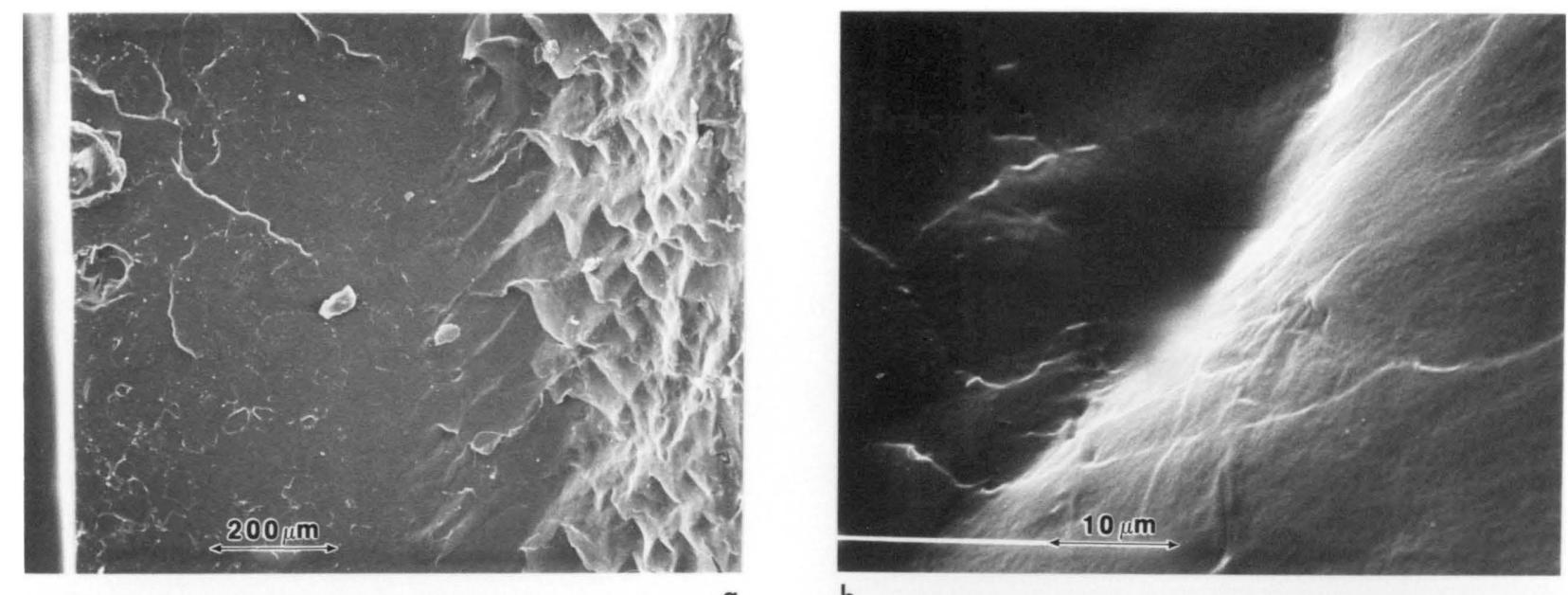

Series 3113533 Placue 2

(3 pph water, roughly $30 \mathrm{wt}$. \% hard segment)

a) cross-section of fracture surface showing change in texture across the thickness of the plaque due to fracture mechanism

b) close-up showing texture of surface

c) split-screen picture emphasizing texture brought out by b), right half of screen is $5 \mathrm{X}$ of boxed are

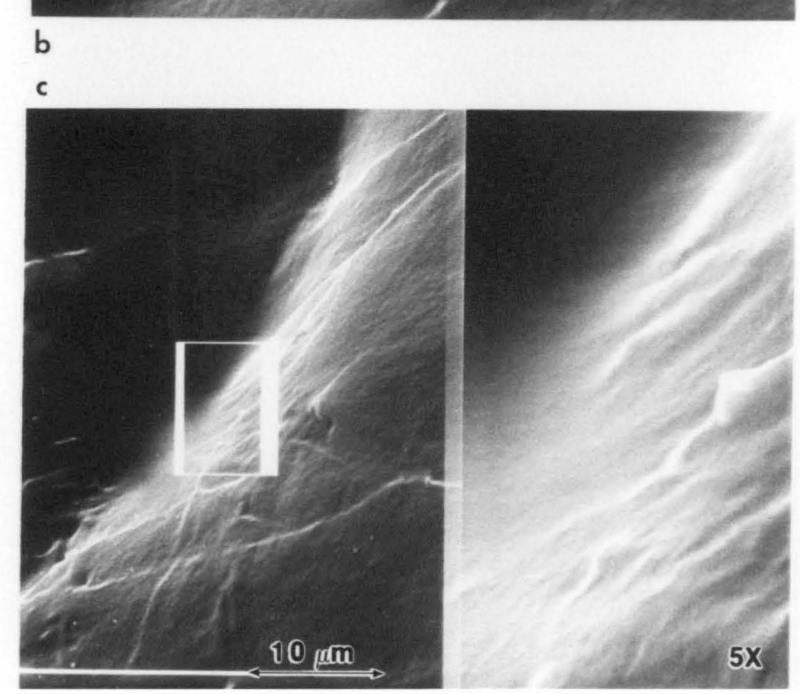

Figure 4.19: SEM micrographs of cold fracture surfaces for plaque 2. 

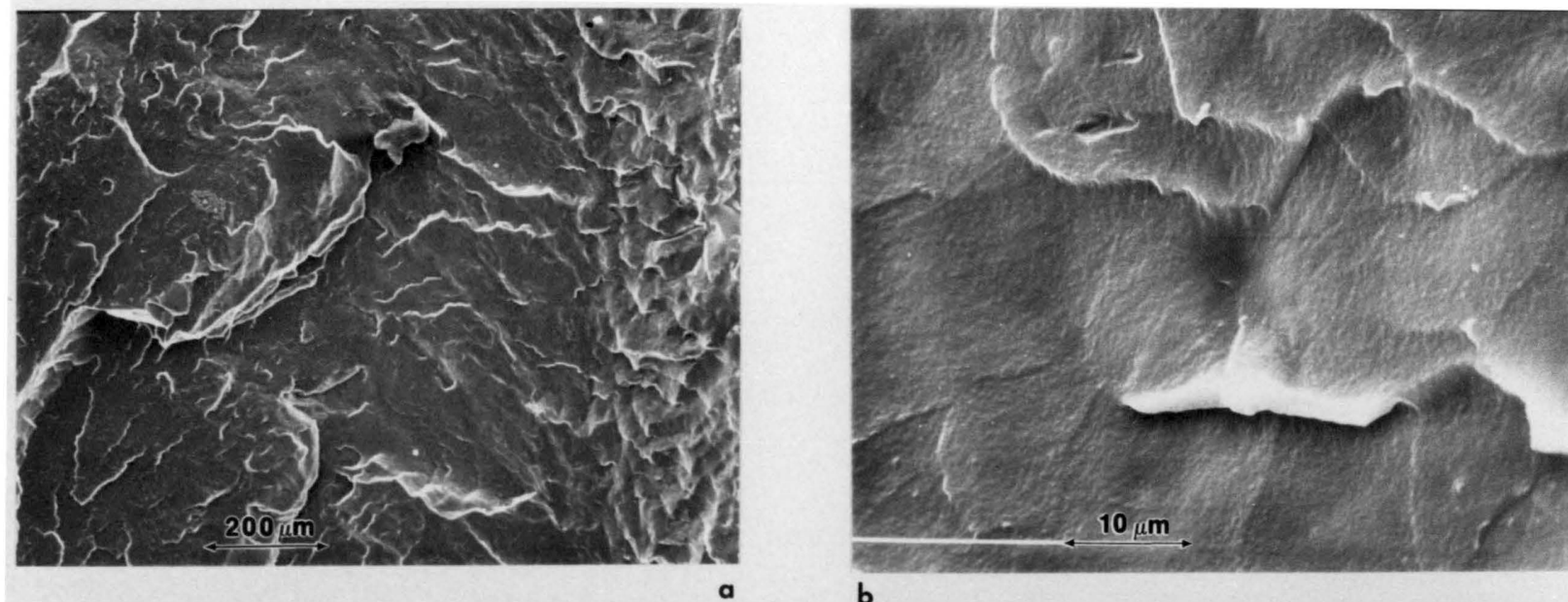

a

Series 3113533 Plaque 3

(4 pph water, roughly $35 \mathrm{wt}$. \& hard segment)

a) cross-section of fracture surface showing change in texture across the thickness of the plaque due to fracture mechanism

b) close-up showing texture of surface

c) split-screen picture emphasizing texture brought out by b), right half of screen is $5 \mathrm{X}$ of boxed area

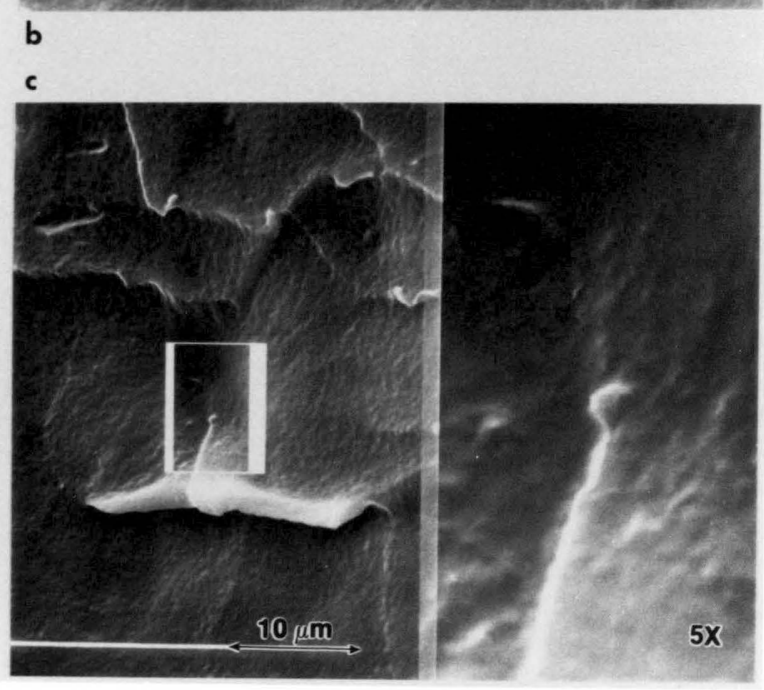

Figure 4.20: SEM micrographs of cold fracture surfaces for plaque 3. 

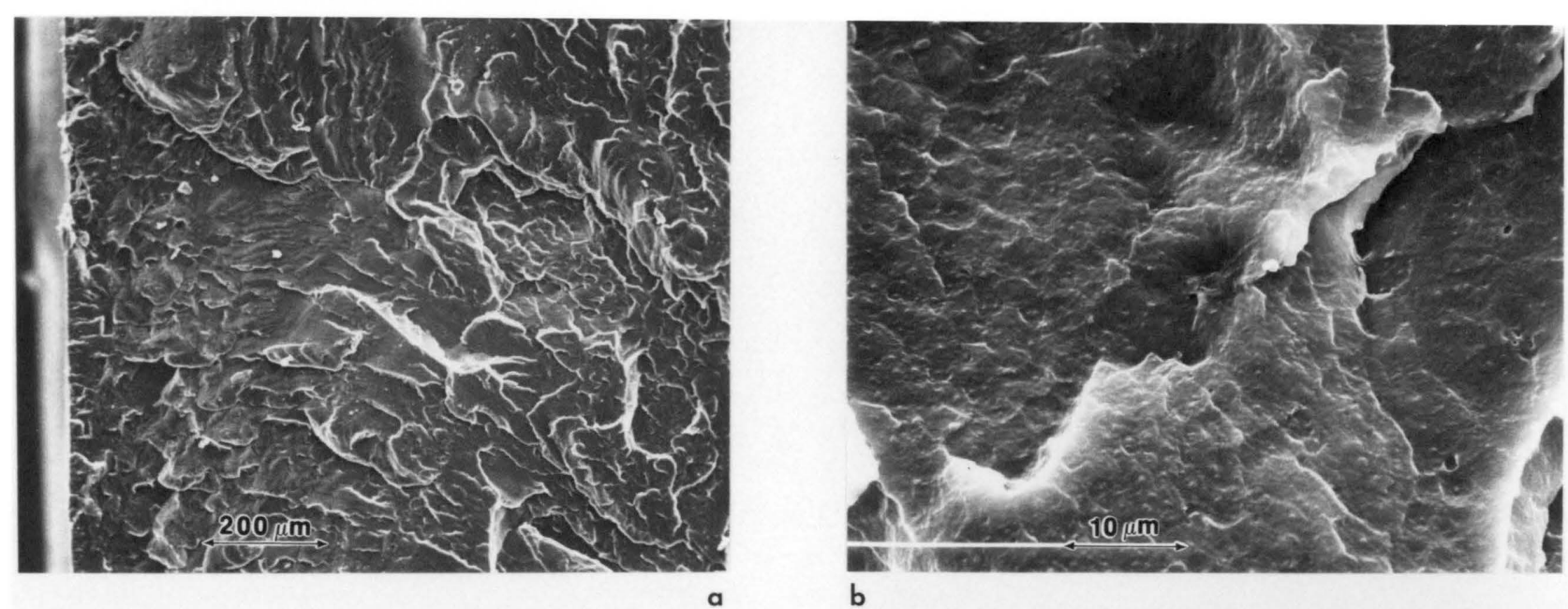

Series 3113533 Plaque 4

(5 pph water, roughly 40 wt. \% hard segment)

a) cross-section of fracture surface showing change in texture across the thickness of the plaque due to fracture mechanism

b) close-up showing texture of surface

c) split-screen picture emphasizing texture brought out by b), right half of screen is $5 \mathrm{X}$ of boxed area

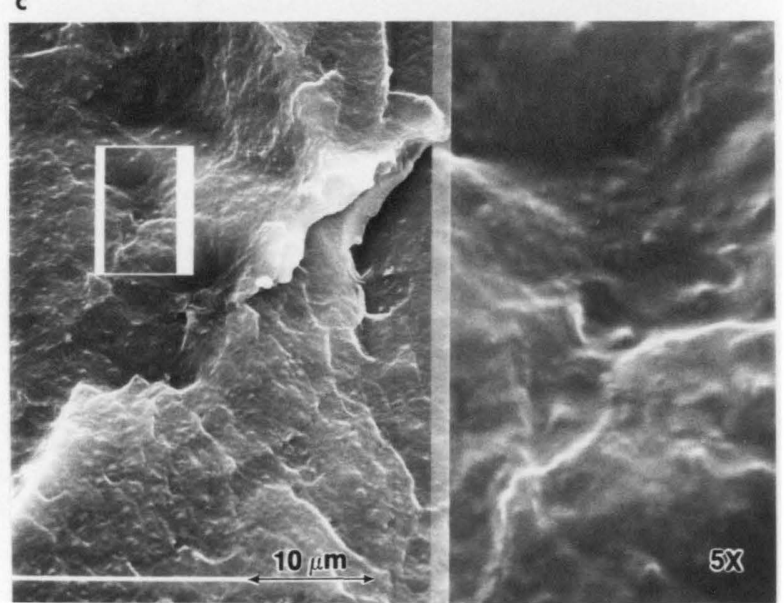

Figure 4.21: SEM micrographs of cold fracture surfaces for plaque 4 . 

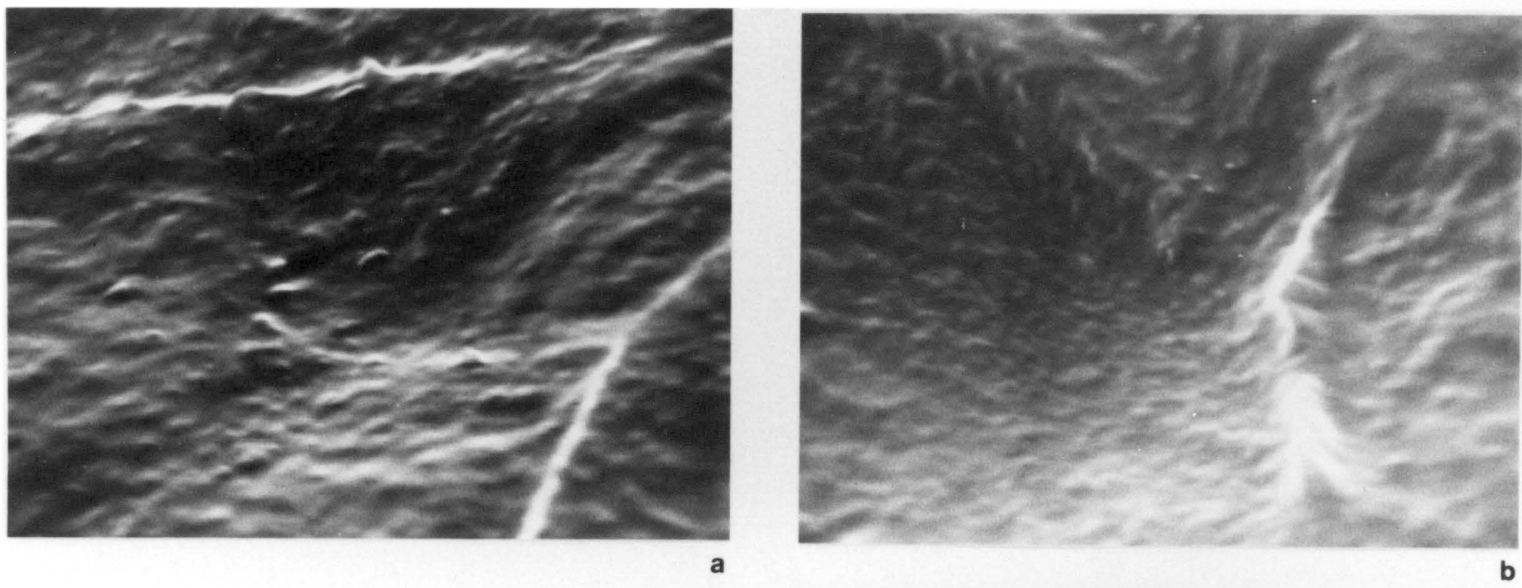

Ion Milling of Cold Fracture Surfaces Plaque 31135-33-5

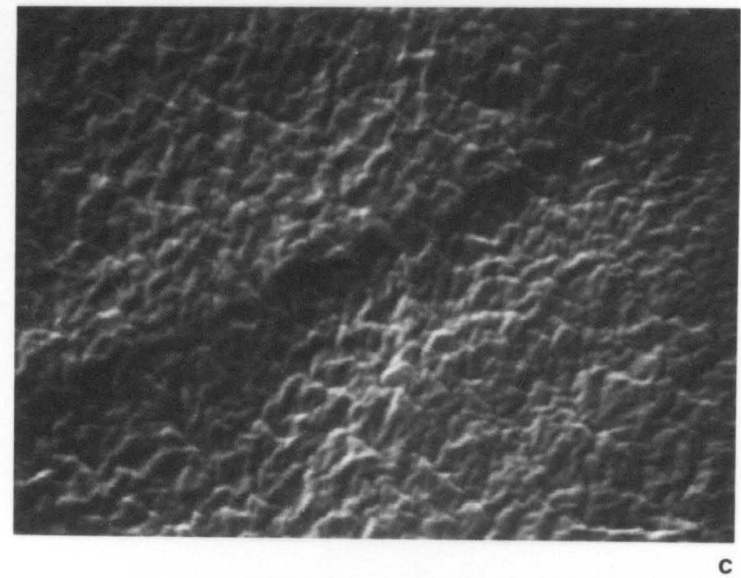

b) 5 minutes

c) 12 minutes

d) 16 minutes

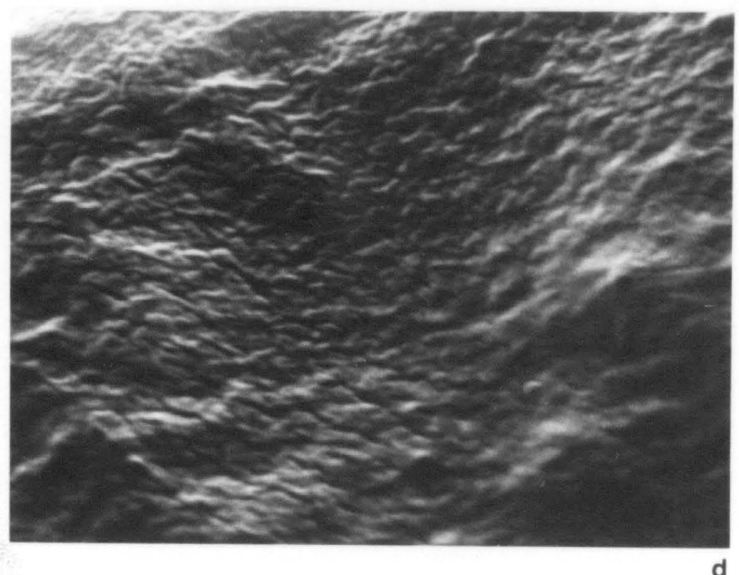

Figure 4.22: SEM micrographs of ion milled cold fracture surfaces for plaque 5. 

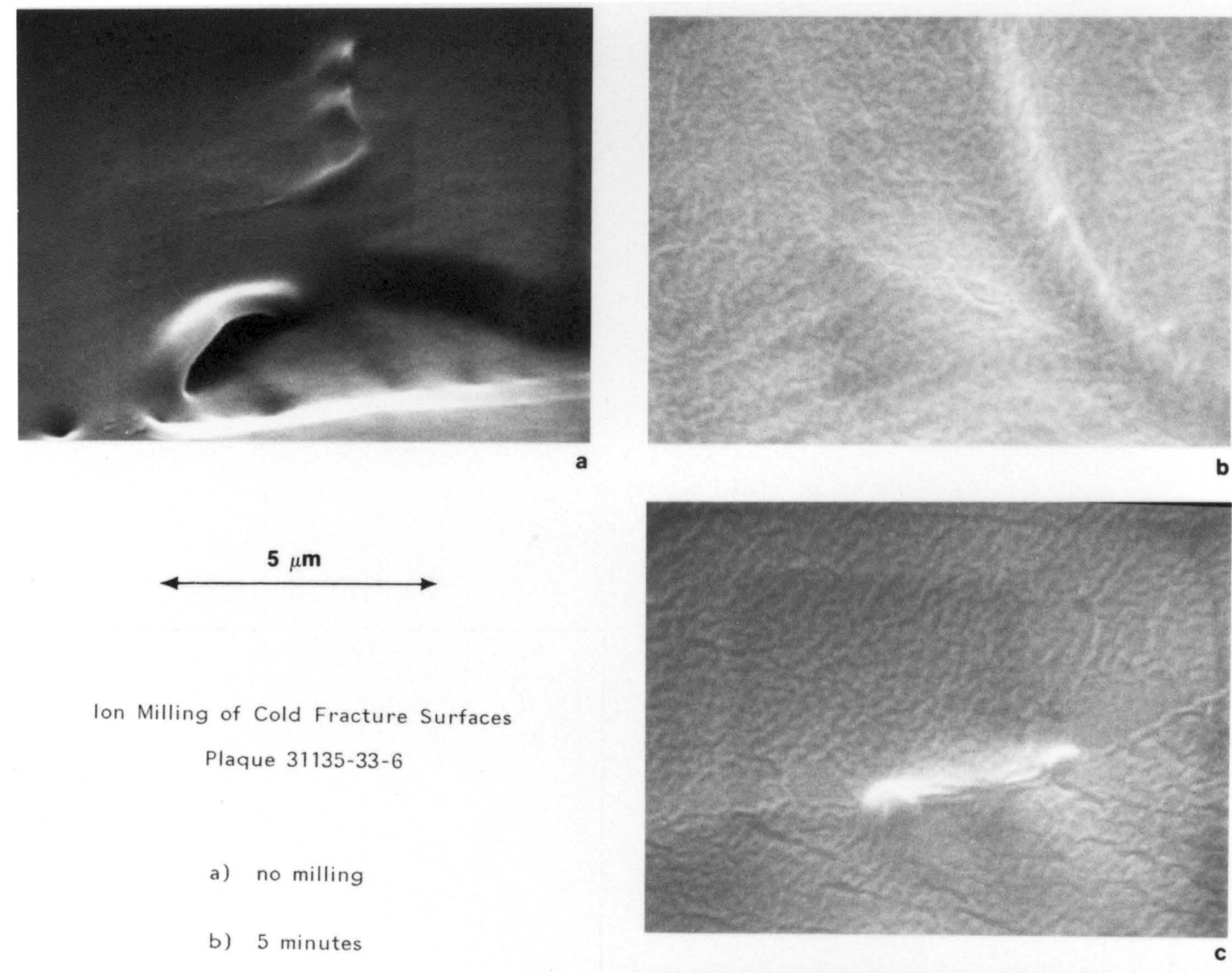

b) 5 minutes

c) 12 minutes

d) 17.5 minutes

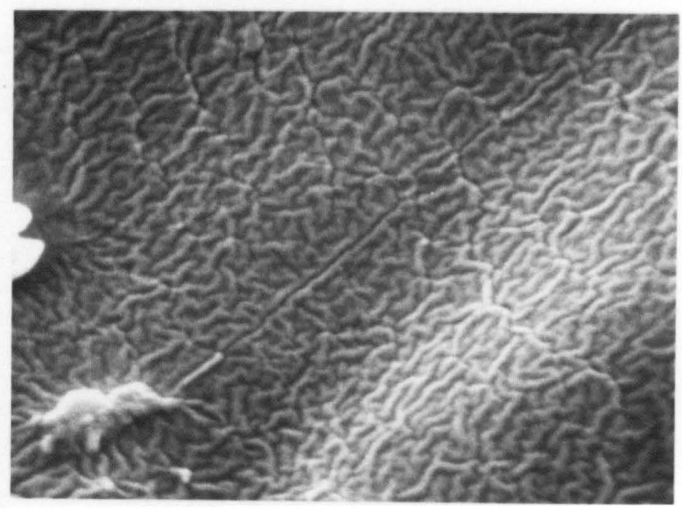

Figure 4.23: SEM micrographs of ion milled cold fracture surfaces for plaque 6. 


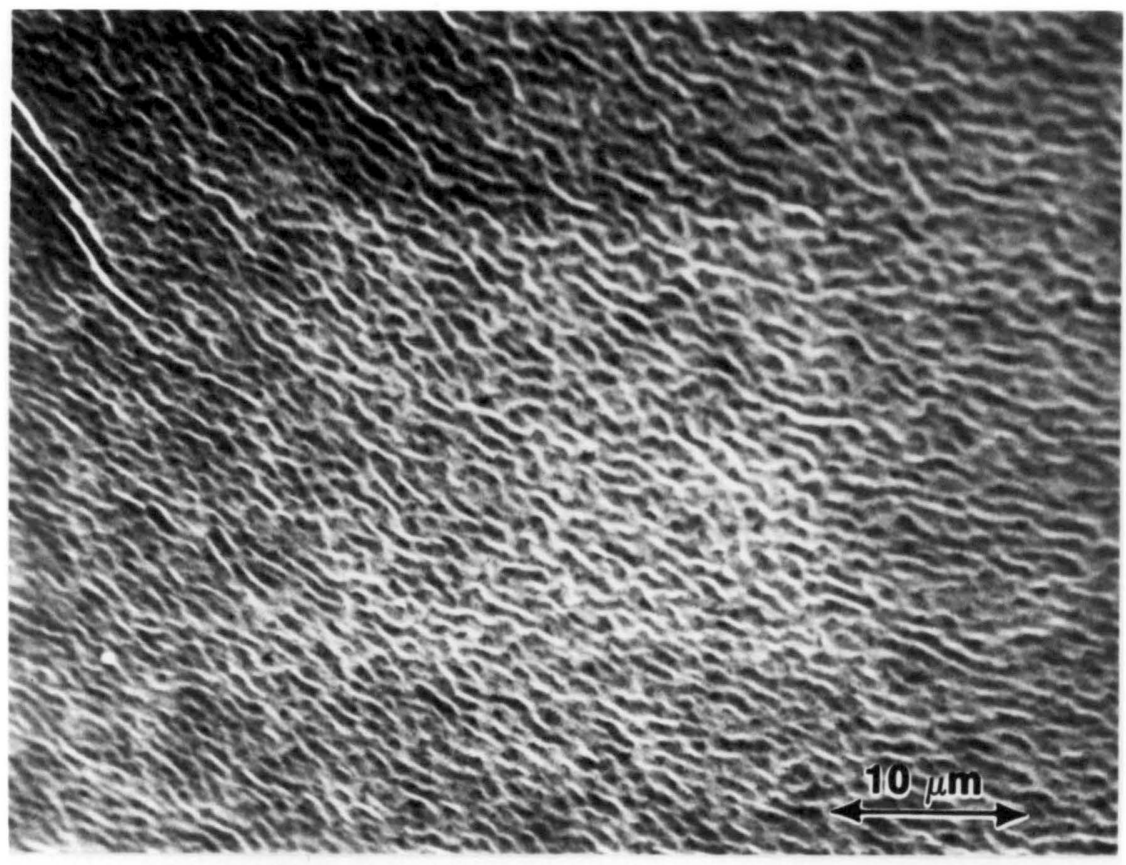

a

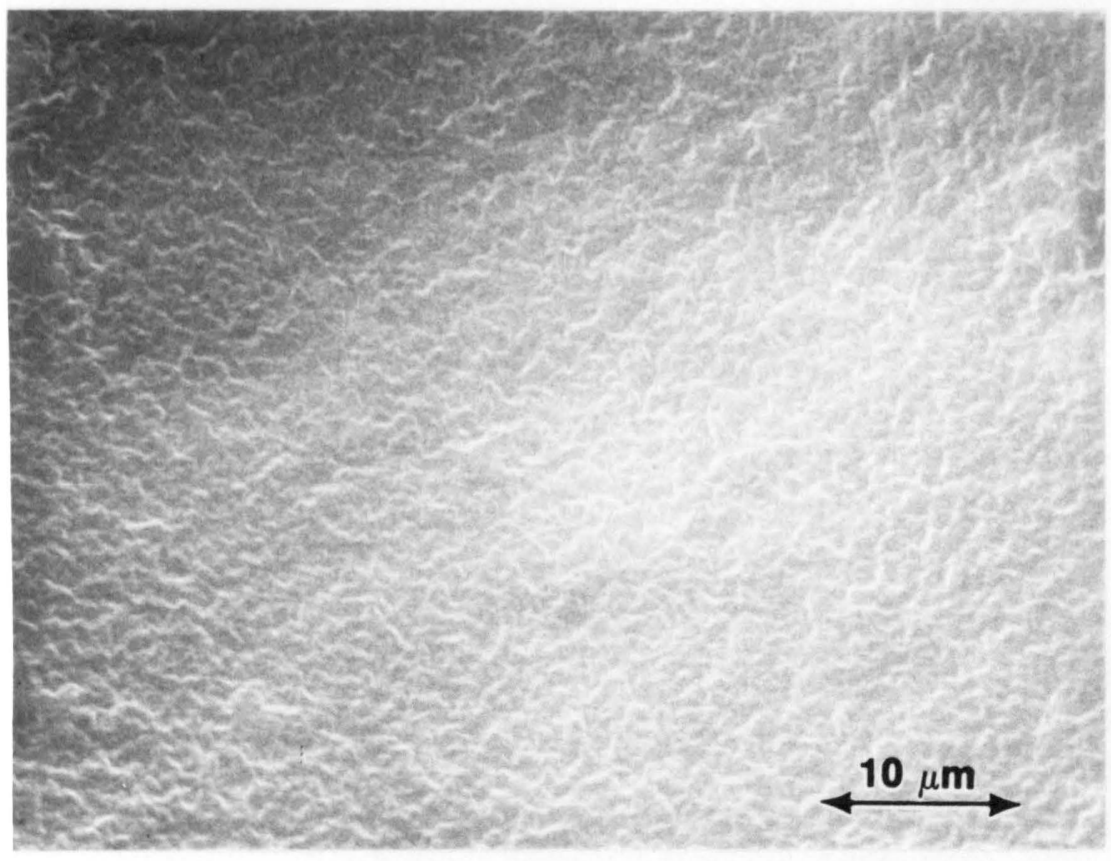

b

Figure 4.24: SEM micrographs of the surfaces of foam struts for a) foam 1 and b) foam 4 . 

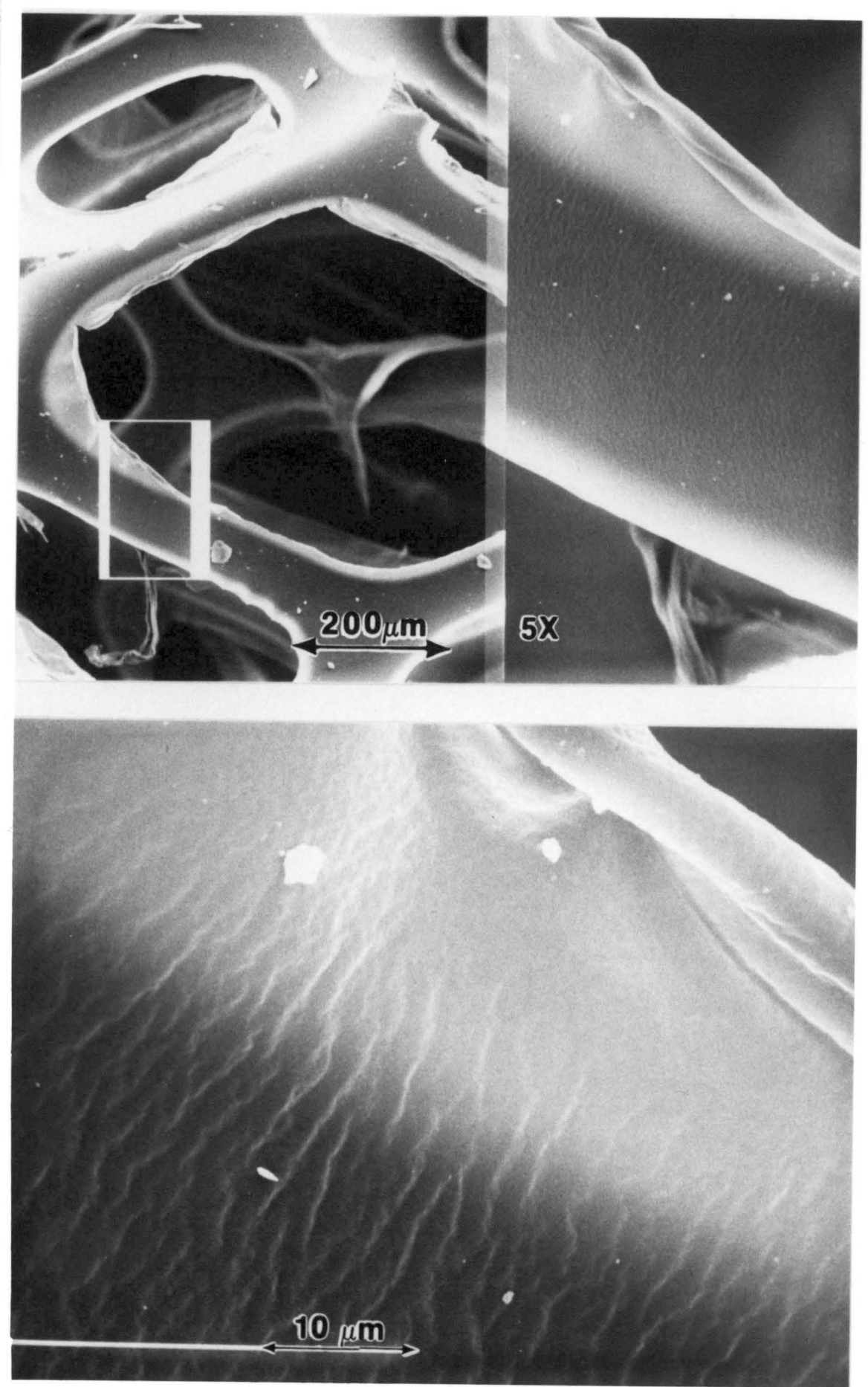

Figure 4.25: Lower magnification micrographs of strut surfaces revealing a texture transverse to the strut axis (foam $31857-45-5)$. 


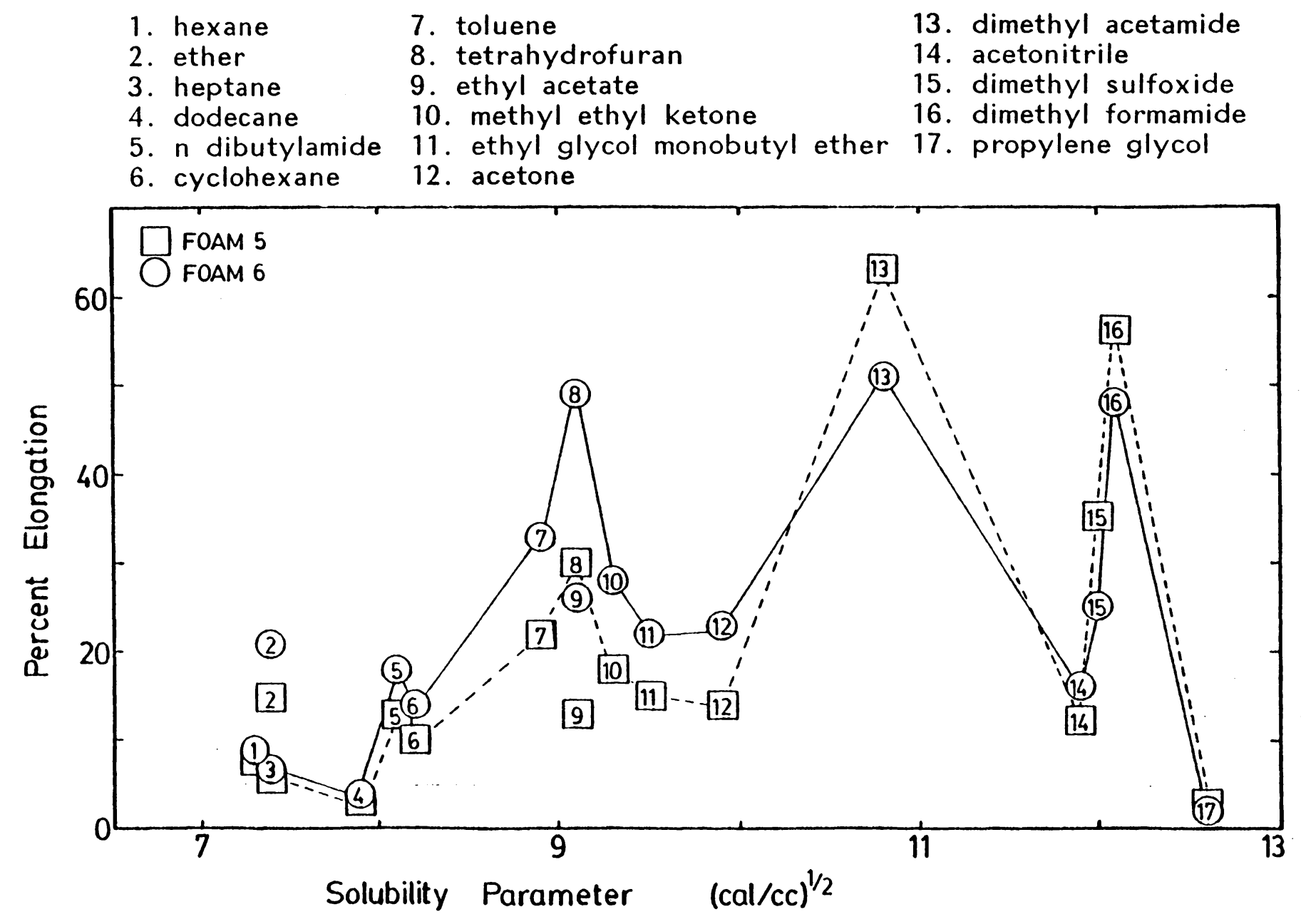

$\stackrel{\triangleright}{\triangleright}$

Figure 4.26: Swelling of foams 5 and 6 in various solvents. 


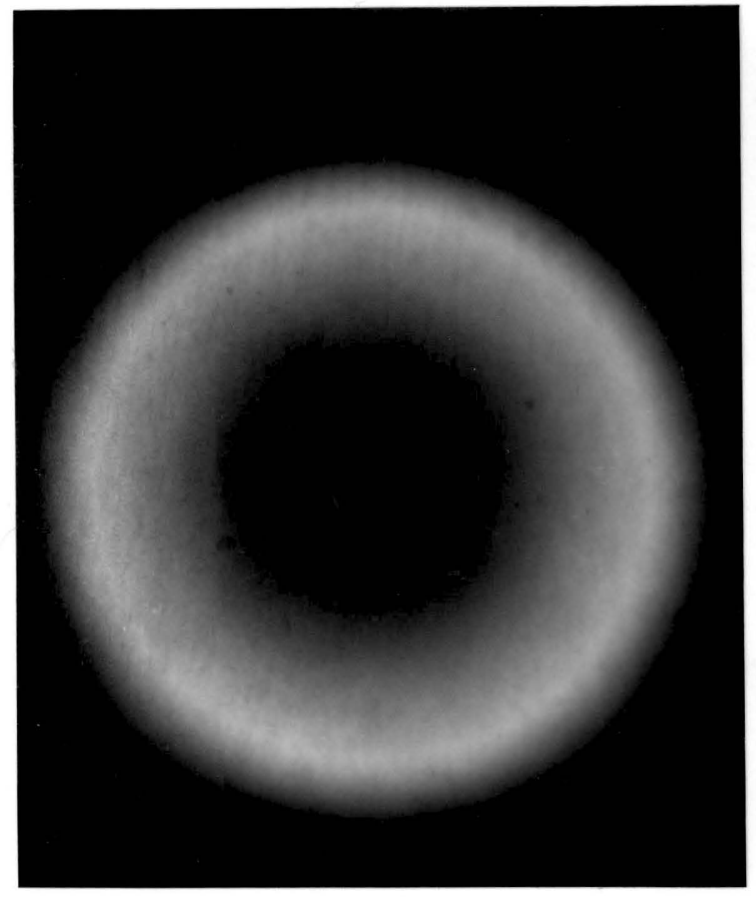

FOAM 1

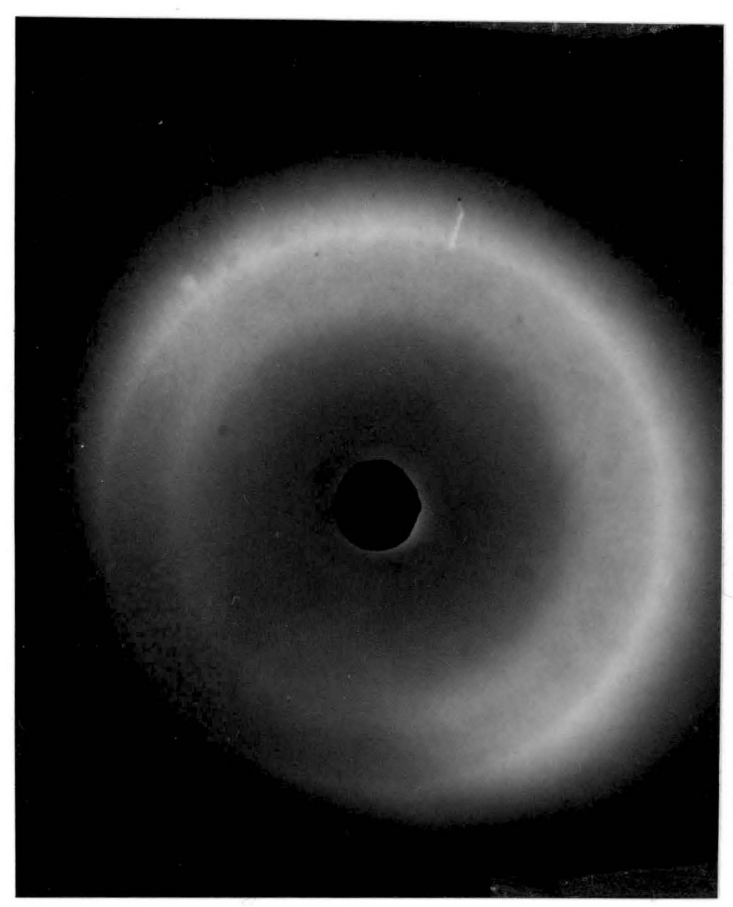

FOAM 3

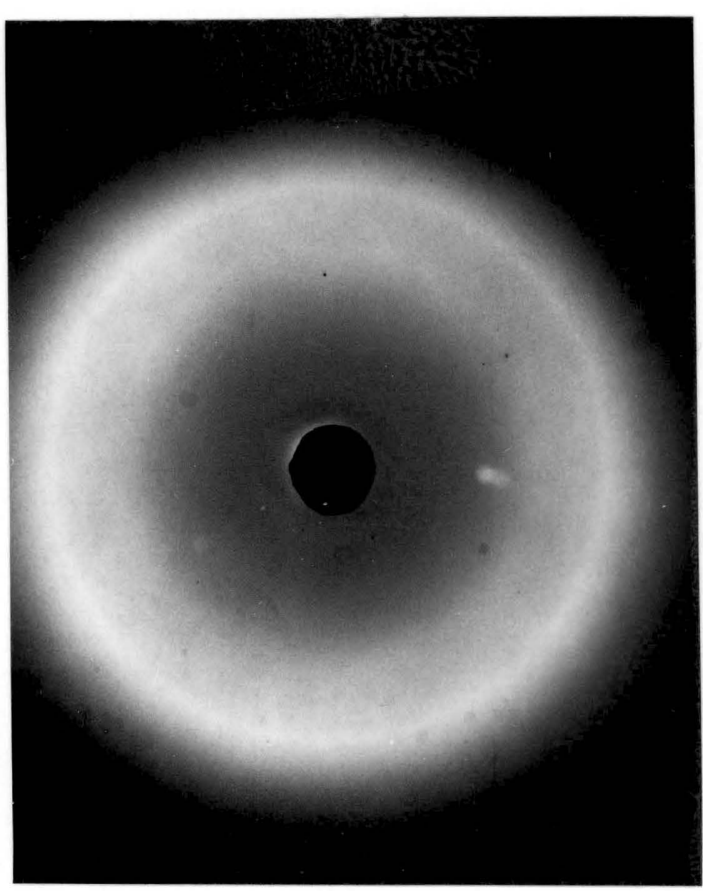

FOAM 2

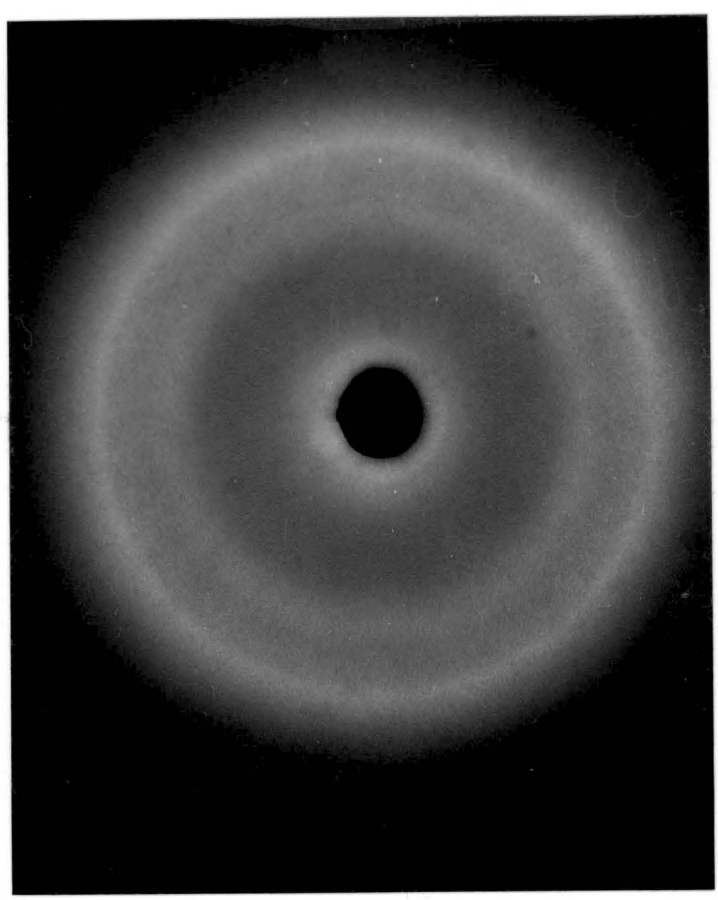

FOAM 4

Figure 4.27: WAXS patterns for foams 1 through 4 . 


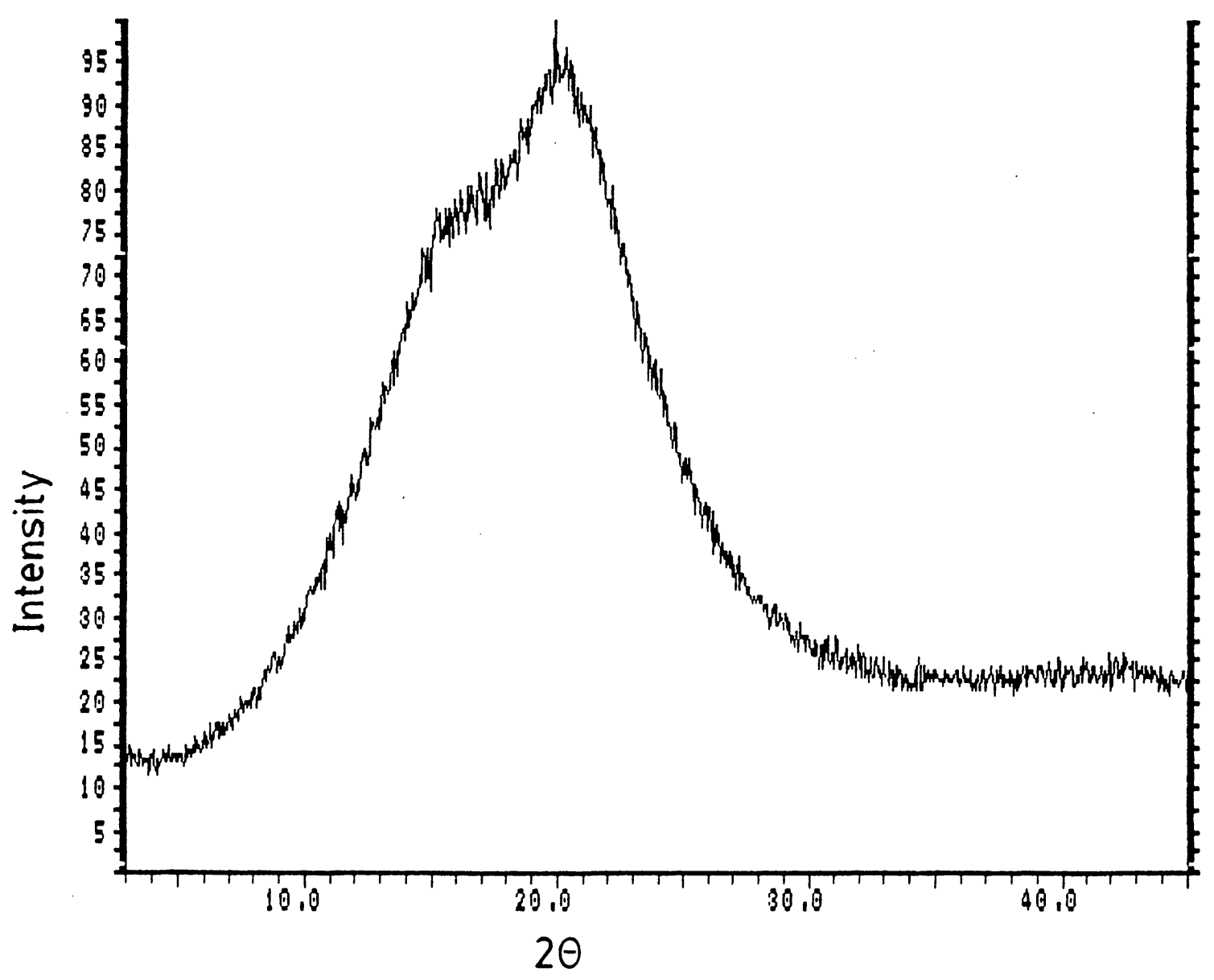

Figure 4.28: Diffractometer scan of plaque 4. 


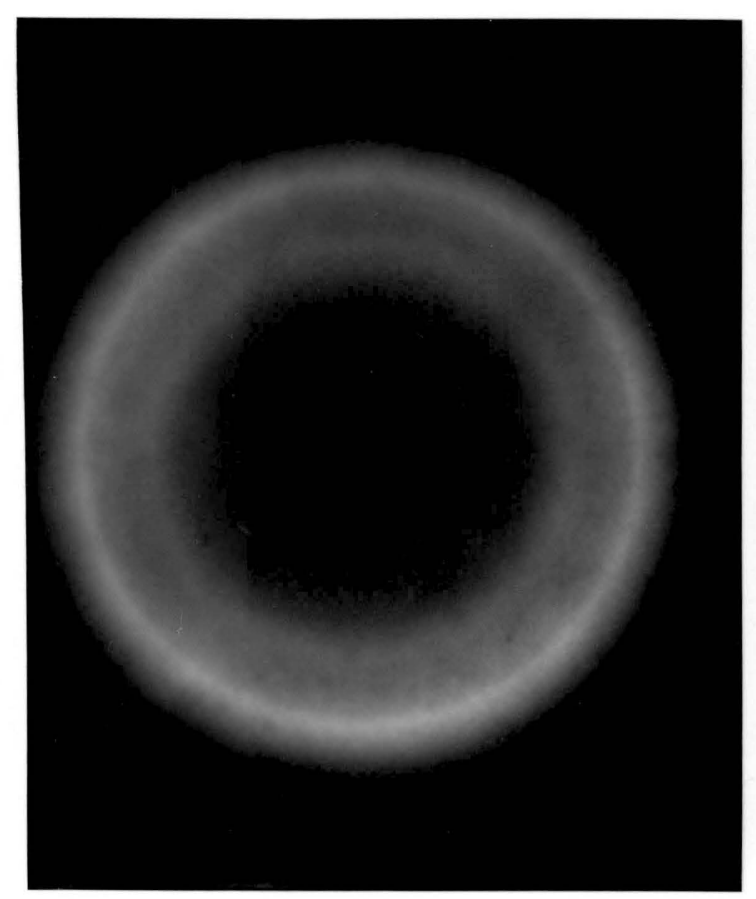

before

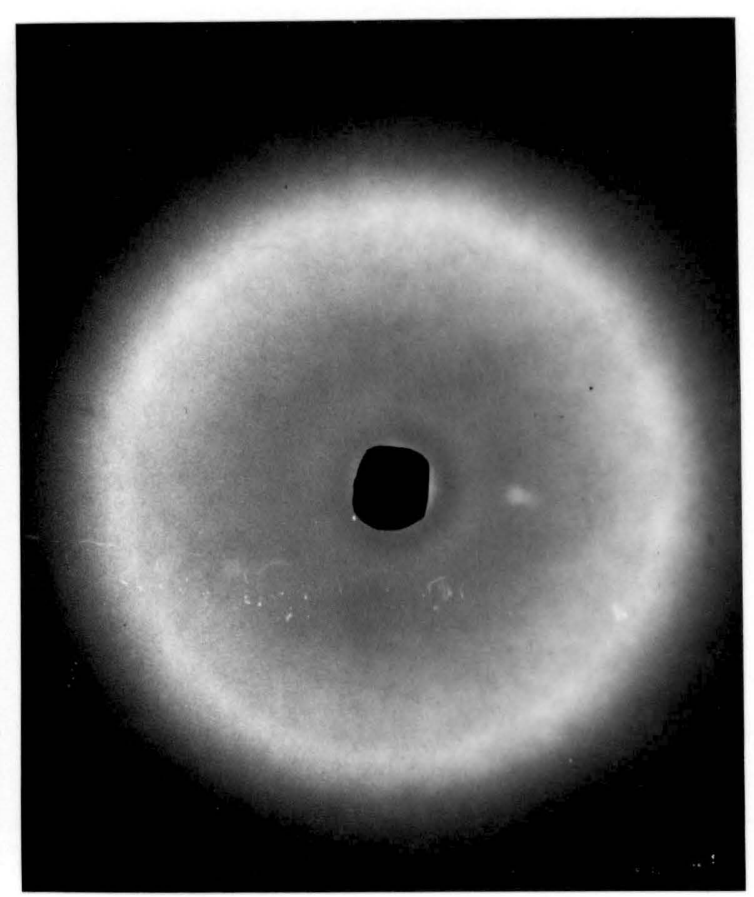

after

Figure 4.29: WAXS patterns for plaque 4 before and after extraction with DMF. 

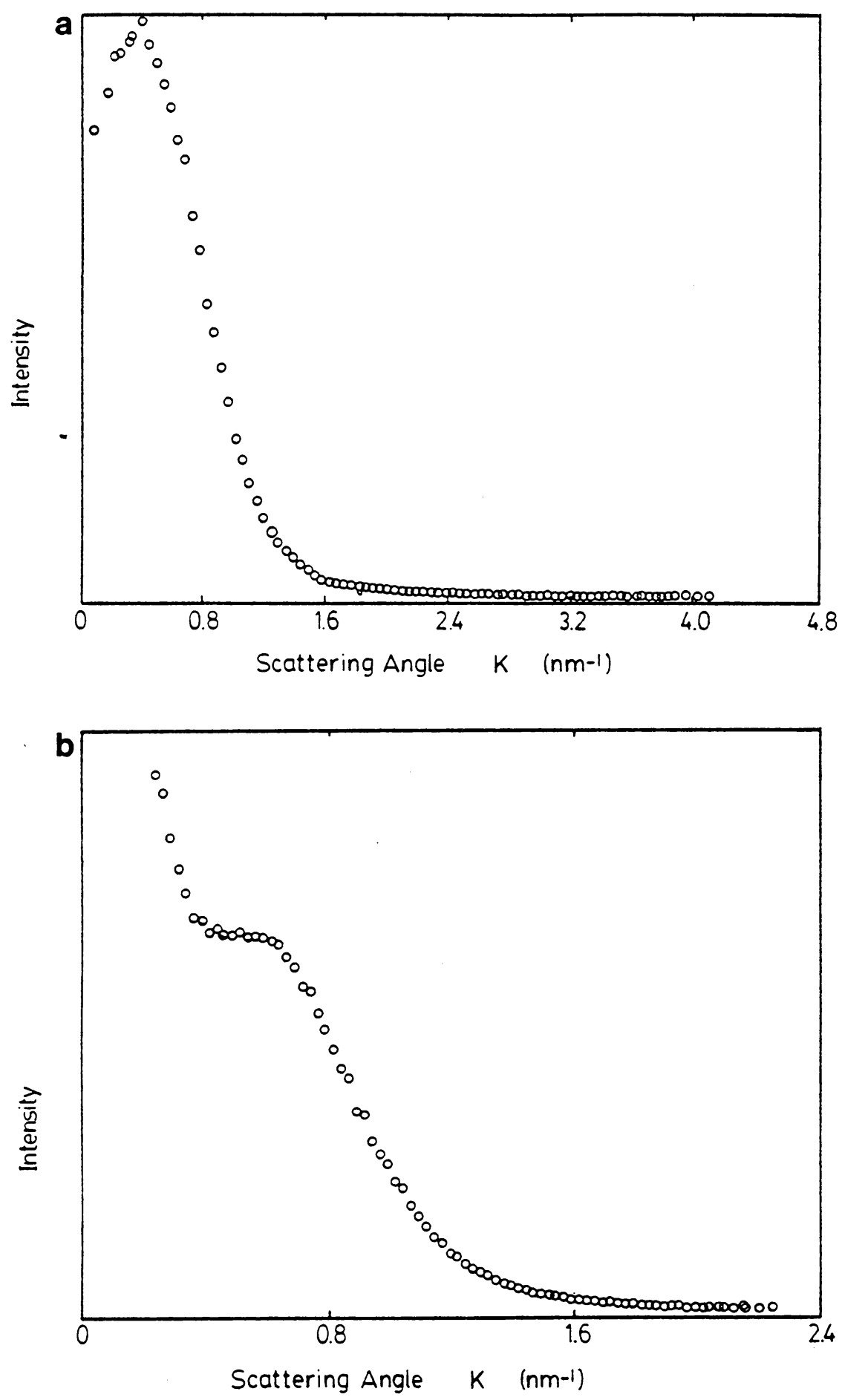

Figure 4.30: Radial average scattering profiles for plaque 4. Data collected on ORNL 10-meter SAXS instrument at a) SDD $=1$ meter and b) $S D D=2$ meters. 

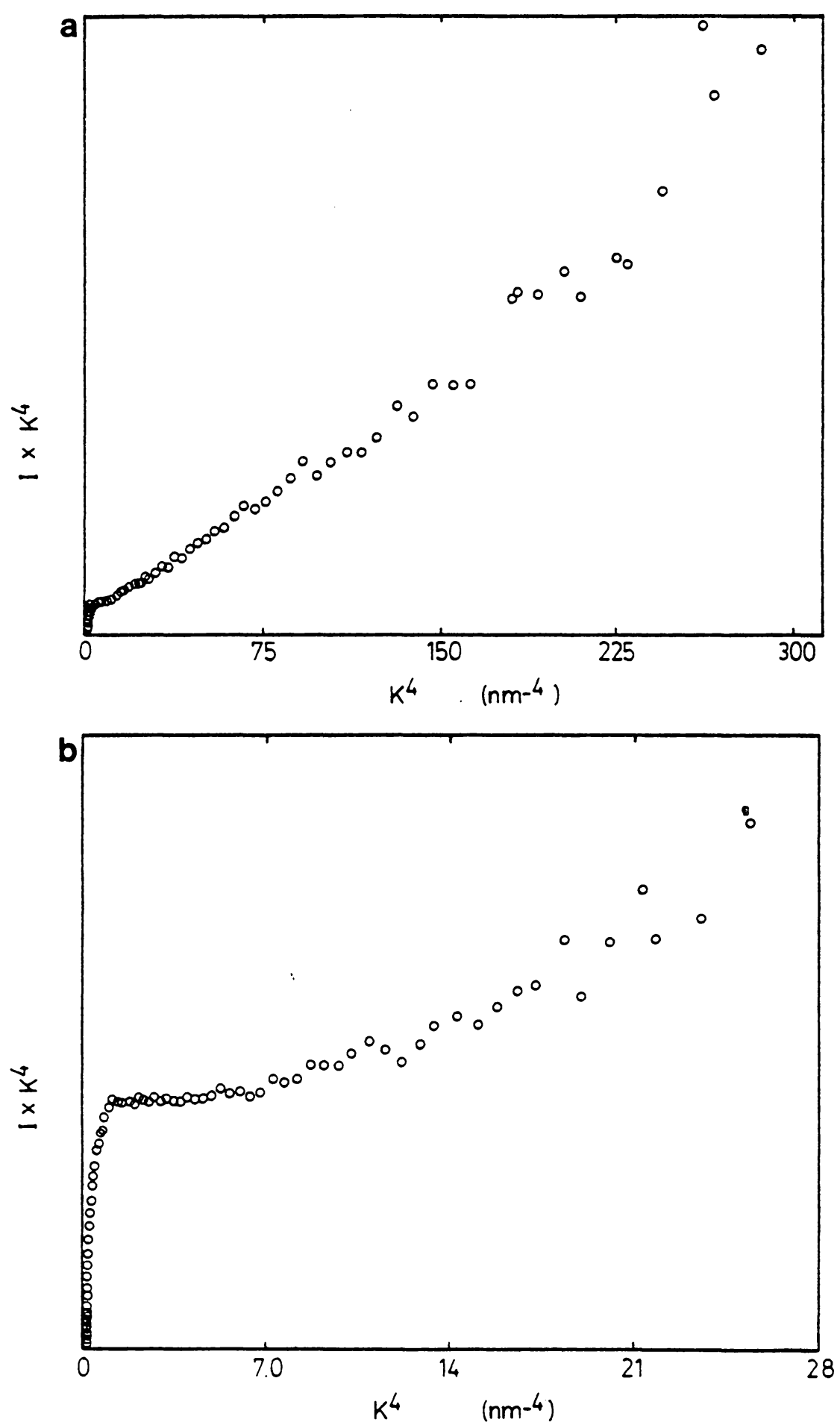

Figure 4.31: Bonart plots for the estimation of background scattering. Note the scale difference for plot a) 1-meter data and plot b) 2-meter data. 


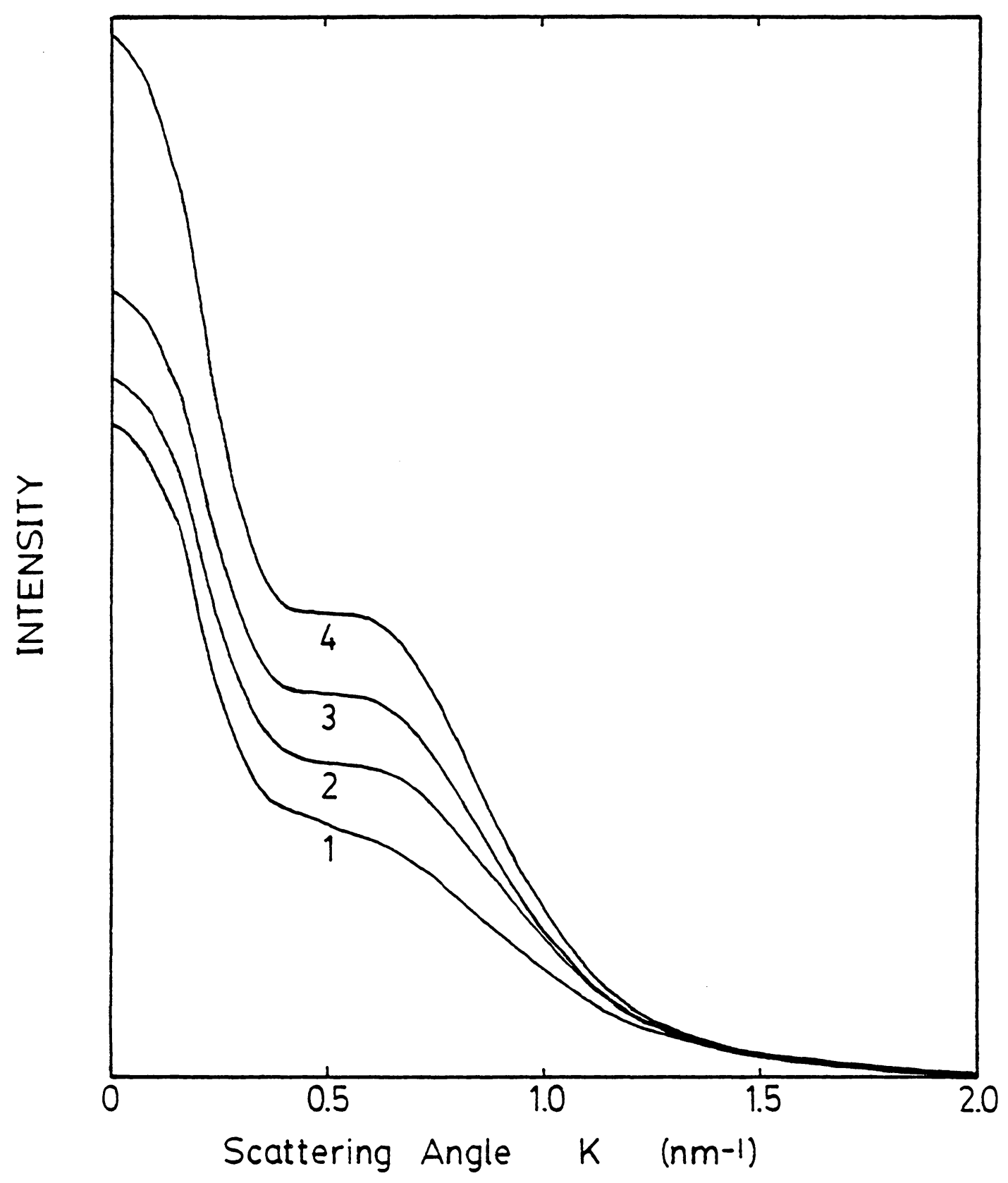

Figure 4.32: Scattering profiles for plaques 1 through 4. Scattering are the result of extrapolation to zero angle, background removal, tail fitting, and smoothing and show correct relative intensity. 


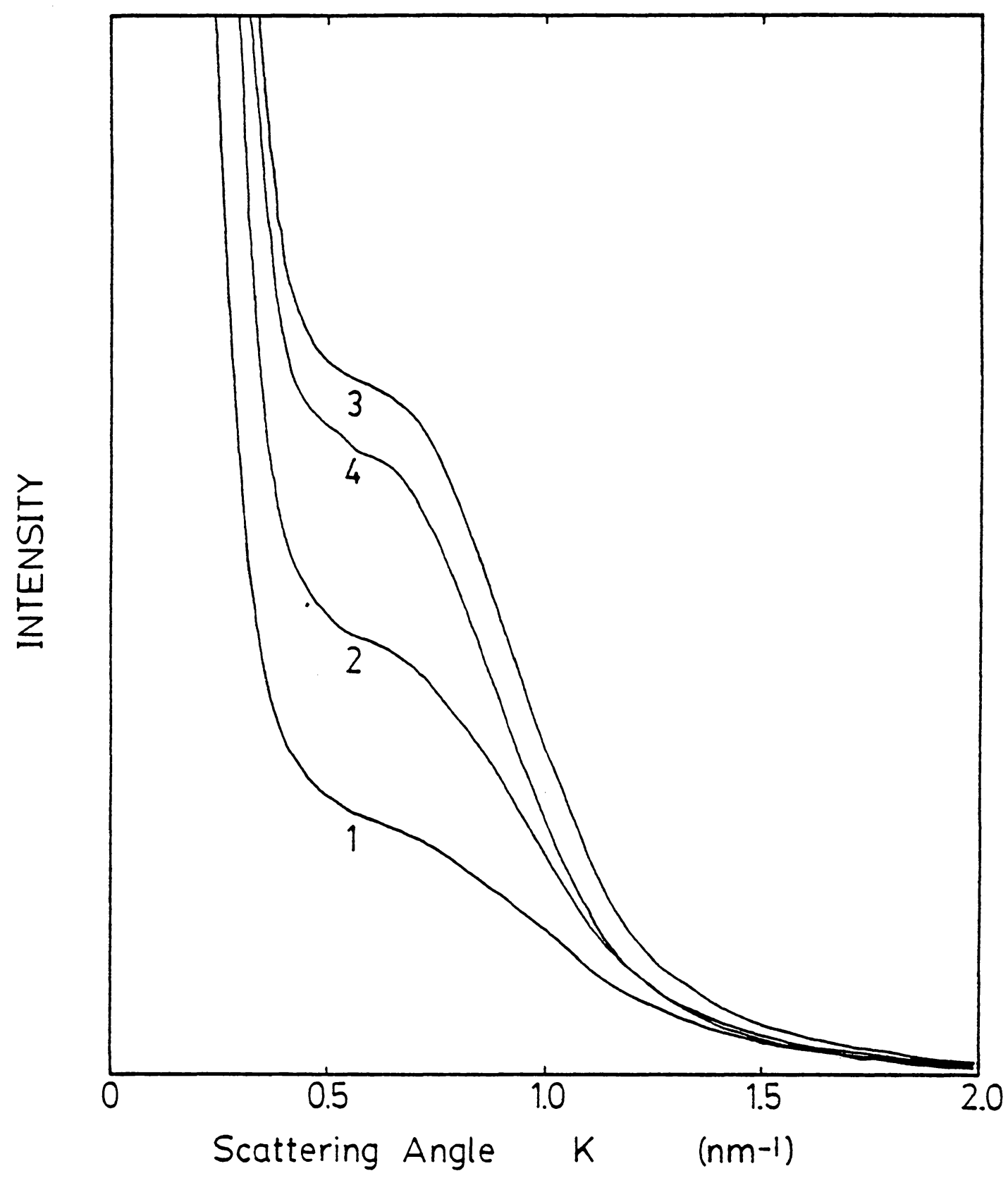

Figure 4.33: Scattering profiles for foams 1 through 4 . Scattering are the result of extrapolation to zero angle, background removal, tail fitting, and smoothing and show correct relative intensity. 


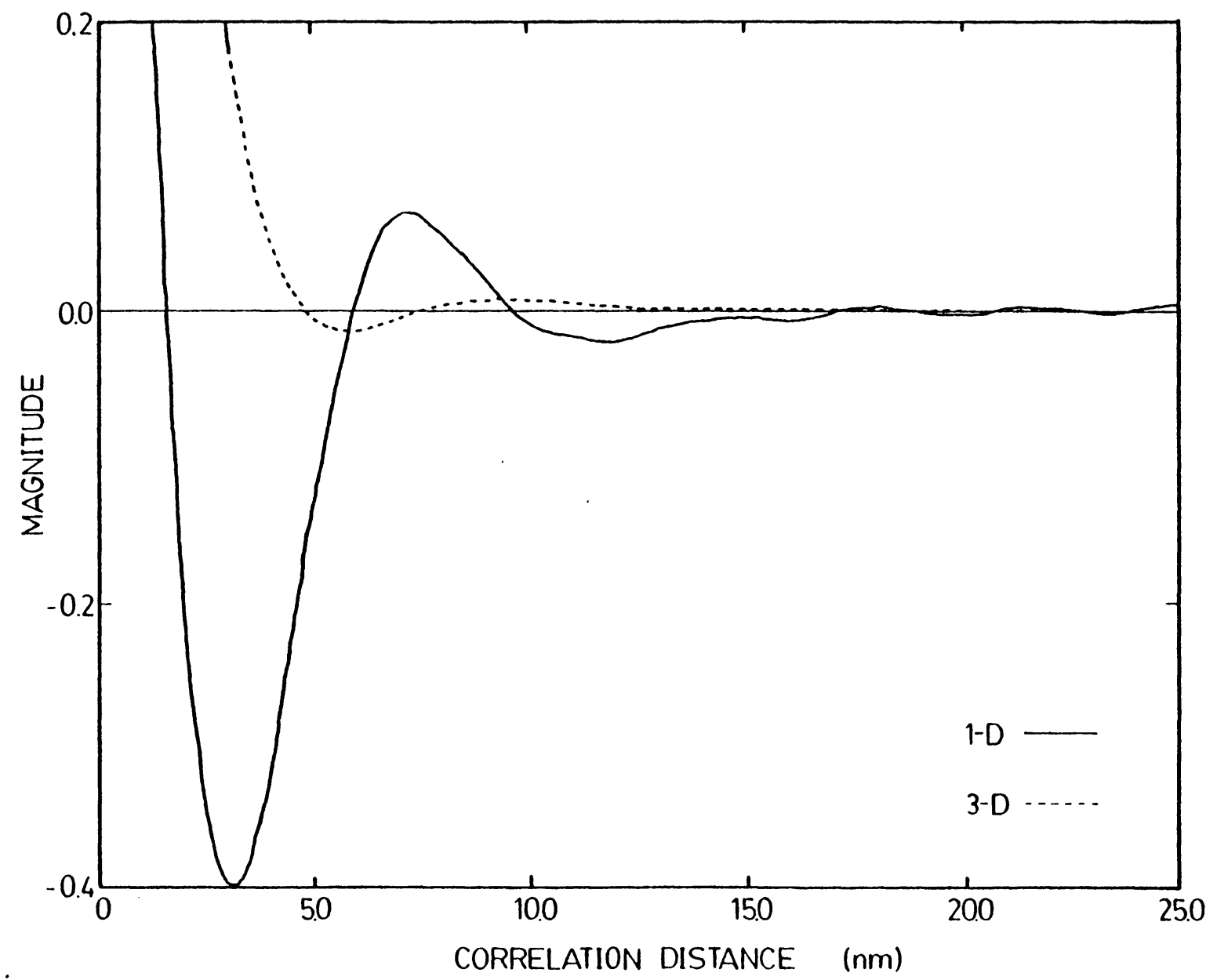

ก

Figure 4.34: Correlation functions for plaque 3. 


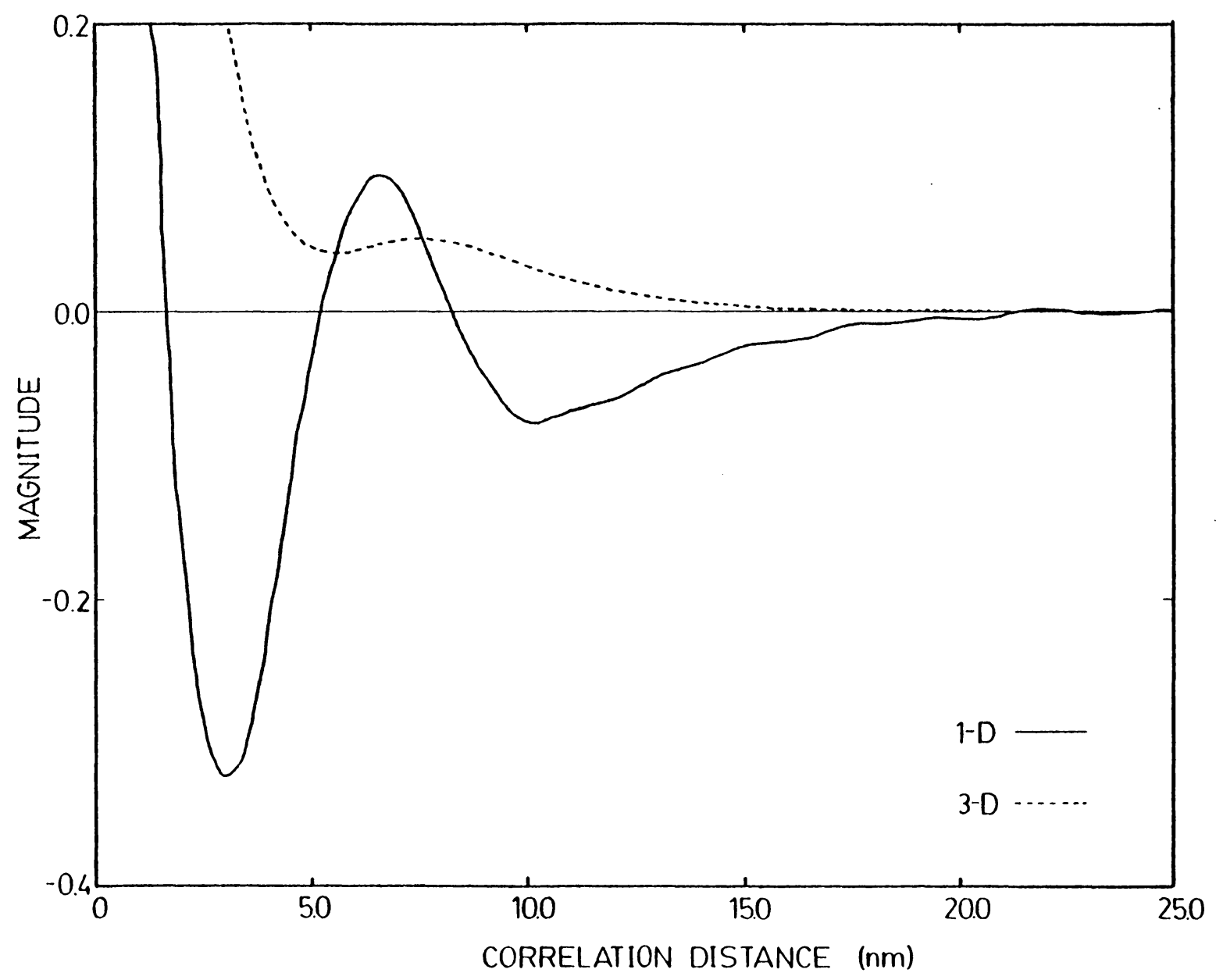

Figure 4.35: Correlation functions for foam 3 . 


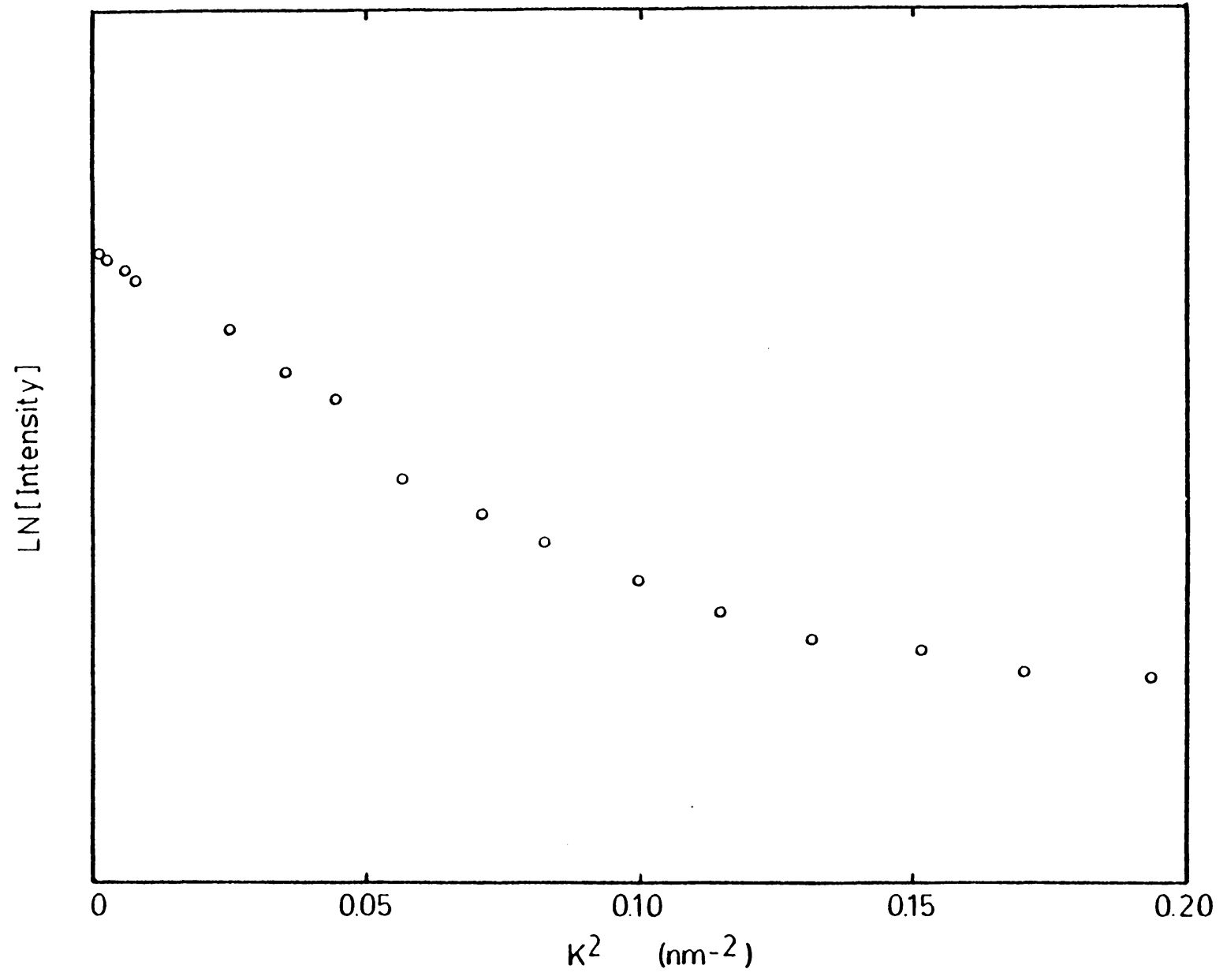

$\widetilde{\circ}$

Figure 4.36: Guinier plot for plaque 2. 


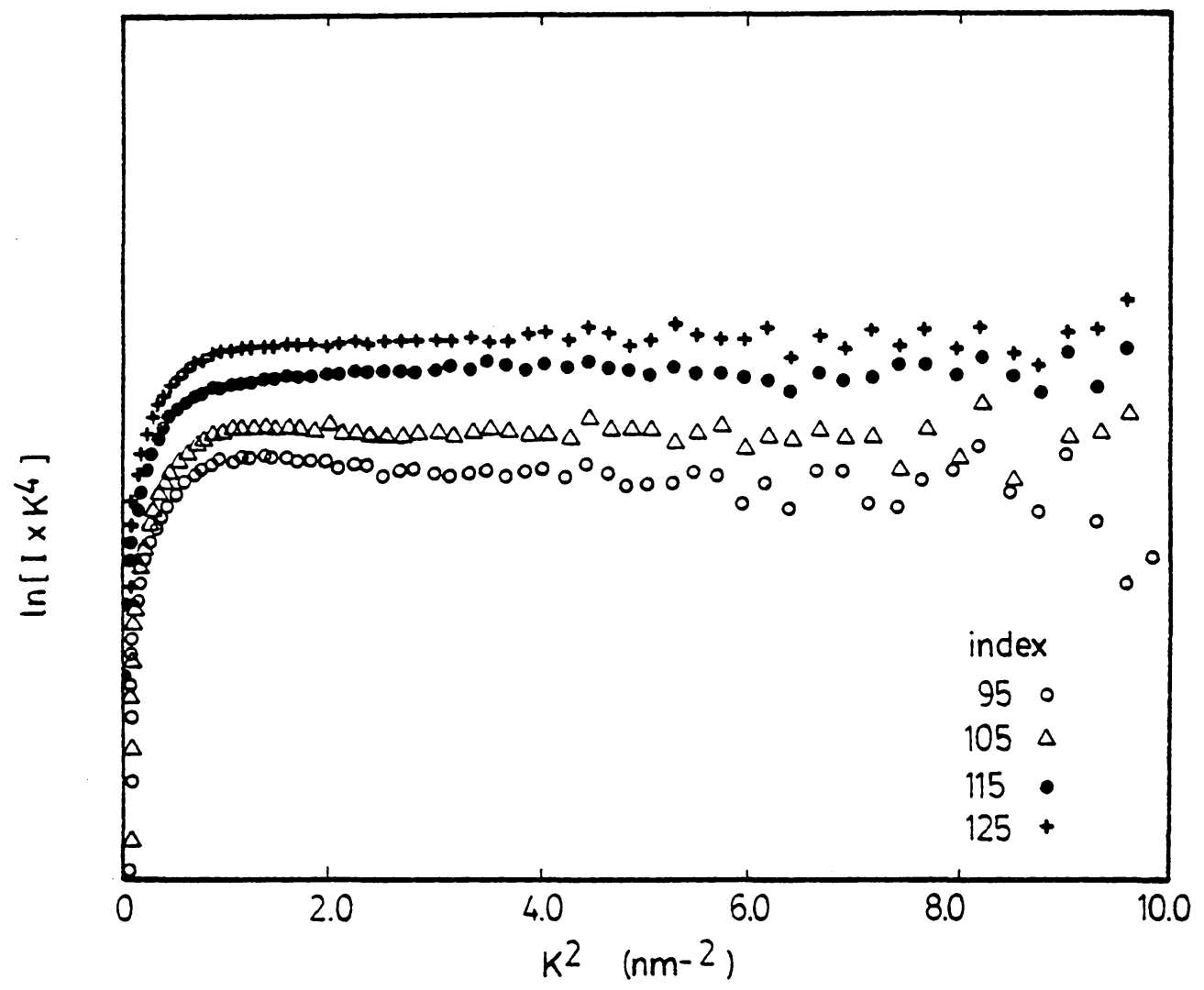

Figure 4.37: Porod's law behavior for 31857-45-X foams showing the influence of increasing isocyanate index. 


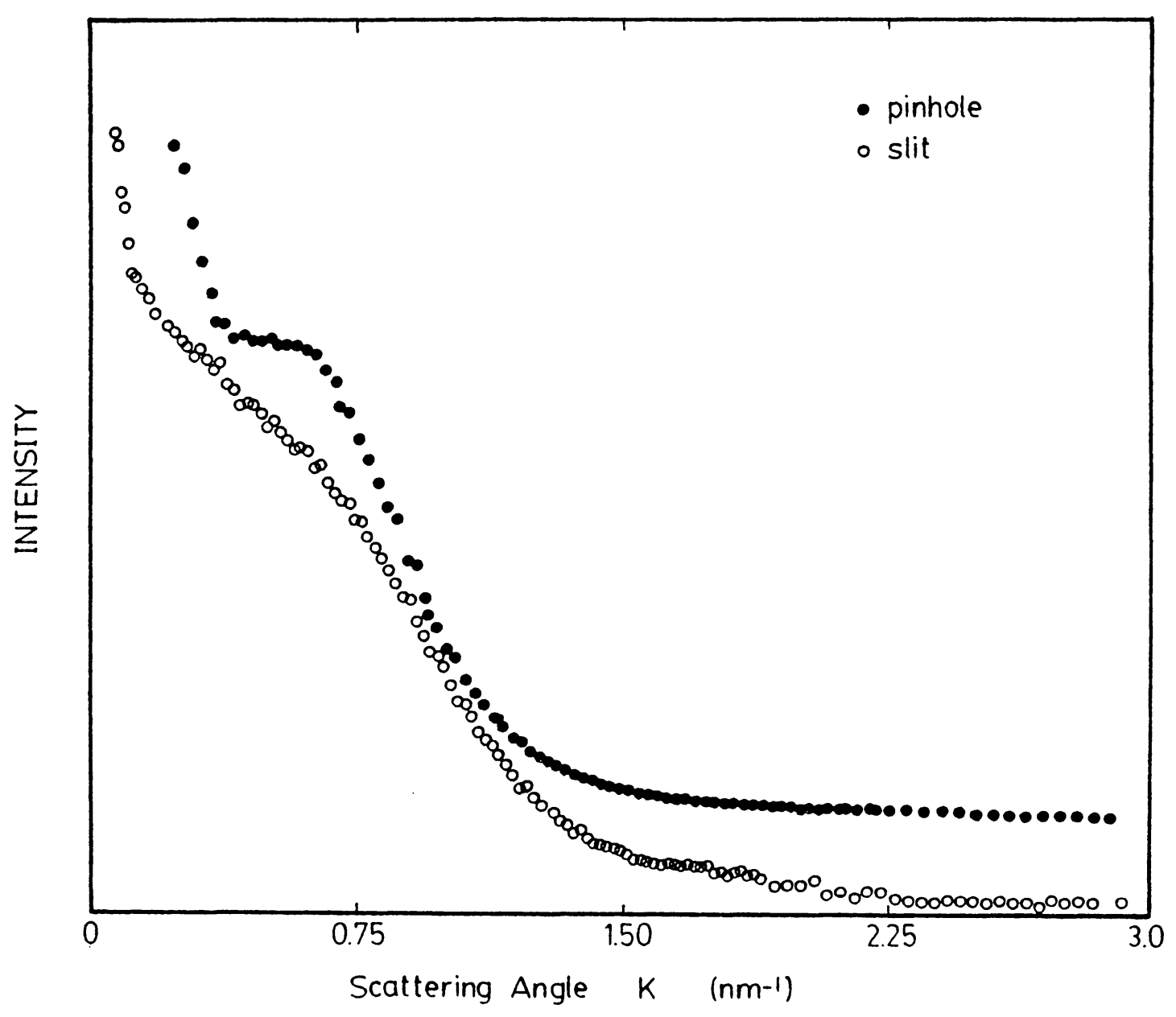

Figure 4.38: Raw slit-smeared and pinhole data for plaque 4 . 


\section{APPENDIX}

Al: Ion Milling of Cold-Fracture Surfaces: Plaques 31135-33-5,6

\section{Introduction}

Recent work on water-blown flexible polyurethane foams led to the suggestion that large-scale polyurea domains are formed during the manufacture of the foam (Rossmy, J. of C.P. 6,319,(1981)). These aggregates are believed to be an order of magnitude larger than domains formed in typical segmented urethane elastomers. In our investigation we proved that there is a high degree of phase separation through DMS and SAXS, and through TEM we have some evidence of the presence of largescale polyurea domains. Cold-fracture surfaces. observed in a SEM reveal 1000-2000 angstrom sized bumps in the surface. The number of these bumps increased with hard segment content. In an effort to learn more about these surface features, the technique of ion milling was applied.

\section{Experimental}

Fracture surfaces approximately one centimeter long were created by scoring the plaque surface and mounting the plaque so that the scored surface was centered between two hose clamps. The plaque was immersed in liquid nitrogen for five minutes and then fractured by rotating the hose clamps apart along the axis created by the scored surface. This long and uniform fracture surface was mounted as a fiber would be mounted for routine ESCA analysis.

A Krotos XSAM800 $x$-ray photoelectron spectrometer was used for the analysis. Argon was used for the ion milling operation. The flow of argon was adjusted so that the vacuum in the system decreased from $10 \star \star(-7)$ torr to $10 * *(-5)$ torr. The argon stream was ionized at a 3 killivolt 
potential and a current of 20 milliamps. The progress of the ion milling operation was followed by ESCA. The ion milled plaques were studied with a scanning electron microscope.

\section{$\underline{\text { Results }}$}

In plaque 5 (4 pph water), the ratio of carbon to nitrogen as determined by ESCA was used to follow ion milling. For plaque 6 (2 pph water), carbon, nitrogen, silicon, and oxygen peaks were monitored. The results are presented in Figures 1 through 4 and Tables 1 and 2 . SEM micrographs were taken of both plaques after roughly 5 and 16 minutes of ion milling (Figures 5 and 6 ). (Figures 22,23 of thesis)

Ion milling is an abrasive or erosive process which may or may not be selective. The rate of erosion may depend on morphological features such as crystallinity, crosslinks, as well as chemical composition. Ion milling causes undefined chemical reactions on the surface and the milling process can change the surface area of the sample. When ESCA accompanies ion milling, ESCA peaks are typically broadened by the undefined chemistry and the integrated intensity changes with changing surface area. The following statements can be made about the data:

1) There is a relatively high concentration of silicon on the fracture surfaces and this concentration diminishes with ion milling.

2) The concentration of oxygen on the surface decreases with ion milling.

3) The area under the nitrogen peak at first increases with milling and then decreases.

4) For plaque 5 the area under the carbon peak increases quickly at the start of the ion milling process and more slowly as the ion milling proceeds. For plaque 6 , an increase in the area under the carbon peak is observed for the first five minutes of milling. After 17.5 minutes of milling the area under the carbon peak has decreased.

5) The $C$ is peak changes shape upon milling. The $C$-O shoulder becomes unresolvable and there is more area in regions due to aromatics and $C=O$. 
The SEM micrographs for plaque 5 show that the unmilled fracture surface is rough with 1000-2000 angstrom bumps. After five minutes of ion milling the surface looks like the unmilled sample with slightly enhanced features. After 12 minutes the surface becomes rougher and after 16 minutes small undulations appear.

The SEM micrographs for plaque 6 show a smooth surface with a few barely resolvable 1000-2000 angstrom bumps. After 5 minutes of milling a faint brainlike texture appears with 2000 angstom continuous bands. The surface texture remains about the same with longer milling times. (The higher contrast at 17.5 minutes is due to the accidental application of over 400 angstoms of Au-Pt.)

\section{Discussion}

The surface features of plaque 5 were possibly enhanced at low milling times. On further milling the isolated bumps that characterized the unmilled surface gave way to a rougher surface of ridges and undulations. ESCA indicated that at low milling times the surface concentration of nitrogen relative to carbon increased. On further milling this ratio decreased. One explanation for this is that the ion milling process does erode away the soft segment more quickly than the nitrogen containing hard segment. After short milling times there is more hard segment on the surface. The hard segment domains begin to protrude from the surface protecting the surrounding soft segment and leaving themselves open to bombardment. This mechanism has been suggested for the milling of spherulites (correspondence with D. W. Dwight). The spheflites are much tougher than the surrounding amorphous polymer at first, but when they begin to protrude too much, they are effectively milled by the ion 
beam.

The fracture surface of plaque 6 did not contain the high concentration of bumps observed in plaque 5. Fracture surfaces exposed to five minutes of milling showed a brainlike surface texture. Further milling did not seem to change the surface texture. The brainlike texture resembles TEM micrographs of well phase-separated urethane and SBS copolymers. These micrographs are, however, of surface features and could possibly be due to surface melting. The plaques did heat up to $65 \mathrm{C}$ during milling, but this measurement involves conduction through the polymer to the sample holder and does not reflect surface temperatures.

\section{Conclusions}

The ion milling of plaque cold fracture surfaces was carried out hoping that the milling process and ESCA would help confirm the presence and define the composition of the relatively large domains observed by TEM. The milling operation, though possibly selective, did not enhance the surface features enough to show the presence of two phases. When low hard segment material was milled a brainlike surface texture was generated, possibly due to surface melting. Chemical analysis of the milled surfaces reflected the undefined chemistry that occurs during the milling process. This first attempt at ion milling does not reveal great potential for ion milling in our work with polyurethane foams. 
Table 1: Ion Milling as followed by ESCA, Plaque 31135335

Integrated intensities were adjusted to reflect correct stoichiometry. Data are normalized to $\mathrm{C} 1 \mathrm{~s}$ peak at 0.0 milling time.

$\begin{array}{clc}\begin{array}{c}\text { time } \\ (\min )\end{array} & C 1 \mathrm{~s} & N 1 \mathrm{~s} \\ 0 & 1.0 & - \\ 3 & 1.23 & 0.030 \\ 6 & 1.29 & 0.039 \\ 11 & 1.38 & 0.040 \\ 16 & 1.42 & 0.036\end{array}$

Table 2: Ion Milling as followed by ESCA, Plaque 31135336

Integrated intensities were adjusted to reflect correct stoichiometry. Data are normalized to $\mathrm{C} 1 \mathrm{~s}$ peak at 0.0 milling time.

$\begin{array}{rllll}\begin{array}{c}\text { time } \\ (\min )\end{array} & \text { C1s } & \text { N1s } & \text { Si2p } & \text { O1s } \\ 0.0 & 1.0 & 0.022 & 0.19 & \\ 5.0 & 1.48 & 0.027 & 0.075 & 0.34 \\ 17.5 & 1.30 & 0.016 & 0.035 & 0.13\end{array}$


Figure 1

Ion-bombardment and ESCA analysis of cold fracture surface

Series: $31135-33$

Sample: Plaque 5, 4 pph water, TDI index 110

Wide scan, sputtering time 0.0 minutes

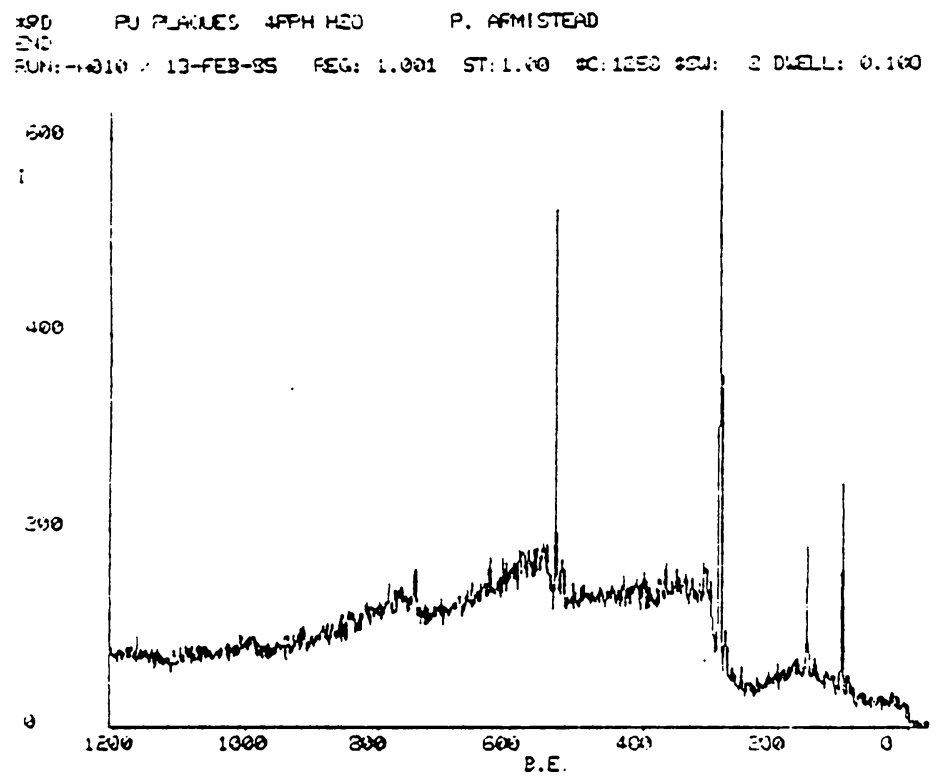

Wide scan, sputtering time 16.0 minutes

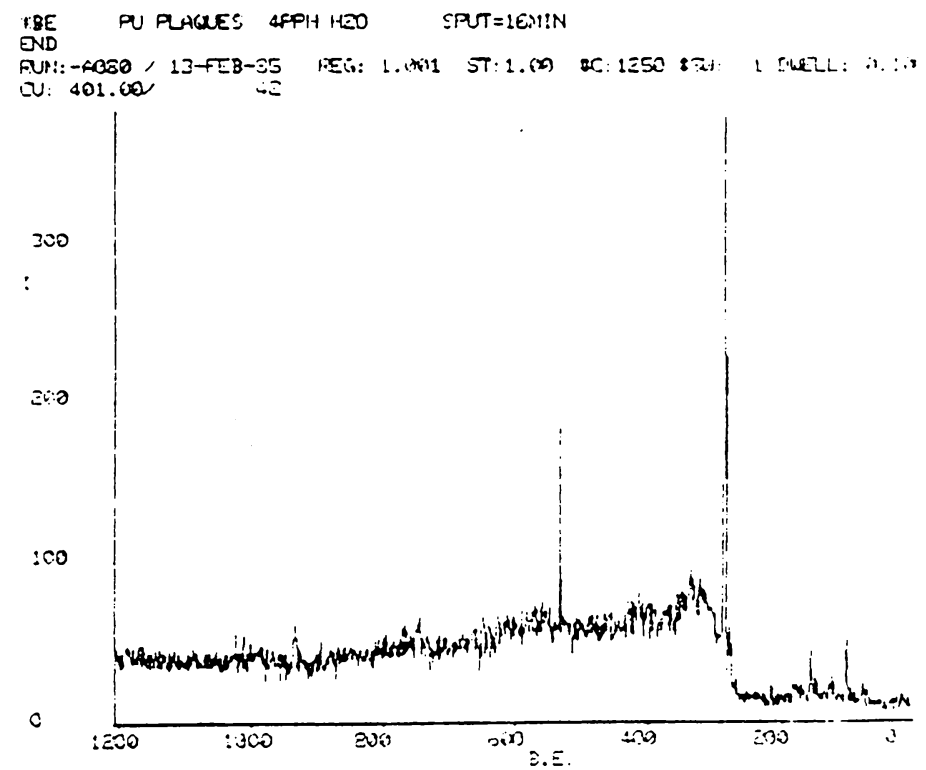


213

Figure 2

Ion milling and ESCA analysis of cold fracture surface

Series 31135-33

Sample Plaque 5, 4 ph water, TDI index 110

Cis peak, milled for 0.0 minutes

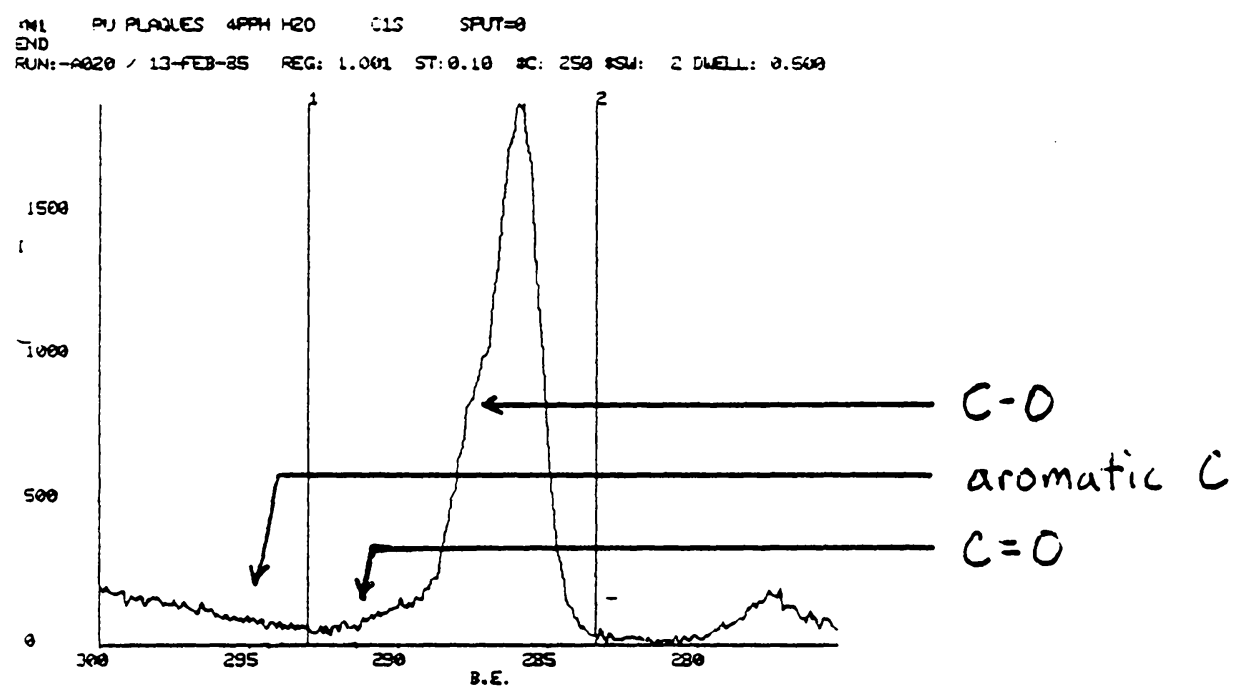

Cis peak, milled for 16.0 minutes

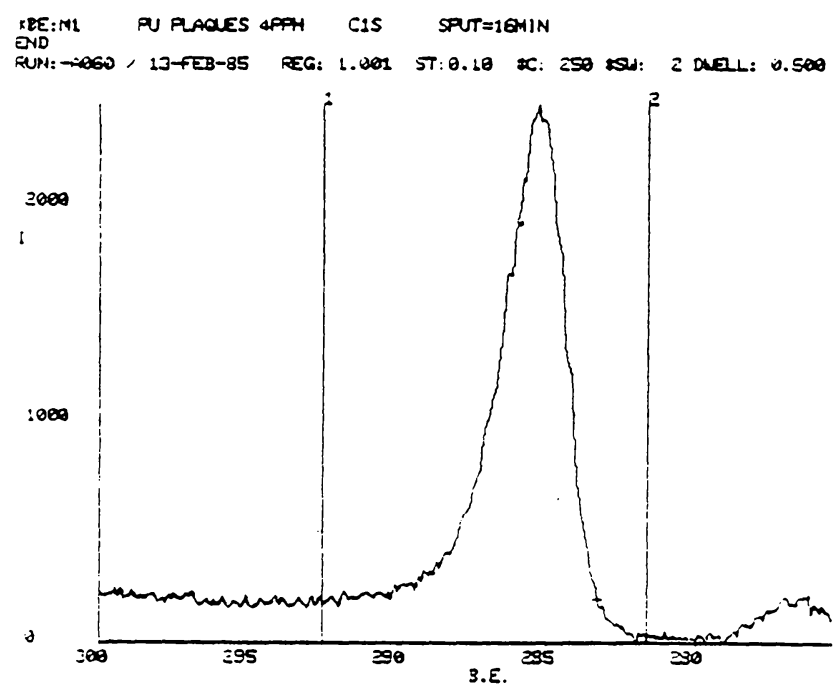


214

Figure 3

Ion milling and ESCA of cold fracture surface

Series: 31135-33

Sample: Plaque 6, 2 ph water, TDI index 110

Cis peak before and after milling
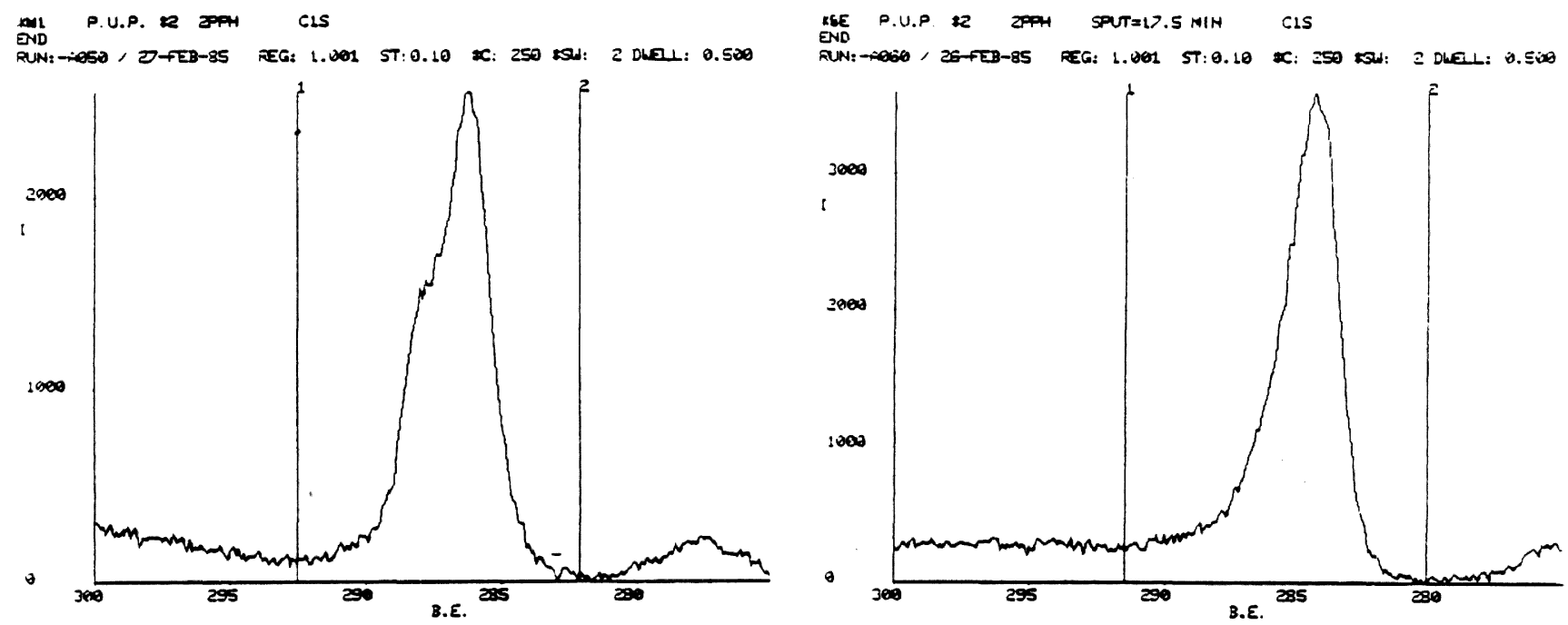

N1s peak before and after milling
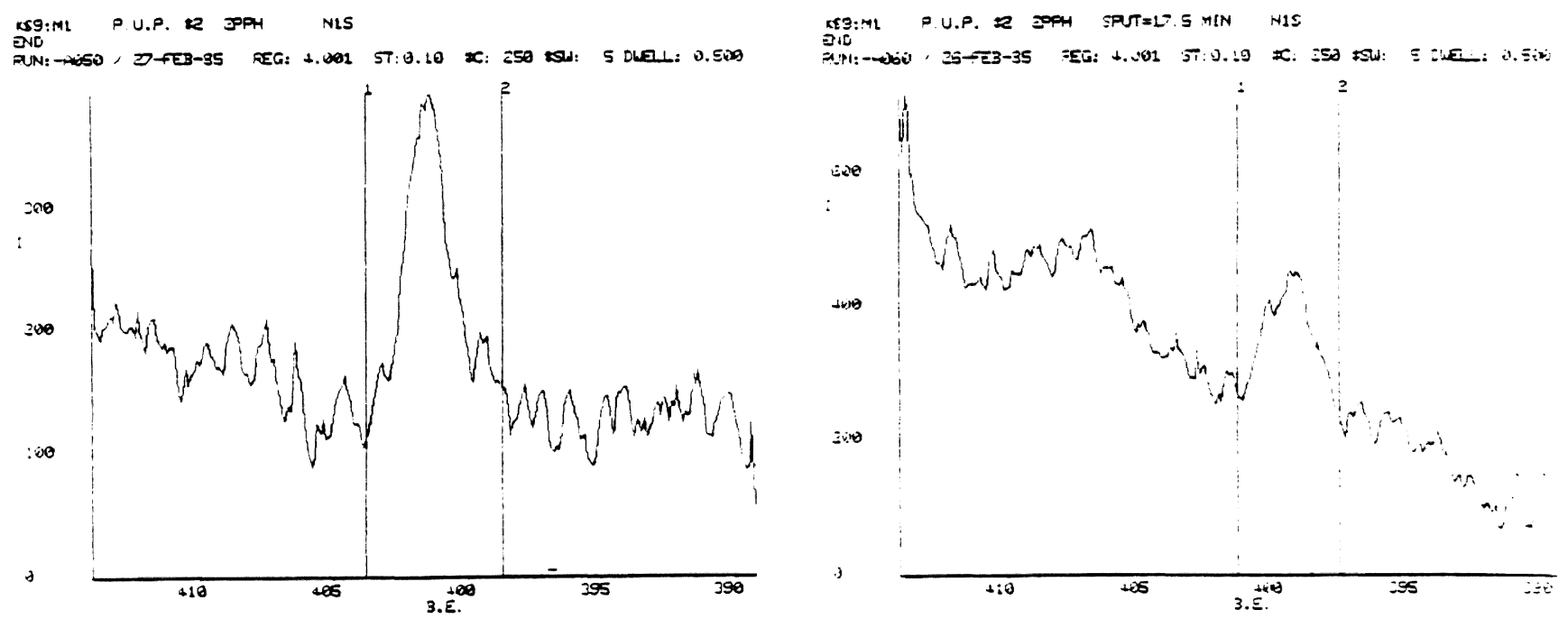
Figure 4

Ion milling and ESCA of cold fracture surface

Series: 31135-33

Sample: Plaque 6, 2 pph water, TDI index 110

Si2p peak before and after milling
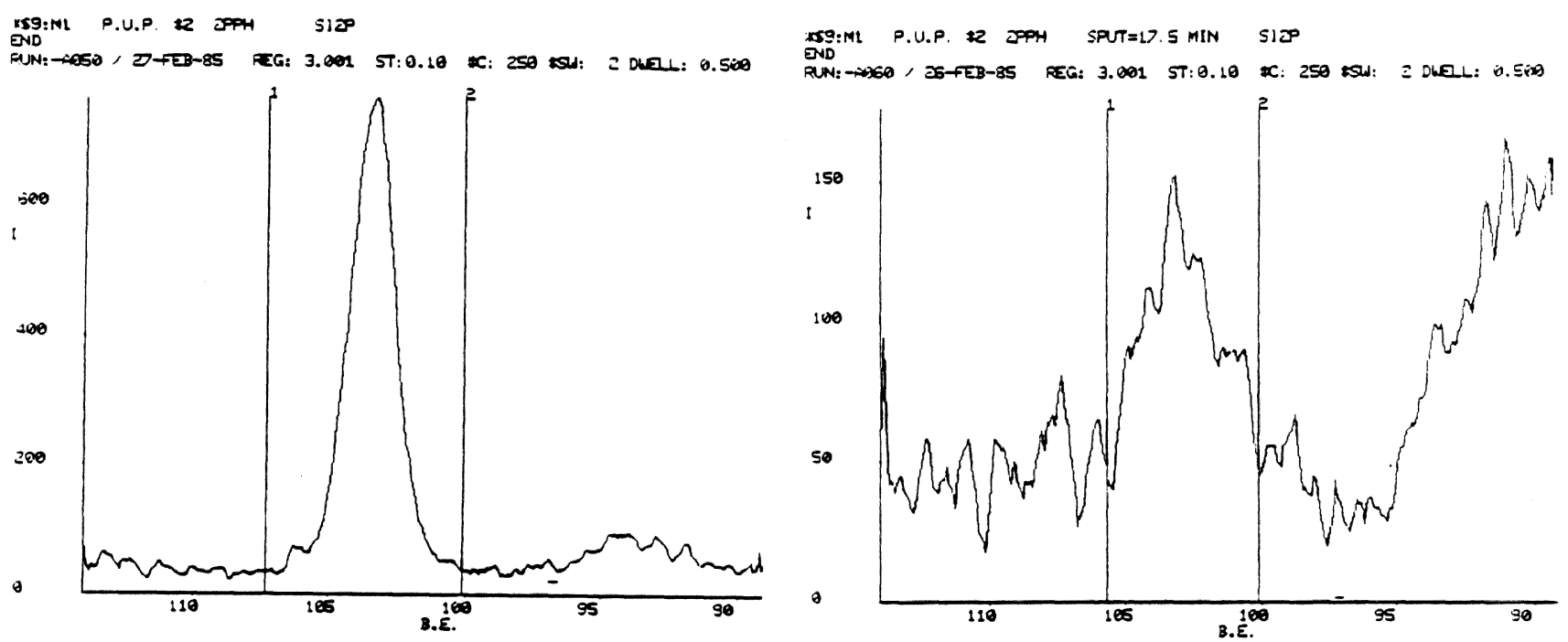

$01 \mathrm{~s}$ peak before and after milling
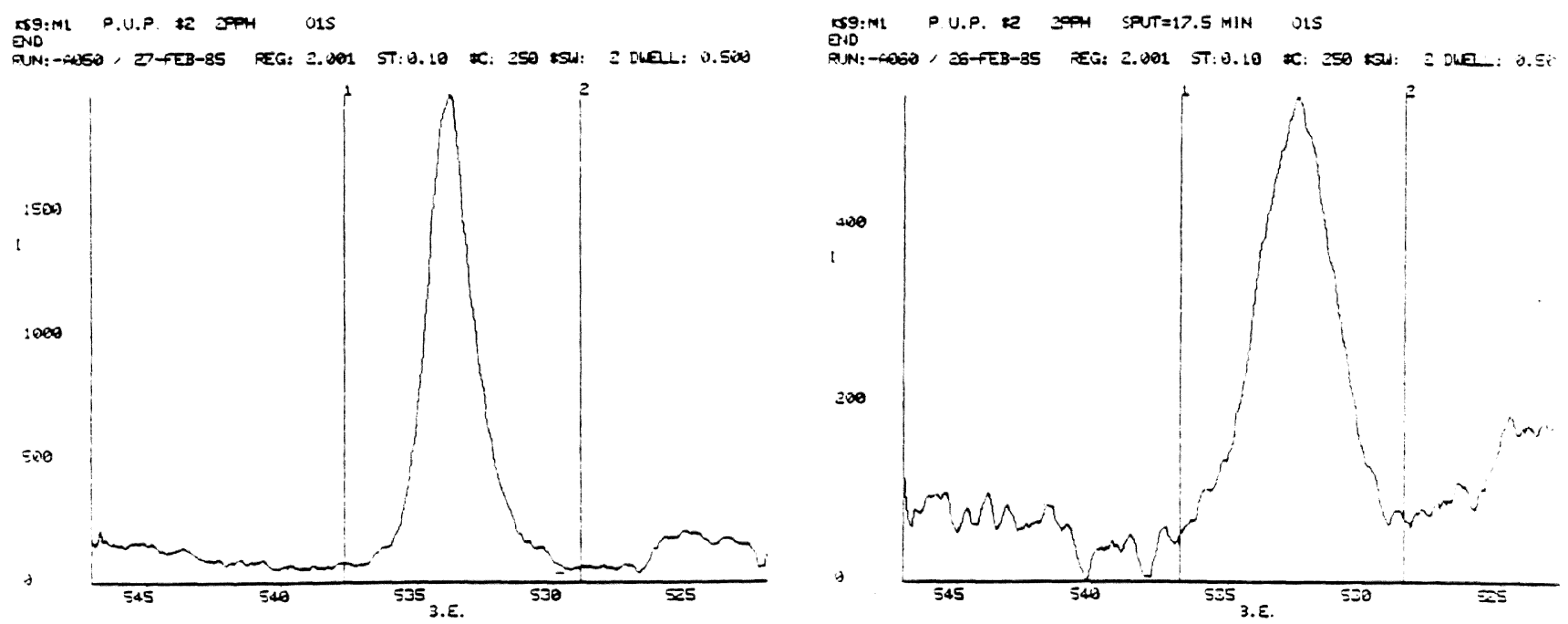


\section{APPENDIX AZ: \\ Dynamic Mechanical Spectroscopy Study of FleXible Urethane FoAm}

\author{
R. B. Turmer ano H. L. SPell \\ Texas Applied Science and Technology Laboratory \\ Dow Chemical U.S.A. \\ Freeport Texas 77541
}

\author{
G. L. WILKES \\ Polymer Materials \& Interfaces Laboratory \\ Department of Chemical Engineering \\ Virginia Polytechnic Inst \\ Blacksburg, VA 24061
}

\section{INTRODUCTION}

W

hile many studies of the physical properties of flexible polyurethane foams have been discussed within the literature, relatively little work has been reported which utilizes the dynamic mechanical spectrum (DMS) of the foamed material. G. Woods [1] has suggested that the base polymer of flexible polyurethnne low density foams may be somewhat analogous to polyurethane elastomers, i.e.. they may be considered as block copolymers with hard polyurea blocks linked via urethane groups through flexible polyether chains. In fact. the proof of domain or microphase separation has recently been demonstrated by one of us using the method of small angle X-ray scattering [2].

The object of the present work is to relay some initial observations as obtained from the dynamic mechanical properties of a systematic series of foams over a wide range of temperature at a fixed frequency. Testing was done on the foamed specimen as well as a compression molded plaque of the same material. The information obtained. combined with our other studies utilizing electron microscopy and small angle X-ray scattering techniques, serve to characterize the morphological structure in the foam. It is hoped that this approach will help in assessing

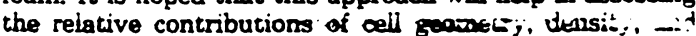
base polymer properties to the overall physical properties of the foam.

\section{EXPERIMENTAL}

The dynamic mechanical experimeists described here were done on either a Rhecmetrics Model 605 Mechachanical Spectrometer or a Rheometrics Model 7700 Dynamic Spectrometer. Foam samples were tested in a parallel place mode during which a cylinder of foam is subjected to an oscillatory shear deformation. The frequency of deformation was $\mathrm{H} \mathrm{H}_{2}$, while the strain was approximacely $1 \%$. Normally, the temperature range was from -160 to $300^{\circ} \mathrm{C}$ with points recorded every five degrees after a thermal "soak time" of two minutes.

The sample was cut from the slabstock with a high speed cork borer. Dimensions were $25 \mathrm{~mm}$ diameter by $25 \mathrm{~mm}$ height. The foam cylinder was mounted on disposable plates by applying General Electric Silicone High Temperature Gasket Sealant to the plate surfaces and cylinder ends. The sample was compressed slightly while mounted in the instrument and the sealant allowed to dry for $\mathbf{3 0}$ minutes.

\section{DISCUSSION}

The DMS spectrum of foam / 3 shown in Figure 1 is very similar to that of a well phase segregated segmented urethane elastomer. The transition from glass to rubber is fairly sharp with the glass transition of the polyol occurring very near the limiting value exhibited by a model structure [3]. The rubbery plateau is nearly flat and extends to the temperature normally assigned to disruption of the microdomains of the urea hard blocks and possibly degradation. The fact that the base polymer of the foam has a fairly flat temperature insensitive modulus offers indirect support for a virtual and/or covalent crossliniked network More specifically, the position. sharpness, and intensity of the polyol glass transition argues for a well segregated domain structure with little phase mixing.

A comparison of the DMS data of the foam with the data of the same material which has been compression molded indicates that there are some chemical and/or morphological changes that have occurred during this latter thermal event. For example, the glass transition of the polyol phase intensifies and shifts to a lower temperature (see Figure 1b). (Glass transition values for all four foams and corresponding plaques are presented in Table 1.) The rubbery plateau flattens and shows greater thermal stability. These observed changes suggest that phase organization is enhanced by the molding operation, aithough the overail modulus is reduced siightly.

Previous workers $[4]$ have suggested that allophonate and/or biuret linkages could be present and affect the mechanical behavior of foams subjected to increasing temperature. Since both of these linkages are temperature labile. their presence should be reflected in modulus changes with temperature rise. Other than the spurious appearance of a minor loss transition at about $100^{\circ} \mathrm{C}$ in two of 

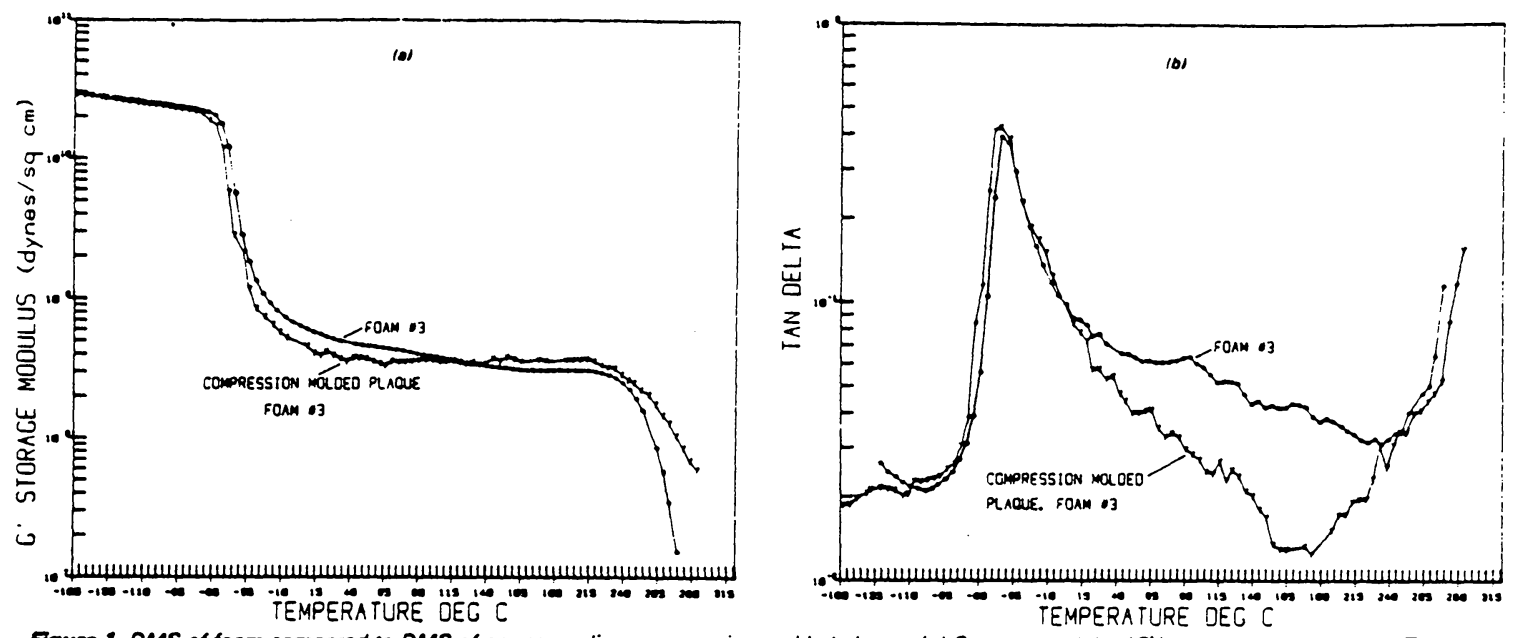

Figure 1. DMS of foam compared to DMS of comesponding compression molded plaque. (a) Storage modulus (G) versus temperature, (b) Tan deta versus temporoture.

the foams, no evidence was observed to substantiate the presence of thermally unstable linkages. Other possible chemical crossiniks including isocyanate trimers will be covered in future reports.

In order to compare DMS data of foams and plaques and for that matter to intercompare data among foam samples. the DMS spectral values were normalized to that of a typical polyurethane plaque in the glassy state at $-160^{\circ} \mathrm{C}$ $\left(G^{\prime}=3 \times 10^{10}\right.$ dynes $\left./ \mathrm{cm}^{2}\right)$. Figure 2 shows spectra of a series of foams as they were originally recorded. The moduli of the four foams in the glass region order directly with increasing density as expected. Other treatments of the original data such as factoring out density have beén attempted and will be considered later. Correlations of some of the spectral data with volume fractions applying the law of mixtures may be worthwhile.

Figure 3a shows the normalized DMS spectra of a series of four foams in which the water concentration is varied (i.e. density). The storage modulus, which is a measure of stiffness, increases as expected with decreasing foam density and increasing hard phase content, i.e. increasing TDI. Presumably as the water-isocyanate reaction increases, i.e. with larger proportions of water, greater amounts of a urea rich phase are formed. These are comprised mainly of aromatic urea polymeric units with little or no incorpora- tion of polyols through carbamate linkages. Hence, as the density of the foam goes down, the filler-like urea rich microphases produce a rise in modulus.

It is noted also that the softening of the hard segments. whether it is the thermal induced flow or degradation of crosslinked material, occurs at slightly higher temperatures with increasing urea group concentrations (see Table 1). It is expected that the increased reaction temperature of the foams with greater amounts of water should favor phase organization [1]. The glass transition of the polyol which should be the most sensitive measure of extent of phase segregation does not show an ordered variation (see Table 1).

Of interest is the observation that the slope of the storage modulus in the use temperature range decreascs with decreasing density of the foams (Table 1). The foam of highest density exhibits a DMS spectrum with the most rubber-like characteristics (see Figures $3 a$ and $3 b$ ). The intensities of the glass transition of the polyol phase show a concomitant decrease with decrease of polyol content (Figure 3b). Additional efforts toward quantitating these data are in progress. The relation of modulus to density for the set of foams is shown graphically in Figure 4. Change of modulus $\left(G^{\prime}\right)$ of the foams in the glassy stace shows a monotonic increase with increase in density. This response

Table 1. Oynamic mochanical data of serias 1 to 4 polyurothene toams and corresponding ploques.

\begin{tabular}{|c|c|c|c|c|c|c|c|c|}
\hline \multirow[b]{3}{*}{ Sample } & \multirow[b]{3}{*}{ Density (lbs/tt') } & \multicolumn{6}{|c|}{ Transitions ${ }^{\circ} \mathrm{C}$} & \multirow{3}{*}{$\begin{array}{l}\mathrm{G}^{\prime} \text { Ratio } \\
125 / 20^{\circ} \mathrm{C}\end{array}$} \\
\hline & & \multicolumn{5}{|c|}{ Tg (ol) } & \multirow[b]{2}{*}{$T_{\text {herd }}$} & \\
\hline & & $(\tan d)$ & $\max$ & $\tan$ & $\delta$ & $\mathbf{G}^{-}$ & & \\
\hline 1 & 2.85 & 0.58 & $(0.68)$ & -40 & $(-43)$ & $-52(-54)$ & 235 & 0.93 \\
\hline 2 & 1.92 & 0.51 & $(0.59)$ & -38 & $(-40)$ & $-47(-50)$ & 245 & 0.84 \\
\hline 3 & 1.43 & 0.38 & $(0.43)$ & -38 & $(-42)$ & $-48(-52)$ & 243 & 0.58 \\
\hline 4 & 1.24 & 0.31 & $(0.35)$ & -41 & $(-42)$ & $-48(-49)$ & 250 & 0.65 \\
\hline
\end{tabular}

Note: Data trom compression molded plaques in parenthess. 


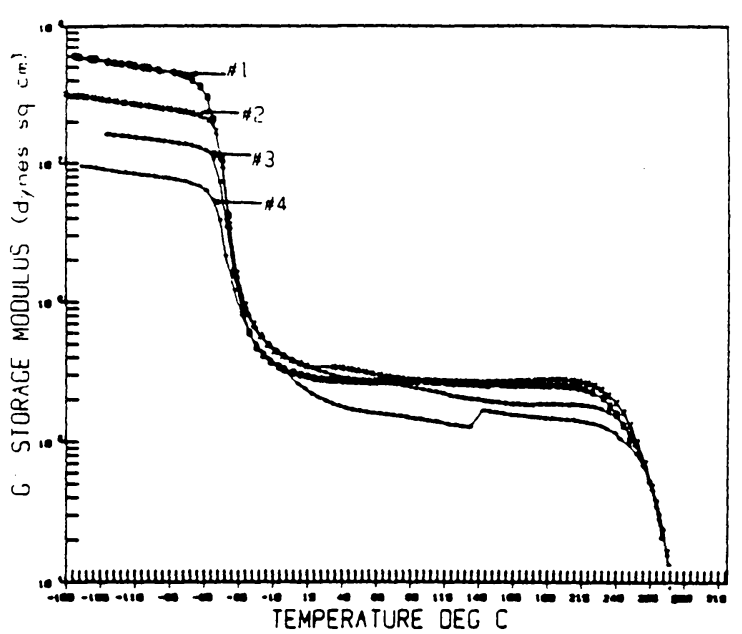

Figure 2 DMS of series of foams with varying densities.

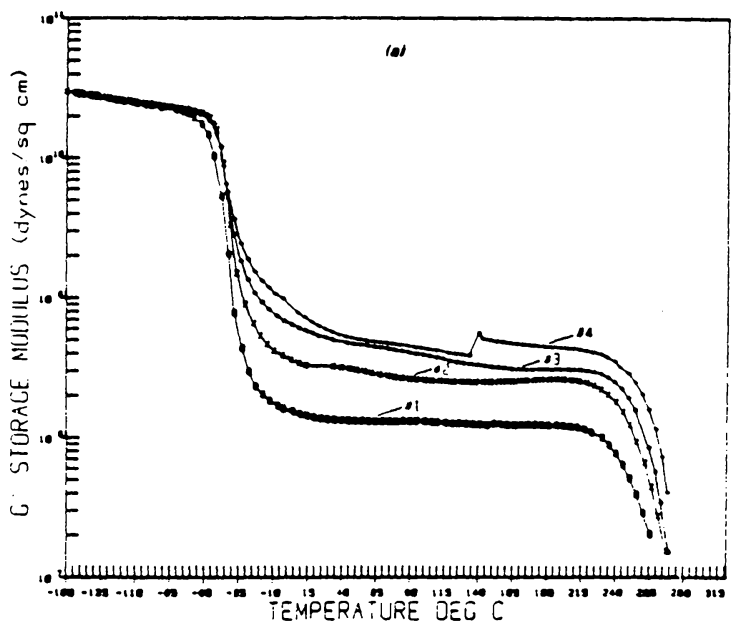

Figurs 3. OMS dato from figure 2 normalized to glassy stote at $-160^{\circ} \mathrm{C}$. remperature.

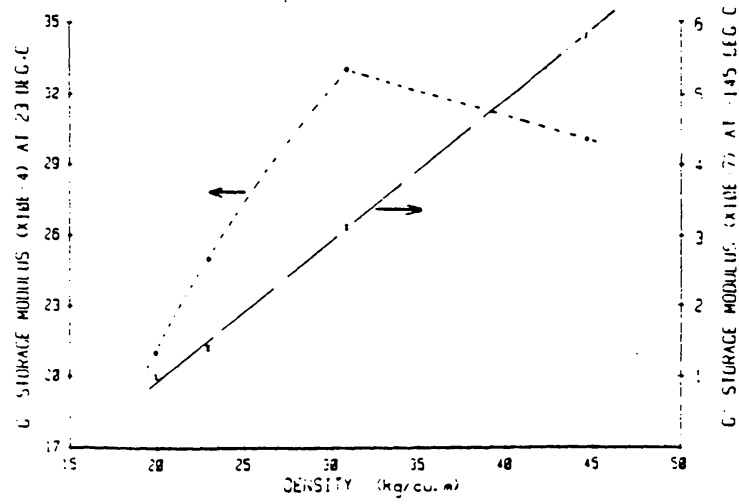

Figure 4. Storage modulus at $23^{\circ} \mathrm{C}$ and $-145^{\circ} \mathrm{C}$ versus densin of the toams. is expected since in the glassy state all phases and struc. tures react the same to the testing, and the modulus increase with density is mainiy caused by the increasing amount of glassy material between the test plates. On the other hand, the room temperature modulus is affected both by density and morphological organization. Other studies that have been initiated include foam anisotropy [5]. Also, transmission electron micrographs of cross sections of cell windows and struts and small angle $X$-ray scattering results will be covered.

\section{CONCLUSIONS}

This work has demonstrated that DMS, because it is both an analytical tool and a testing method, provides qualitative and quantitative data to characterize the base polymer in a urethane foam.

Comparisons of the dynamic mechanical spectra of the compression molded plaques (from foams) with the spectra of the corresponding foams indicate morphological changes have occurred as a result of the modling conditions, i.e., (a)

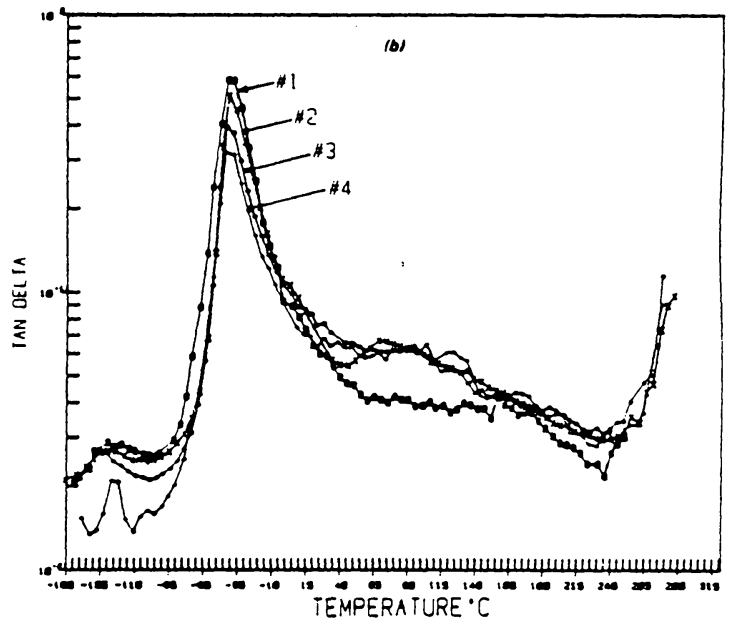

(a) Storage modulus $(G$ ) versus temperature, (b) Tan defto versus

The $T_{8}$ of the polyoi phase intensified and shifted to a lower temperature; (b) The rubbery plateau flattened and showed greater thermal stribility. These observations suggest phase organization was enhanced by the molding operation.

The flat. nearly temperature insensitive modulus offers support for an elastomeric material with a well ordered vir. iual and/or covalent crosslink network. The position. sharpness and intensity of the poly glass transition argues for a relatively pure polyol phase. Its glass transition is practically invariant at about $-40^{\circ} \mathrm{C}$ for the series of foams. suggesting that the relative goodness of phase separation is quite similar. The storage modulus $\left(G^{\prime}\right)$ or stiffness of the polymer increases concomitantly with decreasing density in the foam formulations. The formation of a higher proportion of rich urea phases. which act as a solid filler, could ac. count for the stiffening of the polymer. Results from transmission electron microscopic examinations of thin sections of the series of samples support this theory. The high 
temperature stability of the polymer also increases slightly with rise in water content in the formulation.

Measurements of the modulus at low temperatures (glassy state) show a monotonic increase with increase in density among the series of samples.

\section{ACKNOWLEDGEMENTS}

The authors wish to thank J. W. Wilchester. L. D. Hendershott. D. J. McDowall and Don Germano for working out the details in sample preparation and instrument operation during the course of this study. We also want to thank L. C. Mulford and P. Pham for sample preparation.

\section{BIOGRAPHIES}

\section{H. L. Spell}

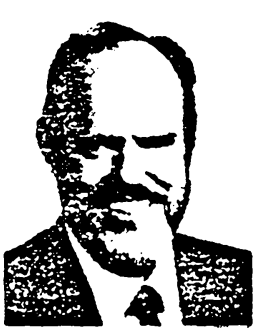

Presently a Sr. Associate Scientist. Spell began his Dow career in 1952 and worked until 1973 in the field of molecular spectroscopy including ultraviolet, infrared and Raman. For the next six years he worked with the applications of $X$-ray photoelectron spectroscopy (ESCA) to problems related to sur. face chemistry. During the last four years, he has been involved in developing new methods for poly. mer testing and characterization. He has published about 25 papers and presented over 40 papers to national and international meetings. Harry obtained the B.S. degree in physics at Southwestern Louisiana University and the M.S. in chemistry from Texas A\&M University.

\section{REFERENCES}

1. G. Woods, Flexible Polyurethane Foams, Chemistry and Technology. Applied Science Publishers, London p. 58 (1982).

2. L. Wilkes. S. Abouzahr and D. Radovich. J. Cell Plast.. 19 (4), 248-54 (1983).

3. R. B. Turner and C. P. Christenson, to be published in Polymer Engineering and Science.

4. J. H. Saunders and K. C. Frisch. Polyurethanes: Chemistry and Technology of High Polymers. Wiley Interscience. New York (1962).

5. N. C. Hilyard, Mechanics of Cellular Plastics, Macmillan Publishing Co., New York (1982).

R. B. Turner

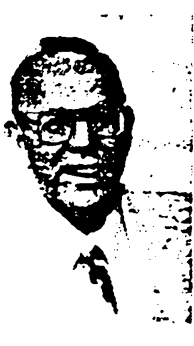

Bob has been working in fundamental polyurethane research for 15 years and is responsible for that group. He has worked in the foam and elastomer area. Prior to that he was involved in polyethylene and chlorinated solvent research. He began his Dow career in 1960 and has published and presented over 52 papers. He is a graduate of Texas A\&M University.

\section{G. L. Wilkes}

Garth is codirector of the polymer Materials and Interfaces Laboratory at Virginia Polytechnic Institute in Blacksburg. Virginia. He is a chair professor in the Chemical Engineering Department. His interests are in the structure property behavior of polymeric materials. 
FTIR STLIDY OF SLABSTTOCK POLYURETHANE(UREA) FOAMS AND THEIR CORRESPONDING COMPRESSION MOLDED PLAQUES (PRELIMINARY REPOET) by M. A. Harthcock

\section{INTRODUCTION}

The study of polyurethane foams using infrared spectroscopy can be most vaiuable in elucidatirg the molecular structure that influences the physical and mechanical properties of the foams. several papers have been presented in the general scientific literature on the infrared study of foam formation.il-5;

The series of polyurethane foams studied here either vary water/isocvanate or tin catalyst concentration. This allows us to study the effect these two variables have on the molecular structure and properties of the final product. The polymerization reaction can lead to a variety of structural features:

(1) Formation of urea linkages

(2) Formation of urethane linkages

(3) Dimerization and/or trimerization of isocyanate groups

(4) Formation of carbodiimides

(5) Formation of allophanates and/or biurets

and (6) Formation of acid groups

The intent of the work presented here is to obtain an understanding of the effect on molecular structure of various concentrations of water (and corresponding isocyanate, T-80, concentration) and tin catalyst, T-9. Also, the effect on the molecular structure on compression molding the foams to form plaques will be considered.

\section{EXPERIMENTAL}

Infrared spectra were collected on a Digilab FTS-10 Fourier transform infrared spectrophotometer equipped with a germanium coated cesium iodide beamsplitter and a triglycine sulfate (TGS) detector. The spectra were collected using a Harrick TMP-220 internal reflection unit. A KRS-5 prism was used and the angle of incidence between the infrared radiation and the prism was $50^{\circ}$. The spectra were collected at $8 \mathrm{~cm}-1$ resolution and 2048 scans were collected for signal averaging.

The foam and compression moided foam samples were provided by R. B. Turner of Urethane and Oxide Polymers Research. The compression itolded plaques that were supplied were made by pressing the foams with 250 tons of pressure at $204^{\circ} \mathrm{C}$ for 10 minutes. Table I gives the formulation of the foams.

\section{RESULTS AND DISCUSSION}

For the foams and compression molded plaques the regions that provide a considerable amount of information about the molecular structure are the isocyanate (NCO), $2270 \mathrm{~cm}-1$, and the carbonyl, $1800-1600 \mathrm{~cm}-1$, regions. However, this later region is complicated due to the wide variety of carbonyls that can exist in the foam and compression molded plaques. The types of carbonyls that are possible and their generalig accepted frequency or frequency range(s) are given in Table II.

Residual NCO content in foams and compression molded plaques (varving water and isocvanate concentration). The residual isocyanate remaining in the foam and compression molded plaques can be determined on a relative basis between the samples using the $2270 \mathrm{~cm}-1$ NCO stretching vibration band. Fiqure 1 presents a plot of the parts of water by 
weight versus various absorbance ratio bands involving the isocyanate band. The trend that is apparent is that with increasing water and isocyanate concentration, the amount of free isocyanate increases. The effect is not as dramatic in the compression molded plaques but does exist. Possible reasons for the residual isocyanate could be:

(1) Incorrect stoichiometry for the foan reaction which becomes more obvious as the water and isocyanate concentration is increased.

and (2) Isolation of isocyanate groups due to a changes that occur as the water and isocyanate concentration is increased.

Regardless of the reason for the residual isocyanate, the fact that there is some residual isocyanate can effect the properties of the foams. Also upon compression molding the foams, the residual isocyanate may react in some fashion to influence the final molecular structure of the compression molded plaque.

Structural information from the carbonvl region for foams and compression molded elaques (varying water and isocyanate concentration). Figure 2 presents the spectra for the foams with varying water and isocyanate concentration in the $1800-1300 \mathrm{~cm}-1$ region. The spectra are indicative of that for all the foams studied. Two assignments are easy to make from the spectra. The bands at $1642 \mathrm{~cm}-1$ and $1730 \mathrm{~cm}-1$ can be assigned to the carbonyl stretching vibration of hydrogen-bonded urea and free urethane groups, respectively. These two bands normally appear as the most intense carbonyi stretching bands in spectra of the polyurethane (urea) foams. Several other bands are observed

(1) A shoulder in the $1720-1708 \mathrm{~cm}-1$ region

(2) A band at $1696 \mathrm{~cm}-1$

(3) A band at $1685 \mathrm{~cm}-1$

(4) A band at $1675 \mathrm{~cm}-1$

and (5) A band at $1656 \mathrm{~cm}-1$

The assignment of the latter three bands will not be considered because the "realness" of these bands is uncertain. The assignment of the band $(\mathrm{s})$ in the $1720-1.708 \mathrm{~cm}-1$ region and at $1695 \mathrm{~cm}-1$ is a more difficult task.

The band(s) in the $1720-1708 \mathrm{~cm}-1$ region can be assigned to several carbongl vibrations listed in Table II. The five possibilities are carbonyls in carbamic acid, TDI trimer, allophanate, biurets, and hydrogen bonded urethane linkages. Carbamic acid is an unstable species in the foaming reaction ( 6 ) and has been observed by Bailey and Critchfield (5) to appear early in the reaction.

$$
\begin{aligned}
& \mathrm{R}-\mathrm{N}=\mathrm{C}=\mathrm{O}+\mathrm{H}_{2} \mathrm{O} \rightarrow \mathrm{R}-\stackrel{\mathrm{H}}{\mathrm{I}} \stackrel{\mathrm{O}}{\mathrm{N}}-\mathrm{C}-\mathrm{O}-\mathrm{H} \rightarrow\left(\mathrm{R}-\mathrm{NH}_{2}\right)+\mathrm{CO}_{2} \\
& \text { carbamic acid } \downarrow \mathrm{R}-\mathrm{N}=\mathrm{C}=\mathrm{O} \\
& \mathrm{R}-\mathrm{N}-\mathrm{C}-\mathrm{N}-\mathrm{R} \\
& \begin{array}{lll}
1 & \| & 1 \\
\mathrm{H} & \mathrm{O} & \mathrm{H}
\end{array}
\end{aligned}
$$


lhe carbonyl stretching frequency for carbamic acid comes at about $1710 \mathrm{~cm}-1$. It is unlikely that a significant amount of carbamic acid groups remain after the foaming reaction and contribute significantly to this shoulder in the $1720-1708 \mathrm{~cm}-1$ reqion.

Allophanates and biurets are formed by reaction of secondary urethane nydrogens with isocyanate to form a crosslink between polymer chains in the form of a covalent bond. (6)

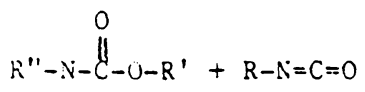

$\mathrm{H}$<smiles>[R]NC(=O)N([R]O[R])C(=O)O</smiles>

allophanate

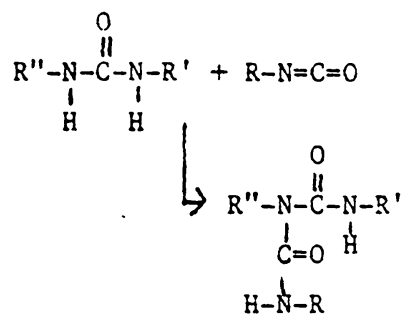

biuret

The biurets are more likely to form because of the greater reactivity of ureas to isocyanate than urethanes. The presence of bands between 1650 and $1700 \mathrm{~cm}-1$.would support the presence of some of these zrosslinks as these bands are secondary bands to a more intense band that occurs in the 1751-1721 and 1720-1690 cm-l regions for allophanates and biurets, respectively. The frequency would more closely be that of a biuret. These species are thermally unstable and may form and dissociate during the foaming reaction. However, some covalently bonded crosslinks may still exist at the end of the foaming reaction. The formation of TDI trimer (a substituted isocyanurate) occurs by the following reaction:

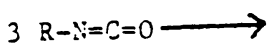

Iwo bands at about 1705 and 1410<smiles>[R]n1c(=O)n([R])c(=O)n([R])c1=O</smiles>

A substituted isocyanate (trimer) bands may support the presence of the trimer.

Finally, is the possibliity that hydrogen bonding can occur (7-9). The absorption in the 1720-1708 cm-1 region can be assigned to hydrogen bonding of urethane carbonyls to $\mathrm{NH}$ groups. It is recognized that phase segregation does occur in foams. (10) and as a result. a significant amount of hydrogen bonding can occur between carbonyls and lit groups. 
It is not likely that the band in the $1720-1708 \mathrm{~cm}-1$ region is due solely to one of these types of carbonyls but is due to a combination of several of these species. Although it is difficult to make an assignment of the band in the 1720-1708 cm-l region, the band can be considered as an indication of the crosslinking in the foam. As we have observed, the probable assignments of this band are due to stretching vibrations of carbonyls incorporated in a crosslink of some kind.

The $1695 \mathrm{~cm}-1$ band can be assigned to two possiblities: free urea groups and/or the secondary band of a allophanate or biuret functionality. Both of these are viable assignments for the $1695 \mathrm{~cm}-1$ band.

The trend observed in the spectia of the foams as the water and isocyanate content is increased is as would be expected. The amount of urea versus urethane linkages increases. Figure 3 shows a plot of parts by weight of water and various absorbance band ratios for the urea carbonyl ( $1642 \mathrm{~cm}-1$ ) and urethane carbonyl (1732 cm-l) bands. one can particularly observe the increase in urea concentration from the plot of Aurethane/Aurea versus parts by weight of water. No other obvious trends were observed from varying the water concentration.

The infrared spectra of the compression molded foams are given in Figure 4 in the 1800-1300 cm-1 region. Figure 5 gives the spectra in this region comparing the foams and compression molded plaques.

The compression molded plaques give similar spectra in the $1800-1300 \mathrm{~cm}-$ region as the foams. However, several of the bands in the 1700-1650 cm-1 region are not as intense or absent from the spectra of the compression molded plaques. Also the amide band(s) around $1340 \mathrm{~cm}-1$ show a more distinct shape. This is not as dramatic for the 33-3 and 33-4 compression molded plaques. The change, although subtle, may be due to breakdown of weak crosslinks (e.g. allophanates and biurets) upon compression molding of the foams. Also, the compression molding may cause the hard segments to be bette ordered which results in hydrogen bonding between carbonyls and $\mathrm{NH}$ groups and/or a decrease in the distribution of various carbonyls.

From the spectra of the compression molded plaques and the fact that biurets and allophanates are thermally unstable, it may be that bands in the 1720-1700 cm-1 region are due to free urea groups, hydrogen bonded urethane groups and/or trimer. However, once again, the complexity of this region does not allow us to make a definite assignment of the bands. It should also be noted that the decrease in unreacted isocyanate in the compression molded foams may be a result of reaction of the isocyanate with various groups in the material.

Structural information from the carbonyl region for foam and compression molded olaques (tin catalyst. T-9, concentration). Figures 6 and 7 give the infrared spectra in the $1800-1300 \mathrm{~cm}-1$ region for the foam and compression molded plaques with varying tin catalyst, T-9, concentration, respectively. The foams with higher concentration of the catalyst shows a iower concentration of urethane groups. However, the difference between the samples is not great and may only be due to differences in experimental error in determining relative urethane content. Therefore, the effect the catalyst concentration has on the molecular structure seems to be minimal. The plaques show no trend when tin catalyst is varied. The 33-6 compression molded plaque shows a significantly larger urethane band at $1732 \mathrm{~cm}-1$ when compared to the urea bands than any of the other samples. 


\section{CONCLUSION}

Fourier transform infrared spectroscopy has been used to study a series of polyurethane foams and the compression molded foams in which the water/isocyanate or tin catalyst, T-9, was varied in the formulation. Several conclusions can be drawn from the study and they are listed below.

(1) As the water concentration increases, the amount of urea linkages increases.

(2) As the water concentration increases, the amount of unreacted isocyanate increases.

(3) The compression molded plaques show less unreacted isocyanate than the corresponding foam.

(4) The urea versus urethane content appears to remain constant upon compression molding of the foams.

(5) Changes are observed in the carbonyl region upon compression molding of the foams. This may be due to better ordering of the hard segments and breakdown of some of the weak crosslinks (e.g. biurets and allophanates).

and (6) Increasing the tin catalyst, $T-9$, shows only minor changes in the urea versus urethane linkages and therefore the tin catalyst seems to have little effect on the molecular structure.

\section{ACKNOWLEDGEMENTS}

Thanks to $I$. D. Hendershott who assisted in recording some of the infrared spectra and H. L. Spell and C. P. Christenson for discussions related to this work. 


\section{REFERENCES}

1. R. Merten, D. Lauerer, and M. Dahm, J. Cell. Plast., 4, 262(1968).

2. A. S. Tompa, Anal. Chem., 44, 1056(1972).

3. G. R. Rossmy, H. J. Kollmeier, W. Lidy, H. Schator and M. Wiemann, J. Cell. Plast., 13, 26(1977).

4. G. Menges, H. Schwesig, and G. Hahn, Org. Coat. Plast. Chem., 444, $229(1981)$.

5. F. E. Bailey, Jr. and F. E. Critchfield, J. Cell. Plast., 17, 333(1981).

5. G. Woods, Flexible Polyurethane Foams: Chemistry and Technology (Applied Science Publishers, London, 1982).

7. C. S. P. Sung, T. W. Smith, and N. H. Sung, Macromolecules, 13,117(1980).

8. C. S. P. Sung, and N. S. Schneider, Macromolecules, 8, 68(1975).

9. N. S. Schneider and C. S. P. Sung, Polym. Eng. and SCi., 17, 73(1977).

10. C. P. Christenson and R. E. Guerra, private communications. 
TABLE I. FORMULATIONS OF THE VARIOUS FOAMS USED IN THE STUDY

\begin{tabular}{|c|c|c|c|c|c|c|}
\hline Component & $31135-33-1$ & $31135-33-2$ & $31135-33-3$ & $31135-33-4$ & $31135-33-5$ & $31135-33-1$ \\
\hline VORANOL * 3100 & 100 & 100 & 100 & 100 & 200 & 100 \\
\hline Water & 2.0 & 3.0 & 4.0 & 5.0 & 4.0 & 2.0 \\
\hline $\begin{array}{r}\text { Silicone } \\
B F-2370\end{array}$ & 1.0 & 1.0 & 1.0 & 1.0 & 1.0 & 1.0 \\
\hline $\begin{array}{l}\text { Catalyst } \\
\text { Dabco 33LV }\end{array}$ & 0.3 & 0.3 & 0.3 & 0.3 & 0.3 & 0.3 \\
\hline $\operatorname{Tin}_{T-9}$ Catalyst & 0.15 & 0.15 & 0.15 & 0.15 & 0.175 & 0.10 \\
\hline $\begin{array}{l}\text { Isocyanate } \\
\text { T- } 80\end{array}$ & 30.79 & 41.43 & 52.06 & 62.70 & 52.06 & 30.79 \\
\hline Index & 110 & 110 & 110 & 110 & 110 & 110 \\
\hline
\end{tabular}

*A. trademark of the Dow Chemical Company

TABLE II. Possible carbonyls and their frequencies in the polyurethane(urea) foams and compression molded plaques.

Assignment of Carbongl Stretching Frequency $(\mathrm{cm}-1)$

Isocyanate

Carbodiimide 2270

Urea (hodrogen bonded)

Urea (Iree)

Urethane (hydrogen bonded) 2,4-TDI

$2150-2100$

Urethane (hdyrogen bonded) 2,6-TDI

1640

1695

1720

Urethane (free) 2,4 and 2,6-TDI

1700

Trimer

1740

Dimer

1709-1689

Allophanate

$1783-1770$

Biuret

$1751-1721(a)$

$1721-1695$

Carbamic acid

$1720-1690(a)$

$1708-1653$

1710

(a) higher frequency band more intense 
FIGURE 1. Plots of parts by weigh of $H 20$ versus various absorbance ratios involving the $2270 \mathrm{~cm}-1$ isocyanate band.

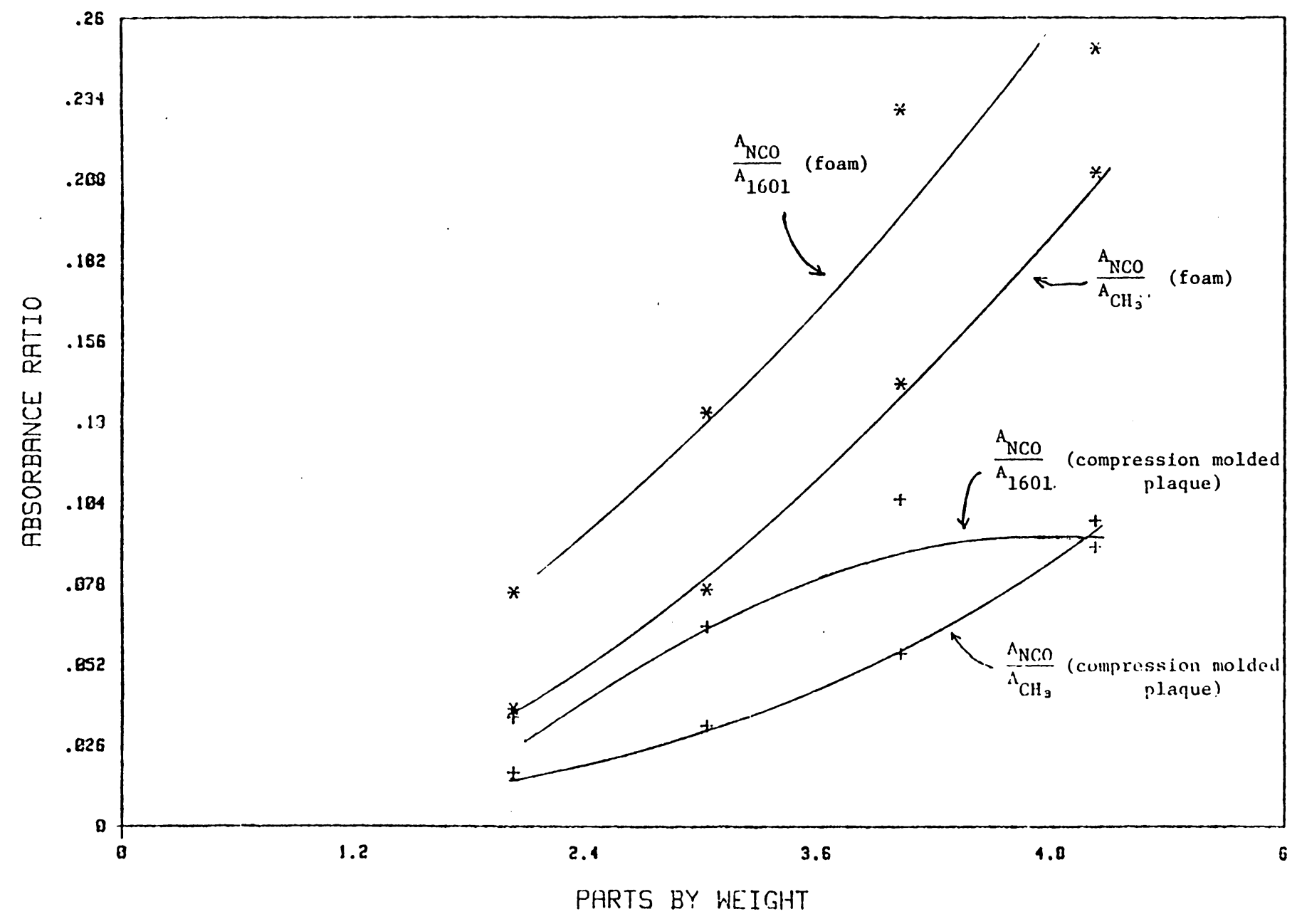




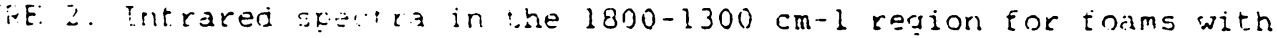 varying am unt s of water and isocyanate.}

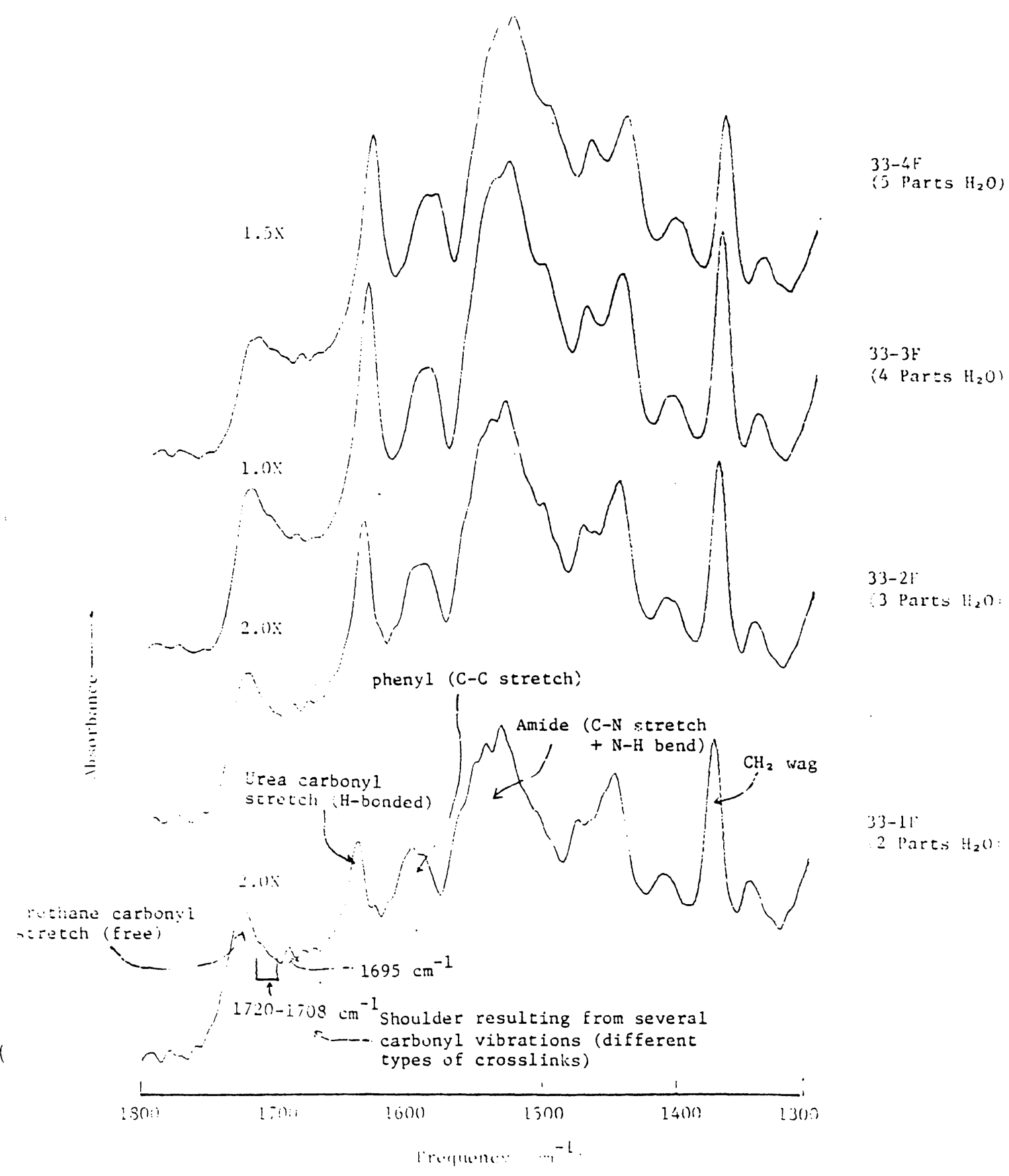


We Fis. 2 for an explanation of selected bands

.33

1

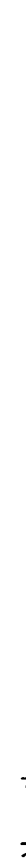

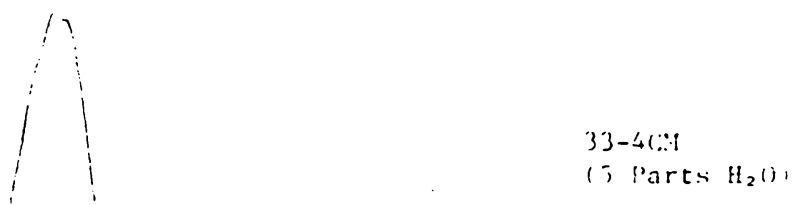
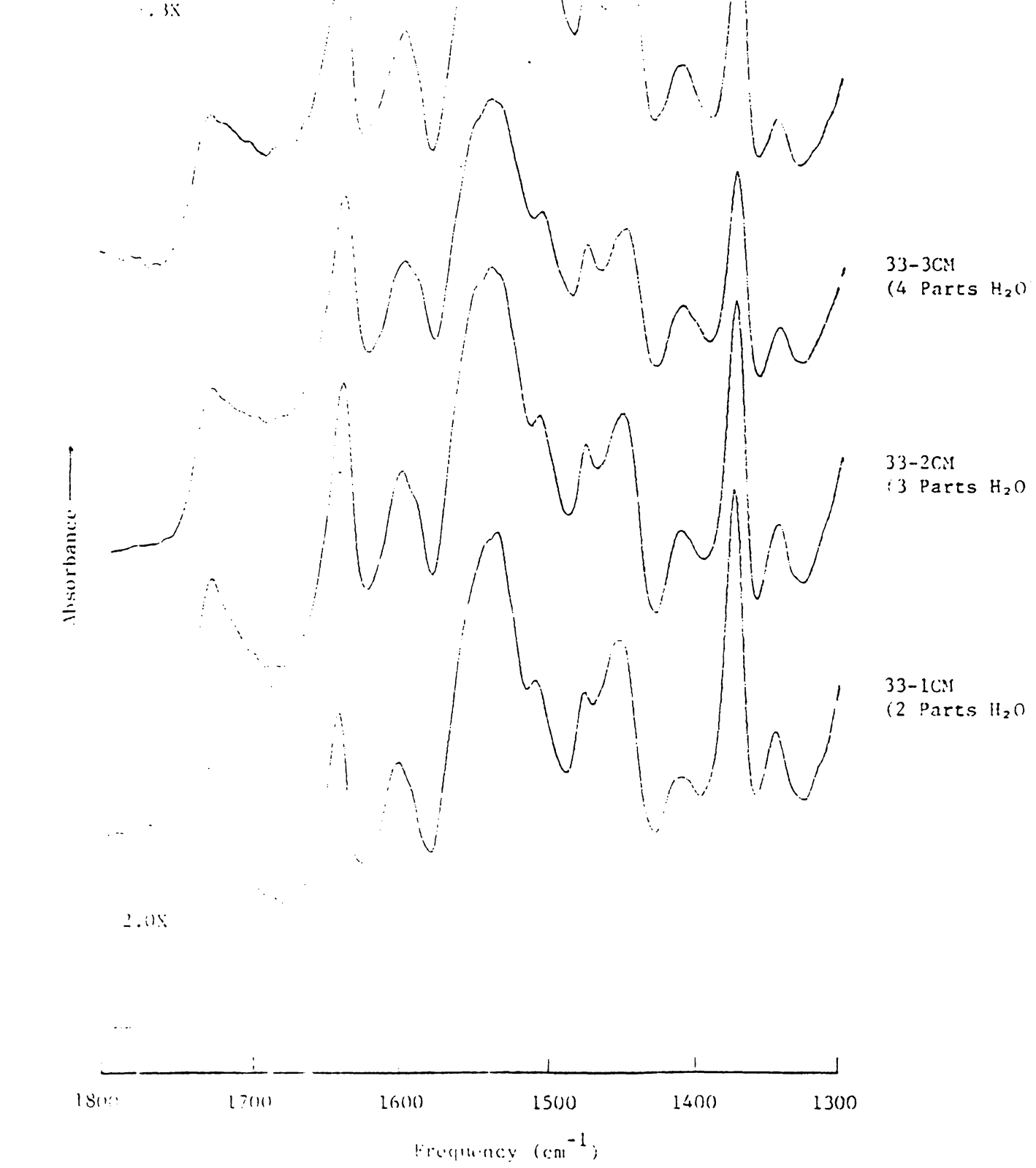

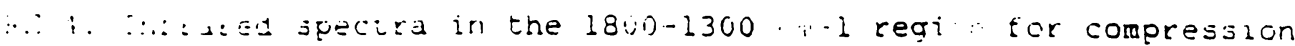
nclied plaques witk varying amount if wat ind isocyanate. 
FIGURE 3. Plot of parts by weis... of water versus various absorbance banc ratios involving the urea carbonyl $(1642 \mathrm{~cm}-1)$ and urethane carbonyl $(1732 \mathrm{~cm}-1)$ bands.

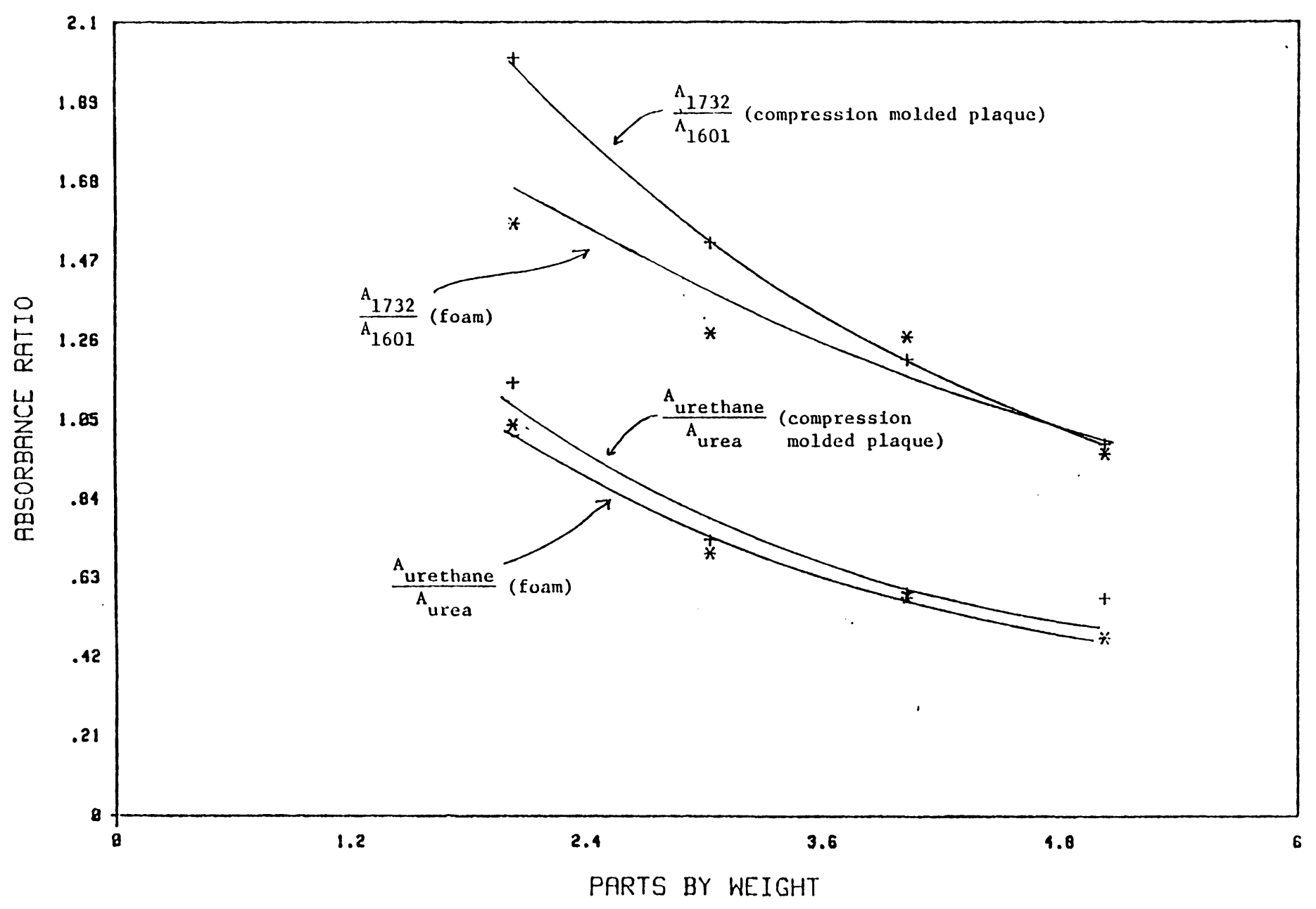



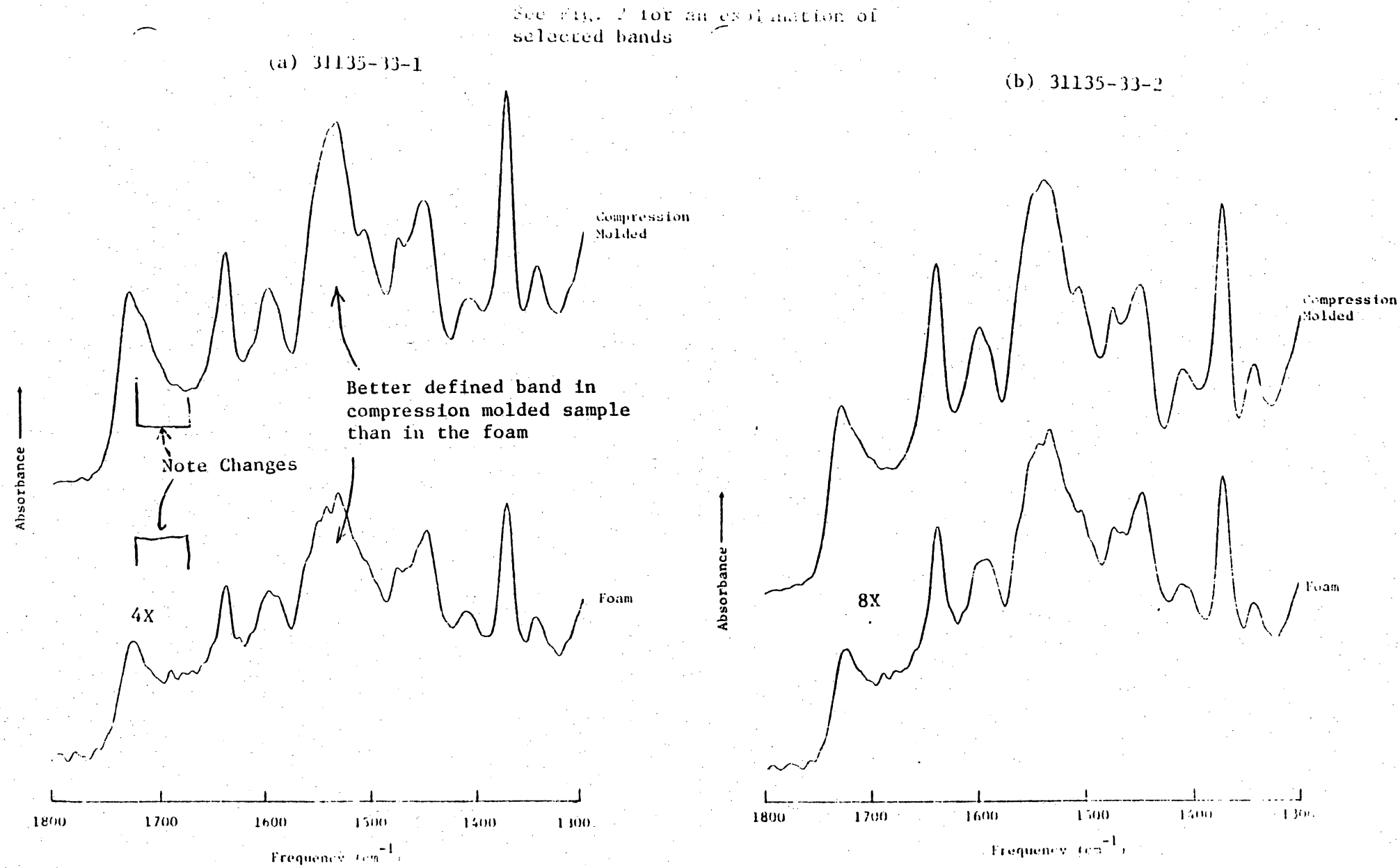

FIOHEF 5 . Comparieor of the inf rared spectra in the $1800-1300 \mathrm{rm}-1$ reation tor the lormes and ormerestion moded plaques. 
$\cdots$

(c) $31.133-33-1$

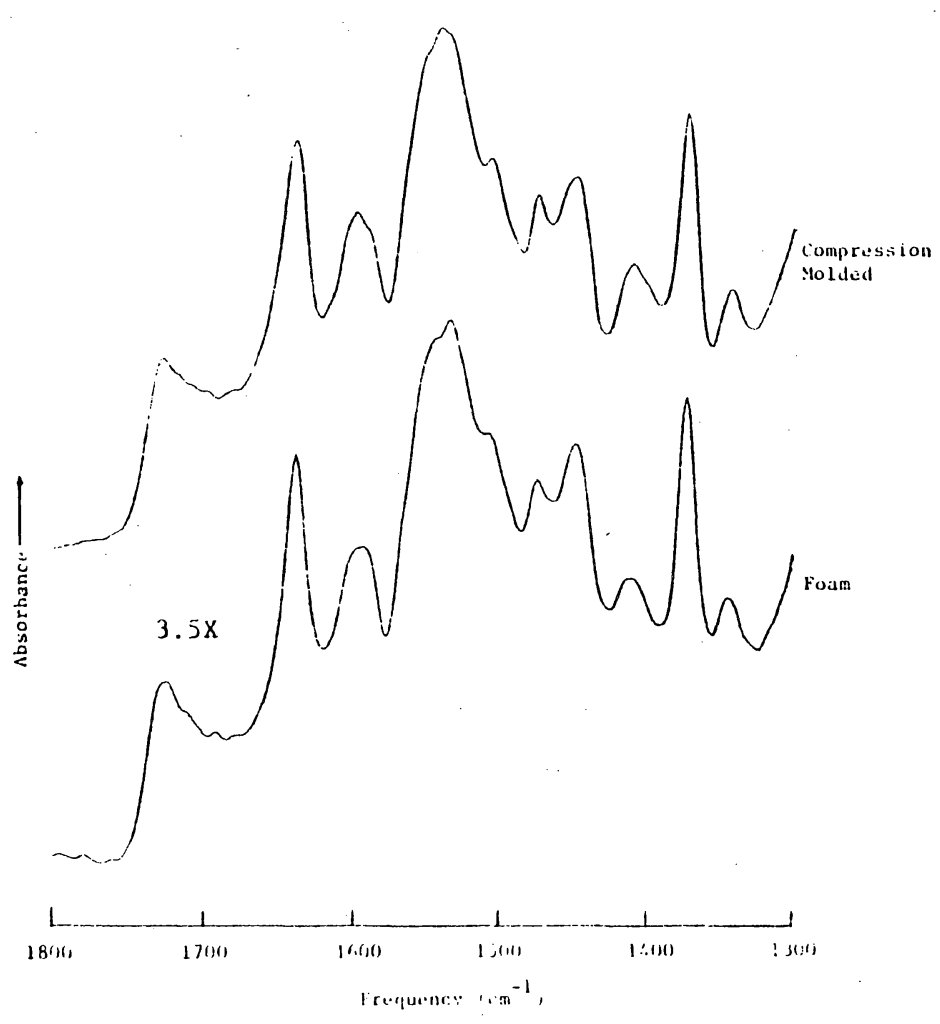

(1) $31135-33 \ldots$

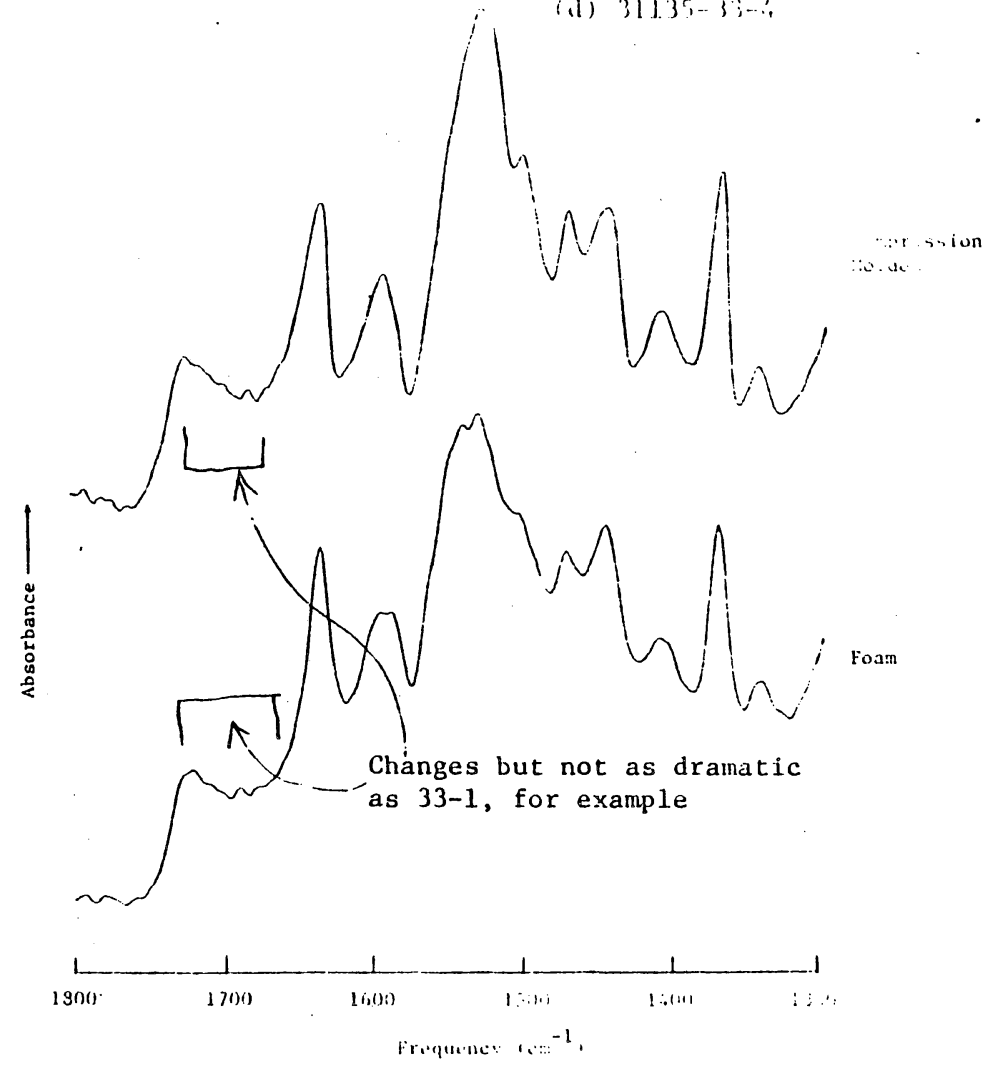

$\stackrel{N}{w}$

Fig. $5 \operatorname{con}^{1} \mathrm{t}$ 

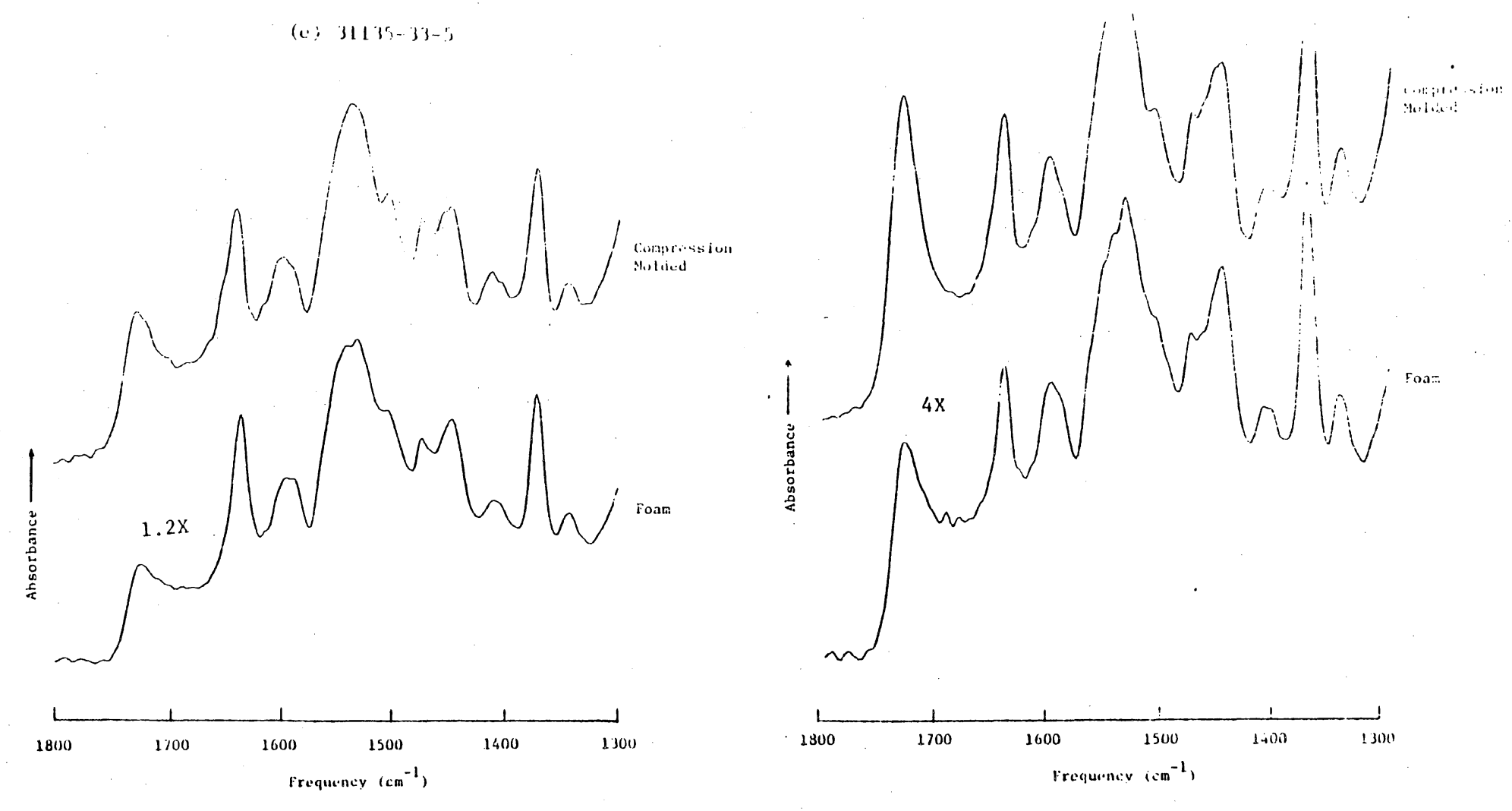

Fig. $5 \operatorname{con}^{\prime} t$ 

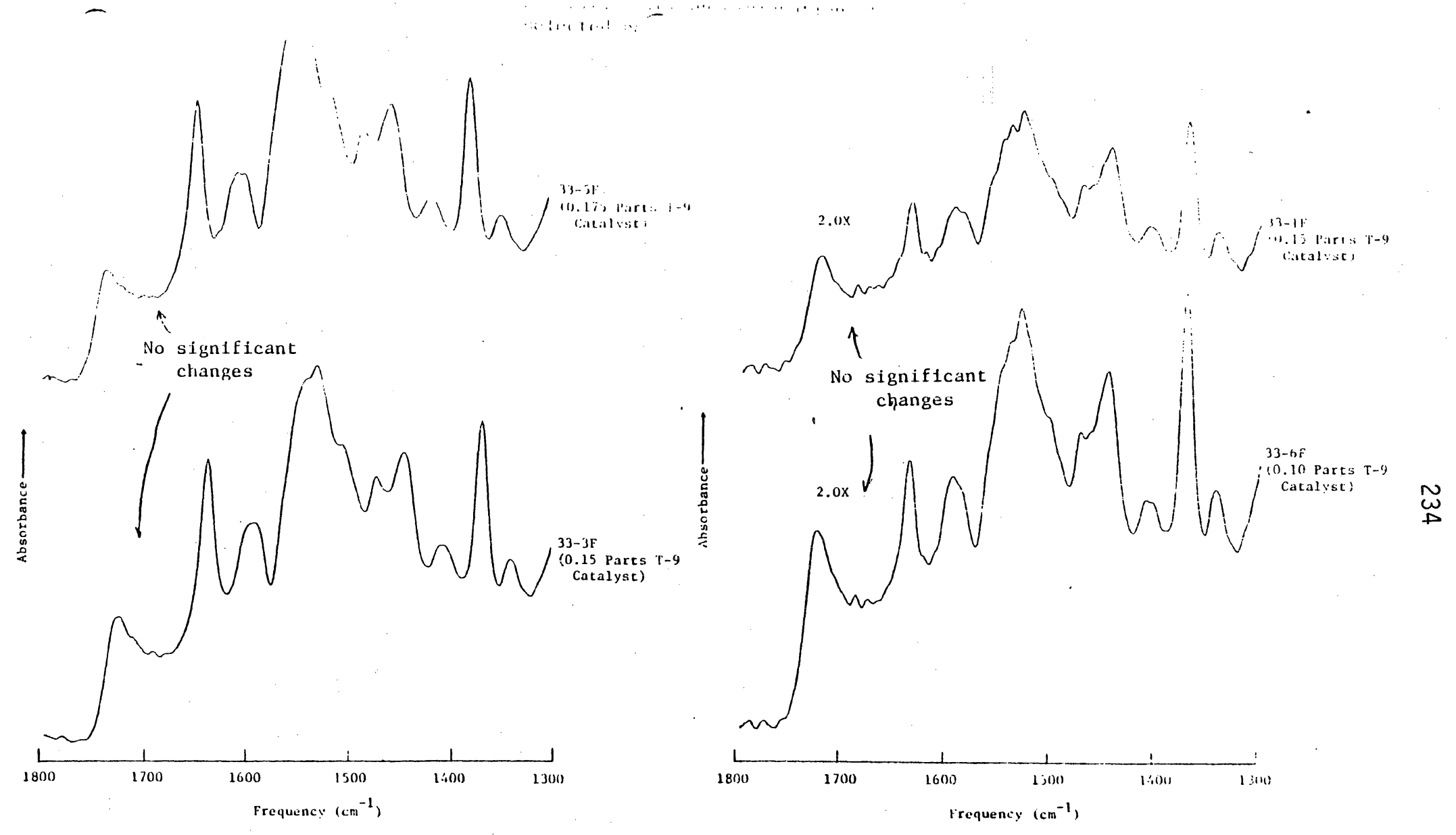

FIGURE 6. Infrared spectra in the $1800-1300 \mathrm{~cm}-1$ region for foams varyina the tin catalyst $(T-9)$ concentration. 


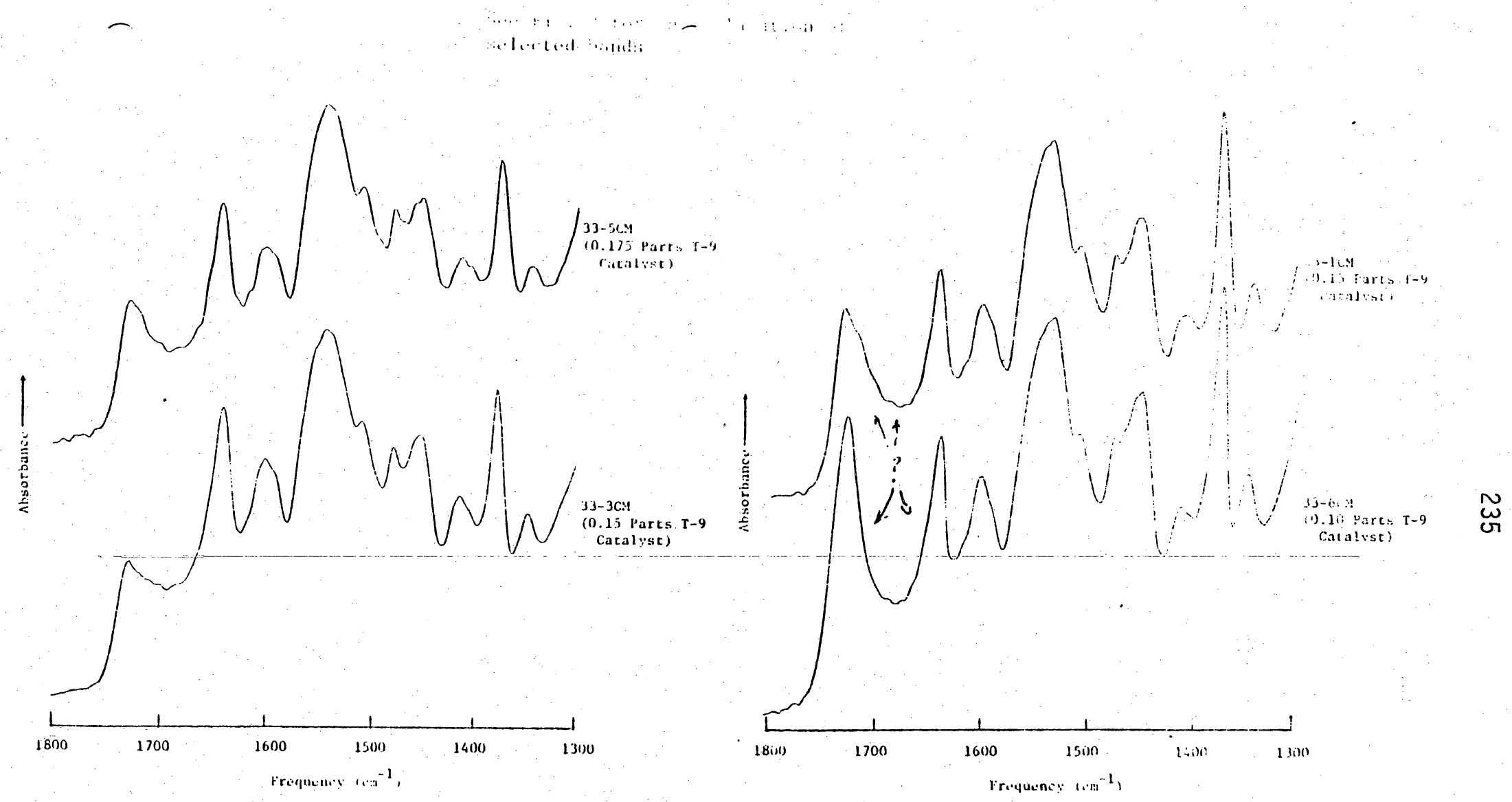

FIGURE 7. Infrared spectra in the 1800-1300 cm-1 region for compression molded plaques varying the tin catalyst (T-9) concentration. 


\section{The vita has been removed from the scanned document}

